\title{
Antibiotics and Urinary Tract Infections
}

\author{
Edited by \\ Truls E. Bjerklund Johansen and Kurt G. Naber \\ Printed Edition of the Special Issue Published in Antibiotics
}

MDPI www.mdpi.com/journal/antibiotics 
Truls E. Bjerklund Johansen and Kurt G. Naber (Eds.)

\section{Antibiotics and Urinary Tract Infections}


This book is a reprint of the special issue that appeared in the online open access journal Antibiotics (ISSN 2079-6382) in 2014 (available at:

http://www.mdpi.com/journal/antibiotics/special_issues/urinary-tract-infections).

\section{Guest Editors}

Truls E. Bjerklund Johansen

Department of Urology, Oslo University Hospital

Oslo, Norway

Kurt G. Naber

Technical University of Munich

Munich, Germany

\section{Editorial Office}

MDPI AG

Klybeckstrasse 64

Basel, Switzerland

\section{Publisher}

Shu-Kun Lin

Production Editor

Martyn Rittman

\section{Edition 2015}

MDPI • Basel • Beijing • Wuhan

ISBN 978-3-906980-80-5

(C) 2015 by the authors; licensee MDPI, Basel, Switzerland. All articles in this volume are Open Access distributed under the Creative Commons Attribution 3.0 license (http://creativecommons.org/licenses/by/3.0/), which allows users to download, copy and build upon published articles even for commercial purposes, as long as the author and publisher are properly credited, which ensures maximum dissemination and a wider impact of our publications. However, the dissemination and distribution of copies of this book as a whole is restricted to MDPI, Basel, Switzerland. 


\section{Table of Contents}

List of Contributors

\section{Editorial}

Truls E. Bjerklund Johansen and Kurt G. Naber

Urinary Tract Infections

Antibiotics 2014, 3(3), 375-377; doi:10.3390/antibiotics3030375 VIII

http:/www.mdpi.com/2079-6382/3/3/375

\section{Chapter 1}

Lilian M. Abbo and Thomas M. Hooton

Antimicrobial Stewardship and Urinary Tract Infections

Antibiotics 2014, 3(2), 174-192; doi:10.3390/antibiotics3020174

http://www.mdpi.com/2079-6382/3/2/174

\section{Chapter 2}

Daria Nicolosi, Gianna Tempera, Carlo Genovese and Pio M. Furneri

Anti-Adhesion Activity of A2-type Proanthocyanidins (a Cranberry Major Component) on

Uropathogenic E. coli and P. mirabilis Strains

Antibiotics 2014, 3(2), 143-154; doi:10.3390/antibiotics3020143

http://www.mdpi.com/2079-6382/3/2/143

\section{Chapter 3}

\section{Bernd Wiedemann, Anke Heisig and Peter Heisig}

Uncomplicated Urinary Tract Infections and Antibiotic Resistance-Epidemiological and Mechanistic Aspects

Antibiotics 2014, 3(3), 341-352; doi:10.3390/antibiotics3030341

http://www.mdpi.com/2079-6382/3/3/341

\section{Chapter 4}

Marcelo Hisano, Homero Bruschini, Antonio Carlos Nicodemo and Miguel Srougi

Uncomplicated Urinary Tract Infections in Women in a Sao Paulo Quaternary Care Hospital:

Bacterial Spectrum and Susceptibility Patterns

Antibiotics 2014, 3(1), 98-108; doi:10.3390/antibiotics3010098

http://www.mdpi.com/2079-6382/3/1/98

\section{Chapter 5}

Winifred Owumi, Niaz Banaei and Linda D. Shortliffe

Adult and Pediatric Intra-Institutional Trends of Ciprofloxacin Susceptibility in E. coli

Positive Urinary Cultures

Antibiotics 2014, 3(2), 163-173; doi:10.3390/antibiotics3020163.

http://www.mdpi.com/2079-6382/3/2/163

\section{Chapter 6}

Ceire Costelloe, O. Martin Williams, Alan A. Montgomery, Colin Dayan and

Alastair D. Hay

Antibiotic Prescribing in Primary Care and Antimicrobial Resistance in Patients Admitted to Hospital with Urinary Tract Infection: A Controlled Observational Pilot Study 


\section{Chapter 7}

Franck Bruyère, Joseph-Alain Ruimy, Louis Bernard, Raphael Elfassi, Olivier Boyer, Fabrice Amann and Paul Meria

Value of Provoked or Spontaneous Flank Pain in Men with Febrile Urinary Tract Infections Antibiotics 2014, 3(2), 155-162; doi:10.3390/antibiotics3020155.

http://www.mdpi.com/2079-6382/3/2/155

\section{Chapter 8}

Satoshi Takahashi, Hiroshi Kiyota, Shin Ito, Akihiko Iwasawa, Yoshiki Hiyama, Teruhisa Uehara, Koji Ichihara, Jiro Hashimoto, Naoya Masumori, Kenichi Sunaoshi, Koichi Takeda, Nobukazu Suzuki, Takahide Hosobe, Hirokazu Goto, Hidenori Suzuki and Shoichi Onodera

Clinical Efficacy of a Single Two Gram Dose of Azithromycin Extended Release for Male Patients with Urethritis

Antibiotics 2014, 3(2), 109-120; doi:10.3390/antibiotics3020109

http://www.mdpi.com/2079-6382/3/2/109

\section{Chapter 9}

Joey Lo, Dirk Lange and Ben H. Chew

Ureteral Stents and Foley Catheters-Associated Urinary Tract Infections: The Role of Coatings and Materials in Infection Prevention

Antibiotics 2014, 3(1), 87-97; doi:10.3390/antibiotics3010087

http://www.mdpi.com/2079-6382/3/1/87

\section{Chapter 10}

Walid Elkhatib and Ayman Noreddin

In Vitro Antibiofilm Efficacies of Different Antibiotic Combinations with Zinc Sulfate against Pseudomonas aeruginosa Recovered from Hospitalized Patients with Urinary Tract Infection Antibiotics 2014, 3(1), 64-84; doi:10.3390/antibiotics3010064

http://www.mdpi.com/2079-6382/3/1/64

\section{Chapter 11}

\section{Maria Jose Munoz-Davila}

Role of Old Antibiotics in the Era of Antibiotic Resistance. Highlighted Nitrofurantoin for the Treatment of Lower Urinary Tract Infections Antibiotics 2014, 3(1), 39-48; doi:10.3390/antibiotics3010039

http://www.mdpi.com/2079-6382/3/1/39

\section{Chapter 12}

Ekaterina Kulchavenya, Irina Felker and Elena Brizhatyuk

International Conference "Urogenital Infections and Tuberculosis" in Novosibirsk, Russia, Has Opened New Perspectives in the Fight against Tuberculosis Antibiotics 2014, 3(2), 121-127; doi:10.3390/antibiotics3020121 


\section{List of Contributors}

Fabrice Amann: General Practitioner, 15 rue Jean Baptiste Berlier, Paris 75013, France

Niaz Banaei: Departments of Pathology and Medicine, Stanford University Medical Center, 300 Pasteur Drive, Stanford, CA 94304, USA

Louis Bernard: University Francois Rabelais de Tours, PRES Centre Val de Loire Université, Tours 37000, France; Infectious Disease Department, University Hospital of Tours, Loire Valley 37044, France

Olivier Boyer: General Practitioner, 15 rue Jean Baptiste Berlier, Paris 75013, France

Elena Brizhatyuk: Novosibirsk Research Institute for Tuberculosis, Novosibirsk 630040, Russia

Homero Bruschini: Division of Urology, Hospital das Clinicas-SP, University of Sao Paulo, 255

Doutor Enéas Carvalho de Aguiar Ave., 7th floor, São Paulo 05403-000, Brazil

Franck Bruyère: Urology Department, University Hospital of Tours, Loire Valley 37044, France; University Francois Rabelais de Tours, PRES Centre Val de Loire Université, Tours 37000, France

Ben H. Chew: Department of Urologic Sciences, University of British Columbia, 2660 Oak Street, Vancouver, BC, V6H 3Z6, Canada

Ceire Costelloe: Centre for Primary Care and Public health, Blizzard Institute, Queen Mary University of London, London E1 2AB, UK

Colin Dayan: Institute of Molecular \& Experimental Medicine, Cardiff University School of Medicine, Wales Heart Research Institute, Heath Park, Cardiff CF14 4XN, UK

Raphael Elfassi: General Practitioner, 15 rue Jean Baptiste Berlier, Paris 75013, France

Walid Elkhatib: Department of Microbiology and Immunology, Faculty of Pharmacy, Ain Shams University, African Union Organization St. Abbassia, Cairo 11566, Egypt; Department of Pharmacy Practice, School of Pharmacy, Hampton University, Kittrell Hall Hampton, Virginia 23668, USA

Irina Felker: Novosibirsk Research Institute for Tuberculosis, Novosibirsk 630040, Russia

Pio M. Furneri: Department of Biomedical Sciences, Section of Microbiology, University of Catania, Via Androne 81, Catania 95124, Italy

Carlo Genovese: Department of Biomedical Sciences, Section of Microbiology, University of Catania, Via Androne 81, Catania 95124, Italy

Hirokazu Goto: Department of Urology, Fuji City General Hospital, 50 Takashima-Cho, Fuji, Shizuoka 4178567, Japan 
Jiro Hashimoto: Department of Urology, Sapporo Medical University School of Medicine, S. 1, W. 16, Chuo-ku, Sapporo, Hokkaido 0608543, Japan

Alastair D. Hay: Centre for Academic Primary Care, NIHR School for Primary Care Research, School of Social and Community Medicine, University of Bristol, Canynge Hall, Bristol BS8 2PS, UK

Anke Heisig: Pharmaceutical Biology and Microbiology, Institute of Biochemistry and Molecular Biology, University of Hamburg, Bundesstrasse 45, 20146 Hamburg, Germany

Peter Heisig: Pharmaceutical Biology and Microbiology, Institute of Biochemistry and Molecular Biology, University of Hamburg, Bundesstrasse 45, 20146 Hamburg, Germany

Marcelo Hisano: Division of Urology, Hospital das Clinicas-SP, University of Sao Paulo, 255 Doutor Enéas Carvalho de Aguiar Ave., 7th floor, São Paulo 05403-000, Brazil

Yoshiki Hiyama: Department of Urology, Sapporo Medical University School of Medicine, S. 1, W. 16, Chuo-ku, Sapporo, Hokkaido 0608543, Japan

Takahide Hosobe: Hosobe Clinic, 1-1-15, Nezu, Bunkyo-ku, Tokyo 1130031, Japan

Koji Ichihara: Department of Urology, Sapporo Medical University School of Medicine, S. 1, W. 16, Chuo-ku, Sapporo, Hokkaido 0608543, Japan

Shin Ito: iClinic, 5-9-6, Nagamachi, Taihaku-ku, Sendai, Miyagi 9820011, Japan

Akihiko Iwasawa: Iwasawa Clinic, Sapporo Medical University School of Medicine, S. 1, W. 16, Chuo-ku, Sapporo, Hokkaido 0600061, Japan

Truls E. Bjerklund Johansen: Urology Department, Oslo University Hospital, 0424 Oslo, Norway

Hiroshi Kiyota: Department of Urology, the Jikei University Katsushika Medical Center, 6-41-2 Aoto Katsushika-ku, Tokyo 1258506, Japan

Ekaterina Kulchavenya: Novosibirsk Research Institute for Tuberculosis, Novosibirsk 630040, Russia; Novosibirsk State Medical University, Novosibirsk 630040, Russia

Dirk Lange: Department of Urologic Sciences, University of British Columbia, 2660 Oak Street, Vancouver, BC, V6H 3Z6, Canada

Joey Lo: Department of Urologic Sciences, University of British Columbia, 2660 Oak Street, Vancouver, BC, V6H 3Z6, Canada

Naoya Masumori: Department of Urology, Sapporo Medical University School of Medicine, S. 1, W. 16, Chuo-ku, Sapporo, Hokkaido 0608543, Japan

Paul Meria: Urology Department, Saint Louis Hospital, Paris 75010, France

Alan A. Montgomery: Faculty of Medicine and Clinical Sciences, University of Nottingham, Nottingham NG7 2UH, UK 
Maria Jose Munoz-Davila: Microbiology laboratory, Rafael Méndez Hospital, Ctra. Nacional 340, Km. 589, Lorca, Murcia 30817, Spain

Kurt G. Naber: Urology Department, Technical University of Munich, Munich 81675, Germany Antonio Carlos Nicodemo: Infectious Disease Department, Hospital das Clinicas-SP, University of Sao Paulo, 255 Doutor Enéas Carvalho de Aguiar Ave., 4th floor, São Paulo 05403-000, Brazil

Daria Nicolosi: Department of Biomedical Sciences, Section of Microbiology, University of Catania, Via Androne 81, Catania 95124, Italy

Ayman Noreddin: Department of Pharmacy Practice, School of Pharmacy, Hampton University, Kittrell Hall Hampton, Virginia 23668, USA; Graduate Program of Biomedical Sciences, Eastern Virginia Medical School, 825 Fairfax Ave, Norfolk, Virginia 23507, USA

Shoichi Onodera: Department of Urology, Fuji City General Hospital, 50 Takashima-Cho, Fuji, Shizuoka 4178567, Japan

Winifred Owumi: Department of Urology, Stanford University Medical Center, 750 Welch Rd, MC: 5725, Stanford, CA 94305-5725, USA

Joseph-Alain Ruimy: General Practitioner, 15 rue Jean Baptiste Berlier, Paris 75013, France

Linda D. Shortliffe: Department of Urology, Stanford University Medical Center, 750 Welch Rd, MC: 5725, Stanford, CA 94305-5725, USA

Miguel Srougi: Division of Urology, Hospital das Clinicas-SP, University of Sao Paulo, 255 Doutor Enéas Carvalho de Aguiar Ave., 7th floor, São Paulo 05403-000, Brazil

Kenichi Sunaoshi: Teine Urologic Clinic, 1-12, Maeda 6-7, Teine-ku, Sapporo 0060816, Japan

Hidenori Suzuki: Department of Urology, Fuji City General Hospital, 50 Takashima-Cho, Fuji, Shizuoka 4178567, Japan

Nobukazu Suzuki: Teine Urologic Clinic, 1-12, Maeda 6-7, Teine-ku, Sapporo 0060816, Japan

Satoshi Takahashi: Department of Urology, Sapporo Medical University School of Medicine, S.

1, W. 16, Chuo-ku, Sapporo, Hokkaido 0608543, Japan

Koichi Takeda: Teine Urologic Clinic, 1-12, Maeda 6-7, Teine-ku, Sapporo 0060816, Japan

Gianna Tempera: Department of Biomedical Sciences, Section of Microbiology, University of Catania, Via Androne 81, Catania 95124, Italy

Teruhisa Uehara: Department of Urology, Sapporo Medical University School of Medicine, S. 1, W. 16, Chuo-ku, Sapporo, Hokkaido 0608543, Japan

Bernd Wiedemann: Böstens Hoi 15, 24882 Schaalby, Germany

Owen M Williams: Health Protection Agency Microbiology Services, United Hospital Bristol Trust, Bristol Royal Infirmary, Marlborough Street, Bristol BS1 3NU, UK 


\title{
Editorial
}

\section{Urinary Tract Infections}

\author{
Truls E. Bjerklund Johansen and Kurt G. Naber
}

Reprinted from Antibiotics. Cite as: Johansen, T.E.B.; Naber, K.G. Urinary Tract Infections. Antibiotics 2014, 3, 375-377.

Urinary tract infections (UTI) are among the most frequently acquired infections in the community, but also in hospitals and other health care institutions, causing a huge amount of antibiotic consumption. During the last decade we have seen significant changes in the field of urinary tract infections regarding causative pathogens and antibiotic treatment calling for an update of current trends.

The worldwide increase of uropathogens resistant to former first line antibiotics, such as cotrimoxazole, fluoroquinolones and cephalosporins, has had detrimental consequences not only for treatment but also for prophylaxis of infectious complications after urological interventions. A paradigm shift concerning asymptomatic bacteriuria has had a great impact on the definition and management of UTIs today [1-4].

For uncomplicated lower UTI, such as acute cystitis in otherwise healthy women, not only a revival of old (oral) antibiotics, such as fosfomycin trometamol, pivmecillinam, nitrofurantoin, can be observed in many guidelines [5-7], but even a non-antimicrobial measure has been tested in a pilot study [8]. It will therefore be interesting to see the results of forthcoming phase III studies and whether antibiotic therapy could at least be partially replaced. For prophylaxis of recurrent episodes of uncomplicated UTI, non-antimicrobial measures are already preferred and antimicrobial prophylaxis is only recommended as a last resort [7].

However, for complicated, nosocomial and severe UTI including pyelonephritis, antibiotic therapy will still be a corner stone in combination with treatment of the underlying complicating conditions. Unfortunately, there are few new antimicrobial drugs in the pipelines of pharmaceutical companies with prospects to overcome the problem of multi and extended drug resistant uropathogens [9].

Although the classical distinction between uncomplicated and complicated UTI is still valid in principle, the different criteria to be considered are so heterogeneous, that a better (phenotypical) subclassification might be helpful, as proposed by the European Section of Infection of Urology (ESIU) of the European Association of Urology (EAU) [10].

In consideration of so many new aspects related to optimal management of UTI, it has been our pleasure to edit a joint presentation of the results from different research groups in one special scientific publication challenging established as well as new scientific approaches to improve prophylaxis and treatment of patients suffering from UTI. 


\section{Conflicts of Interest}

The authors declare no conflict of interest.

\section{References}

1. Naber, K.G.; Schito, G.; Botto, H.; Palou, J.; Mazzei, T. Surveillance study in Europe and Brazil on clinical aspects and antimicrobial resistance epidemiology in females with cystitis (ARESC): Implications for empiric therapy. Eur. Urol. 2008, 54, 1164-1178.

2. Tandogdu, Z.; Cek, M.; Wagenlehner, F.; Naber, K.; Tenke, P.; van Ostrum, E.; Bjerklund Johansen, T. Resistance patterns of nosocomial urinary tract infections in urology departments: 8-Year results of the global prevalence of infections in urology study. World J. Urol. 2014, 32, 791-801.

3. Wagenlehner, F.M.E.; van Oostrum, E.; Tenke, P.; Tandogdu, Z.; Cek, M.; Grabe, M.; Wullt, B.; Pickard, R.; Naber, K.G.; Pilatz, A.; et al. Infective complications after prostate biopsy: Outcome of the Global Prevalence Study of Infections in Urology (GPIU) 2010 and 2011. A prospective multinational multicentre prostate biopsy study. Eur. Urol. 2013, 63, 521-527.

4. Wagenlehner, F.M.E.; Naber, K.G. Asymptomatic bacteriuria-Shift of paradigm. Clin. Infect. Dis. 2012, 55, 778-780.

5. Gupta, K.; Hooton, T.M.; Naber, K.G.; Wullt, B.; Colgan, R.; Miller, L.G.; Moran, G.J.; Nicolle, L.E.; Raz, R.; Schaeffer, A.J.; et al. International clinical practice guidelines for the treatment of acute uncomplicated cystitis and pyelonephritis in women: A 2010 update by the Infectious Diseases Society of America and the European Society for Microbiology and Infectious Diseases. Clin. Infect. Dis. 2011, 52, e103-e120.

6. Wagenlehner, F.M.E.; Hoyme, U.; Kaase, M.; Fünfstück, R.; Naber, K.G.; Schmiemann, G. Clinical practice guidelines: Uncomplicated urinary tract infections. Dtsch. Arztebl. Int. 2011, 108, 415-423.

7. Grabe, M.; Bjerklund-Johansen, T.E.; Bartoletti, R.; Çek, M.; Naber, K.G.; Pickard, R.S.; Tenke, P.; Wagenlehner, F.; Wullt, P. Guidelines on urological infections. European Association of Urology 2014. Available online: http://www.uroweb.org/gls/pdf/19\%20Urological\% 20infections_LR.pdf(accessed on 13 July 2014).

8. Bleidorn, J.; Gagyor, I.; Kochen, M.M.; Wegscheider, K.; Hummers-Pradier, E. Symptomatic treatment (ibuprofen) or antibiotics (ciprofloxacin) for uncomplicated urinary tract infection?-Results of a randomized controlled pilot trial. BMC Med. 2010, 8, e30.

9. Magiorakos, A.P.; Srinivasan, A.; Carey, B.; Carmeli, Y.; Falagas, M.E.; Giske, C.G.; Harbarth, S.; Hindler, J.F.; Kahlmeter, G.; Olsson-Liljequist, B.; et al. Multidrug-resistant, extensively drug-resistant and pandrug-resistant bacteria: An international expert proposal for interim standard definitions for acquired resistance. Clin. Microbiol. Infect. 2012, 18, 268-281.

10. Bjerklund Johansen, T.E.; Botto, H.; Cek, M.; Grabe, M.; Tenke, P.; Wagenlehner, F.M.E.; Naber, K.G. Critical review of current definitions of urinary tract infections and proposal of an EAU/ESIU classification system. Int. J. Antimicr. Agents 2011, 38S, 64-70. 


\section{Chapter 1}

\section{Antimicrobial Stewardship and Urinary Tract Infections}

\section{Lilian M. Abbo and Thomas M. Hooton}

Abstract: Urinary tract infections are the most common bacterial infections encountered in ambulatory and long-term care settings in the United States. Urine samples are the largest single category of specimens received by most microbiology laboratories and many such cultures are collected from patients who have no or questionable urinary symptoms. Unfortunately, antimicrobials are often prescribed inappropriately in such patients. Antimicrobial use, whether appropriate or inappropriate, is associated with the selection for antimicrobial-resistant organisms colonizing or infecting the urinary tract. Infections caused by antimicrobial-resistant organisms are associated with higher rates of treatment failures, prolonged hospitalizations, increased costs and mortality. Antimicrobial stewardship consists of avoidance of antimicrobials when appropriate and, when antimicrobials are indicated, use of strategies to optimize the selection, dosing, route of administration, duration and timing of antimicrobial therapy to maximize clinical cure while limiting the unintended consequences of antimicrobial use, including toxicity and selection of resistant microorganisms. This article reviews successful antimicrobial stewardship strategies in the diagnosis and treatment of urinary tract infections.

Reprinted from Antibiotics. Cite as: Abbo, L.M.; Hooton, T.M. Antimicrobial Stewardship and Urinary Tract Infections. Antibiotics 2014, 3, 174-192.

\section{Introduction}

Urinary tract infections (UTIs) are the most common bacterial infection encountered in ambulatory care settings in the United States, accounting for 8.6 million visits in 2007 [1,2]. Likewise, catheter-associated UTIs are the most common type of healthcare-associated infection reported to the National Healthcare Safety Network (NHSN) [3] and the most commonly treated infections in residents of long-term care facilities (LTCF) each year [4]. In a recent study by Sammon et al. [5], 10.8 million patients in the United States visited an Emergency Department (ED) for the treatment of a UTI between 2006 and 2009. The economic burden of utilizing the ED for the treatment of UTIs is estimated to be $\$ 2$ billion US dollars annually, with mean charges being 10 times higher for patients who were treated and released from EDs (\$2000 per visit) compared with treatment in an outpatient clinic (\$200) [5].

Starting or reassessing antimicrobial prescriptions based on clinical context, symptomatology and susceptibility data are of paramount importance in all clinical situations and particularly when dealing with UTIs [6]. Urine samples are the largest single category of specimens received by most microbiology laboratories, and the majority of urine cultures do not yield clinically significant results [7]. The diagnosis of UTI is primarily based on signs and symptoms rather than isolated 
laboratory findings; importantly, bacteriuria is not a disease [8]. Thus, the collection and interpretation of urine cultures should be based on the clinical scenario. Cultures are not recommended for most women with acute uncomplicated cystitis because the microbiology and therapeutic approach for these women is consistent, and short course therapy is effective. However, for individuals with acute pyelonephritis or complicated UTI it is important to obtain a urine culture prior to empiric therapy in order to appropriately tailor the antimicrobial regimen if necessary. In patients with indwelling urinary catheters and residents of long term care facilities, populations with high prevalences of bacteriuria, decisions as to whether to obtain a urine culture and treat should be made carefully in order to avoid inappropriate antimicrobial treatment of bacteriuria that is not associated with symptoms.

Clinicians are frequently faced with the risk-assessment decision to balance the short- and long-term risks and benefits of prescribing an antimicrobial. The short-term risks for the individual prescriber and patient include failure to treat a blossoming symptomatic infection with potential clinical worsening of the patient. The long-term benefits of not prescribing antimicrobials in asymptomatic patients, such as avoiding the emergence of antimicrobial-resistant organisms and adverse events, including Clostridium difficile infection, are less tangible to the prescriber focused on the individual rather than the ecological effects with impact at the population level [9].

Antimicrobial resistance is a major public health problem worldwide, caused in part by the overuse of antimicrobials in clinical situations where they are not necessary or in prolonged courses of therapy when shorter durations are as effective [10-12]. Antimicrobial prescribing should be prudent, thoughtful and rational. The choice of antimicrobial agents should be individualized based on the patient's allergy history, local practice patterns, prevalence of resistance, availability, cost and compliance [13]. Unfortunately, in many parts of the world fluoroquinolones are the most commonly prescribed antimicrobials for uncomplicated UTIs even though narrower spectrum cost-effective alternatives are available; their use should be minimized considering their adverse ecologic effects [13]. Several studies in adults and children have demonstrated that short-term antimicrobial courses are as effective as longer ones for the treatment of uncomplicated UTIs and many complicated UTIs [14-16], although there still remain many questions as to the optimal duration of treatment for many types of complicated UTIs. It is the responsibility of all healthcare providers to practice antimicrobial stewardship and to avoid the unnecessary use of antimicrobials $[17,18]$.

\section{Definitions}

For the purpose of this review, uncomplicated UTIs include episodes of acute cystitis and pyelonephritis occurring in healthy, non-pregnant, non-immunocompromised women with no history suggestive of an abnormal anatomical or functional urinary tract and no signs of systemic infection. All other UTIs are considered complicated [2]. The classification of UTIs according to the individual host and the severity of location have therapeutic implications in antimicrobial stewardship. International treatment guidelines recommend short-course (single-dose to 3-day regimens) regimens for acute uncomplicated cystitis [13]. Moreover, a Cochrane review [19] of 15 studies, including 1644 elderly women, concluded that short courses (3-6 days) are as effective as long courses (7-14 days) for treating uncomplicated cystitis in elderly women. Short-course regimens are 
also effective tor treatment of cystitis in pregnant women, UTIs that are generally considered to be "complicated" [20]. Moreover, short-course regimens have been found to be effective for more complicated UTIs - thus, a 5-day course of levofloxacin $750 \mathrm{mg}$ daily was found to be as effective as longer courses of therapy for the treatment of acute pyelonephritis and complicated UTI [15].

Asymptomatic bacteriuria (ASB) is defined as the presence of bacteriuria in urine revealed by quantitative culture in a sample taken from a patient without symptoms suggestive of lower or

upper UTI. In women, the traditional quantitative definition for ASB is $10^{5} \mathrm{cfu} / \mathrm{mL}$ in 2 consecutive voided urine specimens and for ASB in men a voided urine specimen with 1 bacterial species isolated in a quantitative count of $10^{5} \mathrm{cfu} / \mathrm{mL}$ [8]. In general, treatment of ASB is not indicated and may be associated with adverse outcomes, including subsequent antimicrobial resistance, C. difficile infection, adverse drug effects, and increased cost. However, ASB is associated with complications in some populations, and should therefore be screened for and treated if present in pregnancy and during interventions that compromise the urinary tract mucosa [21]. Despite the fact that UTI in patients with diabetes mellitus is associated with more severe and uncommon complications, screening for and treatment of ASB in diabetics are not recommended [22].

\section{Microbiology}

E. coli causes $75 \%$ to $95 \%$ of episodes of ASB, cystitis and pyelonephritis in young healthy women, with a minority of cases caused by other Enterobacteriaceae, other Gram negative rods, Enterococcus faecalis, Staphylococcus species and Group B streptococcus. In men and women with "complicating factors", the causative uropathogens are more variable.

\section{Antimicrobial Stewardship Opportunities}

\subsection{Does the Patient Have a UTI? Are Antimicrobials Necessary?}

Antimicrobial stewardship opportunities are summarized in Table 1. In general, symptomatic UTIs should be treated with antimicrobials to alleviate symptoms and, in the case of more serious infection, to prevent complications, whereas ASB generally does not warrant treatment. Thus, the first question a clinician should ask when considering antimicrobial therapy is whether the patient is symptomatic and if such signs and symptoms are likely caused by bacteriuria. To assist clinicians in differentiating symptomatic UTIs from ASB, several reviews and consensus guidelines have been published that provide criteria for diagnosis and management of suspected uncomplicated UTIs and those occurring in acute and long term care facilities [2,8,23-26]. Cystitis is usually manifested as dysuria with or without frequency, urgency, suprapubic pain or hematuria. Clinical signs of pyelonephritis include fever (temperature $>38^{\circ} \mathrm{C}$ ), flank pain, chills, costo-vertebral angle tenderness, and nausea and vomiting [2]. In women, absence of vaginal symptoms in the setting of UTI symptoms increases the likelihood that a UTI is present [23]. It may be very difficult to determine whether symptoms are associated with bacteriuria in patients with altered sensation, such as those with spinal cord injury and neurogenic bladder [25]. 
Table 1. Opportunities for antimicrobial stewardship and urinary tract infections.

Use antimicrobials only when appropriate

- ASB should be screened for and treated only in select conditions, such as pregnancy and prior to urologic surgery

Use the appropriate antimicrobial

- Empiric choice - for cystitis, use an agent with low risk of collateral damage

- For uncomplicated pyelonephritis and complicated UTIs, obtain pre-treatment urine culture and de-escalate as appropriate to narrow spectrum agent

Treat for appropriate duration

- Use short-course treatment for cystitis

- Short-course regimens are appropriate for some patients with complicated UTI

Consider non-antimicrobial preventive strategies for recurrent uncomplicated cystitis *

- Behavioral modification

- D-mannose

- Cranberry

- Topical estrogens in postmenopausal women

- Probiotics

- Oral immunostimulants

- Antimicrobials as a last resort

* Most non-antimicrobial preventive strategies have either not been studied in prospective trials or have not been shown to be effective in trials to date, but are reasonable to try or continue if the patient so chooses and if they are considered to be safe.

Laboratory parameters aid in the diagnosis of UTI but are not helpful in isolation. Furthermore, results of voided midstream urine cultures should be interpreted with caution. In a recent study Hooton et al. [27] analyzed microbial species and colony counts in urine samples from 226 healthy women (aged 18-49 years) with symptoms of cystitis. They found that the detection of E. coli in voided midstream urine at colony counts as low as $10-10^{2} \mathrm{cfu} / \mathrm{mL}$ was highly predictive of its presence in the bladder (positive predictive values of $93 \%$ for growth of $\geq 10^{2} \mathrm{cfu} / \mathrm{mL}$ and $99 \%$ for $\geq 10^{4} \mathrm{cfu} / \mathrm{mL}$ ). On the other hand, growth of enterococcus species and Group B streptococci in voided urine was not predictive of their growth in bladder urine and suggest that these organisms are likely to be urethral contaminants instead. The usefulness of voided urine cultures in other populations has not been studied.

\subsection{Antimicrobial Selection}

Antimicrobial resistance varies over time and by patient population in different geographic locations. If antimicrobial therapy is indicated for UTI, it is important to determine the correct drug, dose and duration of therapy. Sometimes, as with acute uncomplicated cystitis, the clinical presentation is suggestive of a predominant organism (E. coli) with predictable antimicrobial susceptibility, and narrow spectrum agents are appropriate for empiric treatment. However, in other situations, as with complicated UTI, antimicrobial susceptibility is not as predictable or there may be multiple causative uropathogens, and broad spectrum agents are more appropriate. The individual risk factors, local patterns of antimicrobial resistance, presence of urinary catheters or other "complicating factors", recent or prolonged hospitalization and previous exposure to 
antimicrobials must be taken into consideration when one is considering the optimal empiric agent for treatment of UTI.

Acute uncomplicated cystitis is a benign condition, with early resolution of symptoms in $25 \%$ to $42 \%$ of women with rare progression to pyelonephritis [2]. Nonetheless, it has considerable morbidity and antimicrobials are routinely prescribed aiming for rapid symptom resolution. The Infectious Diseases Society of America (IDSA) guidelines [13] emphasize the importance of considering collateral damage (adverse effects of a drug, such as selection for resistance) when prescribing antimicrobials. They recommend four agents (nitrofurantoin, trimethoprim-sulfamethoxazole, fosfomycin, and pivmecillinam) that result in relatively little collateral damage compared with other agents. Pivmecillinam may not be available in all countries, which limits its use. Because culture results in these patients are fairly predictable, urine cultures are usually not recommended. However, cultures are recommended if there is a concern about possible antimicrobial resistance since uropathogen resistance data reflected in hospital or community antibiograms are often unreliable, due to the nature of passive surveillance, in guiding the selection of antimicrobial therapy. The IDSA treatment guidelines for uncomplicated cystitis do, however, suggest thresholds for the prevalence of resistance in the community (if reliable antibiogram data are available) above which a drug is not recommended for empiric treatment- $10 \%$ for fluoroquinolones and $20 \%$ for trimethoprim-sulfamethoxazole $[2,13]$.

In addition, pharmacokinetic properties of the antimicrobial are important depending on the site of infection. For the treatment of a complicated UTI, the drug should achieve high concentrations in urine, kidney tissue and prostate. Therefore, nitrofurantoin and fosfomycin are not recommended for upper tract infection or any complicated UTI. Fluoroquinolones have a broad spectrum of activity and penetrate tissue well and are thus the drugs of choice for empiric treatment of uncomplicated pyelonephritis and complicated UTIs. Drug resistance has made this class of antimicrobials less useful than in the past, and for patients with severe infections it is recommended that parenteral agents with more reliable activity against uropathogens be used until susceptibility data are available. Trimethoprim-sulfamethoxazole also penetrates tissue well and is an excellent agent for the treatment of uncomplicated pyelonephritis and complicated UTIs if the organism is known to be susceptible, but it should not be used empirically in such patients due to the high prevalence of resistance among uropathogens worldwide.

\subsection{Streamlining Empirical Therapy}

De-escalation or streamlining a broad-spectrum antimicrobial to a narrower spectrum agent active against the causative uropathogen once susceptibility data are available is an important antimicrobial stewardship strategy in the management of complicated infections occurring in the hospital or LTCF. In addition, selective reporting of antimicrobial susceptibilities for uropathogens is a strategy used in many microbiology laboratories to avoid the use of broad-spectrum agents and guide clinicians in the selection of antimicrobials. The impact of selective reporting on the appropriate use of antimicrobials for UTIs has been evaluated in a randomized study [6] and several prospective surveys [28,29]. Coupat et al. [6] randomly assigned residents at 3 French universities to an intervention group that received susceptibility reporting for only 2 to 4 antimicrobials for case-vignettes, or to a control group that received full-length reporting for 25 antimicrobials. Selective 
reporting improved the appropriateness of antimicrobial choices by $7 \%$ to $41 \%$, depending on the vignette. In addition, most residents in the intervention group reported that selective reporting facilitated their choice of antimicrobials. Selective susceptibility reporting has been associated with a direct effect on antimicrobial prescribing by community clinicians in the United Kingdom [29]. Tailoring antimicrobial therapy based on local guidelines and culture results and selectively reporting susceptibility for uropathogens are important stewardship practices to improve the appropriate use of antimicrobials.

\subsection{Selecting the Correct Dose and Route}

Pharmacokinetic and pharmacodynamic properties should be considered when treating a UTI in order to achieve optimal tissue levels and effectively eradicate the infection. Antimicrobials that are characterized by concentration-dependent killing (e.g., aminoglycosides and fluoroquinolones) are most effective when administered once daily achieving high serum or tissue peaks relative to the minimum inhibitory concentration (MIC) of the organism. Antimicrobials that are characterized by time-dependent killing (e.g., penicillins and cephalosporins) are most effective when the serum or tissue concentration of the drug is maintained above the MIC for an extended period of time, rather than by achieving high serum concentrations. This is achieved by either continuous infusion or prolonged infusion rates of the antimicrobial. Both types of agents are effective in the treatment of UTI, but it is important that the dose and dosing interval be determined correctly for the agent chosen to treat the infection.

The preferred route of antimicrobial administration depends on the site of infection, antimicrobial susceptibilities, the individual patient's gastrointestinal absorption and the bioavailability of the drug. Oral agents should achieve high serum and tissue concentrations for the treatment of complicated UTIs. The parenteral route should be used for empiric therapy in severely ill patients or those with poor absorption or oral bioavailability [30,31]. The selection of antimicrobial therapy should also take into account the potential toxicity and necessary dosing adjustments based on the glomerular filtration rate of the individual patient.

\subsection{Treatment Duration}

\subsubsection{Cystitis}

Recommended antimicrobial regimens and duration of therapy for acute uncomplicated cystitis are summarized in Table 2. For the treatment of uncomplicated cystitis, short-course regimens (single dose to 5 days) are recommended as first-line therapy and are as effective as longer antimicrobial regimens in achieving symptomatic cure with fewer adverse effects [2]. Recommended empirical first-line treatment regimens for uncomplicated cystitis, based on the IDSA guidelines [13], include: nitrofurantoin, trimethoprim-sulfamethoxazole (TMP-SMX), fosfomycin trometamol and pivmecillam. Nitrofurantoin monohydrate macrocrystals $100 \mathrm{mg}$ twice daily with meals for 5 days has shown good efficacy and is well tolerated with low propensity for adverse ecologic effects; it should only be used for the treatment of cystitis and avoided if pyelonephritis is suspected. TMP-SMX $160 \mathrm{mg} / 800 \mathrm{mg}$ twice daily for 3 days remains very effective with high cure 
rates [32,33] but is not recommended in areas with resistance prevalence $>20 \%[34,35]$; it is inexpensive and well tolerated with fewer ecologic adverse effects than fluoroquinolones. Fosfomycin trometamol $3 \mathrm{~g}$ sachet in a single dose or pivmecillinam $400 \mathrm{mg}$ twice daily for 3 to 7 days are also recommended as first line agents due to their low propensity for ecologic adverse effects even though in some studies they appear to be clinically inferior to TMP-SMX or fluoroquinolones [13].

Table 2. Antimicrobial management of acute uncomplicated cystitis ${ }^{\mathrm{a}}$.

\begin{tabular}{|c|c|c|}
\hline Antimicrobial & Dosing and Duration & Efficacy \\
\hline \multicolumn{3}{|c|}{ First-Line Agents } \\
\hline $\begin{array}{l}\text { Nitrofurantoin } \\
\text { monohydrate/macrocrystal }^{\text {b }}\end{array}$ & $\begin{array}{l}100 \mathrm{mg} \text { twice daily } \times 5 \text { days } \\
\text { (with meals) }\end{array}$ & $\begin{array}{l}\text { - Clinical efficacy of 5-7 day regimen: } 93 \%(84 \%-95 \%) \\
\text { - 3-day regimen appears less effective } v s . \text { longer regimens } \\
\text { - Minimal in vitro resistance }\end{array}$ \\
\hline Trimethoprim-sulfamethoxazole ${ }^{c}$ & $\begin{array}{l}160 / 800 \mathrm{mg} \text { twice-daily } \\
\times 3 \text { days }\end{array}$ & $\begin{array}{l}\text { - Clinical efficacy of 3-day TMP-SMX regimen: } \\
93 \%(90 \%-100 \%) \\
\text { Avoid if resistance }>20 \% \text { or exposure in prior } \\
\text { 3-6 months }\end{array}$ \\
\hline Fosfomycin trometamol & $3 \mathrm{~g}$ sachet in a single dose & $\begin{array}{l}\text { - Appears to be less effective vs. TMP-SMX } \\
\text { or fluoroquinolones } \\
\text { - Minimal in vitro resistance, but most labs do not test }\end{array}$ \\
\hline Pivmecillinam & $\begin{array}{l}400 \mathrm{mg} \text { twice daily } \times \\
3-7 \text { days }\end{array}$ & $\begin{array}{l}\text { - Clinical efficacy of 3-7 day regimens: } 73 \%(55 \%-82 \%) \\
\text { - Minimal in vitro resistance } \\
\text { - Unavailable in some countries }\end{array}$ \\
\hline
\end{tabular}

\begin{tabular}{|c|c|c|}
\hline \multicolumn{3}{|c|}{ Second-Line Agents } \\
\hline Fluoroquinolone: & $250 \mathrm{mg}$ twice daily $\times 3$ days & - Clinical efficacy $90 \%$ (85\%-98\%) \\
\hline Ciprofloxacin $^{\mathrm{c}}$ & $250 \mathrm{mg}$ or $500 \mathrm{mg}$ & - High prevalence of in vitro resistance in some \\
\hline Levofloxacin $^{\mathrm{c}}$ & once daily $\times 3$ days & regions of the world \\
\hline$\underline{\beta \text {-lactam }}{ }^{\text {b}}$ :(e.g., amoxicillin-clavulanate, & & - Clinical efficacy of 3-5 day regimens: $89 \%$ (79\%-98\%) \\
\hline cefdinir, cefaclor, and & $3-7$ days & - Less effective thanTMP-SMX and fluoroquinolones \\
\hline cefpodoxime-proxetil) & & - Prevalence of E. coli resistance is variable \\
\hline
\end{tabular}

Adapted from ref. [2]. ${ }^{\text {a }}$ Efficacy data and antimicrobial recommendations based on IDSA guidelines [13];

${ }^{\mathrm{b}}$ Pregnancy category B-no clear risk to fetus based on animal and/or human studies; ${ }^{\mathrm{c}}$ Pregnancy category

$\mathrm{C}$ - animal studies have shown an adverse effect on the fetus; use only if potential benefit justifies the potential risk to the fetus.

Recommended second line agents for acute uncomplicated cystitis include fluoroquinolones (levofloxacin $250 \mathrm{mg}$ or $500 \mathrm{mg}$ once daily for 3 days or ciprofloxacin $250 \mathrm{mg}$ twice daily for 3 days). Due to the rising prevalence of fluoroquinolone resistance in some regions of the world and due to their importance in treatment of a wide variety of infections, the use of fluoroquinolones should be reserved when possible for other uses than cystitis $[8,13,35]$. Beta-lactams (amoxicillin-clavulanate, cefdinir, cefaclor and cefpodoxime) for 7 days or more are also recommended as second line agents with some studies reporting lower efficacy compared to TMP-SMX and fluoroquinolones [2].

Reducing the duration of treatment and selecting recommended agents other than fluoroquinolones for the treatment of uncomplicated cystitis are important stewardship strategies. Given the ubiquity 
of cystitis, such stewardship strategies may ultimately have significant beneficial effects on antimicrobial resistance and other adverse consequences of antimicrobial therapy.

Antimicrobial-sparing strategies for the management of acute uncomplicated cystitis that warrant further study include delayed treatment [36] and the use of anti-inflammatory drugs [37].

Treatment duration for complicated cystitis has been less thoroughly studied, but in general such infections should be treated for at least 7 days, especially in men where underlying prostatic infection may exist. Although UTIs in older or pregnant women are often considered "complicated", short-course treatment has been shown to be effective in such women as mentioned earlier [20].

\subsubsection{Pyelonephritis}

The treatment of pyelonephritis following initial empiric therapy should be guided by urine culture and susceptibility results. Most episodes of acute uncomplicated pyelonephritis are treated in the outpatient setting, but patients should be hospitalized if the episode is severe, if there is hemodynamic instability, oral medications are not tolerated, poor adherence to therapy or any complicating factors such as diabetes, renal stones or pregnancy [2]. Empiric therapy for pyelonephritis should have a broad-spectrum of activity and be started without delay to avoid complications. For acute uncomplicated pyelonephritis, a fluoroquinolone is recommended as the empiric regimen of choice when feasible [13], because it is a serious infection that may be life threatening. Short-course regimens of oral levofloxacin $750 \mathrm{mg}$ once daily for 5 days appear to be effective for uncomplicated pyelonepritis and complicated UTI [13,15]. Recommended outpatient oral empiric regimens are summarized in Table 3 and include: fluoroquinolones (e.g., levofloxacin $750 \mathrm{mg}$ once daily for 5 days or ciprofloxacin $500 \mathrm{mg}$ twice daily or $1 \mathrm{~g}$ extended release daily for 7 days), TMP-SMX $160 \mathrm{mg} / 800 \mathrm{mg}$ twice daily for 7-14 days or beta-lactams for 10-14 days. A parenteral broad-spectrum agent such as ceftriaxone can be used along with these regimens if drug resistance is a concern, particularly in patients with severe infection [2,13].

Pyelonephritis in patients with "complicating" factors are at greater risk for severe complications. The optimal treatment duration is not known, and such treatment should be tailored to the severity of illness, the rapidity of response to treatment, and results of imaging studies if done. Such patients should generally be treated for 10 days or longer with antimicrobials targeted to the causative uropathogen.

In many studies the optimal duration of treatment for UTIs is defined by the absence of recurrent UTI after an arbitrary number of days (e.g., 7, 10, 14 days). Often, the minimum duration of treatment required for clinical cure is not known. To further reduce volume of consumption, selection pressure and adverse ecological effects, more studies on shorter treatments in different populations are needed [30].

\subsubsection{Catheter-Associated UTIs}

International guidelines for the management of catheter-associated UTIs (CA-UTIs) [25] recommend 7 days of antimicrobials in patients with prompt resolution of symptoms or 5 days of levofloxacin in patients who are not severely ill (assuming the organism is susceptible). Ten to 14 days of treatment are recommended for patients with a delayed response. A 3-day course of 
antimicrobial therapy could be used in women $\leq 65$ years without upper urinary tract symptoms after an indwelling catheter has been removed.

Table 3. Antimicrobial outpatient management of acute uncomplicated pyelonephritis ${ }^{\text {a }}$.

\begin{tabular}{|c|c|c|}
\hline Antimicrobial & Dosing and Duration & Efficacy \\
\hline $\begin{array}{l}\text { Fluoroquinolone: } \\
\text { Ciprofloxacin }^{\text {b }} \\
\text { Levofloxacin }^{\text {b }}\end{array}$ & $\begin{array}{l}500 \mathrm{mg} \text { orally twice-daily or } 1 \mathrm{~g} \text { extended } \\
\text { release orally once-daily } \times 7 \text { days } \\
750 \mathrm{mg} \text { orally once-daily } \times 5 \text { days }\end{array}$ & $\begin{array}{l}\text { - Clinical efficacy of ciprofloxacin } 500 \mathrm{mg} \text { orally } \\
\text { twice daily for } 7 \text { days: } 96 \% \\
\text { Clinical efficacy of levofloxacin } 750 \mathrm{mg} \text { orally or } \\
\text { intravenous once daily for } 5 \text { days: } 86 \% \text {; } v \text {. } \\
\text { ciprofloxacin } 400 \mathrm{mg} \text { intravenous or } 500 \mathrm{mg} \text { orally } \\
\text { twice daily for } \\
10 \text { days: } 81 \% \text {; most subjects in both arms received } \\
\text { oral therapy }\end{array}$ \\
\hline $\begin{array}{l}\text { Trimethoprim- } \\
\text { sulfamethoxazole }^{b}\end{array}$ & $160 / 800 \mathrm{mg}$ orally twice-daily for 14 days & $\begin{array}{l}\text { - Inferior choice for empirical therapy due to high } \\
\text { rates of resistance and corresponding failure rates } \\
\text { - Highly effective if strain susceptible } \\
\text { - E. coli resistance }>20 \% \text { in many areas of world, } \\
\text { including some areas of the US } \\
\text { - } 92 \% \text { clinical efficacy if } E \text {. coli susceptible } v s .35 \% \\
\text { if not susceptible) }\end{array}$ \\
\hline $\begin{array}{l}\text { Oral } \beta \text {-lactam } \\
\text { Specific agents are not } \\
\text { listed in IDSA guidelines }\end{array}$ & Duration $10-14$ days & $\begin{array}{l}\text { - Data limited, but inferior efficacy vs. TMP-SMX } \\
\text { and fluoroquinolones } \\
\text { - Oral } \beta \text {-lactams should be used only when other } \\
\text { recommended agents can't be used }\end{array}$ \\
\hline
\end{tabular}

Adapted from ref. [2]. ${ }^{a}$ Efficacy data and antimicrobial recommendations based on IDSA guidelines [13];

${ }^{b}$ Pregnancy category $\mathrm{C}$ - animal studies have shown an adverse effect on the fetus; use only if potential benefit justifies the potential risk to the fetus.

\subsection{Prevention Strategies}

\subsubsection{Recurrent Acute Uncomplicated Cystitis}

Several non-antimicrobial related strategies to prevent recurrent acute uncomplicated UTIs have been published [2]. Behavioral interventions include abstinence or reduction in frequency of sexual intercourse which is often not very feasible. Contraceptive methods such as spermicides and spermicide-coated condoms alter the vaginal flora and favor the colonization of uropathogens and should be avoided. Urination soon after intercourse, drinking fluids, not routinely delaying urination and wiping front to back have not been shown to be associated with a reduced risk of uncomplicated cystitis in case-control studies, but might be effective in some patients and are not unreasonable strategies to suggest for patients with recurrent cystitis. Cranberry juice, capsule or tablets are widely used by women to prevent UTI recurrences, but they have not been convincingly demonstrated to be effective in preventing such recurrences [38]. There are some small studies, however, that suggest cranberry is effective and, given that this strategy appears to be benign, it is reasonable that women continue to use cranberry if they think that it has been effective. 
Adhesion blockers such as D-mannose are increasingly being used by women to prevent cystitis, but supportive data are sparse. In a recently published randomized study [39] of 308 women with recurrent UTIs, investigators allocated patients into three groups: 2 grams of D-mannose powder in $200 \mathrm{~mL}$ of water daily, $50 \mathrm{mg}$ of daily nitrofurantoin or no treatment for 6 months. Patients in the D-mannose group and nitrofurantoin group had a significantly lower risk of recurrent UTIs during the study compared to patients receiving no prophylaxis (RR 0.239 and $0.335, p<0.0001$ ). Of concern, the authors did not present data for the D-mannose group and the nitrofurantoin group separately, although they mentioned that the difference between the two groups was not significant. Interestingly, the authors noted that the time from starting prophylaxis to onset of symptoms did not differ significantly between the groups (presumably including the no-treatment group). Patients in the D-mannose group had a significantly lower risk of side effects compared to patients in the nitrofurantoin group (RR 0.276, $p<0.0001$ ). Porru et al. [40], in a recent randomized cross-over pilot trial, evaluated the efficacy of D-mannose in the treatment and prophylaxis of recurrent UTIs in 60 patients (mean age 42 years). Patients were randomly assigned to treatment and prophylaxis with TMP-SMX or to a regimen of oral D-mannose $1 \mathrm{~g}$ every $8 \mathrm{~h}$ for 2 weeks followed by $1 \mathrm{~g}$ twice a day for 22 weeks. Patients were crossed over to the other intervention in the second phase of the study, with no further antimicrobial prophylaxis. Mean time to UTI recurrence was 52.7 days with antimicrobial treatment, and 200 days with D-mannose $(p<0.0001)$. Of note, however, the investigators used an unusual and unproven prophylactic regimen of TMP-SMX in the study (one week per month), observed a highly unusual rate of UTI recurrence in the 24-week period on TMP-SMX ( $91.7 \%$ of women had $\geq 1$ recurrence compared with $20 \%$ of the D-mannose women), and the authors do not describe how the data were analyzed for the crossover aspect of the trial. While neither of these studies provide convincing evidence that D-mannose is effective in preventing cystitis, further studies of D-mannose are clearly warranted to determine its pharmacokinetic properties and clinical efficacy.

Other non-antimicrobial strategies to reduce the risk of recurrent uncomplicated cystitis include replacement topical estrogen therapy in postmenopausal women, probiotics, oral immunostimulants and vaccination. Replacement topical estrogen normalizes the vaginal flora in postmenopausal women and has been shown to greatly reduce the risk of recurrent UTI in this population [41]. Probiotics are widely used to prevent recurrent UTI but the published data to date remain unconvincing. Probiotics are touted to protect the vagina from colonization by uropathogens by steric hindrance or blocking potential sites of attachment, production of hydrogen peroxide which is microbicidal to E. coli and other uropathogens, maintenance of a low $\mathrm{pH}$, and induction of anti-inflammatory cytokine responses in epithelial cells. However, in a review of four randomized controlled trials of lactobacillus probiotics for bacterial genitourinary infections in women, only one demonstrated a significant reduction in rates of UTI recurrence [42]. Moreover, most of these studies did not determine whether the probiotic led to vaginal colonization with the probiotic strain. While the probiotic approach has a credible scientific basis, additional adequately designed clinical trials need to be performed before its routine use can be recommended. Oral immunostimulants may have a role in UTI prevention. In a systematic review and meta-analysis of four trials that together included 891 participants, OM-89, an extract of 18 different serotypes of heat-killed uropathogenic E. coli given orally to stimulate innate immunity, decreased the rate of UTI recurrence 
(RR $0.61,95 \%$ CI $0.48-0.78$ ) [43]. The agent is commercially available in some European countries but not in the United States. Although there is great interest in developing a safe and effective UTI vaccine, there is no currently available product on the market.

Antimicrobial prevention strategies are highly effective for prevention of recurrent uncomplicated cystitis, but should be considered only as a last resort after non-antimicrobial strategies have been tried or considered and the potential risks of long term antimicrobials have been thoroughly discussed with the patient.

\subsubsection{Catheter-Associated UTIs}

Screening and treatment of patients with catheter-associated asymptomatic bacteriuria (CA-ASB) are not recommended to reduce subsequent CA-bacteriuria or CA-UTI [24]. Likewise, systemic antimicrobial treatment of ASB is not recommended to reduce the risk of symptomatic UTI in catheterized patients. The most effective way to reduce the incidence of asymptomatic or symptomatic bacteriuria is to reduce urinary catheterization by restricting its use to patients who clearly need it and by removing the catheter as soon as no longer indicated [25]. Nurse- or physician-based electronic reminders and automatic stop orders to remove unnecessary urinary catheters have been successfully implemented in clinical practice and are recommended by the IDSA guidelines. Systemic antimicrobial prophylaxis to prevent symptomatic infection should be avoided in patients with urinary catheterization in order to reduce the selection pressure for multiple-drug-resistant pathogens.

\subsection{Bacteriuria in Pregnancy}

Symptomatic and asymptomatic bacteriuria are common during pregnancy and E. coli is the most common etiologic agent. The incidence of ASB in pregnancy varies among different countries and ranges between $2 \%$ and $18 \%$ [44-46]. Studies of ASB have often been of poor quality with small sample sizes, different gestational ages, unclear definitions, differences in diagnostic techniques, timing of urine collection and different cutoff points for significant bacteriuria [47]. In both symptomatic and asymptomatic infection, quantitative culture is the gold standard for diagnosis. Current guidelines recommend screening pregnant women at least once in early pregnancy with a urine culture [22]. Treatment for ASB during pregnancy has become a standard of obstetrical care and has been shown to reduce the rate of pyelonephritis and decrease the incidence of low birth weight. However, studies of ASB in pregnancy were mostly done in the early antimicrobial era and the methodological quality of the studies limits the strength of the conclusions that can be drawn [46]. Duration of therapy for ASB should be 3-7 days [22].

There are still unknown consequences of exposing neonates to antimicrobial therapy. A long-term Danish study of 447,629 single pregnancies followed for 9.9 years found a small increased risk of epilepsy in children whose mothers received antimicrobials (mainly for UTI), including nitrofurantoin, during pregnancy [48]. Additionally, there is no clear consensus in the literature on the optimal antimicrobial choice or duration of therapy for UTI during pregnancy. In light of the possible adverse effects of antimicrobials, higher quality research is needed to better understand the direct and indirect consequences of antimicrobial exposure early in life and prudent antimicrobial use is extremely important during pregnancy and early childhood [47]. Studies exploring cost-effective 
diagnostic tools at the point of care and non-antimicrobial options to prevent or treat ASB and UTIs are needed to limit unnecessary treatment of bacteriuria in pregnancy.

\subsection{Long Term Care Facilities}

One of the most important problems in antimicrobial stewardship in LTCFs is the inappropriate use of antimicrobials to treat UTIs in asymptomatic residents [4,24]. Despite extensive research demonstrating lack of benefit and potential harm for antimicrobial use in ASB [49,50], up to $50 \%$ of asymptomatic nursing home residents are prescribed broad-spectrum antimicrobials (e.g., fluoroquinolones) for a suspected UTI [9]. In a study by Phillips et al., up to $80 \%$ of the antimicrobials prescribed to individuals with an indwelling urinary catheter were written in the absence of signs or symptoms of UTI but in the presence of urinalysis results [9]. The diagnosis of UTIs in elderly LTCF residents is challenging as there is a wide range of events that can prompt urine testing, such as changes in mental status, behaviors, color or smell of the urine with our without dysuria, or falls [51]. Increased antimicrobial stewardship efforts are indicated to reduce unnecessary urinary catheterization, unnecessary diagnostic testing and inappropriate prescribing of antimicrobials for ASB in LTCFs and other institutional settings.

Some useful strategies to improve the use of antimicrobials in LCTFs have been reported [24]. Pettersson et al. [52], described an education intervention to improve antimicrobial use in a cluster randomized trial in Swedish LTCFs including educational small group sessions with facility nurses and physicians, guidelines adapted for the local context, written materials, and feedback on prescribing. At the end of the 2 year intervention period, there was no difference between the intervention and control facilities in fluoroquinolone use for UTI. There were, however, significant differences favoring the intervention facilities in secondary outcomes, including a decrease in any antimicrobials given for all infections and an increase in a "wait and see" approach of observation with delayed empiric antimicrobials. Loeb et al. [53], in a cluster-randomized trial including 12 nursing homes evaluated the impact of implementation of consensus guidelines with treatment algorithms prior to institution of empiric antimicrobial therapy for treatment of UTIs. The intervention program included nursing education in small group interactive sessions, video tapes and written material, outreach visits and one-on-one physician detailing. Over the study period there was a significant decrease in the number of antimicrobial days given for suspected UTI in the intervention compared with control homes, but no difference between the two groups in total antimicrobial days for all indications. The difference between intervention and control groups appeared to wane over time. Zabarsky et al. [54] focused on education of healthcare providers about appropriate collection of urine specimens and not to treat ASB. Direct individual feedback regarding specific cases was given. In the six months following implementation there were significant decreases in the proportion of inappropriate urine specimens sent for culture, episodes of treatment of ASB, and total antimicrobial days. These reductions were maintained during the following 7 to 30 months. Another multicenter study in LTCFs in Finland [55] developed a program where teams comprising an infectious disease consultant, infection control nurse, and geriatrician visited 39 LTCF during 2004-2008. The site visits consisted of a structured interview concerning patients, ongoing antimicrobials, and diagnostic practices for UTI. Following the visits, regional guidelines for prudent use of antimicrobials in LTCFs were published, and the use of antimicrobials was followed up by an annual questionnaire. 
The investigators found that most of the antimicrobial were used for UTI (range by year, 66.6\%-81.1\%). At baseline, 14.5\% (177/1221) LTCF residents received antimicrobials for UTI prophylaxis and this significantly decreased to $7.8 \%(90 / 1158)(p<0.001)$ after the implementation of the multidisciplinary intervention, without an increase in the number of patients treated for acute UTI in LTCF.

\subsection{Surgical Prophylaxis}

Antimicrobials are often used to prevent specific post-operative infections. Clinical practice guidelines for antimicrobial prophylaxis in surgery [56] from the American Society of Health-System Pharmacists (ASHP), the Infectious Diseases Society of America (IDSA), the Surgical Infection Society (SIS) and the Society for Healthcare Epidemiology of America (SHEA) provide procedure-specific recommendations to avoid post-operative bacteriuria or urosepsis. The selection of prophylactic agents should be based on the individual patient's prior antimicrobial use, history of UTI and risk factors for UTI. Routine screening and treatment for ASB are discouraged in most surgical procedures as they lead to unnecessary treatment, further diagnostic testing, delays in the procedure, development of antimicrobial resistance and adverse events such as C. difficile. Drekonja et al. [57], retrospectively evaluated the use of antimicrobial treatment of ASB in 1688 patients undergoing non-urologic procedures at a single center; $25 \%$ of the patients were screened by urine culture for ASB. The authors found no difference in surgical site infection rates ( $20 \%$ vs. $16 \% ; p=0.56)$ but more frequent episodes of post-operative UTI ( $9 \%$ vs. $2 \% ; p=0.01)$ among patients treated for bacteriuria $v s$. those not treated. These findings suggest no benefit from empiric peri-operative antimicrobial therapy for ASB.

In urologic procedures such as transrectal biopsy or resection of the prostate, antimicrobial prophylaxis and treatment of bacteriuria is recommended and proven to reduce post-procedural urosepsis from $4.4 \%$ to $0.7 \%$ [58]. Herr [17] investigated 2010 consecutive patients with bladder tumors who underwent cystoscopy without antimicrobial prophylaxis at a single center by the same surgeon; $24 \%$ of the patients had documented ASB prior to the procedure. The incidence of symptomatic post-procedure UTIs within 30 days was $4.5 \%$ in colonized patients with ASB and $1.1 \%$ in uninfected patients $(p=0.02)$, all UTIs resolved within $24 \mathrm{~h}$ with oral antimicrobials. These findings suggest that ASB is common in bladder cancer patients undergoing cystoscopy, but antimicrobial prophylaxis is unnecessary because subsequent UTIs are uncommon and easily treated.

\subsection{Barriers to Guideline Implementation}

Clinicians have profound individual accountability, and yet adherence to guidelines at the bedside often remains low causing omission of therapies contributing to preventable harm, suboptimal outcomes and waste of resources [59]. The reasons for poor compliance with guidelines are multifactorial. Several authors have proposed steps to overcome the barriers in guideline implementation, including more transparency in the level of recommendations, prioritizing which therapies have the greatest benefit to the patients at the lowest risks and costs, and implementation of order sets at the point of care incorporating the recommendations from national guidelines [60,61]. Henig et al. [62], systematically evaluated the methodological quality of eight national and international guidelines 
for the treatment of UTIs in adults published in the last 10 years (2004-2013); the authors identified variable recommendations depending on local epidemiology and different methodological rigor in guideline development. Some limitations to the UTI guidelines include poor descriptions of applicability such as likely barriers and facilitators to implementation, strategies to improve update and resource implications, lack of patient involvement in the development of recommendations and none of the published guidelines used the GRADE methodology to interpret the evidence and grade the recommendations [62]. Existing guidelines for the treatment of UTIs rarely address the implementation of recommendations within antimicrobial stewardship programs.

\subsection{Areas of Uncertainty}

More research is needed to optimize the diagnosis, treatment and prevention strategies of UTIs. Targeted rapid diagnostic tests that could distinguish between inflammation and infection, identify the pathogen and its mechanisms of antimicrobial resistance are very much needed. The development of newer antimicrobial oral agents with novel mechanisms of action against Gram-negative organisms to treat uropathogens is also awaited. Faster diagnostics and better antimicrobials will not improve antimicrobial prescribing practices unless global efforts continue to reinforce the importance of prudent, thoughtful and rational use of antimicrobials.

\section{Conclusions and Recommendations}

The diagnosis of UTI is primarily based on signs and symptoms rather than isolated laboratory findings. Urine cultures are often not useful for acute uncomplicated cystitis, are recommended for patients with uncomplicated pyelonephritis and complicated UTI, and with few exceptions, should not be collected in asymptomatic patients. Antimicrobial therapy should be tailored to each patient taking into consideration the severity of disease, individual and local patterns of antimicrobial resistance and the potential for collateral damage associated with antimicrobial use. Selecting the correct drug, dose, and shortest clinically effective duration of therapy when possible, is key to optimal antimicrobial stewardship. Strategies to prevent recurrent UTIs and catheter-associated bacteriuria could greatly reduce the use of antimicrobials and are therefore key stewardship modalities. It is the responsibility of all healthcare providers to practice antimicrobial stewardship and prescribe antimicrobials prudently, thoughtfully and rationally.

\section{Author Contributions}

Each author has contributed to the literature search, drafting and review of the manuscript.

\section{Conflicts of Interest}

The authors declare no conflict of interest.

\section{References}

1. Schappert, S.M.; Rechtsteiner, E.A. Ambulatory medical care utilization estimates for 2007. Vital Health Stat. 2011, 13, 1-38. 
2. Hooton, T.M. Clinical practice. Uncomplicated urinary tract infection. N. Engl. J. Med. 2012, $366,1028-1037$.

3. Centers for Disease Control and Prevention, Healthcare-Associated Infections. Catheter Associated Urinary Tract Infections. Available online: http://www.cdc.gov/HAI/ca_uti/ uti.html/ (accessed on 7 March 2014).

4. Nicolle, L.E. Urinary tract infections in long-term-care facilities. Infect. Control Hosp. Epidemiol. 2001, 22, 167-175.

5. Sammon, J.D.; Sharma, P.; Rahbar, H.; Roghmann, F.; Ghani, K.R.; Sukumar, S.; Karakiewicz, P.I.; Peabody, J.O.; Elder, J.S.; Menon, M.; et al. Predictors of admission in patients presenting to the emergency department with urinary tract infection. World J. Urol. 2013, doi:10.1007/s00345-013-1167-3.

6. Coupat, C.; Pradier, C.; Degand, N.; Hofliger, P.; Pulcini, C. Selective reporting of antibiotic susceptibility data improves the appropriateness of intended antibiotic prescriptions in urinary tract infections: A case-vignette randomised study. Eur. J. Clin. Microbiol. Infect. Dis. 2013, 32, 627-636.

7. Baron, E.J. The role of the clinical microbiology laboratory in the diagnosis of selected infectious processes. J. Clin. Microbiol. 2011, 49, S25.

8. Scottish Intercollegiate Guidelines Network (SIGN). Management of Suspected Bacterial Urinary Tract Infections in Adults. Available online: http://www.sign.ac.uk/pdf/sign88.pdf (accessed on 24 February 2014).

9. Phillips, C.D.; Adepoju, O.; Stone, N.; Moudouni, D.K.; Nwaiwu, O.; Zhao, H.; Frentzel, E.; Mehr, D.; Garfinkel, S. Asymptomatic bacteriuria, antibiotic use, and suspected urinary tract infections in four nursing homes. BMC Geriatr. 2012, 12, e73.

10. Tremolieres, F. Short or long course antibiotics. Is there a debate on the duration of treatment? Presse Med. 2002, 31, 1495-1501.

11. Duc Volluz, S.; Abbet, P.; Troillet, N. What is the optimal duration of antibiotics in common infections? Rev. Med. Suisse 2010, 6, 1901-1905.

12. Dryden, M.; Johnson, A.P.; Ashiru-Oredope, D.; Sharland, M. Using antibiotics responsibly: Right drug, right time, right dose, right duration. J. Antimicrob. Chemother. 2011, 66, 2441-2443.

13. Gupta, K.; Hooton, T.M.; Naber, K.G.; Wullt, B.; Colgan, R.; Miller, L.G.; Moran, G.J.; Nicolle, L.E.; Raz, R.; Schaeffer, A.J.; et al. International clinical practice guidelines for the treatment of acute uncomplicated cystitis and pyelonephritis in women: A 2010 update by the infectious diseases society of america and the european society for microbiology and infectious diseases. Clin. Infect. Dis. 2011, 52, e103-e120.

14. Fitzgerald, A.; Mori, R.; Lakhanpaul, M.; Tullus, K. Antibiotics for treating lower urinary tract infection in children. Cochrane Database Syst. Rev. 2012, 8, CD006857.

15. Klausner, H.A.; Brown, P.; Peterson, J.; Kaul, S.; Khashab, M.; Fisher, A.C.; Kahn, J.B. A trial of levofloxacin $750 \mathrm{mg}$ once daily for 5 days versus ciprofloxacin $400 \mathrm{mg}$ and/or $500 \mathrm{mg}$ twice daily for 10 days in the treatment of acute pyelonephritis. Curr. Med. Res. Opin. 2007, 23, 2637-2645. 
16. Talan, D.A.; Stamm, W.E.; Hooton, T.M.; Moran, G.J.; Burke, T.; Iravani, A.; Reuning-Scherer, J.; Church, D.A. Comparison of ciprofloxacin (7 days) and trimethoprim-sulfamethoxazole (14 days) for acute uncomplicated pyelonephritis pyelonephritis in women: A randomized trial. JAMA 2000, 283, 1583-1590.

17. Herr, H.W. Should antibiotics be given prior to outpatient cystoscopy? A plea to urologists to practice antibiotic stewardship. Eur. Urol. 2013, doi:10.1016/j.eururo.2013.08.054.

18. Hyun, D.Y.; Hersh, A.L.; Namtu, K.; Palazzi, D.L.; Maples, H.D.; Newland, J.G.; Saiman, L. Antimicrobial stewardship in pediatrics: How every pediatrician can be a steward. JAMA Pediatr. 2013, 167, 859-866.

19. Lutters, M.; Vogt-Ferrier, N.B. Antibiotic duration for treating uncomplicated, symptomatic lower urinary tract infections in elderly women. Cochrane Database Syst. Rev. 2008, doi:10.1002/14651858.CD001535.

20. Vazquez, J.C.; Abalos, E. Treatments for symptomatic urinary tract infections during pregnancy. Cochrane Database Syst. Rev. 2011, doi:10.1002/14651858.CD002256.

21. Johansen, T.E.; Botto, H.; Cek, M.; Grabe, M.; Tenke, P.; Wagenlehner, F.M.; Naber, K.G. Critical review of current definitions of urinary tract infections and proposal of an EAU/ESIU classification system. Int. J. Antimicrob. Agents 2011, 38, 64-70.

22. Nicolle, L.E.; Bradley, S.; Colgan, R.; Rice, J.C.; Schaeffer, A.; Hooton, T.M. Infectious diseases society of america guidelines for the diagnosis and treatment of asymptomatic bacteriuria in adults. Clin. Infect. Dis. 2005, 40, 643-654.

23. Bent, S.; Nallamothu, B.K.; Simel, D.L.; Fihn, S.D.; Saint, S. Does this woman have an acute uncomplicated urinary tract infection? JAMA 2002, 287, 2701-2710.

24. Nicolle, L.E. Antimicrobial stewardship in long term care facilities: What is effective? Antimicrob. Resist Infect. Control 2014, doi:10.1186/2047-2994-3-6.

25. Hooton, T.M.; Bradley, S.F.; Cardenas, D.D.; Colgan, R.; Geerlings, S.E.; Rice, J.C.; Saint, S.; Schaeffer, A.J.; Tambayh, P.A.; Tenke, P.; et al. Diagnosis, prevention, and treatment of catheter-associated urinary tract infection in adults: 2009 international clinical practice guidelines from the infectious diseases society of america. Clin. Infect. Dis. 2010, 50, 625-663.

26. Gupta, K.; Hooton, T.M.; Miller, L. Managing uncomplicated urinary tract infection-Making sense out of resistance data. Clin. Infect. Dis. 2011, 53, 1041-1042.

27. Hooton, T.M.; Roberts, P.L.; Cox, M.E.; Stapleton, A.E. Voided midstream urine culture and acute cystitis in premenopausal women. N. Engl. J. Med. 2013, 369, 1883-1891.

28. Tan, T.Y.; McNulty, C.; Charlett, A.; Nessa, N.; Kelly, C.; Beswick, T. Laboratory antibiotic susceptibility reporting and antibiotic prescribing in general practice. J. Antimicrob. Chemother. 2003, 51, 379-384.

29. McNulty, C.A.; Lasseter, G.M.; Charlett, A.; Lovering, A.; Howell-Jones, R.; Macgowan, A.; Thomas, M. Does laboratory antibiotic susceptibility reporting influence primary care prescribing in urinary tract infection and other infections? J. Antimicrob. Chemother. 2011, 66, 1396-1404.

30. Gyssens, I.C. Antibiotic policy. Int. J. Antimicrob. Agents 2011, 38, 11-20. 
31. Dellit, T.H.; Owens, R.C.; McGowan, J.E., Jr.; Gerding, D.N.; Weinstein, R.A.; Burke, J.P.; Huskins, W.C.; Paterson, D.L.; Fishman, N.O.; Carpenter, C.F.; et al. Infectious diseases society of america and the society for healthcare epidemiology of america guidelines for developing an institutional program to enhance antimicrobial stewardship. Clin. Infect. Dis. 2007, 44, 159-177.

32. Fair, W.R.; Crane, D.B.; Peterson, L.J.; Dahmer, C.; Tague, B.; Amos, W. Three-day treatment of urinary tract infections. J. Urol. 1980, 123, 717-721.

33. Kavatha, D.; Giamarellou, H.; Alexiou, Z.; Vlachogiannis, N.; Pentea, S.; Gozadinos, T.; Poulakou, G.; Hatzipapas, A.; Koratzanis, G. Cefpodoxime-proxetil versus trimethoprim-sulfamethoxazole for short-term therapy of uncomplicated acute cystitis in women. Antimicrob. Agents Chemother. 2003, 47, 897-900.

34. Raz, R.; Chazan, B.; Kennes, Y.; Colodner, R.; Rottensterich, E.; Dan, M.; Lavi, I.; Stamm, W. Empiric use of trimethoprim-sulfamethoxazole (tmp-smx) in the treatment of women with uncomplicated urinary tract infections, in a geographical area with a high prevalence of tmp-smx-resistant uropathogens. Clin. Infect. Dis. 2002, 34, 1165-1169.

35. Gupta, K.; Hooton, T.M.; Stamm, W.E. Increasing antimicrobial resistance and the management of uncomplicated community-acquired urinary tract infections. Ann. Int. Med. 2001, 135, 41-50.

36. Little, P.; Moore, M.V.; Turner, S.; Rumsby, K.; Warner, G.; Lowes, J.A.; Smith, H.; Hawke, C.; Leydon, G.; Arscott, A.; et al. Effectiveness of five different approaches in management of urinary tract infection: Randomised controlled trial. Br. Med. J. 2010, 340, c199.

37. Bleidorn, J.; Gagyor, I.; Kochen, M.M.; Wegscheider, K.; Hummers-Pradier, E. Symptomatic treatment (ibuprofen) or antibiotics (ciprofloxacin) for uncomplicated urinary tract infection?-Results of a randomized controlled pilot trial. BMC Med. 2010, doi:10.1186/1741-7015-8-30.

38. Barbosa-Cesnik, C.; Brown, M.B.; Buxton, M.; Zhang, L.; DeBusscher, J.; Foxman, B. Cranberry juice fails to prevent recurrent urinary tract infection: Results from a randomized placebo-controlled trial. Clin. Infect. Dis. 2011, 52, 23-30.

39. Kranjeec, B.; Papes, D.; Altarac, S. D-Mannose powder for prophylaxis of recurrent urinary tract infections in women: A randomized clinical trial. World J. Urol. 2014, 32, 79-84.

40. Porru, D.; Parmigiani, A.; Tinelli, C.; Barletta, D.; Choussos, D.; di Franco, C.; Bobbi, V.; Bassi, S.; Miller, O.; Gardella, B.; et al. Oral D-mannose in recurrent urinary tract infections in women: A pilot study. J. Clin. Urol. 2014, doi:10.1177/2051415813518332.

41. Raz, R.; Stamm, W.E. A controlled trial of intravaginal estriol in postmenopausal women with recurrent urinary tract infections. N. Engl. J. Med. 1993, 329, 753-756.

42. Barrons, R.; Tassone, D. Use of lactobacillus probiotics for bacterial genitourinary infections in women: A review. Clin. Ther. 2008, 30, 453-468.

43. Beerepoot, M.A.; Geerlings, S.E.; van Haarst, E.P.; van Charante, N.M.; ter Riet, G. Nonantibiotic prophylaxis for recurrent urinary tract infections: A systematic review and meta-analysis of randomized controlled trials. J. Urol. 2013, 190, 1981-1989. 
44. Jain, V.; Das, V.; Agarwal, A.; Pandey, A. Asymptomatic bacteriuria \& obstetric outcome following treatment in early versus late pregnancy in north indian women. Indian J. Med. Res. 2013, 137, 753-758.

45. Bruce, F.C.; Berg, C.J.; Joski, P.J.; Roblin, D.W.; Callaghan, W.M.; Bulkley, J.E.; Bachman, D.J.; Hornbrook, M.C. Extent of maternal morbidity in a managed care population in Georgia. Paediatr. Perinat. Epidemiol. 2012, 26, 497-505.

46. Schnarr, J.; Smaill, F. Asymptomatic bacteriuria and symptomatic urinary tract infections in pregnancy. Eur. J. Clin. Invest. 2008, 38, 50-57.

47. Schneeberger, C.; Kazemier, B.M.; Geerlings, S.E. Asymptomatic bacteriuria and urinary tract infections in special patient groups: Women with diabetes mellitus and pregnant women. Curr. Opin. Infect. Dis. 2014, 27, 108-114.

48. Miller, J.E.; Pedersen, L.H.; Sun, Y.; Olsen, J. Maternal use of cystitis medication and childhood epilepsy in a danish population-based cohort. Paediatr. Perinat. Epidemiol. 2012, 26, 589-595.

49. Yoshikawa, T.T. Antimicrobial resistance and aging: Beginning of the end of the antibiotic era? J. Am. Geriatr. Soc. 2002, 50, S226-S229.

50. Wiener, J.; Quinn, J.P.; Bradford, P.A.; Goering, R.V.; Nathan, C.; Bush, K.; Weinstein, R.A. Multiple antibiotic-resistant Klebsiella and Escherichia coli in nursing homes. JAMA 1999, 281, 517-523.

51. Juthani-Mehta, M.; Quagliarello, V.; Perrelli, E.; Towle, V.; van Ness, P.H.; Tinetti, M. Clinical features to identify urinary tract infection in nursing home residents: A cohort study. J. Am. Geriatr. Soc. 2009, 57, 963-970.

52. Pettersson, E.; Vernby, A.; Molstad, S.; Lundborg, C.S. Can a multifaceted educational intervention targeting both nurses and physicians change the prescribing of antibiotics to nursing home residents? A cluster randomized controlled trial. J. Antimicrob. Chemother. 2011, 66, 2659-2666.

53. Loeb, M.; Brazil, K.; Lohfeld, L.; McGeer, A.; Simor, A.; Stevenson, K.; Zoutman, D.; Smith, S.; Liu, X.; Walter, S.D. Effect of a multifaceted intervention on number of antimicrobial prescriptions for suspected urinary tract infections in residents of nursing homes: Cluster randomised controlled trial. Br. Med. J. 2005, 331, e669.

54. Zabarsky, T.F.; Sethi, A.K.; Donskey, C.J. Sustained reduction in inappropriate treatment of asymptomatic bacteriuria in a long-term care facility through an educational intervention. Am. J. Infect. Control 2008, 36, 476-480.

55. Rummukainen, M.L.; Jakobsson, A.; Matsinen, M.; Jarvenpaa, S.; Nissinen, A.; Karppi, P.; Lyytikainen, $\mathrm{O}$. Reduction in inappropriate prevention of urinary tract infections in long-term care facilities. Am. J. Infect. Control 2012, 40, 711-714.

56. Bratzler, D.W.; Dellinger, E.P.; Olsen, K.M.; Perl, T.M.; Auwaerter, P.G.; Bolon, M.K.; Fish, D.N.; Napolitano, L.M.; Sawyer, R.G.; Slain, D.; et al. Clinical practice guidelines for antimicrobial prophylaxis in surgery. Am. J. Health Syst. Pharm. 2013, 70, 195-283.

57. Drekonja, D.M.; Zarmbinski, B.; Johnson, J.R. Preoperative urine cultures at a veterans affairs medical center. JAMA Int. Med. 2013, 173, 71-72. 
58. Berry, A.; Barratt, A. Prophylactic antibiotic use in transurethral prostatic resection: A meta-analysis. J. Urol. 2002, 167, 571-577.

59. Pronovost, P.J. Enhancing physicians' use of clinical guidelines. JAMA 2013, 310, 2501-2502.

60. Cabana, M.D.; Rand, C.S.; Powe, N.R.; Wu, A.W.; Wilson, M.H.; Abboud, P.A.; Rubin, H.R. Why don't physicians follow clinical practice guidelines? A framework for improvement. JAMA 1999, 282, 1458-1465.

61. Hecker, M.T.; Fox, C.J.; Son, A.H.; Cydulka, R.K.; Siff, J.E.; Emerman, C.L.; Sethi, A.K.; Muganda, C.P.; Donskey, C.J. Effect of a stewardship intervention on adherence to uncomplicated cystitis and pyelonephritis guidelines in an emergency department setting. PLoS One 2014, 9, e87899.

62. Henig, O.; Yahav, D.; Leibovici, L.; Paul, M. Guidelines for the treatment of pneumonia and urinary tract infections: Evaluation of methodological quality using the appraisal of guidelines, research and evaluation II instrument. Clin. Microbiol. Infect. 2013, 19, 1106-1114. 


\title{
Chapter 2
}

\section{Anti-Adhesion Activity of A2-type Proanthocyanidins (a Cranberry Major Component) on Uropathogenic E. coli and $P$. mirabilis Strains}

\section{Daria Nicolosi, Gianna Tempera, Carlo Genovese and Pio M. Furneri}

\begin{abstract}
Urinary tract infections (UTIs) are relatively common in women and may be classified as uncomplicated or complicated, depending upon the urinary tract anatomy and physiology. Acute uncomplicated cystitis (AUC) occurs when urinary pathogens from the bowel or vagina colonize the periurethral mucosa and reach the bladder. The vast majority of episodes in healthy women involving the same bacterial strain that caused the initial infection are thought to be reinfections. About $90 \%$ of AUC are caused by uropathogenic Escherichia coli (UPEC), but Proteus mirabilis also plays an important role. Several studies support the importance of cranberry (Vaccinium macrocarpon) proanthocyanidins in preventing adhesion of P-fimbriated UPEC to uroepithelial cells. In this study, we evaluated the in vitro anti-adhesion activity of A2-linked proanthocyanidins from cranberry on a UPEC and Proteus mirabilis strains and their possible influence on urease activity of the latter. A significant reduction of UPEC adhesion (up to 75\%) on the HT1376 cell line was observed vs. control. For the strains of $P$. mirabilis there was also a reduction of adhesion (up to $75 \%$ ) compared to controls, as well as a reduction in motility and urease activity. These results suggest that A2-type cranberry proanthocyanidins could aid in maintaining urinary tract health.
\end{abstract}

Reprinted from Antibiotics. Cite as: Nicolosi, D.; Tempera, G.; Genovese, C.; Furneri, P.M. Anti-Adhesion Activity of A2-type Proanthocyanidins (a Cranberry Major Component) on Uropathogenic E. coli and P. mirabilis Strains. Antibiotics 2014, 3, 143-154.

\section{Introduction}

The infections of the urinary tract (UTI) are very common pathologies, both in hospitals and in the community [1]. Community infections are defined as uncomplicated when they are limited to only the lower urinary tract without anomalies; these mainly affect young women due to their anatomy (short urethra with respect to men and easy colonization of the periurethral zone by intestinal bacteria). From $25 \%$ to $50 \%$ of young women have at least one episode of UTIs in their lives. Of these, about $27 \%$ have a relapse during the six months following the first infection, while about 3\% have two relapses during the same period [2].

The symptomatology is the same as the first infection: frequent and intense need to urinate, burning and/or pain on urination. The urine is often cloudy, sometimes even pink due to the presence of blood. 
Among the risk factors that predispose women to contract recurrent cystitis are frequent sexual relations, having contracted their first UTI at less than 15 years of age and a family history of UTIs. Some studies have also shown that when the first infection is due to E. coli, there is a greater probability of having a relapse in the following six months than when the infection is due to other microorganisms [3]. Numerous data suggest that an alteration of the normal vaginal microbiota, in particular when there is a reduction of the lactobacillus population with consequent lowering of the quantity of hydrogen peroxide produced, can be considered a predisposing factor for $E$. coli colonization. Also, for this reason, post-menopausal women can develop recurrent cystitis. The risk factors are the same as those for younger women, with the addition that the lowering of estrogens following menopause causes notable modifications of the vaginal microbiota, in particular a loss of lactobacilli [4]. Recurrent urinary infections have a negative impact on the quality of life of "predisposed" women, both in psychological and economical terms. A woman with recurrent cystitis has to have frequent urine tests, antibiotic treatment and, when prescribed, also preventive therapy.

Preventive antibiotic treatment, which should be started only after having eradicated the pre-existing infection, is carried out, following guidelines, with trimethoprim-sulfamethoxazole, nitrofurantoin, cefaclor or cefalexin, ciprofloxacin or norfloxacin, and fosfomycin. These antibiotics are used at lower doses with respect to those recommended for the treatment of the full-blown infection [5]. Most clinicians recommend continuing therapy for at least six months. Even if these antibiotics are well tolerated over the long-term, the emergence of resistant strains is always a big problem, especially as regards the widely-used broad spectrum antibiotics such as fluoroquinolones [6]. There are, in fact, many on-going studies trying to establish the real contribution of long-term antibiotic therapy to the emergence of multi-resistant strains.

Uropathogenic Escherichia coli (UPEC) is the etiological agent in about $90 \%$ of community acquired infections and more than $50 \%$ of those acquired in hospitals, including those associated with the presence of a catheter. The uropathogenic strains of E. coli can be classified in four phylogentic groups called A, B1, B2 and D. Principally, B2 and D cause most extra-intestinal infections, including UTIs. These strains differ from those believed to be harmless commensals of the intestine due to the expression of particular virulence factors that specifically enhance their ability to cause infections of the human urinary tract. Most strains express numerous fimbriae (also called adhesins) diffused on the cell surface, belonging principally to two groups: type 1 fimbriae, mannose-sensitive (MS), which bind to the glycoproteins in the mannose (the virulence factors most frequently expressed by $80 \%$ to $100 \%$ of the UPEC strains), and P fimbriae, mannose-resistant (MR), which, instead, bind to the $\alpha-\mathrm{D}-\mathrm{Gal}(1,4)-\beta-\mathrm{D}-\mathrm{Gal}$, a disaccharide of galactose. Adhesins are necessary for the UPEC strains to bind to uroepithelial cells or, eventually, to catheter surfaces. Type 1 fimbriae are those that mediate the initial phases of urinary infection in as much as they use the property of the vesicle mucous, rich in mannose receptors. It is believed that these adhesins are also able to recognize the extra-cellular matrix proteins (collagen, fibronectin, laminin and Tamm-Horsifall proteins) thus distinguishing epithelia from other structures. It also appears that these adhesins can mediate bacterial auto-aggregation and the formation of biofilm that are inducers of the inflammatory response associated with adhesion and to the colonization of UPEC strains.

The P fimbriae, the second most common virulence factor associated with UPEC strains, are associated with the process of invasivity, in as much as they would come into play later to guarantee 
mannose-independent adhesion, necessary to stop bacteria being eliminated together with the mucous in the urine.

Among the other Gram-negatives there is the species Proteus, which are not included among the most common causes of UTIs but play an important role in catheterized patients or those having structural anomalies of the urinary tract. Proteus has many virulence factors that include, among others, the notable motility provided by flagella, the production of urease and adhesion to uroepithelial cells mediated by fimbriae [7]. P. mirabilis expresses different types of fimbriae, among which are those that are mannose-resistant [8,9]. Proteus, instead, is particularly important for its ability to form calcium and magnesium (struvite) stones in the bladder and kidneys following hydrolysis of urea catalyzed by urease [10], and biofilm that is particularly stabile thanks to the action of various fimbriae $[11,12]$.

Numerous studies have recently shown a correlation between the consumption of cranberries (Vaccinum macrocarpon) and the possibility of preventing UTIs [13-17]. The mechanism by which cranberries are effective in the prevention and treatment of UTIs has not yet been definitively established, but it seems very probable that it interferes with the mechanisms of bacterial adhesion to uroepithelial cells. If the bacteria are not able to adhere they are not able to colonize the urinary tract. This effect is to be attributed to two components of cranberries: fructose, that blocks Type 1 fimbriae (sensitive to mannose), and the proanthocyanidins (PACs) that, instead, inhibit the P fimbriae (mannose resistant). PACs can be of type A or B, but only those of type A (contained in cranberries) have been associated with anti-adhesive activity against uropathogens [18].

Cranberries are commercially available as: juice, syrup, capsules and lozenges. Their preparation can modify the composition and the contents of PACs [12]. For this reason, in this study we decided to use pure type A2 PACs, in the quantities indicated as effective: $50 \mu \mathrm{g} / \mathrm{mL}$ [19] and at higher and lower dosages ranging from $15 \mu \mathrm{g} / \mathrm{mL}$ to $100 \mu \mathrm{g} / \mathrm{mL}$.

The aim of our study was to evaluate the inhibiting activity of type A2 proanthocyanidins on strains of Proteus, isolated from urine, compared to the already well-known activity on uropathogenic strains of E. coli. We also evaluated the effect of type A PACs on the motility of Proteus and on the production of urease.

\section{Results and Discussion}

\subsection{Adhesion Assay}

The assay of adhesion carried out on the strains treated with PACs showed a reduction of up to $75 \%$ of the adhesion index both for the UPEC strains and for the strains of P. mirabilis, with respect to the same strains cultured without PACs. Such a large reduction was obtained only at the concentration of $50 \mu \mathrm{g} / \mathrm{mL}$. The lower concentrations had a variable efficacy in reducing adhesion of the bacteria in the study, as well as providing discrepant results. At the concentration of $15 \mu \mathrm{g} / \mathrm{mL}$, E. coli shows a decrease between $13.8 \%$ and $24.1 \%$, while P. mirabilis presents a high variability in its values, with percentages ranging from $3.3 \%$ up to $49.6 \%$. With $25 \mu \mathrm{g} / \mathrm{mL}$, a decrease between $36.9 \%$ and $53.6 \%$ for $E$. coli and between $44.9 \%$ and $68.4 \%$ for $P$. mirabilis occurs. With $50 \mu \mathrm{g} / \mathrm{mL}$, values appear more consistent, with reduction percentages varying from $67.1 \%$ to $75.4 \%$ for E. coli and from $61.4 \%$ to $75.4 \%$ for P. mirabilis. With $75 \mu \mathrm{g} / \mathrm{mL}$, the observed 
decrease is between $74.3 \%$ to $79.9 \%$ for $E$. coli and from $66.7 \%$ to $77.4 \%$ for per P. mirabilis. At the concentration of $100 \mu \mathrm{g} / \mathrm{mL}$, the reduction goes from $78 \%$ up to $84.8 \%$ for E. coli and from $77.6 \%$ up to $81.7 \%$ for $P$. mirabilis. These results are shown in Figures 1 and 2 demonstrating the constant decrease of adhesion by all the strains examined. The count of adhering bacteria was carried out manually, both for controls (Figure 3) and treated strains (Figure 4).

Figure 1. Adhesion indexes for the strains of E. coli.

\section{E. Coli}

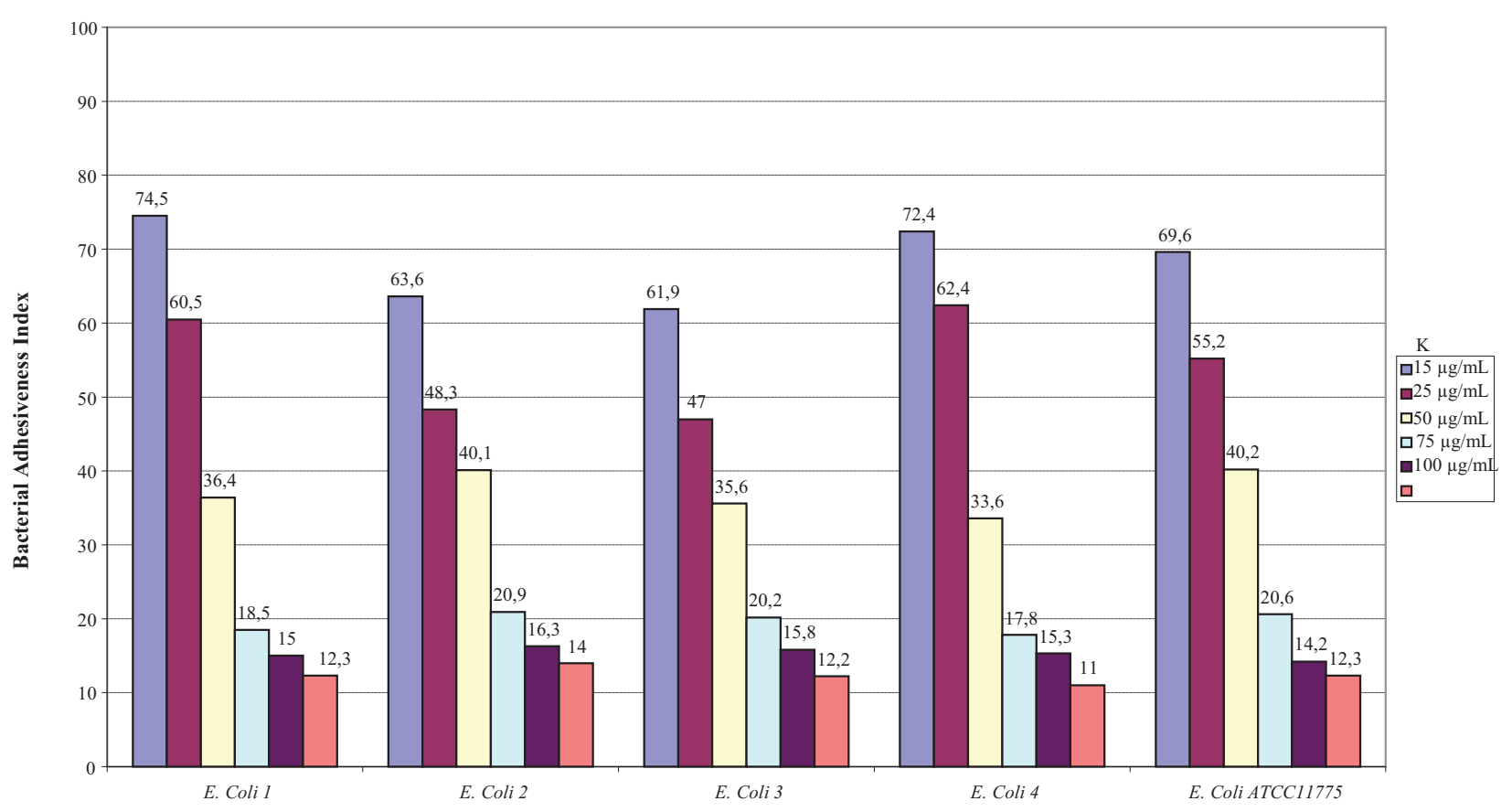

Figure 2. Adhesion indexes for the strains of P.mirabilis. In both figures the reduction of the adhesion indexes is clear.

\section{P. Mirabilis}

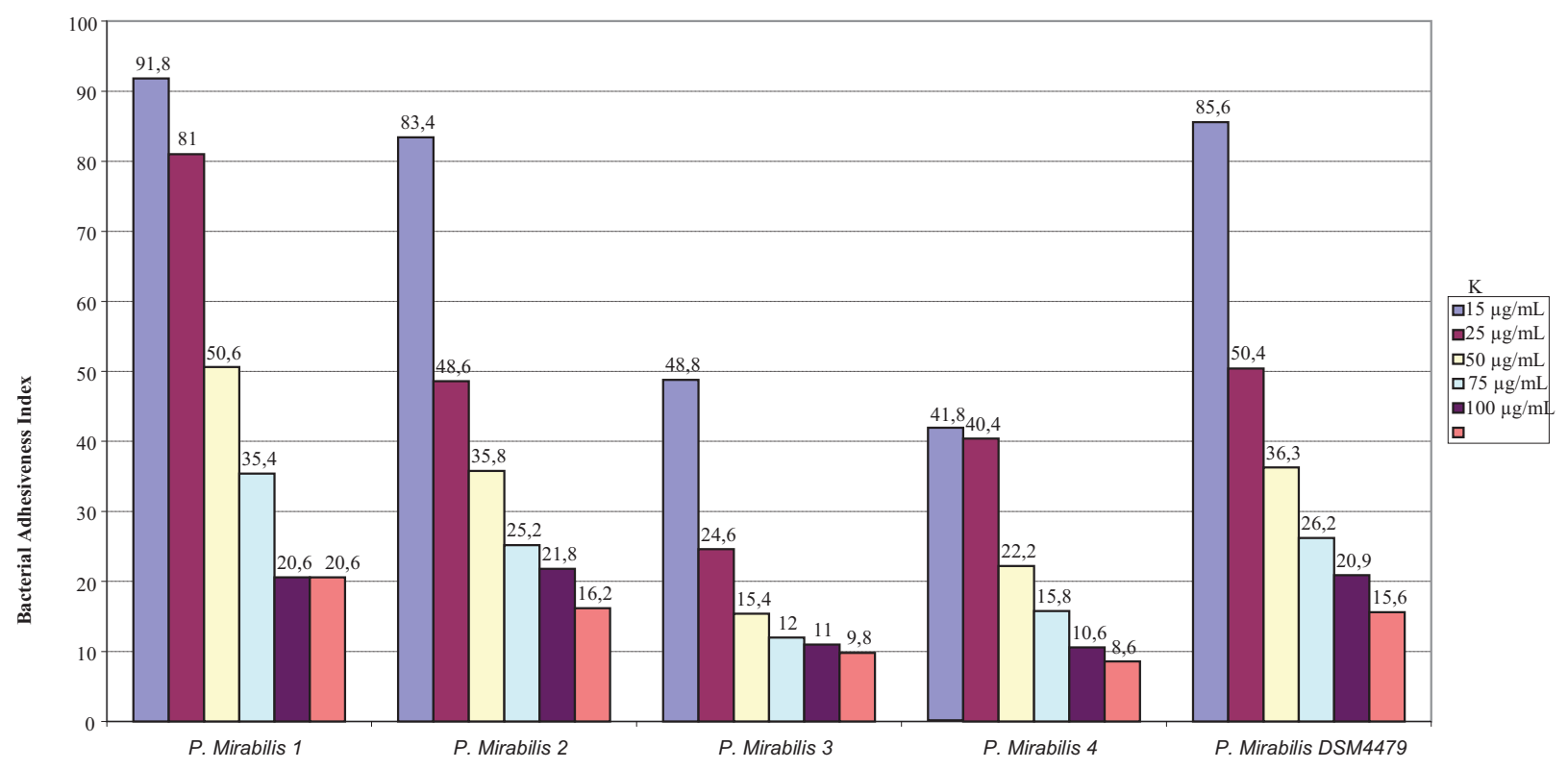


Figure 3. (a)(b) P. mirabilis adherent to a cell on a control slide.

(a)

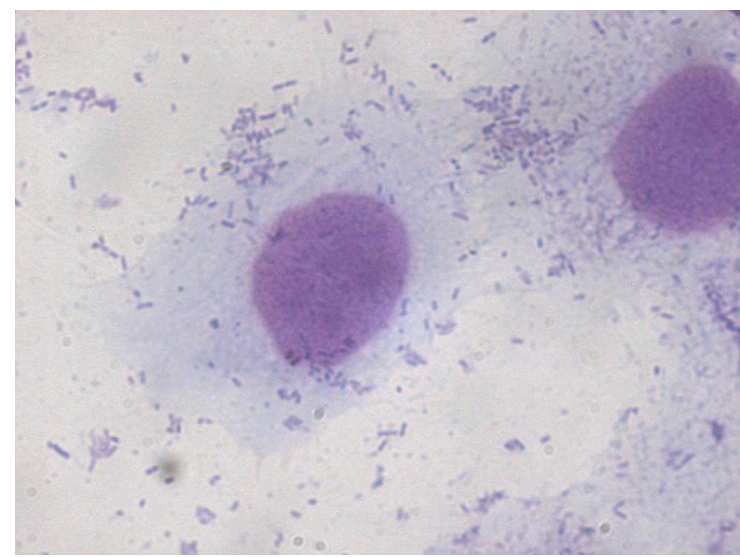

(b)

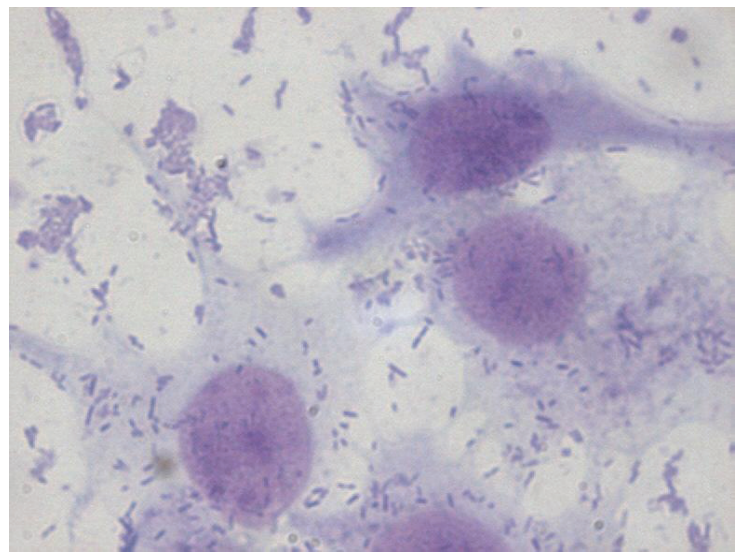

Figure 4. (a-c) Strains of $P$. mirabilis adherent to cells after treatment with PACs at the concentration of $50 \mu \mathrm{g} / \mathrm{mL}$. The lower number of adherent bacteria can be clearly seen.

(a)

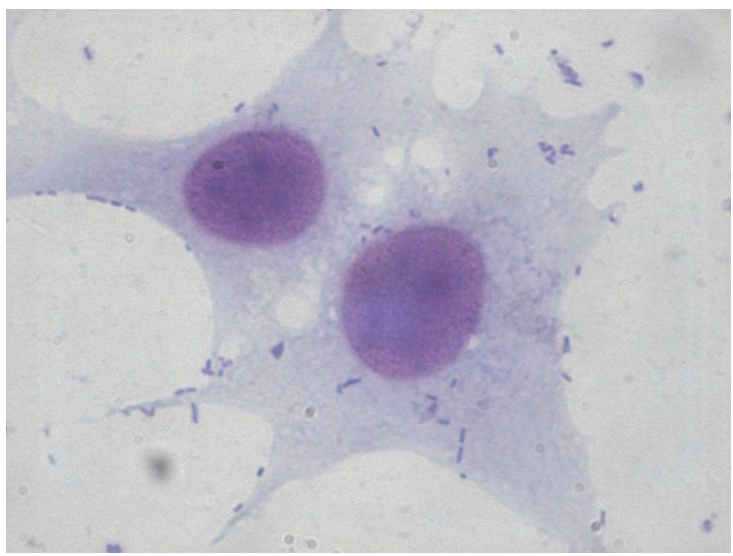

(b)

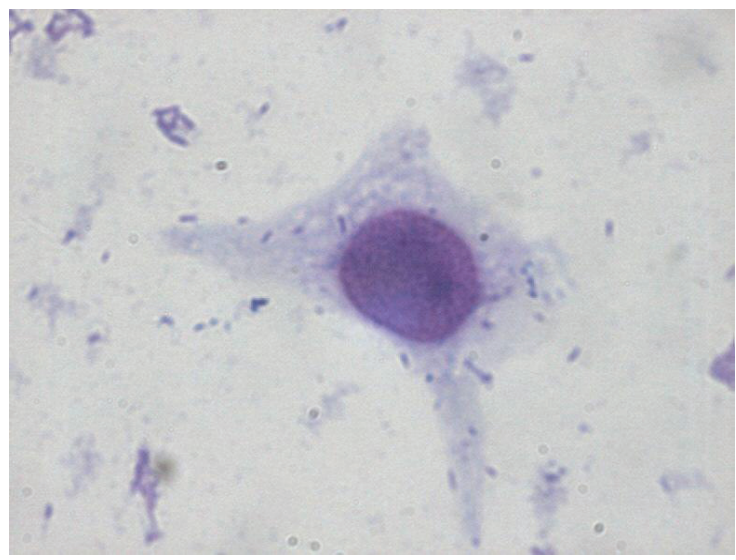

(c)

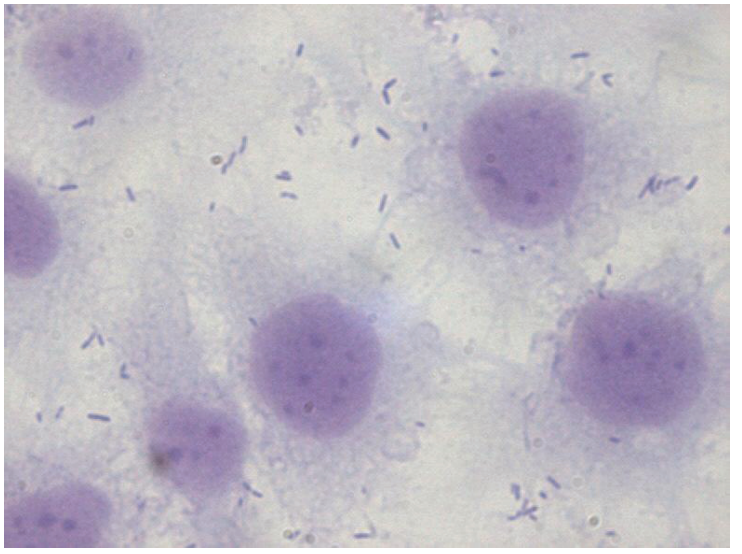

\subsection{Scanning Electron Microscopy}

From the images obtained from scanning electron microscopy, the cells are integral and the adherent bacteria are fewer than those in the controls (Figure 5). 
Figure 5. (a,b) A cell with few adhering bacteria at the concentration of $50 \mu \mathrm{g} / \mathrm{mL}$. In the outer part, which is in contact with the environment, the cells have cilia, flagella and microvilli; (c) In the controls the cells are also integral but with a higher number of adherent bacteria.

(a)

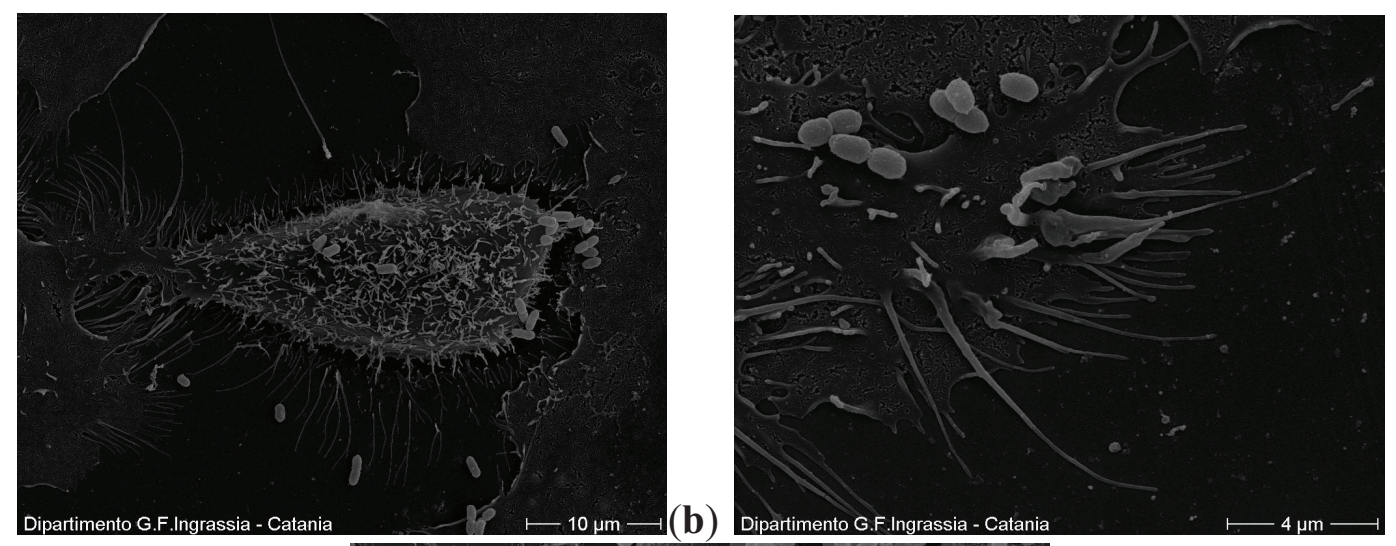

(c)

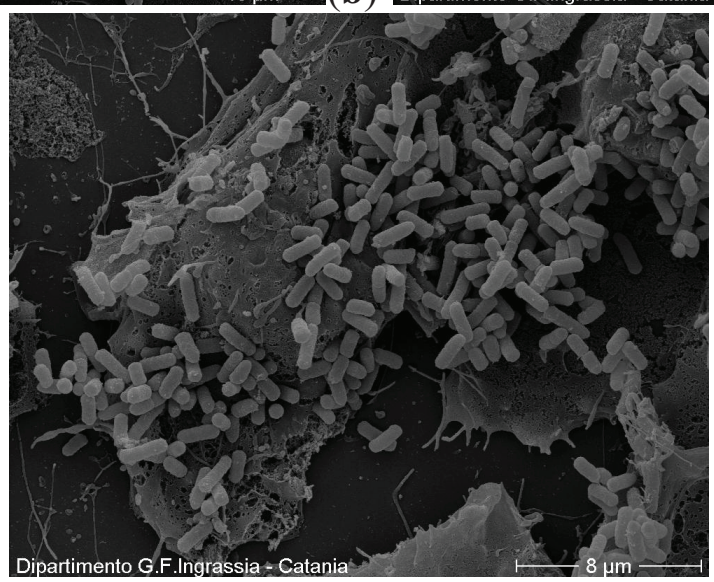

\subsection{Motility Test}

Only for $P$. mirabilis 2 and $P$. mirabilis 4 we observed a reduction of swarming capacity with respect to the control (Figure 6). For the other P. mirabilis strains, substantial differences in the swarming were not observed. The cause of this different behavior is unknown at present but will be the subject of further in-depth analysis.

Figure 6. (a,b) Reduction of swarming capacity observed for two P. mirabilis strains. The lower size of growth halo can be seen in both right plates.

(a)

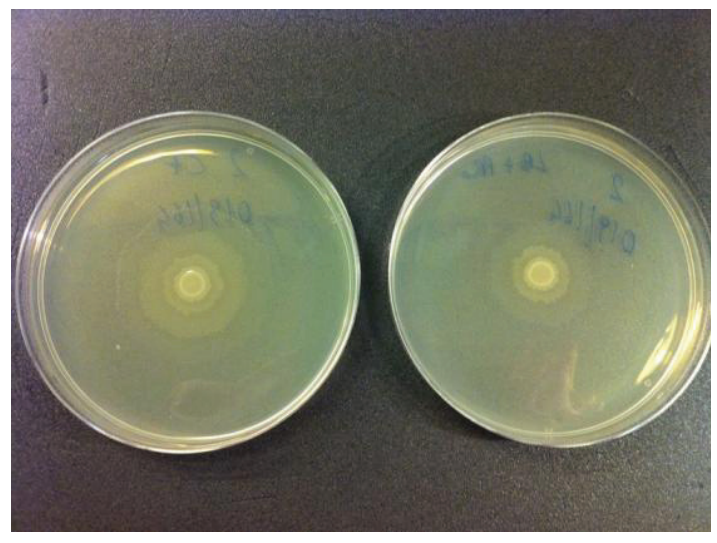


Figure 6. Cont.

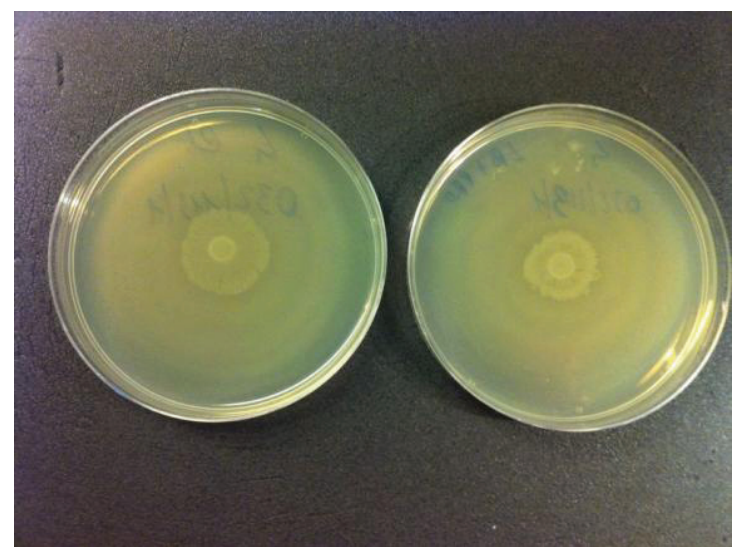

(b)

\subsection{Production of Urease}

The capacity to hydrolyze urea, thanks to an enzyme called urease, producing ammonia and carbon dioxide, is considered one of the principal characteristics of the species of Proteus. This was thus used as an identifying criterion to distinguish them from other non-fermenting bacteria belonging to the family of the Enterobacteriaceae.

In Christensen's medium, Proteus was able to use the urea as the only source of nitrogen, thus producing a dose of ammonia sufficient to change the color of the medium from yellow to fuchsia. The change of color was due to the presence of red phenol, contained in the medium, which is yellow up to $\mathrm{pH} 6.8$ and then becomes a bright red at $\mathrm{pH} 8.2$.

The absorbance mean value for P. mirabilis 3 was 0.075 OD with respect to 0.203 OD of the control. The $\mathrm{pH}$ mean value was 7 vs. 8.5 of the control. P. mirabilis 4 showed the same behavior, with 0.105 as average OD (versus 0.417 of the control) and 7.5 as average $\mathrm{pH}$ (versus 9 of the control) respectively. On the contrary no substantial differences were observed for the remaining two strains examined, neither in absorbance nor in $\mathrm{pH}$. The change of media color, actually, was not evident for both strains, showing a limited urease activity. The causes for the discrepancy in the results observed for the examined strains is still unknown and will be the subject of further analyses.

\subsection{Discussion}

Cranberries have been used for decades for the prevention and treatment of UTIs. Numerous studies have been carried out supporting their efficacy in reducing the number of relapses in women subject to recurrent infections [13-17]. It is well known that their efficacy depends on the anti-adhesive effect of proanthocynidins that they contain on the fimbriae of the uropathogenic strains of E. coli. In this study we wanted to demonstrate a similar effect also on the fimbriae expressed by $P$. mirabilis, as well as a reduction of motility and urease activity, two other important virulence factors.

P. mirabilis can exist in two different morphotypes: in the first it has $6 / 10$ peritrichous flagella that allow individual movement; in the second it has thousands of flagella that allow a particular type of movement, defined swarming. The two different types of movement alternate during the 
growth of Proteus. When the bacterium is of the second morphotype, the cells move a lot then undergo a temporary halt when the bacterium changes to the first morphotype. The result of this alternation is the characteristic growth in concentric circles that can be seen on the agar dishes. Both these types of movement are made by means of the flagella, the filaments composed principally of a protein called flagellin. The protein FlaA is codified by the flaA gene [20] and by flhD. It seems that the activity of this gene, over expressed during the phase of swarming, is notably reduced by exposure to PACs [21].

Also, the urease activity is slightly inhibited. By means of hydrolysis of the urea, the bacteria obtains a source of nitrogen but, contemporarily, this leads to the formation of crystals that can block the catheter or damage kidneys and bladder [22,23]. For this reason, infection from Proteus causes severe damage to tissues with respect to that from E. coli. The activity of cranberries, and of proanthocyanidins in particular, on the species of Proteus has to date been poorly investigated [21]. From the results of this study it seems that they could also play an important role in the prevention of infections caused by this microorganism.

\section{Experimental}

\subsection{Bacterial Strains}

For our experiments we used 5 strains of $P$. mirabilis and 5 of $E$. coli isolated from the urine of women with a history of recurrent cystitis, a standard strain of P. mirabilis (DSM4479) and a standard strain of E. coli (ATCC11775).

\subsection{Adhesion Assay}

The adhesion assay was carried out using the method of Di Martino et al. [24] slightly modified. The HT1376 cells, from human bladder carcinoma, were grown on a sterile cover glass in 24-well plates at $37{ }^{\circ} \mathrm{C}$ in Minimal Essential Medium (MEM) with fetal bovine serum at $10 \%, 2 \mathrm{mM}$ of glutamine and $100 \mu \mathrm{g} / \mathrm{mL}$ of streptomycin. Before infection the cells were washed with PBS to remove any antibiotic present in the culture medium.

The bacteria were grown for $36 \mathrm{~h}$ at $37{ }^{\circ} \mathrm{C}$ in Luria-Bertani broth with the addition of type A proanthocyanidins, at the most efficacious concentration of $50 \mu \mathrm{g} / \mathrm{mL}$ [18] and then at the concentrations of 100, 75, 25 and $15 \mu \mathrm{g} / \mathrm{mL}$. They were then centrifuged and re-suspended in MEM at a concentration of $0.5 \mathrm{McF}$ arland, which corresponds to $10^{8} \mathrm{CFU} / \mathrm{mL}$. Then the prepared bacterial suspensions were placed in contact with the cells $(1 \mathrm{~mL}$ of suspension per well) and incubated at $37^{\circ} \mathrm{C}$ for $3 \mathrm{~h}$. Finally they were washed three times with PBS to remove any bacteria that had not adhered, the cells were then fixed with methanol, colored with Giemsa at $10 \%$ and observed at the microscope. The adhesion index obtained is the average number of adhering bacteria per cell, from an examination of 100 cells. Each test was performed in triplicate.

\subsection{Scanning Electron Microscopy}

Sterile cover glasses similar to those used for adhesion assay were fixed with $3 \%$ glutaraldehyde in $0.12 \mathrm{M}$ phosphate buffer solution $(\mathrm{pH} 7.2)$ at $4{ }^{\circ} \mathrm{C}$ for $1 \mathrm{~h}$. The samples were post-fixed in $1 \%$ 
osmium tetroxide in the same buffer, dehydrated in ethanol, critical point dried and sputter coated with a $5 \mathrm{~nm}$ gold layer using an Emscope SM 300. A Hitachi S-4000 field emission scanning electron microscope was used for the observation [25].

\subsection{Motility Test}

To evaluate the activity of type A PACs on the motility of P. mirabilis, a drop was removed from the 6 suspensions used for the experiment described above and inoculated in the same number of plates containing Mueller-Hinton agar. As control, a further 6 plates were inoculated with a suspension of bacteria grown in Luria-Bertani broth for $36 \mathrm{~h}$ at $37{ }^{\circ} \mathrm{C}$ without the addition of PACs. The plates were then incubated for $24 \mathrm{~h}$ at $37^{\circ} \mathrm{C}$ and the swarming was then observed [26].

\subsection{Production of Urease}

To evaluate the inhibition activity of PACs on the production of urease Christensen's medium was used in the liquid form, with the addition of a solution of urea at $40 \%$. For the inoculum we used the suspensions already prepared for the adhesion assay in which the 5 wild strains of $P$. mirabilis and the collection strain were grown both in the presence and absence of PACs. A drop of each suspension was inoculated in a test tube containing $9.5 \mathrm{~mL}$ of medium and $0.5 \mathrm{~mL}$ of urea and incubated for $3 \mathrm{~h}$ at $37^{\circ} \mathrm{C}$. Obviously in all the test tubes the change in color from yellow to fuchsia was observed. At this point the suspensions were read at the spectrophotometer at $570 \mathrm{~nm}$ to show the difference between the strains used as control and those grown in contact with PACs. The test was repeated three times. Moreover, $\mathrm{PH}$ of all solutions was examined through a $\mathrm{pH}$ Meter [27].

\section{Conclusions}

This work evaluated the efficacy of type A2 PACs, contained in cranberries, in the prevention of UTIs [28]. Our results show that, other than the well-known activity on uropathogenic strains of $E$. coli, they are active also on strains of $P$. mirabilis, reducing the ability to adhere to the epithelial cells of the bladder. These results indicate that PACs also partly inhibit the activity of the flagella, resulting in reduced motility and urease production. Further studies are needed to better characterize the latter features.

\section{Author Contributions}

D. Nicolosi and C. Genovese have been involved in bibliographic research, laboratory tests, data collection and analysis and manuscript drafting.

G. Tempera and P.M. Furneri have been involved in research planning and organization and in checking the draft of the manuscript.

\section{Conflicts of Interest}

The authors declare no conflict of interest. 


\section{References}

1. Foxman, B. Epidemiology of urinary tract infections: Incidence, morbidity, and economic costs. Am. J. Med. 2002, 113, 5-13.

2. Foxman, B. Recurring urinary tract infection: incidence and risk factors. Am. J. Public Health 1990, 80, 331-333.

3. Foxman, B.; Gillespie, B.; Koopman, J.; Zhang, L.; Palin, K.; Tallman, P.; Marsch, J.V.; Spear, S.; Sobel, J.D.; Marty, M.J.; et al. Risk factors for second urinary tract infection among college women. Am. J. Epidemiol. 2000, 151, 1194-1205.

4. Stamm, W.E. Estrogens and urinary tract infection. J. Infect. Dis. 2007, 195, 623-624.

5. Grabe, M.; Bjerklund-Johansen, T.E.; Botto, H.; Çek, M.; Naber, K.G.; Pickard, R.S.; Tenke, P.; Wagenlehner, F.; Wullt, B. Guidelines on Urological Infections. European Association of Urology 2013. Available online: http://www.uroweb.org/gls/pdf/18_Urological\% 20infections_LR.pdf (accessed on 28 March 2014).

6. Lichtenberger, P.; Hooton, T.M. Antimicrobial prophylaxis in women with recurrent urinary tract infections. Int. J. Antimicrob. Agents 2011, 38, 36-41.

7. Rozalski, A.; Sidorczyk, Z.; Kotelko, K. Potential virulence factors of Proteus bacilli. Microbiol. Mol. Biol. Rev. 1997, 61, 65-98.

8. Zunino, P.; Sosa, V.; Schlapp, G.; Allen, A.G.; Preston, A.; Maskell, D.J. Mannose-resistant Proteus-like and P. mirabilis fimbriae have specific and additive roles in P. mirabilis urinary tract infections. FEMS Immunol. Med. Microbiol. 2007, 51, 125-133.

9. Zunino, P.; Sosa, V.; Allen, A.G.; Preston, A.; Schlapp, G.; Maskell, D.J. Proteus mirabilis fimbriae (PMF) are important for both bladder and kidney colonization in mice. Microbiology 2003, 149, 3231-3237.

10. Johnson, D.E.; Russell, R.G.; Lockatell, C.V.; Zulty, J.C.; Warren, J.W.; Mobley, H.L. Contribution of Proteus mirabilis urease to persistence, urolithiasis, and acute pyelonephritis in a mouse model of ascending urinary tract infection. Infect. Immun. 1993, 61, 2748-2754.

11. Rocha, S.P.; Pelayo, J.S.; Elias, W.P. Fimbriae of uropathogenic Proteus mirabilis. FEMS Immunol. Med. Microbiol. 2007, 51, 1-7.

12. Sareneva, T.; Holthofer, H.; Korhonen, T.K. Tissue-binding affinity of Proteus mirabilis fimbriae in the human urinary tract. Infect. Immun. 1990, 58, 3330-3336.

13. Tempera, G.; Corsello, S.; Genovese, C; Caruso, F.E.; Nicolosi, D. Inhibitory activity of cranberry extract on the bacterial adhesiveness in the urine of women: An ex-vivo study. Int. J. Immunopathol. Pharmacol. 2010, 23, 611-618.

14. Wang, C.H.; Fong, C.C.; Chen, N.C.; Liu, S.S.; You, P.H.; Wu, T.Y.; Chen, W.T.; Lee, C.C.; Chen, S.C. Cranberry-containing products for prevention of urinary tract infections in susceptible populations: A systematic review and meta-analysis of randomized controlled trials. Arch. Int. Med. 2012, 172, 988-996.

15. Nowack, R.; Schmitt, W. Cranberry juice for prophylaxis of urinary tract infections - Conclusions from clinical experience and research. Phytomedicine 2008, 15, 653-667.

16. Howell, A.B. Bioactive compounds in cranberries and their role in prevention of urinary tract infections. Mol. Nutr. Food Res. 2007, 51, 732-737. 
17. Raz, R.B.; Chazan, B.; Dan, M. Cranberry juice and urinary tract infection. Clin. Infect. Dis. 2004, 38, 1413-1419.

18. Jepson, R.G.; Craig, J.C. Cranberries for preventing urinary tract infections. Cochrane Database Syst. Rev. 2008, 1, CD001321.

19. Ermel, G.; Georgeault, S.; Inisan, C.; Besnard, M. Inhibition of adhesion of uropathogenic Escherichia coli bacteria to uroepithelial cells by extracts from cranberry. J. Med. Food 2012, 15, 126-134.

20. Manos, J.; Belas, R. The genera Proteus, Providencia, and Morganella. In The Prokaryotes, 3rd ed.; Dworkin, S.F.M., Falkow, S., Rosenberg, E., Schleifer, K.H., Stackebrandt, E., Eds.; Springer: New York, NY, USA, 2006; Volume 6, pp. 245-269.

21. McCall, J.; Hidalgo, G.; Asadishad, B.; Tufenkji, N. Cranberry impairs selected behaviors essential for virulence in Proteus mirabilis HI4320. Can. J. Microbiol. 2013, 59, 430-436.

22. Griffith, D.P.; Musher, D.M.; Itin, C. Urease. The primary cause of infection-induced urinary stones. Investig. Urol. 1976, 13, 346-350.

23. Mobley, H.L.T.; Warren, J.M. Urease-positive bacteriuria and obstruction of long-term urinary catheters. J. Clin. Microbiol. 1987, 25, 2216-2217.

24. Di Martino, P.; Agniel, R.; David, K.; Templer, C.; Gaillard, J.L.; Denys, P.; Botto, H. Reduction of Escherichia coli adherence to uroepithelial bladder cells after consumption of cranberry juice: A double-blind randomized placebo-controlled cross-over trial. World J. Urol. 2006, 24, 21-27.

25. Nicolosi, D.; Scalia, M.; Nicolosi, V.M.; Pignatello, R. Encapsulation in fusogenic liposomes broadens the spectrum of action of vancomycin against Gram-negative bacteria. Int. J. Antimicrob. Agents 2010, 35, 553-558.

26. Muñoz-Criado, S.; Muñoz-Bellido, J.L.; Alonso-Manzanares, M.A.; Gutiérrez-Zufiaurre, M.N.; García-Rodríguez, J.A. Psychotropic drugs inhibit swarming in Proteus spp. and related genera. Clin. Microbiol. Infect. 1998, 4, 447-449.

27. Konieczna, I.; Żarnowiec, P.; Kwinkowski, M.; Kolesińska, B.; Frączyk, J.; Kamiński, Z.; Kaca, W. Bacterial urease and its role in long-lasting human diseases. Curr. Protein Pept. Sci. 2012, 13, 789-806.

28. Foo, L.Y.; Lu, Y.; Howell, A.B.; Vorsa, N. A-Type Proanthocyanidin Trimers from Cranberry that Inhibit Adherence of Uropathogenic P-Fimbriated Escherichia coli. J. Nat. Prod. 2000, $63,1225-1228$. 


\title{
Chapter 3
}

\section{Uncomplicated Urinary Tract Infections and Antibiotic Resistance-Epidemiological and Mechanistic Aspects}

\author{
Bernd Wiedemann, Anke Heisig and Peter Heisig
}

\begin{abstract}
Uncomplicated urinary tract infections are typically monobacterial and are predominantly caused by Escherichia coli. Although several effective treatment options are available, the rates of antibiotic resistance in urinary isolates of $E$. coli have increased during the last decade. Knowledge of the actual local rates of antibiotic resistant pathogens as well as the underlying mechanisms are important factors in addition to the geographical location and the health state of the patient for choosing the most effective antibiotic treatment. Recommended treatment options include trimethoprim alone or in combination with sulfamethoxazol, fluoroquinolones, $\beta$-lactams, fosfomycin-trometamol, and nitrofurantoin. Three basic mechanisms of resistance to all antibiotics are known, i.e., target alteration, reduced drug concentration and inactivation of the drug. These mechanisms-alone or in combination - contribute to resistance against the different antibiotic classes. With increasing prevalence, combinations of resistance mechanisms leading to multiple drug resistant (mdr) pathogens are being detected and have been associated with reduced fitness under in vitro situations. However, mdr clones among clinical isolates such as E. coli sequence type 131 (ST131) have successfully adapted in fitness and growth rate and are rapidly spreading as a worldwide predominating clone of extraintestinal pathogenic E. coli.
\end{abstract}

Reprinted from Antibiotics. Cite as: Wiedemann, B.; Heisig, A.; Heisig, P. Uncomplicated Urinary Tract Infections and Antibiotic Resistance-Epidemiological and Mechanistic Aspects. Antibiotics 2014, 3, 341-352.

\section{Introduction}

Uncomplicated urinary tract infections are among the most common infectious diseases in the community and occur in patients without any anatomic or functional abnormality. About 50\% to $70 \%$ of all women acquire such an infection at least once during their life [1]. Data on the prevalence and antibiotic resistance of the bacteria causing these infections are difficult to obtain, as these infections are treated empirically without bacteriological testing. However, the knowledge of the pathogens and their sensitivities towards the most commonly used antibiotics is essential for a successful treatment and helps avoiding development of resistance [2].

More than $90 \%$ of uncomplicated urinary tract infections are monomicrobial [3]. They are mainly (between $85 \%$ and $90 \%$ ) caused by E. coli, to a lesser extent by other Enterobacteriaceae, Enterococci and Staphylococci [2]. The recent ECO.SENS study [4] reports a frequency of E. coli of $74.2 \%$ in patients from Austria, Greece, Portugal, Sweden, and UK. Similar frequencies for 
E. coli are reported by the ARESC study [5] and the findings by Dong Sup Lee et al. [6]. In addition, these aforementioned studies report 3.4\% and 2.3\% P. mirabilis, $4.1 \%$ and $5.6 \%$ Enterococci, $3.5 \%$ and $4.7 \%$ K. pneumoniae, $1.1 \%$ and $2.3 \%$ Enterobacter spp., other bacteria were found with $11.2 \%$ and $7 \%$, respectively. However, these data are derived from designed studies which have to be interpreted with caution: First, usually no microbiological testing is performed for patients suffering from uncomplicated urinary tract infections. Second, it is difficult to obtain reliable local data on the incidence of resistant strains from respective patients. Third, different surveillance systems do neither use the identical methodology to measure susceptibility nor the identical breakpoints for classifying resistant and sensitive bacteria. Beside laboratory data on the in vitro susceptibility of the presumptive causative agent, the application of antibiotic stewardship, which uses data not only on the local and global epidemiological situation of antibiotic resistance, but also the potential impact of antibiotics on the microflora of the patient, provides a rationale for choosing an appropriate antibiotic for treatment. The aim of this review is to provide an overview of epidemiology and mechanisms of resistance for antibiotics frequently used in the treatment of uncomplicated urinary tract infections.

\section{Therapeutic Options}

Besides pharmacodynamic, pharmacokinetic, and tolerability aspects developing or already existing resistance to the drug to be chosen for treatment is the most important motivation for selecting an antibiotic. Furthermore, the activity of the drug against the resident bowel flora, as well as the effect of the duration of the treatment on the probability to develop resistant bacteria may have an important impact [1].

Considering the afore mentioned factors, therapeutic options for the treatment of uncomplicated, community acquired urinary tract infections have been developed, based on few long experienced antibiotics for oral application. The treatment of acute uncomplicated cystitis as recommended by the guidelines of the European Association of Urology (EAU) [7] includes fosfomycin-tromethamol, pivmecillinam, and nitrofurantoin as first line therapy. As an alternative therapy, fluoroquinolones, cefpodoxime proxetil, cotrimoxazole and trimethoprim are possible options, if the local resistance rate is less than $20 \%$. These recommendations should be adjusted taking into account the geographical location of the patient, age, and sex as well as other diseases. A similar restriction is valid for $\beta$-lactam antibiotics like amoxicillin, amoxicillin/clavulanic acid and pivmecillinam, which are used in some countries. The recommended duration of treatment takes into account the necessary time for effectiveness and the risk of resistance development as a result of prolonged selective pressure. Usually, the duration of treatment is three days. Fosfomycin is given even in a single dose, amoxicillin and nitrofurantoin, however, require five to seven-day treatment [8].

\section{Antibiotic Resistance-Genetic and Mechanistic Basis}

The development of antibiotic resistant mutants from susceptible cells is driven by two characteristic features of prokaryotic cells — high growth rate and a haploid genome: The rapid growth rate yields a large population size within a short period of time and this increases the probability to yield one mutant cell within typically $10^{8}$ cells due to the fortuitous acquisition of a resistance mutation [9]. 
Slow growing bacteria, like Mycobacterium tuberculosis overcome their growth deficiency by expression of immune evasion mechanisms which ensure the undisturbed local persistence of populations over periods of time long enough to develop resistant mutants [10].

The haploid genome structure allows for an immediate expression of a mutant genotype in bacteria. Beside mutations, which alter existing genetic material resulting in antibiotic resistance, another genetic strategy to acquire antibiotic resistance is the transfer of genetic material encoding antibiotic resistance between cells of mixed bacterial populations by either transformation of naked DNA, conjugation of plasmid DNA via cell-cell-contact, or phage-mediated transduction [11]. Either genetic alteration can result in one of three basic biochemical mechanisms of resistance: a reduction of the affinity of the target for an antibiotic, a reduction of the concentration of the drug at the target site, and an enzymatic inactivation of the drug. During evolution under selective pressure, bacterial cells have developed numerous variations of these three basic mechanisms which alone or in combination can result in clinically relevant resistance to specific or even all known antibiotics [12]. However, the acquisition of antibiotic resistance can be associated with a reduced fitness/virulence of the resistant cell due to an impairment of the normal function of the affected target or to the overexpression of a specific resistance gene [13,14]. While in the presence of the selecting antibiotic the resistant mutant has a growth advantage, in the absence of the antibiotic, i.e., after the therapy is completed, reduced fitness can turn this into a disadvantage. Over time, antibiotic is resistant, but less fit mutants can acquire additional genetic alterations which enable them to compensate for the fitness reduction. Finally, the combination of resistance and compensatory mutations can give rise to well adapted clones capable of spreading among host populations.

\section{Resistance to Sulfonamides and Trimethoprim-Epidemiology and Mechanisms}

The use of sulfonamides alone or in combination with the dihydropyrimidine derivative trimethoprim has a long history in the treatment of uncomplicated urinary tract infections. Since these infections are often treated empirically without susceptibility testing only a few data on resistance of the pathogen are available. Percentages of E. coli strains resistant to cotrimoxazole vary with the geographical location of the patients: $35.9 \%$ in Korea [6], 25.4\% [15], 16.1\% [4], and $12.2 \%$ [16] in Europe. According to recent data from Kahlmeter et al. [4] the percentages are 15.9, 18.2, 16.7, 16.3, and 14.4 for Austria, Greece, Portugal, Sweden and UK, respectively. These authors also described changing incidences from the first study in 1999-2000 to the second study in 2008: Portugal showed a drop from $26.7 \%$ to $16.7 \%$ and Sweden an increase from $8.3 \%$ to $16.3 \%$ resistant strains. Data for sulfonamides and trimethoprim alone are scarce, $24.8 \%$ and $16.7 \%$, respectively [6].

Sulfonamides such as sulfamethoxazol (SMX) and dihydropyrimidines such as trimethoprim (TMP) target dihydropteroate synthetase (DHPS, the sul gene product) and dihydrofolate reductase (DHFR, the $d f r$ gene product), respectively. Both enzymes catalyze either of two subsequent steps in the bacterial biosynthesis of folic acid [17]. Recent crystallographic data revealed that a conserved binding pocket of DHPS for the natural substrate para-aminobenzoic acid ( $\mathrm{p}-\mathrm{ABA}$ ) is formed only in an intermediate reaction step during the catalytic cycle. The resulting covalent bond formed between p-ABA and a pteridin cation yields 7,8-dihydropteroate [18]. In an analogous mode of 
action involving dynamic conformational changes of DHFR from a closed to an occluded state, this enzyme catalyzes the reduction of the substrate dihydrofolate to tetrahydrofolate (THF) by using nicotinamide adenine dinucleotide phosphate (NADPH) as a cofactor [19]. Inhibition of either enzyme by sulfonamide or trimethoprim causes a shortage of folic acid, which is an essential cofactor also for the biosynthesis of purine nucleotides, thymine, nucleic acids, and serine. As a consequence DNA replication stops and this event finally causes cell death.

Resistance to SMX and TMP could easily be selected in vitro from susceptible strains of E. coli. Resulting mutants were demonstrated to have acquired mutational alterations of the chromosomal $s u l$ and $d f r$ genes. Such modifications have also been identified as a cause of primary resistance to sulfonamide in chromosomal sul genes of those species which are naturally competent pathogens such as Streptococcus pneumoniae, Campylobacter jejuni, Neisseria meningitidis, and Neisseria gonorrhoeae, capable of taking up from the environment foreign DNA fragments released from dead cells, and subsequently integrating them into their chromosome. However, comparison of sul and $d f r$ gene sequences from isolates belonging to the above mentioned species, suggest a horizontal transfer of antibiotic resistant gene copies and subsequent integration of resistance-determining gene sequences from the acquired gene into homologous regions of the chromosomal copy. The resulting sequences form so-called mosaic genes carrying a central antibiotic resistant gene fragment of acquired DNA flanked by regions of the residing chromosomal gene copy [20].

In contrast, the most frequent mechanism of resistance in clinical isolates of $E$. coli and other enterobacteria from urinary tract infections is the acquisition of resistant variants of complete sul and $d f r$ genes expressing enzyme variants which are refractory to the inhibitory activity of the respective drug at clinically achievable concentrations. More than 30 resistant variants of trimethoprim resistant $d f r$ genes and three variants of sulfonamide resistant sul genes have been described so far [20]. Many of these are encoded by mobile genetic elements residing on transferable plasmids in combination with other resistance genes. As a consequence, multiple resistance genes are cotransferred en bloc. An unusual genetic constellation has been detected in sulfonamide resistant $E$. coli isolates belonging to clonal group A, which have been isolated from different US regions. All isolates carry a genomic resistance module consisting of several resistance genes integrated in a specific chromosomal locus [21].

\section{Resistance to Fluoroquinolones-Epidemiology and Mechanisms}

Resistance to fluoroquinolones in E. coli is quite high in many European countries ranging from $25 \%$ to $50 \%$ [22]. The prevalence of resistant strains, according to few surveillance data available on uncomplicated urinary tract infections, was reported to be much lower, i.e., $28.2 \%, 13.9 \%, 3.9 \%$, and $1.0 \%$ according to references [4,6,15,16], respectively. However, again Kahlmeter, 2012 [4] reports an increase from 2000 to 2008 in most countries in Austria ( $0 \%$ to $4.1 \%)$, in Greece (1.5\% to $5.7 \%$ ), in Portugal (5.8\% to 7.6\%), in Sweden ( $0 \%$ to $2.5 \%$ ), and in the United Kingdom ( $0.5 \%$ to $0.6 \%$ ). The incidence of resistant strains isolated from blood in 2008 was $22.9 \%$ in Austria, $22.4 \%$ in Greece, $28.6 \%$ in Portugal, $10.3 \%$ in Sweden, and 15.1\% in the United Kingdom according to EARS Net. The source of infection in uncomplicated UTIs is the bowel flora of the patient or the sexual partner. The resistance is much lower in relation to surveillance data from hospitals. 
Fluoroquinolones were first introduced into clinical use in 1985. The high clinical efficacy of orally available fluoroquinolones norfloxacin, ofloxacin and ciprofloxacin together with the initially very low incidence of resistance in E. coli and many other Gram-negative pathogens in the treatment of urinary tract infections rapidly resulted in a widespread empirical use for this application. This high efficacy is due to the high affinity and inhibitory activity of the drugs to their target topoisomerases gyrase and topoisomerase IV which are $A_{2} B_{2}$ tetrameric enzymes sharing high structural and functional homology. Consequences of the irreversible enzyme inhibition are the arrest of replicative DNA metabolism and subsequent cell death due to secondary bactericidal mechanisms such as the introduction of DNA double strand breaks following the inhibition of DNA gyrase [23,24].

The development of clinically relevant resistance to fluoroquinolones in E. coli has been investigated intensively and has been demonstrated to require multiple mutation steps which involve alterations in conserved regions of both chromosomally encoded target gene pairs gyrA/parC and gyrB/parE encoding subunits A and B of topoisomerase II/IV, respectively. These result in a reduced affinity of the drugs to the mutated target [25]. In addition, a reduced drug concentration at the target site had been associated with chromosomal mutations either reducing the amount of outer membrane porin OmpF, a water-filled transmembrane channel which allows water-soluble small molecules such as fluoroquinolones to enter the cell, or by an increased expression of multiple drug-resistance (MDR) efflux pump AcrAB-TolC which actively exports antibiotics of different classes out of the cell, or by a combination of both. The latter mechanism is due to genetic alterations inactivating chromosomally encoded local (AcrR) or global negative regulators (MarR, SoxR, and RamR) which control the expression of MDR efflux pump AcrAB-TolC. Global regulators have been demonstrated to simultaneously control the expression of major porin OmpF via an antisense RNA switch.

Besides these mechanisms, several non-target based mechanisms of plasmid-mediated quinolone resistance (PMQR) have been detected during the last decade. A PMQR mechanism alone mediates only a low-level fluoroquinolone resistance resulting in MIC increases below the breakpoint. PMQR are subdivided into (I) mechanisms associated with reduced drug concentration at the target site due to the expression of plasmid-encoded quinolone efflux pumps QepA or OqxAB; (II) mechanisms protecting the target site, as has been determined for the different Qnr proteins belonging to either of the pentapeptid repeat protein families QnrA, QnrB, QnrC, QnrD, or QnrS; and (III) a mechanism exclusively inactivating C7-piperazinyl substituted fluoroquinolones norfloxacin and ciprofloxacin by an acetyltransferase mechanism. This unique enzyme is derived from an aminoglycoside-modifying acetyltransferase AAC6'(Ib) by the acquisition of two point mutations which extend the substrate spectrum to two different antibiotic classes [26].

While mechanisms of PMQR are reported with increasing prevalence also in E. coli isolates mediating UTI in humans and animals, their impact on clinically relevant resistance is lower compared to mutations affecting target topoisomerases gyrase and topoisomerase IV, but in combination PMQR contribute to an increase in the resistance level [27]. In addition, a possible role of PMQR as pacemakers of the development of clinical resistance to fluoroquinolones is being discussed. This view is supported by in vitro studies demonstrating an impact of $q n r$ genes in E. coli isolates from UTI on fluoroquinolone activity in an in vitro model [28] as well as in a mouse infection 
model $[29,30]$. Former use of fluoroquinolones has been identified as a relevant risk factor for the development of clinically relevant resistance to these drugs [31].

\section{Mechanisms of Resistance to $\beta$-Lactam Antibiotics-Epidemiology and Mechanisms}

For uUTI treatment pivmecillinam (PIV), the prodrug of the active compound mecillinam, is recommended as first-line drug in several countries [1]. PIV shows good clinical cure rates against Gram-negative pathogens including E. coli expressing extended-spectrum $\beta$-lactamases (ESBL) such as ST131 isolates encoding CTX-M14, CTX-M15 [32]. Therefore, resistance to cefotaxime or ceftazidime can be used as a good marker for ESBL prevalence. Such data obtained from EcoSens study II revealed increasing but still low prevalence of ESBL producing E. coli from uUTI in Europe [4]. Due to high concentrations of $\beta$-lactam drugs in the bowel and a relatively long treatment period all $\beta$-lactams exert a high degree of selection pressure. Prevalence of resistant $E$. coli strains for amoxicillin is far above $20 \%$, so that this drug should not be used for treatment of these infections at all. The addition of clavulanic acid restores susceptibility for most strains. Data from Kahlmeter [4] demonstrate that the percentage of amoxicillin/clavulanic acid-resistant $E$. coli strains differs from country to country: Austria, 8.9\%, Greece 4.3\%, Portugal 6.9\%, Sweden 2.5\%, and United Kingdom 2.0\%. Data from other studies also show a varying realation of $E$. coli isolates from uncomplicated urinary tract infections resistant to amoxicillin/resistant to amoxicillin plus clavulanic acid, such as $63.6 \% / 5.5 \%$ [6], 42.4/7.5 [15], 28.0/4.5 [4], and 35.5/1.5 [16].

The predominant mechanism of resistance to $\beta$-lactam antibiotics in E. coli and most other Gram-negatives is the production of a $\beta$-lactamase which enzymatically inactivates $\beta$-lactam antibiotics by hydrolysis of the essential $\beta$-lactam ring. As a consequence the $\beta$-lactam will not more bind to its targets, the cell wall synthesizing transpeptidases/transglycosylases also designated as penicillin binding proteins (PBPs). According to the active site architecture $\beta$-lactamases either belong to the serine protease type or to the metallo enzyme type. While the catalytic site of a serine protease is formed by a conserved triad of amino acids aspartate, histidine and serine [33], that of a metallo enzyme is composed of a central catalytically active $\mathrm{Zn}^{2+}$ ion chelated by a set of four conserved histidin and/or cystein amino acids [34]. While some enterobacterial genera encode a chromosomal $\beta$-lactamase active against a broad spectrum of $\beta$-lactam antibiotics whose expression can be induced by $\beta$-lactams, the clinically most relevant enzymes are plasmid-encoded extended spectrum $\beta$-lactamases (ESBL), serine $\beta$-lactamases, which hydrolyze most $\beta$-lactams with the exception of carbapenems, but can be inhibited by $\beta$-lactamase inhibitors such as clavulanic acid [35].

Many ESBL enzymes belonging to class A are grouped into one of three major families: TEM, SHV and CTX-M. Within each family a high degree of DNA and amino acid sequence homology is found. Individual family members differ from their parent enzyme mediating only broad-spectrum activity by a few point mutations. These mutations affect regions associated either with the access of the drug to the binding pocket containing the active-site serine or with the kinetic properties of the enzyme resulting in an acceleration of drug inactivation. The resulting enzymes mediate resistance to extended spectrum $\beta$-lactams which includes resistance to cefotaxime and ceftazidime as a good marker for an ESBL phenotype [36]. Beside these "classical" ESBL enzymes, several new derivatives of classes $C$ and $D \beta$-lactamases have evolved which share characteristics of an ESBL phenotype [37]. Despite of an increasing prevalence of ESBL-producing E. coli in UTI the clinical 
efficacy

$\beta$-lactams in the treatment of acute uncomplicated urinaryt tract infections is often less affected by this resistance mechanism presumably due to the high drug concentrations in urine [32]. E. coli strains belonging to the epidemiologically dominating sequence type 131 (ST131) express a multiple drug resistance phenotype which includes sulfonamide/trimethoprime and fluoroquinolones in addition to $\beta$-lactams due to the acquisition of a plasmid carrying a bla $a_{C T X-M-15}$-encoding ESBL gene [37].

\section{Resistance to Fosfomycin-Epidemiology and Mechanisms}

Fosfomycin is rarely used in clinical settings, it is used only in combination with other powerful drugs like third generation cephalosporins, carbapenems, or aminoglycosides in severe infections, because the mutation rate to generate resistant mutants is extremely high. For uncomplicated urinary tract infections, however, the monobasic watersoluble fosfomycin salt, fosfomycin trometamol, is specially designed. After oral application, fosfomycin achieves a urinary concentration, which does not allow the resistant mutants to grow. Therefore, it can be used in this indication as a single drug.

Until now, resistance rates to fosfomycin are low: $1.2 \%$ and $0.2 \%$ acording to [4] and [16], respectively. Kahlmeter [4] found a low prevalence of fosfomycin resistance for all European countries involved, such as Austria $0.7 \%$, Greece 2.9\%, Portugal 0.7\%, Sweden 1.5\%, and United Kingdom $1.5 \%$. A systematic review of clinical studies on the incidence of fosfomycin resistance in clinical isolates from complicated and uncomplicated urinary tract infections shows less than $8 \%$ resistance overall [38].

Fosfomycin is a potent inhibitor of the murA gene product, UDP-N-acetylglucosamine enoylpyruvyl transferase, which catalyzes an essential step in the synthesis of UDP- $N$-acetylmuramic acid, one essential building block for peptidoglycan synthesis. Inhibition of MurA results in an effective stop of the biosynthesis of the bacterial cell wall. However, to inhibit the intracellular target structure MurA, fosfomycin has to pass the cell membrane. This is achieved in E. coli by an active uptake process involving either one of two fosfomycin influx transporters, gylcerol-3-phosphate transporter (the $g l p T$ gene product) and hexose phosphate transporter (the $u h p T$ gene product) [39]. Different mechanisms of resistance to fosfomycin have been identified from in vitro studies. These mechanisms include in $E$. coli a point mutation in murA changing cys-115 into asp thereby preventing the covalent binding of fosfomycin to its target [40]. Variations at this site have been detected in species such as M. tuberculosis, Chlamydia trachomatis, and Vibrio fisheri which are naturally less susceptible to fosfomycin. Another mechanism involves mutations in genes $u h p T$ and glpT which results in reduced drug uptake [39]. However, these chromosomal mutations selected in vitro are associated with reduced fitness. This seems to provide a plausible explanation for the observed low incidence of such mutants in clinical isolates from urinary tract infections in comparison with the relatively frequent isolation of resistant mutants in vitro [41]. However, recent data report the occurrence of clinical isolates in Asia with transferable resistance to fosfomycin. The underlying mechanism is the expression of a glutathione-S-transferase activity which inactivates fosfomycin. Several genes encoding such activity have been identified on resistance plasmids frequently associated with a gene encoding a CTX-M-type $\beta$-lactamase $[42,43]$. 


\section{Resistance to Nitrofurantoin-Epidemiology and Mechanisms}

Nitrofurantoin is the class representative of the nitroimidazoles. After intracellular activation by bacterial nitroreductases it shows excellent bactericidal activity against $E$. coli and a still good, but lesser activity against many other enterobacterial pathogens. This advantage of an as yet extraordinarily low incidence of resistance $(<3 \%)$ is partially outcompeted by an increased risk for severe side effects, such as toxicity for lung and liver [44].

\section{Epidemiological Aspects of Multiple Drug Resistance in Urinary Tract Infections}

During the last decade the isolation of ESBL-producing E. coli isolates belonging to the $\mathrm{O} 25 \mathrm{~b}: \mathrm{H} 4$ serotype from urinary tract infections has increasingly been reported from over the world. Detailed molecular research revealed that these isolates predominantly belong to ST131 and the pathotype B2. Remarkable is the high incidence of fluoroquinolone resistance due to chromosomal mutations in both target topoisomerases [45] as well as the presence of a conjugative plasmid typically carrying a CTX-M-type $\beta$-lactamase such as $-15,-1$, and $-14[46,47]$. A genome analysis of a set of ST131 isolates provided further evidence for a single clone which has spread among patients suffering from community acquired urinary tract infections within a large UK region and has split into a few molecular subgroups presumably due to the acquisition of resistance plasmids mediating varying patterns of antibiotic resistance including $\beta$-lactams, trimethoprim/sulfonamide, tetracycline, or gentamicin [48]. Besides a resistance profile the ST131 cells seem to express specific virulence traits allowing them to successfully spread among patients not only in hospitals, but also in nursing-homes. The detection of ST131 cells in animals varies between different studies suggesting that animals do not play a significant role as reservoirs for human infections. Although local epidemiological data from the Indian subcontinent are limited [49], travel to Pakistan and India is suspected to be a potential risk factor for the acquisition of multiple drug resistant E. coli ST131 [47]. Thus, the epidemiological survey of this pandemic clone requires special attention in the future and may have an impact on the empirical treatment of uncomplicated urinary tract infections.

\section{Acknowledgment}

The authors wish to express thanks to Kurt G. Naber for stimulating discussion during the preparation of the manuscript.

\section{Author Contributions}

B.W. wrote the clinical and epidemiological sections. A.H. and P.H. contributed equally to the molecular mechanisms of resistance sections.

\section{Conflicts of Interest}

The authors declare no conflict of interest. 


\section{References}

1. Hooton, T.C. Uncomplicated urinary tract infection. N. Engl. J. Med. 2012, 366, 1028-1037.

2. Nicolle, L.; Anderson, P.A.M.; Conly, J.; Mainprize, T.C.; Meuser, J.; Nickel, J.C.; Senikas, V.M.; Zhanel, G.G. Uncomplicated urinary tract infection in women. Can. Fam. Phys. 2006, 52, 612-618.

3. Stuck, A.K.; Täuber, M.G.; Schabel, M.; Lehmann, T.; Suter, H.; Mühlemann, K. Determinants of quinolone versus trimethoprim-sulfamethoxazole use for outpatient urinary tract infection. Antimicrob. Agents Chemother. 2012, 56, 1359-1363.

4. Kahlmeter, G.; Odén Poulsen, H. Antimicrobial susceptibility of Escherichia coli from community-acquired urinary tract infections in Europe: The ECO-SENS study revisited. Int. J. Antimicrob. Agents 2012, 39, 45-51.

5. Schito, G.C.; Naber, K.G.; Botto, H.; Palou, J.; Mazzei, T.; Gualco, L.; Marchese, A. The ARESC study: An international survey on the antimicrobial resistance of pathogens involved in uncomplicated urinary tract infections. Int. J. Antimicrob. Agents 2009, 34, 407-413.

6. Lee, D.S.; Choe, H.S.; Lee, S.J.; Bae, W.J.; Cho, H.J.; Yoon, B.I.; Cho, Y.H.; Han, C.H.; Jang, H.; Park, S.B.; et al. Antimicrobial susceptibility pattern and epidemiology of female urinary tract infections in South Korea, 2010-2011. Antimicrob. Agents Chemother. 2013, 57, 5384-5393.

7. Grabe, M.; Bjerklound-Johansen, T.E.; Bartoletti, R.; Wulf, B.; Cek, M.; Naber, K.G.; Pickard, R.S.; Tenke, P.; Wagenlehner, F.; Wullt, B. Guidelines for Urological Infections; European Association of Urology: Arnhem, The Netherlands, 2014.

8. S3-Leitlinie AWMF-Register Nr. 043/044 (Deutsche Gesellschaft für Urologie (DGU), Deutsche Gesellschaft für Allgemeine und Familienmedizin (DEGAM), Deutsche Gesellschaft für Gynäkologie und Geburtsthilfe (DGGG), Deutsche Gesellschaft für Hygiene und Mikrobiologie (DGHM), Deutsche Gesellschaft für Infektiologie (DGI), Deutsche Gesellschft für Nephrologie (DGfN), Paul-Ehrlich-Gesellschaft für Chemotherapie (PEG). Available online: http://www.awmf.org/uploads/tx_szleitlinien/043-0441_S3_Harnwegsinfektionen.pdf (accessed on 18 July 2014).

9. Matic, I.; Radman, M.; Taddei, F.; Picard, B.; Doit, C.; Bingen, E.; Denamur, E.; Elion, J. Highly variable mutation rates in commensal and pathogenic Escherichia coli. Science 1997, 277, 1833-1834.

10. Gupta, A.; Kaul, A.; Tsolaki, A.G.; Kishore, U.; Bhakta, S. Mycobacterium tuberculosis: Immune evasion, latency and reactivation. Immunobiology 2012, 217, 363-374.

11. Davies, J.; Davies, D. Origins and evolution of antibiotic resistance. Microbiol. Mol. Biol. Rev. 2010, 74, 417-433.

12. Yoneyama, H.; Katsumata, R. Antibiotic resistance in bacteria and its future for novel antibiotic development. Biosci. Biotechnol. Biochem. 2006, 70, 1060-1075.

13. Moreno, E.; Prats, G.; Sabate, M.; Perez, T.; Johnson, J.R.; Andreu, A. Quinolone, fluoroquinolone and trimethoprim/sulfamethoxazole resistance in relation to virulence determinants and phylogenetic background among uropathogenic Escherichia coli. J. Antimicrob. Chemother. 2006, 57, 204-211. 
14. Starcic, E.M.; Rijavec, M.; Krizan-Hergouth, V.; Fruth, A.; Zgur-Bertok, D. Chloramphenicol- and tetracycline-resistant uropathogenic Escherichia coli (UPEC) exhibit reduced virulence potential. Int. J. Antimicrob. Agents 2007, 30, 436-442.

15. Linhares, I.; Raposo, T.; Rodrigues, A.; Almeida, A. Frequency and antimicrobial resistance patterns of bacteria implicated in community urinary tract infections: A ten-year surveillance study (2000-2009)._BMC Infect. Dis. 2013, 13, e19.

16. Neuzillet, Y.; Naber, K.G.; Schito, G.; Gualco, L.; Botto, H. French results of the ARESC study: Clinical aspects and epidemiology of antimicrobial resistance in female patients with cystitis. Implications for empiric therapy. Med. Mal. Infect. 2012, 42, 66-75.

17. Sköld, O. Resistance to trimethoprim and sulfonamides. Vet. Res. 2001, 32, 261-273.

18. Yun, M.K.; Wu, Y.; Li, Z.; Zhao, Y.; Waddell, M.B.; Ferreira, A.M.; Lee, R.E.; Bashford, D.; White, S.W. Catalysis and sulfa drug resistance in dihydropteroate synthase. Science 2012, 335, 1110-1114.

19. Bhabha, G.; Lee, J.; Ekiert, D.C.; Gam, J.; Wilson, I.A.; Dyson, H.J.; Benkovic, S.J.; Wright, P.E. A dynamic knockout reveals that conformational fluctuations influence the chemical step of enzyme catalysis. Science 2011, 332, 234-238.

20. Sköld, O. Sulfonamides and trimethoprim. Exp. Rev. Anti-Infect. Ther. 2010, 8, 1-6.

21. Lescat, M.; Calteau, A.; Hoede, C.; Barbe, V.; Touchon, M.; Rocha, E.; Tenaillon, O.; Médigue, C.; Johnson, J.R.; Denamur, E. A module located at a chromosomal integration hot spot is responsible for the multidrug resistance of a reference strain from Escherichia coli clonal group A. Antimicrob. Agents Chemother. 2009, 53, 2283-2288.

22. EARS-Net. Available online: http://www.ecdc.europa.eu/en/healthtopics/antimicrobial_ resistance/database (accessed on 18 July 2014).

23. Heisig, P. Type II topoisomerases-Inhibitors, repair mechanisms and mutations. Mutagenesis 2009, 24, 465-469.

24. Pohlhaus, J.R.; Kreuzer, K.N. Norfloxacin-induced DNA-gyrase cleavage complexes block Escherichia coli replication forks, causing double-stranded breaks in vivo. Mol. Microbiol. 2005, 56, 1416-1429.

25. Heisig, P. Inhibitors of bacterial topoisomerases: Mechanisms of action and resistance and clinical aspects. Planta Med. 2001, 67, 3-12.

26. Strahilevitz, J.; Jacoby, G.A.; Hooper, D.C. Plasmid-mediated quinolone resistance: A multifaceted threat. Clin. Microbiol. Rev. 2009, 22, 664-689.

27. Emrich, N.C.; Heisig, A.; Stubbings, W.; Labischinski, H.; Heisig, P. Antibacterial activity of finafloxacin under different $\mathrm{pH}$ conditions against isogenic strains of Escherichia coli expressing combinations of defined mechanisms of fluoroquinolone resistance. J. Antimicrob. Chemother. 2010, 65, 2530-2533.

28. Cesaro, A.; Roth Dit Bettoni, R.; Lascols, C.; Merens, A.; Soussy, C.J.; Cambau, E. Low selection of topoisomerase mutants from strains of Escherichia coli harbouring plasmid-borne qnr genes. J. Antimicrob. Chemother. 2008, 61, 1007-1015. 
29. Allou, N.; Cambau, E.; Massias, L.; Chau, F.; Fantin, B. Impact of low-level resistance to fluoroquinolones due to qnrAl and qnrSl genes or a gyrA mutation on ciprofloxacin bactericidal activity in a murine model of Escherichia coli urinary tract infection. Antimicrob. Agents Chemother. 2009, 53, 4292-4297.

30. Jakobsen, L.V.; Cattoir, K.S.; Jensen, A.M.; Hammerun, P.; Nordmann, N.; Frimodt-Moller, N. Impact of low-level fluoroquinolone resistance genes qnrA1, qnrB19 and qnrS1 on ciprofloxacin treatment of isogenic Escherichia coli strains in a murine urinary tract infection model. J. Antimicrob. Chemother. 2012, 67, 2438-2444.

31. Christiansen, N.; Nielsen, L.; Jakobsen, L.; Stegger, M.; Hansen, L.H.; Frimodt-Møller, N. Fluoroquinolone resistance mechanisms in urinary tract pathogenic Escherichia coli isolated during rapidly increasing fluoroquinolone consumption in a low-use country. Microb. Drug Resist. 2011, 17, 395-406.

32. Jansaker, F.; Frimodt-Møller, N.; Sjögren, I.; Knudsen, J.D. Clinical and bacterial effects of pivmecillinam for ESBL-producing Escherichia coli or Klebsiella pneumoniae in urinary tract infection. J. Antimicrob. Chemother. 2014, 69, 769-772.

33. Bush, K.; Jacoby, G. Updated functional classification of $\beta$-lactamases. Antimicrob. Agents Chemother. 2010, 54, 969-976.

34. Palzkill, T. Metallo- $\beta$-lactamase structure and function. Ann. N. Y. Acad. Sci. 2013, 1277, 91-104.

35. Paterson, D.L.; Bonomo, R.A. Extended-spectrum beta-lactamases: A clinical update. Clin. Microbiol. Rev. 2005, 18, 657-686.

36. Bush, K.; Fisher, J.F. Epidemiological expansion, structural studies, and clinical challenges of new $\beta$-lactamases from Gram-negative bacteria. Ann. Rev. Microbiol. 2011, 65, 455-478.

37. Cagnacci, S.; Gualco, L.; Debbia, E.; Schito, G.C.; Marchese, A. European emergence of ciprofloxacin-resistant Escherichia coli clonal groups O25:H4-ST1131 and O15:K52:H1 causing community-acquired uncomplicated cystitis. Antimicrob. Agents Chemother. 2008, 46, 2605-2612.

38. Falagas, M.E.; Kastoris, A.C.; Kapaskelis, A.M.; Karageorgopoulo, D.E. Fosfomycin for the treatment of multidrug-resistant including extended-spectrum $\beta$-lactamase producing Enterobacteriaceae infections: A systematic review. Lancet Infect. Dis. 2010, 10, 43-50.

39. Takahata, S.; Ida, T.; Hiraishi, T.; Sakakibara, S.; Maebashi, K.; Terada, S.; Muratani, T.; Matsumoto, T.; Nakahama, C.; Tomono, K. Molecular mechanisms of fosfomycin resistance in clinical isolates of Escherichia coli. Int. J. Antimicrob. Agents 2010, 35, 333-337.

40. Kim, D.H.; Lees, W.J.; Kempsell, K.E.; Lane, W.S.; Duncan, K.; Walsh, C.T. Characterization of a Cys1 15 to Asp substitution in the Escherichia coli cell wall biosynthetic enzyme UDP-GlcNAc enolpyruvyl transferase (MurA) that confers resistance to the antibiotic fosfomycin. Biochemistry 1996, 35, 4923-4928.

41. Karageorgopoulos, D.E.; Wang, R.; Yu, X.H.; Falagas, M.E. Fosfomycin: Evaluation of the published evidence on the emergence of antimicrobial resistance in Gram-negative pathogens. J. Antimicrob. Chemother. 2012, 67, 255-268. 
42. Wachino, J.; Yamane, K.; Suzuki, S.; Kimura, K.; Arakawa, Y. Prevalence of fosfomycin resistance among CTX-M-producing Escherichia coli clinical isolates in Japan and identification of novel plasmid-mediated fosfomycin-modifying enzymes. Antimicrob. Agents Chemother. 2010, 54, 3061-3064.

43. Sato, N.; Kawamura, K.; Nakane, K.; Wachino, J.I.; Arakawa, Y. First detection of fosfomycin resistance gene fos 33 in CTX-M-producing Escherichia coli isolates from healthy individuals in Japan. Microb. Drug Resist. 2013, 19, 477-482.

44. Koulaouzidis, A.; Bhat, S.; Moschos, J.; Tan, C.; de Ramon, A. Nitrofurantoin-induced lungand hepatotoxicity. Ann. Hepatol. 2007, 6, 119-121.

45. Platell, J.L.; Cobbold, R.N.; Johnson, J.R.; Heisig, A.; Heisig, P.; Clabots, C.; Kusowski, M.A.; Trott, D.J. Commonality between fluoroquinolone-resistant sequence type ST131 extraintestinal Escherichia coli isolates from humans and companion animals in Australia. Antimicrob. Agents Chemother. 2011, 55, 3782-3787.

46. Peirano, G.; Pitout, D.D. Molecular epidemiology of Escherichia coli producing CTX-M $\beta$-lactamases: The worldwide emergence of clone ST131 O25:H4. Int. J. Antimicrob. Agents 2010, 35, 316-321.

47. Rogers, B.A.; Sidjabat, H.E.; Paterson, D.L Escherichia coli O25b-ST131: A pandemic multiresistant community-associated strain. J. Antimicrob. Agents 2011, 66, 1-14.

48. Clark, G.; Paszkiewicz, K.; Hale, J.; Weston, V.; Constantinidou, C.; Penn, C.; Achtman, M.; McNally, A. Genomic analysis uncovers a phenotypically diverse but genetically homogeneous Escherichia coli ST131 clone circulating in unrelated urinary tract infections. J. Antimicrob. Chemother. 2012, 67, 868-877.

49. Hawser, S.P.; Bouchillon, S.K.; Hoban, D.J.; Badal, R.E.; Hsueh, P.R.; Paterson, D.L. Emergence of high-levels of extended-spectrum- $\beta$-lactamase-producing gram-negative bacilli in the Asia-Pacific region: Data from the study for monitoring antimicrobial resistance trends (SMART) program 2007. Antimicrob. Agents Chemother. 2009, 53, 3280-3284. 


\title{
Chapter 4
}

\section{Uncomplicated Urinary Tract Infections in Women in a Sao Paulo Quaternary Care Hospital: Bacterial Spectrum and Susceptibility Patterns}

\section{Marcelo Hisano, Homero Bruschini, Antonio Carlos Nicodemo and Miguel Srougi}

\begin{abstract}
Uncomplicated urinary tract infections (UTI) in women are very common. Regular analysis of bacterial flora is important to formulate updated guidelines. The objective of this study is to determine and compare the microbiology of UTIs and their susceptibility patterns in a quaternary care hospital. In a seven-year review, the urine culture results of 480 female patients with uncomplicated UTIs were analyzed. Patients were divided into three groups according to their diagnosis and treatment characteristics: Group 1, cystitis at outpatient basis; group 2, cystitis at the Emergency Unit; and group 3, pyelonephritis. Group 1 included older patients, with a higher incidence of concomitant diabetes mellitus and recurrent UTIs. E. coli was the most common pathogen, responsible for $75.1 \%$ of cases, mainly for pyelonephritis $(87.3 \%)$. Of the oral antimicrobials tested for cystitis, amoxicillin/clavulanate and nitrofurantoin had the highest susceptibility profiles ( $84.4 \%$ and $87.3 \%$, respectively). For E. coli only, their susceptibility profiles were as high as $90.8 \%$ and $97.4 \%$, respectively. For pyelonephritis treatment, fluoroquinoles had a susceptibility profile $<90 \%$, while ceftriaxone and gentamicin had susceptibility $>90 \%$. Uncomplicated UTI treatment is becoming more challenging because the susceptibility profiles of oral antimicrobials are increasingly resistant. In our environment, cystitis can still be managed with nitrofurantoin. Uncomplicated pyelonephritis should be managed with ceftriaxone or gentamicin.
\end{abstract}

Reprinted from Antibiotics. Cite as: Hisano, M.; Bruschini, H.; Nicodemo, A.C.; Srougi, M. Uncomplicated Urinary Tract Infections in Women in a Sao Paulo Quaternary Care Hospital: Bacterial Spectrum and Susceptibility Patterns. Antibiotics 2014, 3, 98-108.

\section{Introduction}

Urinary tract infections (UTI) in women are very common. The annual incidence was $10.8 \%$ in 2000 , and $60 \%$ of women will have at least one episode during their lifetime [1]. Additionally, after a first episode of urinary tract infection, $44 \%$ of the women will experience another episode within the following year [2].

UTI can be classified as uncomplicated or complicated. Uncomplicated UTI is defined as a UTI that occurs in a woman who has no structural or functional abnormalities, is not pregnant, and has not been instrumented [3]. Anatomically, uncomplicated UTI can be located in the lower urinary tract (cystitis) or in the upper urinary tract (pyelonephritis) [4-6]. 
Usually, uncomplicated cystitis can be managed on an outpatient basis in a primary care health system, while uncomplicated pyelonephritis is usually managed in a hospital setting, followed by outpatient care. At our Institution, a quaternary care hospital located in a developing country, uncomplicated cystitis can be managed either on an office outpatient basis or at the Urological Emergency Unit, according to the needs of the Public Health Care System.

As uncomplicated lower UTIs are usually treated empirically, knowledge of the contemporary flora and pattern of susceptibility is essential and mandatory. While UTI surveillance studies from Europe and the USA are available [4,7-9], equivalent studies from developing countries are sparse [10]. These data are important to formulate guidelines, particularly for such a prevalent disease, as they have the potential to change clinical practice [11]. The aim of this study was to determine the bacterial flora of uncomplicated cystitis and pyelonephritis and compare the susceptibility patterns to identify potential treatment characteristics.

\section{Experimental}

Between January, 2007, and December, 2012, we retrospectively reviewed the clinical records of 160 female patients attended at the Urological Office on an outpatient basis and 2949 female patients treated at the Urological Emergency Unit. Of these patients, we identified and selected for analysis those with a clinical diagnosis of uncomplicated cystitis and pyelonephritis. This review had the approval of the Institutional Ethical Committee.

Uncomplicated cystitis was defined as involving clinical symptoms of dysuria, frequency, urgency, and suprapubic pain, with or without hematuria. Uncomplicated pyelonephritis was defined as clinical symptoms of flank pain, associated with nausea, vomiting, and fever $\left(>37.8^{\circ} \mathrm{C}\right)$, with or without symptoms of cystitis. All patients with these symptoms had a midstream clean catch urine culture. Urine cultures were analyzed, and a colony count of $\geq 10^{3} \mathrm{cfu} / \mathrm{mL}$ was considered positive in cases of cystitis; in the case of pyelonephritis, the threshold was $\geq 10^{4} \mathrm{cfu} / \mathrm{mL}$. Identification of isolates and susceptibility tests were performed on VITEK $^{\circledR} 1$ and 2 automated systems (bioMérieux ${ }^{\circledR}$, Marcy L'Etoile, France). The minimal inhibitory concentrations (MICs) of antimicrobials were determined, and strains were considered susceptible, intermediately susceptible, or resistant according to the breakpoints determined by the Clinical and Laboratory Standards Institute - CLSI [12] at the time of the analysis. The following antimicrobials were tested: amikacin, amoxicillin/clavulanate (A/C), ampicillin, cefepime, cefotaxime, cefoxitin, ceftazidime, ceftriaxone, cephalothin, ciprofloxacin, gentamicin, levofloxacin, nalidixic acid, nitrofurantoin, norfloxacin, piperacillin/tazobactam (Pip/Taz), and sulfamethoxazole/trimethoprim (SMT). For some bacteria, such as Staphylococcus saprophyticus, Streptococcus agalactiae, coagulase-negative Staphylococcus, Corynebacterium spp., and yeast species, susceptibility profiles were not performed because there are no established breakpoints, according to CLSI guidelines [12].

During the review process of the record files, we were able to identify and analyze some of these three clinical characteristics: age ( $<50$ years-old and $\geq 50$ years-old), history of recurrent UTI, and presence of diabetes mellitus (DM). Recurrent UTI was defined as the presence of two or more UTI episodes during a period of six months.

We excluded patients based on the following criteria: being under 14 years-old, having kidney stones larger than $5 \mathrm{~mm}$ or any ureteral or bladder stone, having a UTI less than two months prior 
to the current episode, having recent urinary catheterization, being within the convalescence period of urological surgery; and having a double-J stent, urinary diversion, kidney transplantation, or any congenital urological abnormality without correction. We also excluded patients for whom urine cultures were negative or not available, despite clinical symptoms of uncomplicated UTI.

Three groups were created: group 1 -patients treated at the Urological Office as outpatients with uncomplicated cystitis; group 2-patients treated at the Urological Emergency Unit with uncomplicated cystitis; and group 3-patients treated at the Urological Emergency Unit with uncomplicated pyelonephritis. We then analyzed and compared the microbiological findings of the patients' urine samples.

Statistical analyses of the nonparametric results were performed by a chi-squared test or likelihood ratio test. When the samples were insufficient for chi-squared analysis, the Fisher exact test was performed. A $p$ value of $<0.05$ was considered statistically significant.

\section{Results}

Of the 160 patients attending the Urological Office as outpatients, 103 were included in this study with uncomplicated cystitis. Of the 2949 patients treated at the Urological Emergency Unit, 276 were included in this study with uncomplicated cystitis and 101 with uncomplicated pyelonephritis. Seven patients in group 1, five in group 2, and one in group 3 had two episodes of UTI. The second positive samples from each of those patients were included in the analysis, giving a total of 110 urine samples in group 1, 281 in group 2, and 102 in group 3. The 480 patients included in this study provided 493 urine samples.

The mean ages (and ranges) of patients in groups 1, 2, and 3 were: 55 (18-96) years, 43.2 (15-92) years, and 36 (14-79) years, respectively. The three groups were not equivalent regarding age, history of recurrent UTI or diabetes mellitus. Group 1 had older patients, increased occurrence of recurrent UTI, and greater incidence of diabetes mellitus (Table 1).

Table 1. Demographic data of the three groups and of the total cohort.

\begin{tabular}{|c|c|c|c|c|c|}
\hline \multirow[b]{2}{*}{ Clinical data } & \multicolumn{3}{|c|}{ Groups } & \multirow{2}{*}{$\begin{array}{l}\text { Total } \\
\text { N (\%) }\end{array}$} & \multirow[b]{2}{*}{$p$} \\
\hline & $\begin{array}{c}1 \\
\text { N (\%) }\end{array}$ & $\begin{array}{c}2 \\
\text { N (\%) }\end{array}$ & $\begin{array}{c}3 \\
\text { N (\%) }\end{array}$ & & \\
\hline Patients (N) & 103 & 276 & 101 & 480 & n.a. \\
\hline Age $<50$ years * & $38(36.8)$ & $172(62.3)$ & $81(81.0)$ & $291(60.6)$ & $<0.001$ \\
\hline Recurrent UTI * & $83(76.1)$ & $82(36.0)$ & $22(26.5)$ & $187(44.5)$ & $<0.001$ \\
\hline DM * & $25(22.7)$ & $18(8.5)$ & $2(2.2)$ & $45(10.9)$ & $<0.001$ \\
\hline
\end{tabular}

* Some patients with missing data; n.a.—not applicable; DM-diabetes mellitus.

The microbiology of groups 1, 2, and 3 is summarized in Table 2. In all groups, monomicrobial infection was the most common finding, varying from $92.5 \%$ to $95.1 \%$. Escherichia coli (E. coli) monoinfection was the most common microorganism in monoinfection UTI, varying from $71.2 \%$ to $87.6 \%$. Urinary infection caused by two agents (mixed infection) was identified in $7.3 \%$ of samples in group $1,7.5 \%$ in group 2 , and $4.9 \%$ in group 3 . 
Table 2. Mono and mixed infections in the three groups of patients and in the total cohort.

\begin{tabular}{ccccc}
\hline Urine culture & Group 1 & Group 2 & Group 3 & Total \\
\hline Urine Samples (\%) & $110(22.3)$ & $281(57.0)$ & $102(20.7)$ & $493(100.0)$ \\
Monoinfection (\%) & $102(92.7)$ & $260(92.5)$ & $97(95.1)$ & $459(93.1)$ \\
E. coli $(\%$ monoinfection) & $78(76.5)$ & $185(71.2)$ & $85(87.6)$ & $348(75.8)$ \\
Non-E. coli $(\%$ monoinfection) & $24(23.5)$ & $75(28.8)$ & $12(12.4)$ & $111(24.2)$ \\
\hline Mixed Infection (\%) & $8(7.3)$ & $21(7.5)$ & $5(4.9)$ & $34(6.9)$ \\
$E$. coli $*(\%)$ & $5(62.5)$ & $13(61.9)$ & $4(80.0)$ & $22(64.7)$ \\
Non-E. coli $(\%$ mixed infection) & $3(37.5)$ & $8(38.1)$ & $1(20.0)$ & $12(35.3)$ \\
\hline & $*$ E. coli as one agent.
\end{tabular}

When we analyzed all mono and mixed infection bacterial species together (Table 3), E. coli was still the most frequent pathogen, mainly for group 3 when compared to the other groups $(p=0.004)$. There was also a significantly higher frequency of Staphylococcus saprophyticus in group $2(p=0.004)$.

Table 3. Bacterial spectrum in the three groups of patients and in the entire cohort.

\begin{tabular}{|c|c|c|c|c|c|}
\hline \multirow{3}{*}{ Microorganism } & \multicolumn{3}{|c|}{ Groups } & \multirow{3}{*}{$\begin{array}{c}\text { Total } \\
\text { N (\%) }\end{array}$} & \multirow{3}{*}{$p$} \\
\hline & 1 & 2 & 3 & & \\
\hline & $\mathbf{N}(\%)$ & $\mathbf{N}(\%)$ & $\mathbf{N}(\%)$ & & \\
\hline Escherichia coli & $83(75.5)$ & $198(70.5)$ & $89(87.3)$ & $370(75.1)$ & 0.004 \\
\hline Klebsiella pneumoniae & $7(6.4)$ & $6(2.1)$ & $6(5.9)$ & $19(3.9)$ & 0.074 \\
\hline Enterococcus faecalis & $11(10.0)$ & $18(6.4)$ & $3(2.9)$ & $32(6.5)$ & 0.113 \\
\hline Proteus mirabilis & $3(2.7)$ & $14(5.0)$ & $3(2.9)$ & $20(4.1)$ & 0.472 \\
\hline Staphylococcus saprophyticus & $3(2.7)$ & $28(10.0)$ & $2(2.0)$ & $33(6.7)$ & 0.004 \\
\hline Streptococcus agalactiae & $2(1.8)$ & $11(3.9)$ & $0(0.0)$ & $13(3.3)$ & 0.367 \\
\hline Other & $8(7.3)$ & $26(9.3)$ & $4(3.9)$ & $38(7.7)$ & 0.220 \\
\hline Total & $117(100)$ & $301(100)$ & $107(100)$ & $493(100)$ & n.a \\
\hline
\end{tabular}

n.a: not applicable.

We compared the susceptibility profile of E. coli to all other bacteria in groups 1, 2, and 3 (Table 4). In group 1 , there was a significant difference in susceptibility for $\mathrm{A} / \mathrm{C}$, cefoxitin, nitrofurantoin and SMT. For A/C, the difference was mostly due to a decrease in the resistance profile of $E$. coli compared to other bacteria. Cefoxitin and nitrofurantoin had higher susceptibility for E. coli than for the other bacteria $(96.1 \% \times 62.5 \%$, respectively, for cefoxitin, $p=0.001 ; 97.4 \% \times 30.8 \%$, respectively, for nitrofurantoin, $p<0.001)$. For SMT, the susceptibility of E. coli was lower than the other bacteria (56.2\% and $92.3 \%$, respectively; $p=0.013$ ). In group 2 , we found a significant difference in the susceptibility profile between $E$. coli and other bacteria for A/C $(90.8 \% \times 78.3 \%$, respectively; $p=0.031)$, cefoxitin $(96.5 \% \times 70.6 \%$, respectively; $p=0.002)$, cephalothin $(66.2 \% \times 73.1 \%$, respectively; $p=0.003)$, nitrofurantoin $(94.5 \% \times 23.8 \%$, respectively; $p<0.001)$ and SMT $(64.1 \% \times 85.2 \%$, respectively; $p=0.030)$. In group 3 , when comparing the susceptibility profile of $E$. coli and all other bacteria, there were significant differences in the susceptibility profiles for cefoxitin $(96.4 \% \times 66.7 \%$, respectively; $p=0.043)$ and nitrofurantoin $(95.2 \% \times 42.9 \%$, respectively; $p<0.001)$. Between groups 1,2 , and 3 , when analyzing $E$. coli, there was a 
susceptibility difference for amikacin $(100 \% \times 100 \% \times 92.9 \%$, respectively; $p<0.001)$; when analyzing other bacteria, there was a susceptibility difference for ciprofloxacin $(95.2 \% \times 82.5 \% \times 60 \%$, respectively; $p=0.049$ ).

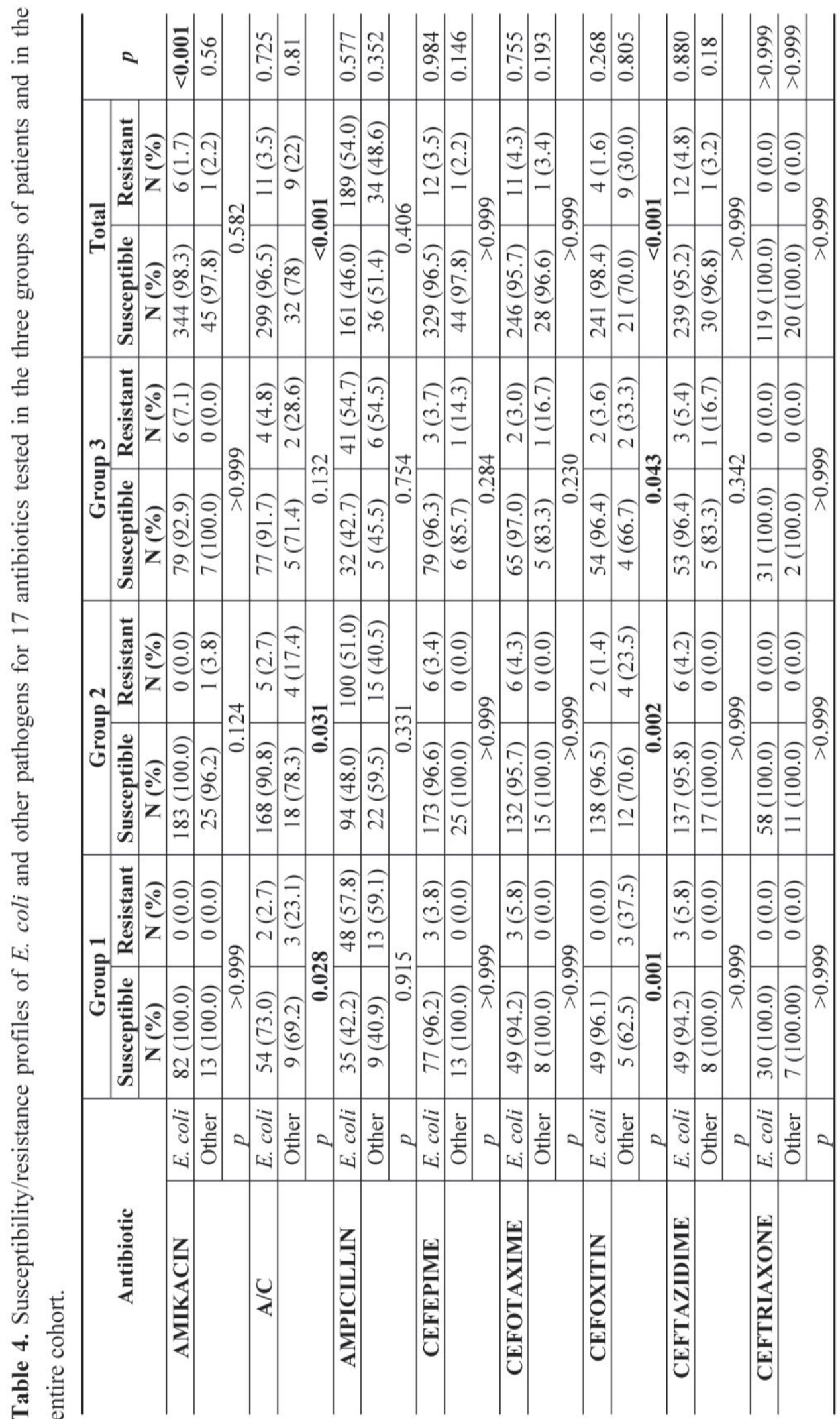




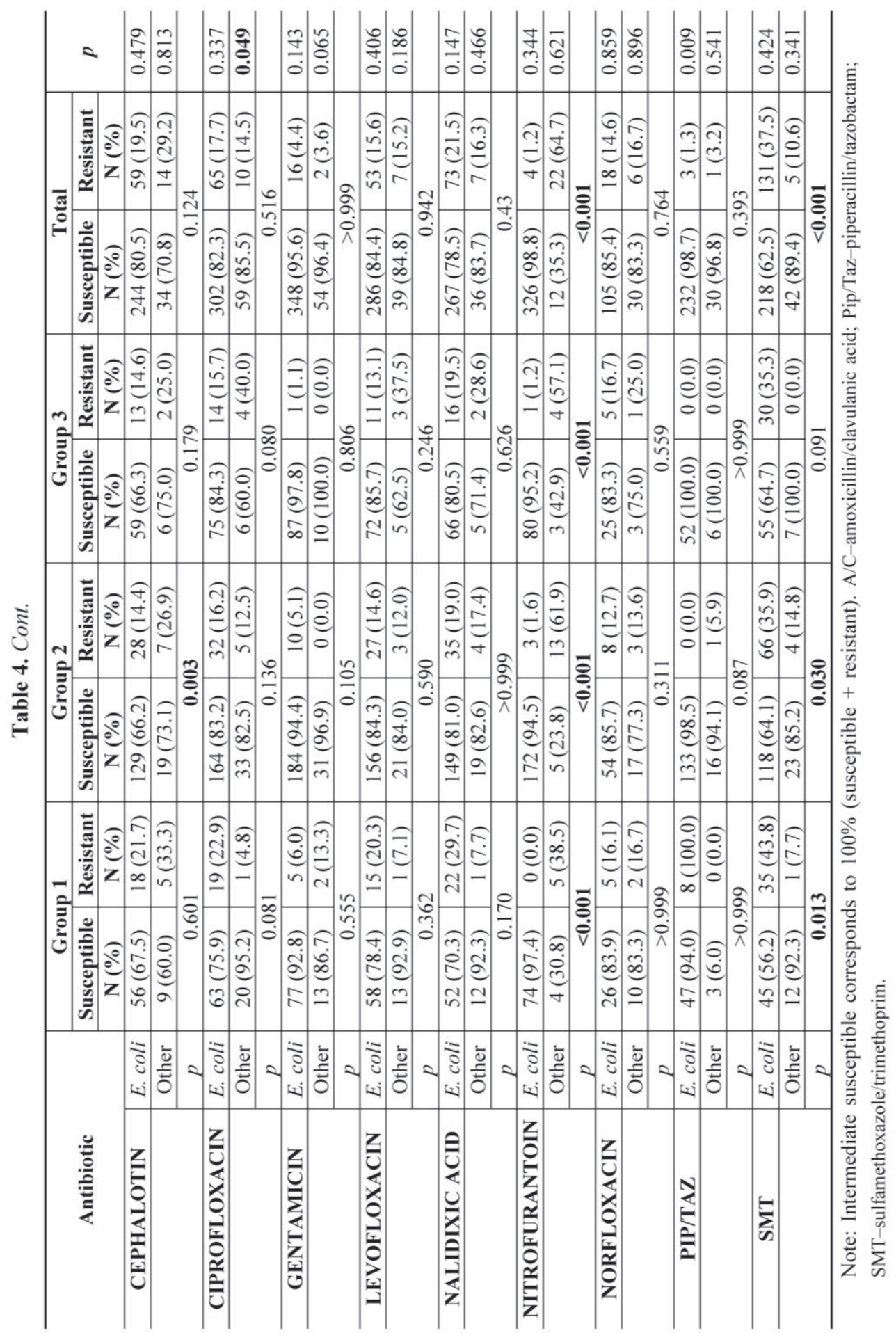

\section{Discussion}

E. coli is the predominant uropathogen in uncomplicated UTI, involved in $75.1 \%$ of cases. For pyelonephritis, $E$. coli is even more common than the other bacteria, with a frequency of $87.3 \%$; some studies have shown that $E$. coli is the responsible pathogen in $80 \%$ to $90 \%$ of these cases $[13,14]$. Although the genetic and behavioral risk factors for cystitis and pyelonephritis are similar, the predominance of $E$. coli causing pyelonephritis can be attributed to its intrinsic pathogenicity and virulence [15]. 
In general, when analyzing the susceptibility profile, there is no oral treatment option with susceptibility higher than $90 \%$, although some authors considered a threshold of $80 \%$ for inclusion in the Clinical Guidelines [4]. A/C and nitrofurantoin were closer to this level, and they are more effective, especially for E. coli. For pyelonephritis treatment, ceftriaxone had a susceptibility of at least $94 \%$; thus, it can be selected to initiate therapy in place of fluoroquinolones.

Our quaternary care hospital has its particularities. The Urological Emergency Unit treats all types of urological emergencies, from acute simple cases to complex urogenital trauma. Because it is not an exclusive referenced emergency, the UTI cases assisted there usually reflect community-acquired infections. The Urological Office takes care of more patients with comorbidities, representing more complex cases. A similar type of UTI classification has been published by Laupland et al. [16], analyzing ambulatory, hospital, and nursing home UTIs. They found E. coli frequencies of $74.2 \%$, $65.5 \%$, and $46.6 \%$ in each of these locations, respectively, as well as a difference in susceptibility profiles between them.

In 2008, a multi-center analysis of the microbiology of uncomplicated cystitis (ARESC) [8] showed an E. coli frequency of $76.7 \%$, close to that found in the present study. The overall susceptibilities to ampicillin, A/C, ciprofloxacin, nalidixic acid, and nitrofurantoin were $45.1 \%$, $82.1 \%, 91.8 \%, 81.4 \%$, and $95.2 \%$, respectively. In the same study, the susceptibility profiles of Brazilian samples for ampicillin, $\mathrm{A} / \mathrm{C}$, ciprofloxacin, nalidixic acid, and nitrofurantoin were $33.8 \%$, $78.7 \%, 89.0 \%, 74.7 \%$, and $84.1 \%$, respectively. In our study considering both groups 1 and 2 together, we found susceptibilities of $47.3 \%, 84.4 \%, 82.1 \%, 78.9 \%$, and $87.3 \%$, respectively, for the same antimicrobials. Our results showed susceptibility profiles for these antimicrobials similar to those found for Brazilian samples, although the period and place of analysis were different (the ARESC study was conducted between September, 2003 and June, 2006 and involved four Brazilians centres).

When we compared E. coli to the other bacteria (mainly Klebsiella pneumonia, Enterococcus faecalis and Proteus mirabilis) in all groups, the susceptibility profiles differed, mainly for $\mathrm{A} / \mathrm{C}$, cefoxitin, and nitrofurantoin. These antimicrobials had higher susceptibility results when the UTI agent was E. coli. In another study of the ARESC group [17], the susceptibility profiles of Brazilian E. coli samples for ampicillin, A/C, ciprofloxacin, nalidixic acid, nitrofurantoin, and SMT were $37.7 \%, 79.8 \%, 89.2 \%, 75.4 \%, 94.3 \%$, and $54.5 \%$, respectively. For the same antimicrobials, our study found susceptibilities of $46.0 \%, 96.5 \%, 82.3 \%, 78.5 \%, 98.8 \%$, and $62.5 \%$, respectively. A similar result was found by Linhares et al. [18] for nitrofurantoin and pivmecillinam, with the same pattern of high susceptibility for $E$. coli but low for non-E. coli, although they analyzed male and female patients. Nitrofurantoin is known to have no activity against Proteus spp. and Pseudomonas aeruginosa [19]; this information is in line with our results because $P$. mirabilis was analyzed as a non-E. coli agent. Interestingly, although SMT had a low susceptibility profile for $E$. coli $(56.2 \%, 64.1 \%$, and $64.7 \%$ for groups 1,2 , and 3, respectively), it had a higher susceptibility profile to non- $E$. coli agents, reaching $92.3 \%, 85.2 \%$, and $100 \%$ susceptibility in groups 1,2 , and 3 , respectively ( $p<0.05$ for groups 1 and 2 only). These findings may indicate that SMT cannot be considered for E. coli treatment but can be used for non-E. coli bacteria, although others studies did not find similar results $[17,18]$.

Our study has some limitations. The retrospective nature of this analysis has an intrinsic bias. As we retrospectively analyzed six years of uncomplicated UTI, some changes in MIC occurred 
during these years according to CLSI. We are aware of this fact, but as we considered final outcomes of the test (Susceptible, Intermediately Susceptible, and Resistant) to treat the patients at that moment successfully, we considered it clinically relevant; other groups had already utilized this classification for the results of susceptibility tests $[10,16,18]$. Another limitation is the fact that susceptibility profiles were not performed for some bacteria.

The three groups were not statistically similar; group 1 had older patients with higher incidences of diabetes mellitus and recurrent UTI than groups 2 and 3. This difference adds another source of bias to this study, however, as already mentioned, this difference was due to our local health care organization. Additionally, confirming our perception, this group division resembled a comparison between uncomplicated UTIs from a quaternary care hospital (group 1) and community-acquired uncomplicated UTIs (groups 2 and 3).

The 2013 European Guideline on urological infections [5] outlines the antimicrobials of choice for the treatment of uncomplicated cystitis: fosfomycin trometamol, pivmecillinam and nitrofurantoin. Our study did not test fosfomycin trometamol or pivmecillinam; the latter is not available in our country. Fosfomycin trometamol is not routinely tested at our hospital because, at the time of urine culture analysis, there were no established MIC breakpoints for some bacteria and antimicrobials according to CLSI guidelines [12]. Additionally, due to the retrospective nature of this study, we were unable to perform retests. Nitrofurantoin had a general susceptibility profile close to $90 \%$, but for $E$. coli, it was at least $94.5 \%$. In our environment, $\mathrm{A} / \mathrm{C}$ and ciprofloxacin had susceptibilities of $84.4 \%$ and $82.1 \%$, respectively; both were at the lower limit for inclusion as first options for cystitis treatment. Although some European countries reported similar susceptibility profiles, these antibiotics are recommended by their national guidelines [20].

For uncomplicated pyelonephritis, the Infectious Disease Society of America Guidelines [4] and the 2013 European Guideline [5] recommend fluoroquinolones as alternative therapeutic agents. Our study showed a susceptibility profile lower than $90 \%$ for these antimicrobials, making them unsuitable for our environment because the susceptibility threshold to be considered as a first line therapy should be higher than $90 \%[4,6]$. The alternatives for empirical antimicrobial therapy are ceftriaxone, with a susceptibility of $100 \%$, or gentamicin, with a susceptibility of $98.0 \%$. Although nitrofurantoin had an acceptable susceptibility profile for pyelonephritis treatment, mainly for E. coli, due to its rapid renal excretion and insufficient therapeutic blood level, it is not indicated to treat pyelonephritis [21].

\section{Conclusions}

Uncomplicated UTI treatment is becoming more difficult to manage because the susceptibility profiles of current oral antimicrobials are becoming more resistant. For simple cystitis, nitrofurantoin is at the lower limit of acceptable susceptibility to continue to be an antimicrobial of choice, but it is still very active for E. coli, as well as A/C. For pyelonephritis, fluoroquinolones are not suitable as a first line therapy and should be replaced by ceftriaxone or gentamicin. Judicious use of antimicrobials, adhesion to guidelines, and new drug alternatives should be considered as strategies to avoid increasing resistance. 


\section{Acknowledgments}

The authors thank Elisa Cruz for her helpful assistance and Rogério Ruscitto for his statistical analysis assistance.

\section{Author Contributions}

Marcelo Hisano worked on collecting data and preparing the manuscript. Homero Bruschini worked on collecting data, preparing and reviewing the manuscript. Antonio Carlos Nicodemo worked on preparing and reviewing the manuscript. Miguel Srougi worked on preparing and reviewing the manuscript.

\section{Conflicts of Interest}

The authors declare no conflicts of interest.

\section{References}

1. Foxman, B.; Barlow, R.; D’Arcy, H.; Gillespie, B.; Sobel, J.D. Urinary tract infection: Self-reported incidence and associated costs. Ann. Epidemiol. 2000, 10, 509-515.

2. Ikäheimo, R.; Siitonen, A.; Heiskanen, T.; Kärkkäinen, U.; Kuosmanen, P.; Lipponen, P.; Mäkelä, P.H. Recurrence of urinary tract infection in a primary care setting: Analysis of a 1-year follow-up of 179 women. Clin. Infect. Dis. 1996, 22, 91-99.

3. Foxman, B. The epidemiology of urinary tract infection. Nat. Rev. Urol. 2010, 7, 653-660.

4. Gupta, K.; Hooton, T.M.; Naber, K.G.; Wullt, B.; Colgan, R.; Miller, L.G.; Moran, G.J.; Nicolle, L.E.; Raz, R.; Schaeffer, A.J.; et al. International clinical practice guidelines for the treatment of acute uncomplicated cystitis and pyelonephritis in women: A 2010 update by the Infectious Diseases Society of America and the European Society for Microbiology and Infectious Diseases. Clin. Infect. Dis. 2011, 52, e103-e120.

5. Grabe, M.; Bjerklund-Johansen, T.E.; Botto, H.; Çek, M.; Naber, K.G.; Pickard, R.S.; Tenke, P.; Wagenlehner, F.; Wullt, B. EAU Guidelines on Urological Infection. 2013. Available online: http://www.uroweb.org/gls/pdf/18_Urological\%20infections_LR.pdf (accessed on 30 December 2013).

6. Colgan, R.; Williams, M.; Johnson, J.R. Diagnosis and treatment of acute pyelonephritis in women. Am. Fam. Physician 2011, 84, 519-526.

7. Kahlmeter, G. An international survey of the antimicrobial susceptibility of pathogens from uncomplicated urinary tract infections: the ECO-SENS Project. J. Antimicrob. Chemother. 2003, 51, 69-76.

8. Naber, K.G.; Schito, G.; Botto, H.; Palou, J.; Mazzei, T. Surveillance study in Europe and Brazil on clinical aspects and Antimicrobial Resistance Epidemiology in Females with Cystitis (ARESC): Implications for empiric therapy. Eur. Urol. 2008, 54, 1164-1175.

9. Kahlmeter, G.; Poulsen, H.O. Antimicrobial susceptibility of Escherichia coli from community-acquired urinary tract infections in Europe: The ECO-SENS study revisited. Int. J. Antimicrob. Agents 2012, 39, 45-51. 
10. Kiffer, C.R.; Mendes, C.; Oplustil, C.P.; Sampaio, J.L. Antibiotic resistance and trend of urinary pathogens in general outpatients from a major urban city. Int. Braz. J. Urol. 2007, 33, $42-48$.

11. Masterton, R. The importance and future of antimicrobial surveillance studies. Clin. Infect. Dis. 2008, 47, S21-S31.

12. Clinical and Laboratory Standards Institute. performance standards for antimicrobial susceptibility testing; seventeenth to twenty-second informational supplement. CLSI document M100-S17-22. Clinical and Laboratory Standards Institute: Wayne, PA, USA, 2007-2012.

13. Czaja, C.A.; Scholes, D.; Hooton, T.M.; Stamm, W.E. Population-based epidemiologic analysis of acute pyelonephritis. Clin. Infect. Dis. 2007, 45, 273-280.

14. Nicolle, L.E. Uncomplicated urinary tract infection in adults including uncomplicated pyelonephritis. Urol. Clin. North Am. 2008, 35, 1-12.

15. Wiles, T.J.; Kulesus, R.R.; Mulvey, M.A. Origins and virulence mechanisms of uropathogenic Escherichia coli. Exp. Mol. Pathol. 2008, 85, 11-19.

16. Laupland, K.B.; Ross, T.; Pitout, J.D.; Church, D.L.; Gregson, D.B. Community-onset urinary tract infections: A population-based assessment. Infection 2007, 35, 150-153.

17. Schito, G.C.; Naber, K.G.; Botto, H.; Palou, J.; Mazzei, T.; Gualco, L.; Marchese, A. The ARESC study: An international survey on the antimicrobial resistance of pathogens involved in uncomplicated urinary tract infections. Int. J. Antimicrob. Agents 2009, 34, 407-413.

18. Linhares, I.; Raposo, T.; Rodrigues, A.; Almeida, A. Frequency and antimicrobial resistance patterns of bacteria implicated in community urinary tract infections: A ten-year surveillance study (2000-2009). BMC Infect. Dis. 2013, 13, e19.

19. Wagenlehner, F.M.; Weidner, W.; Naber, K.G. Antibiotics in urology: New essentials. Urol. Clin. North Am. 2008, 35, 69-79.

20. Haslund, J.M.; Dinesen, M.R.; Nielsen, A.B.S.; Llor, C.; Bjerrum, L. Different recommendations for empiric first-choice antibiotic treatment of uncomplicated urinary tract infections in Europe. Scand. J. Prim. Health Care 2013, 31, 235-240.

21. Shepherd, A.K.; Pottinger, P.S. Management of urinary tract infections in the era of increasing antimicrobial resistance. Med. Clin. North Am. 2013, 97, 737-757. 


\title{
Chapter 5
}

\section{Adult and Pediatric Intra-Institutional Trends of Ciprofloxacin Susceptibility in $E$. coli Positive Urinary Cultures}

\author{
Winifred Owumi, Niaz Banaei and Linda D. Shortliffe
}

\begin{abstract}
Antimicrobial drug resistance in treatment of urinary tract infection (UTI) continues to rise worldwide. To examine contributions of physician prescribing patterns to fluoroquinolone (ciprofloxacin, CP) resistance, we examined Escherichia coli (E. coli) resistance patterns in urinary cultures. Since CP usage is limited in children, we compared CP resistance trends in adults and children to those of more commonly used trimethoprim-sulfamethoxazole (TMP-SMX) and nitrofurantoin (NF). Our data show that although the general pediatric population has lower resistance to ciprofloxacin, resistance levels are rising with increased usage. While NF susceptibility is historically stable, TMP-SMX resistance is slightly higher in children compared to adults. In both adults and children, antimicrobial resistance patterns vary according to clinical practice site, with ambulatory urology patients showing the highest resistance. This suggests that physician's prescribing patterns contribute to antimicrobial resistance.
\end{abstract}

Reprinted from Antibiotics. Cite as: Owumi, W.; Banaei, N.; Shortliffe, L.D. Adult and Pediatric Intra-Institutional Trends of Ciprofloxacin Susceptibility in E. coli Positive Urinary Cultures. Antibiotics 2014, 3, 163-173.

\section{Introduction}

Antimicrobial drug resistance is a major concern in medicine. Urinary tract infections (UTIs) are common in both adults and children and are usually treated empirically. As a result, antimicrobial susceptibility is important for guiding therapy selection. Since susceptibility trends vary among countries, hospitals, clinical practice sites (ambulatory versus hospital), and patient groups (adult versus child), understanding these trends is important to infection management. While microbial resistance may be innate or acquired from spontaneous or induced mutation, contributions of physician usage patterns on selective pressures are unclear. To better understand this association, we investigated patterns of urinary Escherichia coli (E. coli) antimicrobial susceptibility within the same institution in different medical specialty practice sites, with the assumption that these sites reflect elements of physician specialists' usage patterns. Since ciprofloxacin (CP) usage is limited in children, we compared $\mathrm{CP}$ resistance in adults and children to two other commonly used antimicrobial agents: trimethoprim-sulfamethoxazole (TMP-SMX) and nitrofurantoin (NF). 


\section{Results}

\subsection{Urinary Cultures}

A total of 156,730 urinary cultures were examined. Of these, $38,890(24.8 \%)$ were pediatric cultures (annual average of $3889 \pm 230$ [SD, Standard deviation]) and 117,840 (75.2\%) were adult (annually 11,784 \pm 1752 ). Of these, 7414 cultures $(19.1 \%)$ were positive for $E$. coli from the pediatric hospital and 26,582 from adult cultures (22.6\%). The majority of these were from females: $5979(80.2 \pm 3.8 \%)$ from girls, and 22,950 (86.7 $\pm 1.6 \%)$ from adult females (Table 1). Most urinary specimens positive for $E$. coli were from the ambulatory outpatient clinic and distributed as shown (Table 2).

Table 1. Patient demographics $(\mathrm{N}=$ number$)$.

\begin{tabular}{ccccc}
\hline Group & Pediatric $\mathbf{N}$ & $\mathbf{\%}$ & Adult N & \% \\
\hline Total positive urine culture & 38,890 & 24.8 & 117,840 & 75.2 \\
E. coli positive & 7414 & 19.1 & 26,582 & 22.6 \\
Females & 5979 & 80.2 & 22,950 & 86.7 \\
Males & 1416 & 19.8 & 3433 & 13.3 \\
Unknown gender & 19 & 0.2 & 199 & 0.8 \\
\hline
\end{tabular}

Table 2. E. coli positive urinary cultures by clinical site $(\mathrm{N}=$ number, $\mathrm{OPT}=$ outpatient, $\mathrm{INPT}=$ inpatient, ED = emergency department, Gen Peds = general pediatrics, Gen Med = general medicine, $\mathrm{OB}=$ obstetrics, $\mathrm{GYN}=$ gynecology, $\mathrm{URO}=$ urology).

\begin{tabular}{ccccc}
\hline Group & Pediatric $\mathbf{N}$ & $\mathbf{\%}$ & Adult $\mathbf{N}$ & \% \\
\hline All Treatment Sites & 7414 & 19.1 & 26,582 & 22.6 \\
OPT & 6245 & 84.2 & 22,868 & 86 \\
INPT & 639 & 8.6 & 3709 & 14 \\
ED & 1719 & 23.2 & 5444 & 20.5 \\
Gen Peds/Gen Med & 1267 & 17.1 & 12,616 & 47.5 \\
OB/GYN & 698 & 9.4 & 917 & 3.4 \\
URO & 283 & 3.8 & 1703 & 6.4 \\
\hline
\end{tabular}

\subsection{Distribution of Antibiotics Susceptibilities}

Urinary E. coli was more likely to be susceptible to ciprofloxacin (CP) in children compared to adults $(p<0.002)$. Both child and adult sites showed decreasing susceptibilities from 100\% susceptibility in 2002 to $90 \%$ in 2011 in children, as compared to $74 \%$ in adults (Figure 1). Outpatient (OPT) urine cultures had higher susceptibility to CP than inpatient specimen in both children and adults, with a larger difference in adults (Figure 2). Susceptibility also varied among ambulatory sites, with the lowest susceptibilities in the Urology clinic specimens ( $73 \%$ in children) as compared to other sites that remained between 88 and 95\%. Adult urology ambulatory specimens showed a drop in susceptibility to 55\% by 2011 (Tables 3 and 4 and Figure 3a). Similarly by 2011 inpatient hospital specimens were only $60 \%$ susceptible to ciprofloxacin (Table 4 and Figure $3 \mathrm{~b}$ ). All $p$-values were $<0.02$. 
Figure 1. Adult and pediatric ciprofloxacin susceptibility trends for E. coli positive urinary cultures.

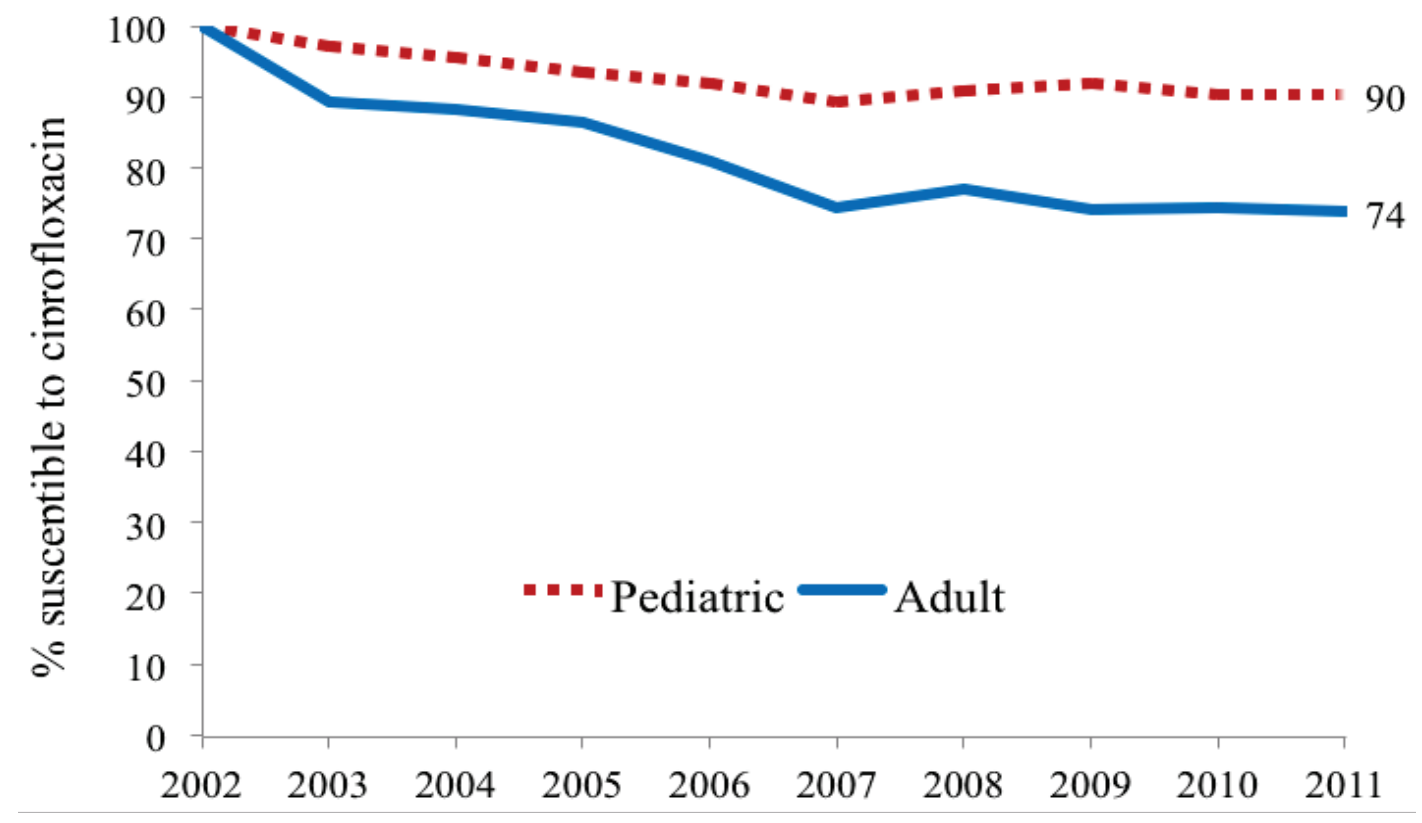

Figure 2. Outpatient and inpatient adult and pediatric ciprofloxacin susceptibility trends for $E$. coli positive urinary cultures $(\mathrm{OPT}=$ outpatient, $\mathrm{INPT}=$ inpatient $)$.

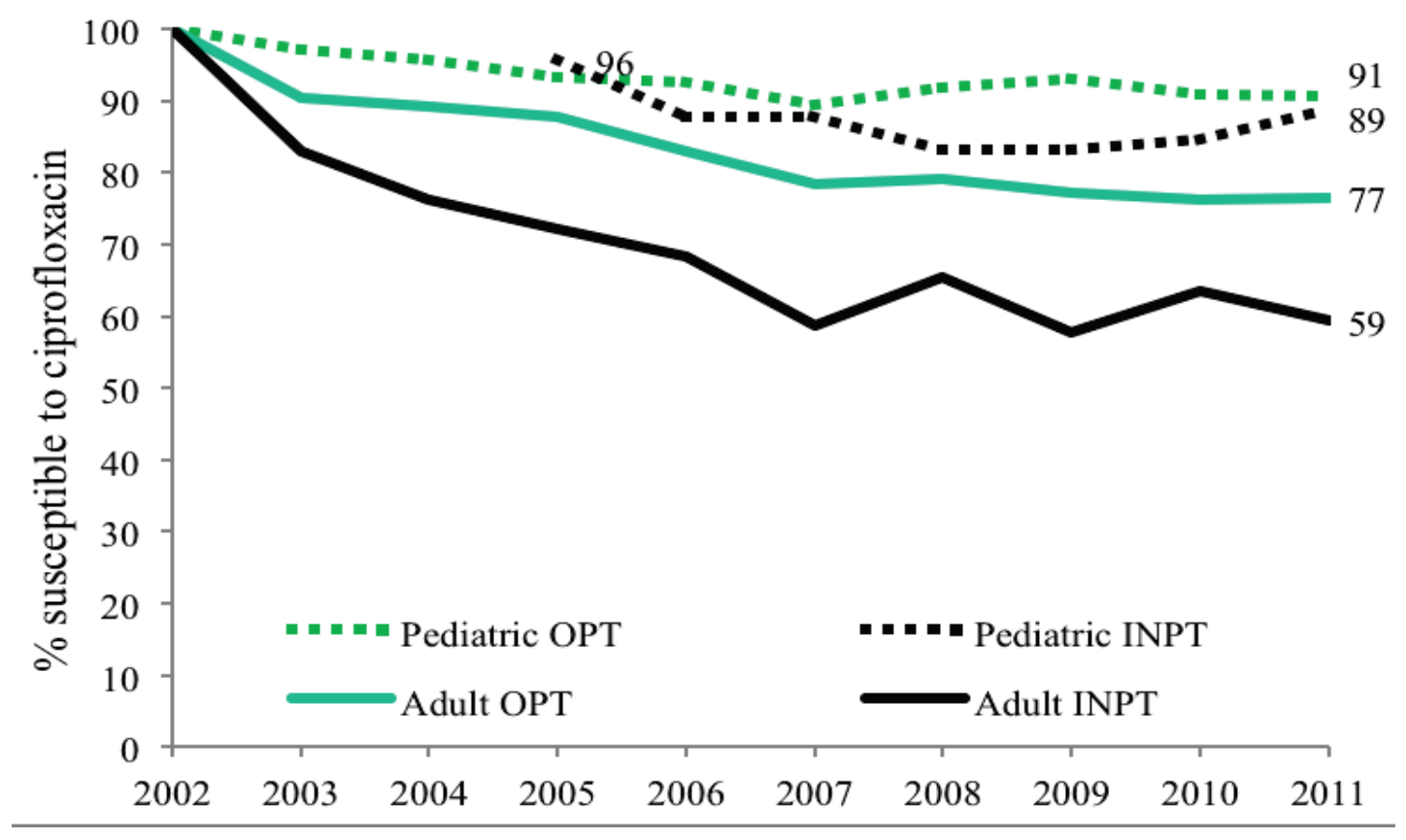

Trimethoprim-sulfamethoxazole (TMP-SMX) susceptibility is lower in children than adults. While TMP-SMX susceptibility declined during the study period, the rate of decrease was lower than for CP. Again, susceptibility varied with clinical treatment site and lowest susceptibility occurred in pediatric and adult urology ambulatory sites (Figure 4a,b, $p<0.02$ ). E. coli NF susceptibility for both children and adult specimens was, meanwhile, relatively stable varying from $93 \%-100 \%$ between 2002 and 2011 without significant difference between adults and children nor among treatment sites. Comparison of these three commonly used antibiotics within ambulatory urology 
demonstrated three patterns of $E$. coli susceptibility: (a) no differences in NF susceptibility between children and adults; (b) lower TMP-SMX susceptibilities in children than adults; and (c) higher CP susceptibility in children than adults Figures 2-5).

Table 3. Ciprofloxacin susceptibilities of pediatric E. coli positive urinary cultures by site $(\mathrm{N}=$ number, OPT $=$ outpatient, INPT = inpatient, $\mathrm{ED}=$ emergency department, Gen Peds $=$ general pediatrics, $\mathrm{OB}=$ obstetrics, $\mathrm{URO}=$ urology).

\begin{tabular}{|c|c|c|c|c|c|c|c|c|}
\hline Year & $\begin{array}{c}\text { All E. coli } \\
\text { Cultures N }\end{array}$ & $\begin{array}{c}\text { All Sites } \\
\mathbf{N}(\%)\end{array}$ & $\begin{array}{c}\text { OPT } \\
\text { N (\%) } \\
\end{array}$ & $\begin{array}{l}\text { INPT } \\
\text { N (\%) } \\
\end{array}$ & $\begin{array}{c}\text { ED } \\
\text { N (\%) } \\
\end{array}$ & $\begin{array}{c}\text { Gen Peds } \\
\text { N (\%) } \\
\end{array}$ & $\begin{array}{c}\text { OB N } \\
(\%) \\
\end{array}$ & $\begin{array}{c}\text { URO N } \\
(\%) \\
\end{array}$ \\
\hline 2002 & 528 & $490(100)$ & N/A & N/A & $93(100)$ & N/A & N/A & $\mathrm{N} / \mathrm{A}$ \\
\hline 2003 & 577 & $519(97)$ & N/A & N/A & $109(100)$ & N/A & N/A & N/A \\
\hline 2004 & 1074 & $861(95.5)$ & N/A & N/A & $113(99.1)$ & N/A & N/A & N/A \\
\hline 2005 & 1144 & $968(93.5)$ & $876(93.4)$ & $90(95.7)$ & $114(95)$ & $499(95.6)$ & $94(94)$ & $20(69)$ \\
\hline 2006 & 680 & $584(92)$ & $526(92.4)$ & $58(87.9)$ & $132(95.7)$ & $146(94.2)$ & 94 (94.9) & $29(85.3)$ \\
\hline 2007 & 632 & $520(89.2)$ & $448(89.4)$ & $72(87.8)$ & $141(97.2)$ & $112(94.9)$ & $88(89.8)$ & $32(84.2)$ \\
\hline 2008 & 631 & $518(90.9)$ & $464(91.9)$ & $54(83.1)$ & $162(93.1)$ & $103(95.4)$ & $93(95.9)$ & $18(81.8)$ \\
\hline 2009 & 749 & $623(92)$ & $563(93.1)$ & $60(83.3)$ & $255(96.2)$ & 85 (95.5) & $78(95.1)$ & $29(87.9)$ \\
\hline 2010 & 731 & $571(90.2)$ & $510(90.9)$ & $61(84.7)$ & $224(98.2)$ & $74(100)$ & $93(96.9)$ & $29(76.3)$ \\
\hline 2011 & 668 & $554(90.4)$ & $468(90.7)$ & $87(88.8)$ & $189(95.5)$ & $94(93.1)$ & $80(95.2)$ & $27(73)$ \\
\hline Total & 7414 & 6208 & 5718 & 489 & 1532 & 1482 & 620 & 198 \\
\hline Mean & 741 & $621(93)$ & $572(93)$ & $61(87)$ & $153(97)$ & $185(96)$ & $89(95)$ & $20(80)$ \\
\hline SD & 205 & $162(3)$ & $158(3)$ & $26(4)$ & $54(2)$ & $159(2)$ & $7(2)$ & $11(7)$ \\
\hline
\end{tabular}

Table 4. Ciprofloxacin susceptibilities of adult E. coli positive urinary cultures by site $(\mathrm{N}=$ number, OPT $=$ outpatient, INPT $=$ inpatient, ED = emergency department, Gen $\mathrm{Med}=$ general medicine, GYN = gynecology, $\mathrm{URO}=$ urology).

\begin{tabular}{ccccccccc}
\hline Year & $\begin{array}{c}\text { All } \boldsymbol{E} \text { c coli } \\
\text { Cultures } \mathbf{N}\end{array}$ & $\begin{array}{c}\text { All Sites } \\
\mathbf{N}(\%)\end{array}$ & $\begin{array}{c}\text { OPT } \\
\mathbf{N}(\boldsymbol{\%})\end{array}$ & $\begin{array}{c}\text { INPT } \\
\mathbf{N}(\mathbf{\%})\end{array}$ & $\begin{array}{c}\text { EDN } \\
\mathbf{( \% )}\end{array}$ & $\begin{array}{c}\text { Gen Med } \\
\mathbf{N}(\%)\end{array}$ & $\begin{array}{c}\text { GYN } \\
\mathbf{N}(\%)\end{array}$ & $\begin{array}{c}\text { URO } \\
\mathbf{N}(\boldsymbol{\%})\end{array}$ \\
\hline 2002 & 1557 & $1306(100)$ & $1083(100)$ & $223(100)$ & $268(100)$ & $470(100)$ & $70(100)$ & $123(100)$ \\
\hline 2003 & 1538 & $1233(89.2)$ & $1061(90.3)$ & $168(82.8)$ & $333(88.8)$ & $501(96.7)$ & $44(89.8)$ & $101(77.7)$ \\
\hline 2004 & 3484 & $2683(88.2)$ & $2500(89.2)$ & $183(76.3)$ & $344(88.4)$ & $1953(91.8)$ & $30(83.3)$ & $104(68.9)$ \\
\hline 2005 & 4456 & $3518(86.4)$ & $3270(87.7)$ & $248(72.1)$ & $338(81.3)$ & $2700(90.4)$ & $50(87.7)$ & $97(68.3)$ \\
\hline 2006 & 2425 & $1817(81)$ & $1599(83.1)$ & $218(68.3)$ & $376(78.7)$ & $949(89.6)$ & $48(85.7)$ & $96(64.9)$ \\
\hline 2007 & 2298 & $1589(74.5)$ & $1345(78.3)$ & $244(58.7)$ & $367(77.1)$ & $722(87.8)$ & $62(80.5)$ & $98(58.7)$ \\
\hline 2008 & 2540 & $1816(76.9)$ & $1571(79.1)$ & $245(65.5)$ & $454(77.5)$ & $825(88)$ & $67(73.6)$ & $89(55.3)$ \\
\hline 2009 & 2702 & $1852(74.1)$ & $1631(77.1)$ & $221(57.7)$ & $470(68.5)$ & $841(90.3)$ & $79(77.5)$ & $96(61.5)$ \\
\hline 2010 & 2425 & $1847(74.3)$ & $1621(76.1)$ & $226(63.5)$ & $466(74.1)$ & $789(85.2)$ & $116(88.5)$ & $71(47)$ \\
\hline 2011 & 2857 & $1955(74)$ & $1712(76.6)$ & $239(59.5)$ & $499(72.1)$ & $770(85.9)$ & $141(87.6$ & $92(55.4)$ \\
\hline Total & 26582 & 19616 & 17396 & 2216 & 3715 & 10520 & 707 & 967 \\
\hline Mean & 2658 & $1962(82)$ & $1740(84)$ & $222(70)$ & $392(81)$ & $1052(91)$ & $71(85)$ & $97(66)$ \\
\hline SD & 858 & $676(9)$ & $670(8)$ & $27(13)$ & $76(9)$ & $710(5)$ & $34(7)$ & $13(15)$ \\
\hline
\end{tabular}


Figure 3. (a) Outpatient pediatric ciprofloxacin susceptibility trends for E. coli positive urinary cultures $(\mathrm{ED}=$ emergency department, All Gen Peds clinics $=$ general pediatrics clinics, $\mathrm{OB}=$ Obstetrics clinics/Labor and delivery, URO = urology clinics); (b) Outpatient adult ciprofloxacin susceptibility trends for E. coli positive urinary cultures (Students' health = undergraduate and graduate students' clinic, ED = emergency department, All med clinics = general medicine clinics, GYN = gynecology clinics, $\mathrm{URO}=$ urology clinics).

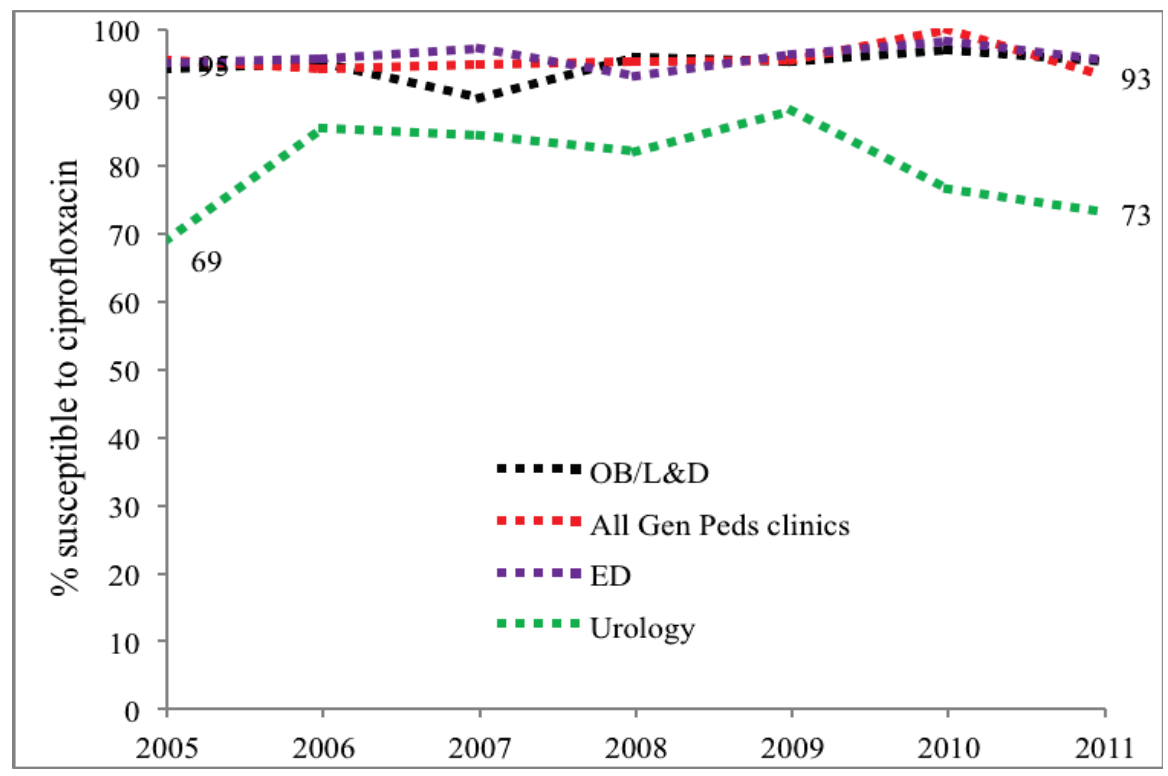

(a)

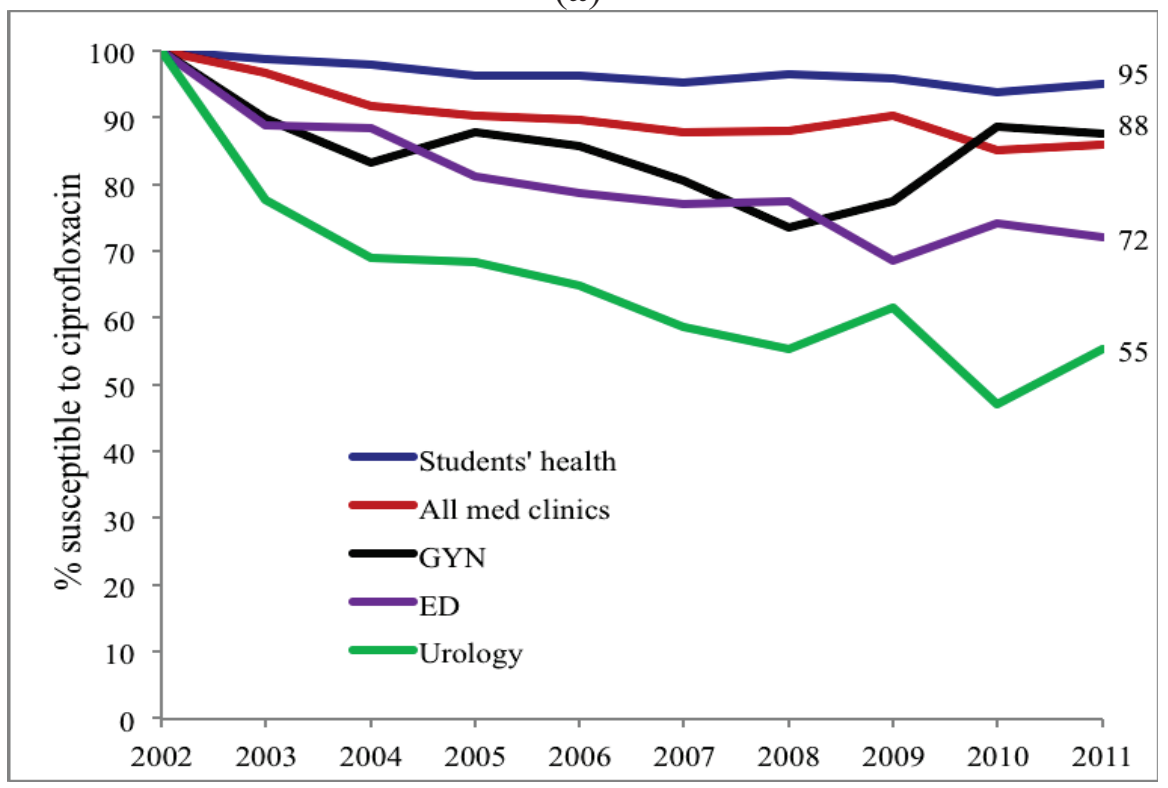

(b) 
Figure 4. (a) Outpatient pediatric TMP-SMX susceptibility trends for E. coli positive urinary cultures $(E D=$ emergency department, All Gen Peds clinics $=$ general pediatrics clinics, $\mathrm{OB}=$ obstetrics clinics/Labor and delivery, $\mathrm{URO}=$ urology clinics); (b) Outpatient adult TMP-SMX susceptibility trends for E. coli positive urinary cultures (Students' health = undergraduate and graduate students' clinic, ED = emergency department, All med clinics = general medicine clinics, GYN = gynecology clinics, $\mathrm{URO}=$ urology clinics).

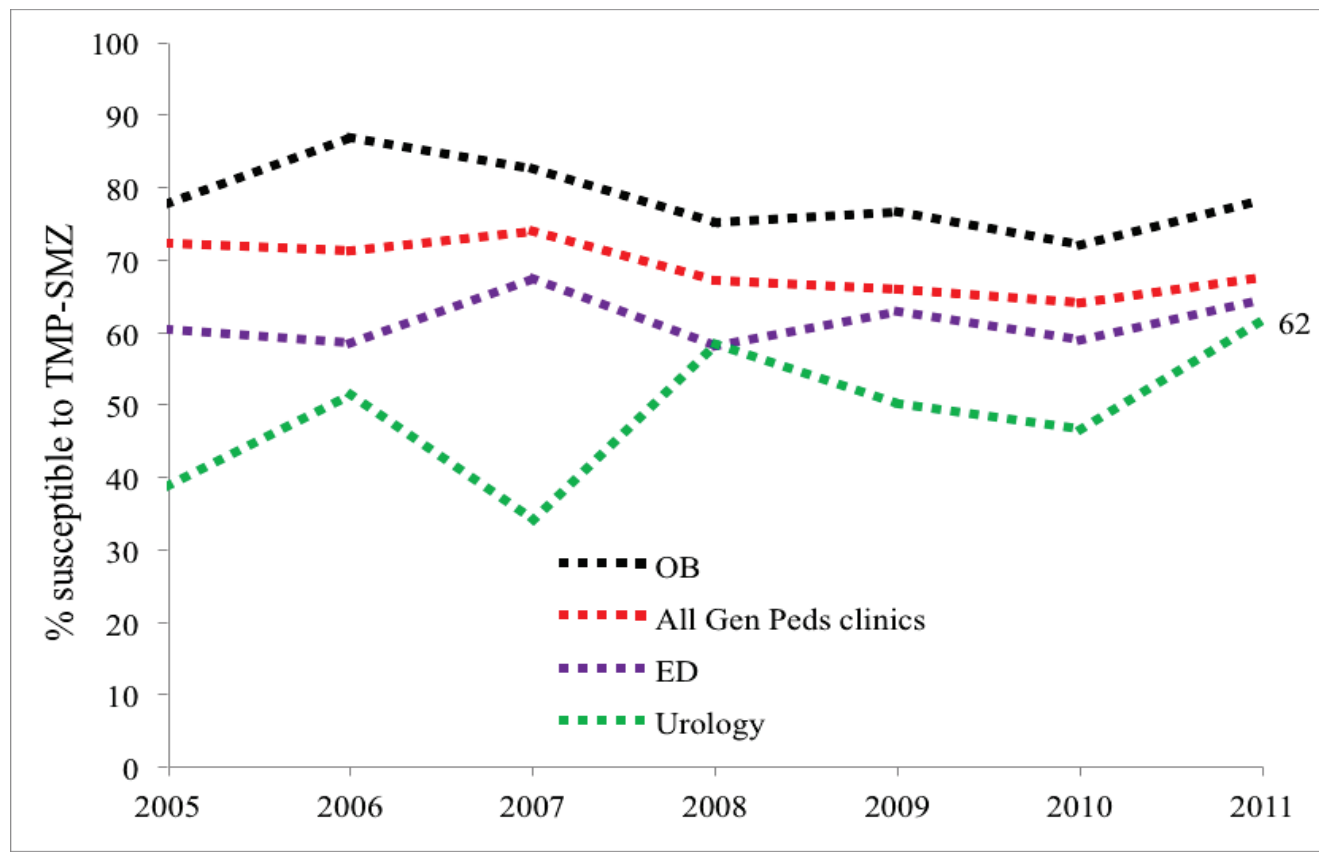

(a)

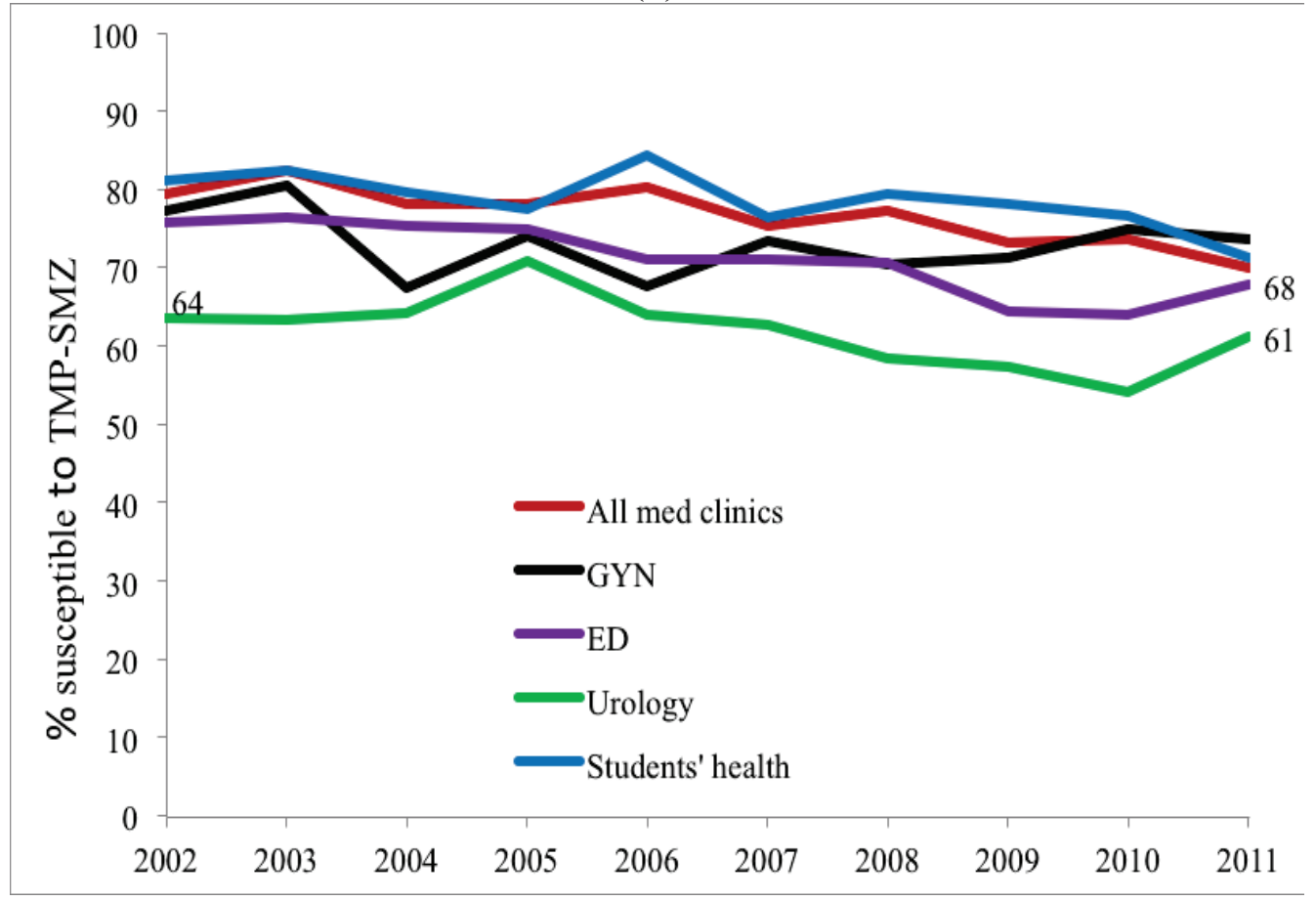

(b) 
Figure 5. (a) Pediatric urology clinic susceptibility trends for three commonly prescribed antimicrobial agents for $E$. coli positive urinary cultures $(\mathrm{NF}=$ nitrofurantoin, $\mathrm{CP}=$ ciprofloxacin, TMP-SMX = trimethoprim-sulfamethoxazole); (b) Adult urology clinic susceptibility trends for three commonly prescribed antimicrobial agents for E. coli positive urinary cultures $(\mathrm{NF}=$ nitrofurantoin, $\mathrm{CP}=$ ciprofloxacin, TMP-SMX $=$ trimethoprim-sulfamethoxazole).

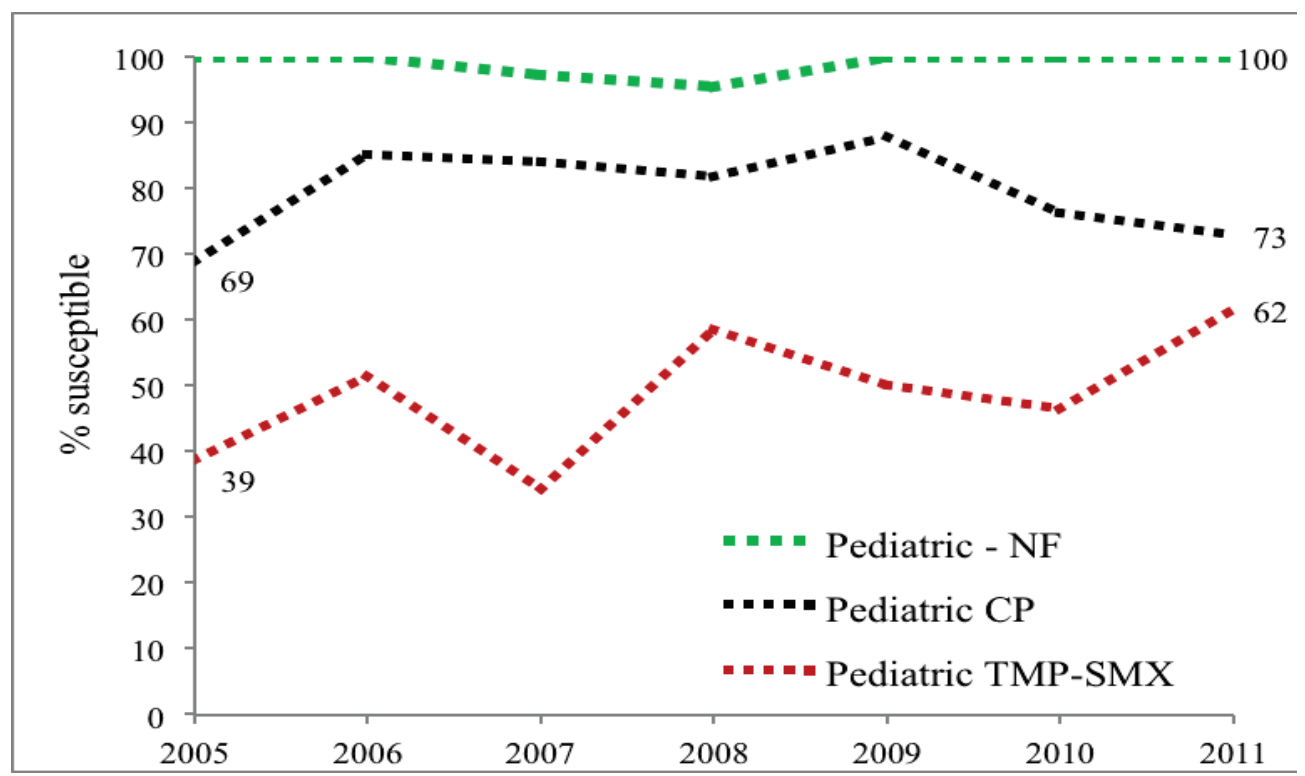

(a)

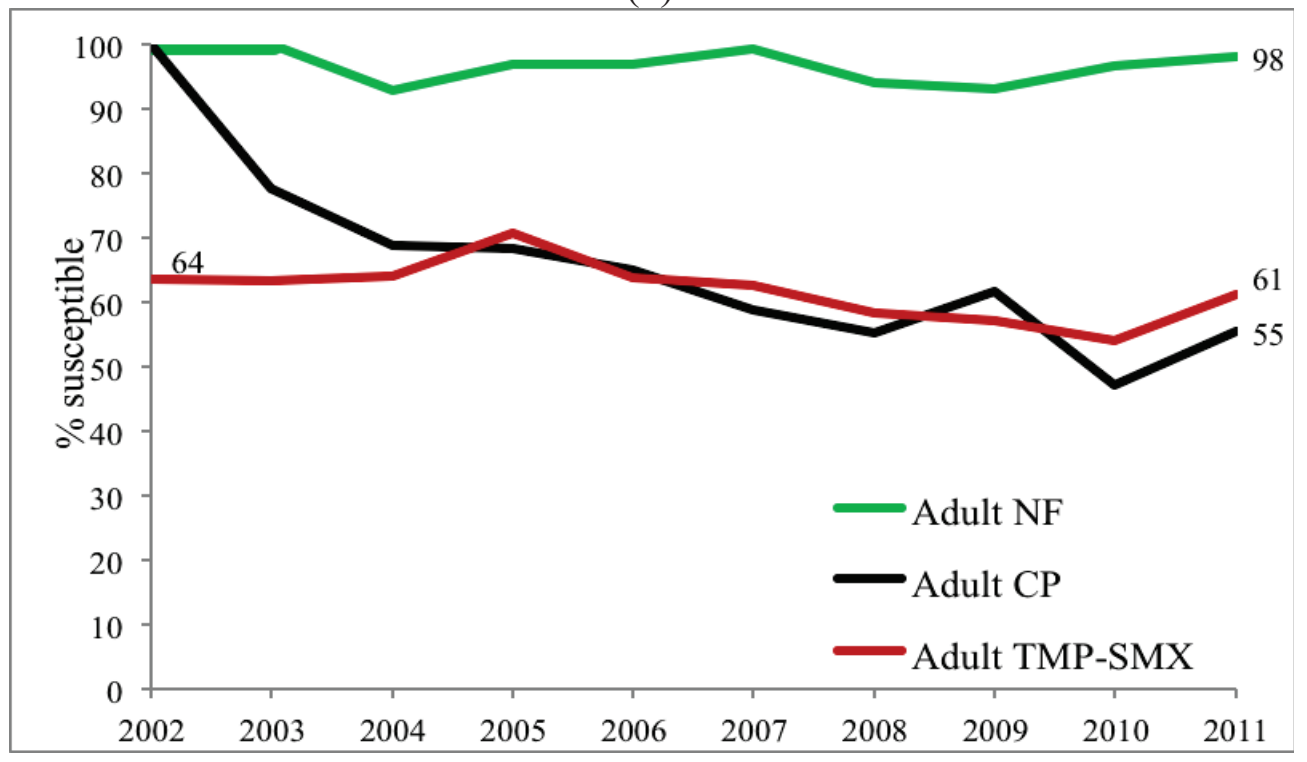

(b)

\section{Discussion}

Simultaneous to development of powerful and less toxic antimicrobial agents, infecting organisms are developing more resistance to antimicrobial agents. This has been a trend that has been reported over the last 20 years, as widespread usage of antimicrobial agents is believed to create selection pressure for more resistant organisms [1]. Case-control studies comparing children with and without TMP-SMX resistant organisms have shown that children who had antimicrobials 
agents for greater than four weeks in the previous six months were 23 times more likely to have resistance compared with those without resistance; children with genitourinary abnormalities were 2.4 times more likely to have resistance; and children with previous hospital admissions 2.3 times more likely [2] In other cases, specific clones of multidrug-resistant E. coli have been shown to behave with "epidemic outbreak" characteristics. The sources of these clonal epidemic-like outbreaks are unclear; some evidence implicates food and/or contact with food sources that had antimicrobial agent contact [3]. While international, national, regional, local, or hospital-wide antibiograms reflecting organism resistance attract attention [4,5], there is limited research on organism resistance and susceptibility diversity by practice site within institutions.

In this study we document that: (1) E. coli antimicrobial susceptibility differs widely between adults and children; (2) further variation occurs by practice site within a single medical center; and (3) differing drug trends in susceptibilities may occur. We hypothesize that these practice site susceptibility differences reflect the differing antimicrobial practice patterns of specialists and their specialty practice patterns. These antimicrobial drug trends may show: (a) differing susceptibilities between adults and children; (b) similar decreasing antimicrobial drug susceptibilities for both adults and children; and (c) persistently low susceptibilities for both. Our findings suggest that antimicrobial susceptibility and resistance patterns are affected by specialty practice patterns.

This study shows that pediatric and adult $E$. coli UTI susceptibility trends differ for three commonly used antimicrobial agents. With limited usage in children overall CP susceptibilities (69\%-88\%) are high in children except for the lower levels in ambulatory urology with higher usage; in contrast results show lower susceptibility in adults with lowest levels in adult ambulatory urology. A decreasing susceptibility rate for E. coli in urology practices has been noted by others in Japan [6]. The widespread usage of TMP-SMX in children for otitis media in addition to UTI is reflected in overall E. coli susceptibilities of $39 \%-62 \%$ while adult susceptibilities range from $61 \%-71 \%$. The stable higher E. coli susceptibilities of NF for both adults $(>92 \%)$ and children $(>95 \%)$ for the study periods most likely reflect relatively limited usage of NF for lower urinary tract infections and prophylaxis as reported by others [7]. By combining data from multiple institutional and internal practice sites, others have not revealed the range of $E$. coli susceptibilities within a single site nor the differences between children and adults [8]. Our data show that using individual hospital or institutional antibiograms to guide empiric antimicrobial treatment of a UTI in child or adult, moreover, may be misleading (Figures 1 and 2 and Tables 3 and 4).

These data and conclusions are limited by several considerations. First, prior to 2005 the sources of our pediatric specimens were not separated by outpatient and inpatient locations, so data trends are from 2005 forward, and second, CP patterns were not recorded in 2002 for technical reasons. Because data were de-identified, we could not eliminate repeat specimen data for patients. While multiple specimens from individuals could decrease or increase our susceptibility calculations, we believe that the large number of specimens involved reflect valid trends. Our usage of threshold of $10,000 \mathrm{CFU} / \mathrm{ml}$ as a positive culture may have excluded some urinary specimens with fewer resistant organisms, and this could overestimate our susceptibility reports. Finally, these studies compare data from an institution that offers primary medical care to the surrounding community in addition to regional and national tertiary care, thus these data may be unique based upon this patient population. 


\section{Experimental}

After Stanford University Medical Center Institutional Review Board approval was obtained for this study, we performed a retrospective review of all urine cultures submitted to Stanford University Medical Center (SUMC) Microbiology Laboratory from 2002 to 2011. Cultures were from Stanford University Hospitals and Clinics (SUH) reflecting specimens obtained primarily from adults, and from Lucile Packard Children's Hospital and Clinics (LPCH) from children and adult women seen in obstetrics clinic (OB) or admitted to labor and delivery (L\&D). Data included inpatient (INPT) and outpatient (OPT) urinary specimens obtained by all collection methods; repeat specimens per individual could not be excluded because data were de-identified. Microbiological data were obtained from two sources: (1) the SUMC clinical microbiology laboratory electronic database that contains urinary culture and susceptibility reports and annual hospital-wide antimicrobial susceptibility data; and (2) the STRIDE database (Stanford Translational Research Integrated Database Environment that compiles annual urinary culture specimen numbers, including positive and negative results. This is an NIH (National Institutes of Health) sponsored program that compiles clinical data for research use from SUMC. From the annual microbiology laboratory reports, hospital-wide trends were compiled for both the adult and pediatric hospitals ("antibiograms"). These antibiogram reports include cultures and susceptibilities from any type of culture (example, urine, blood, tracheal aspirates, etc.) processed at the SUMC laboratory.

STRIDE total urine cultures data were screened for selection using UTI diagnosis based on the following ICD-9 codes: 599.0 (UTI site not specified), 595 (cystitis), 590.1 (Acute pyelonephritis), 590.8 (Pyelonephritis unspecified). These data were segregated between the adult and pediatric hospitals by using an age cutoff of 18 years and below for pediatric patient data. This method generated total numbers of urinary cultures sent for populations. Antibiotic urinary susceptibility data was retrieved from the microbiology laboratory database that stores data for adult and pediatric hospitals separately. All SUMC clinical microbiology laboratory electronic urinary culture data were first parsed for overall antimicrobial susceptibility trends, then separated between INPT and OPT. The OPT clinical site data were further subdivided to various ambulatory clinics. For the adult hospital the OPT sites included the ED (emergency department), Gen Med (all internal medicine clinics including Stanford family medicine, Stanford medical group, Stanford family medicine, off-site community practice medical clinics using the Stanford laboratory), GYN (Gynecology) and URO (Urology). The pediatric OPT sites included the ED, Gen Peds (General pediatric clinics, including onsite and offsite community practice groups), OB (Obstetrics/Labor and delivery) and URO (Urology). Of note, except for the ED, prior to 2005, the pediatric hospital data entry did not code separately for outpatient specialty clinic sites.

Urinary specimens with $E$. coli were considered positive at a threshold of $10,000 \mathrm{cfu} / \mathrm{mL}$ (colony forming units per milliliter) in purity or predominance. This threshold was chosen because antimicrobial susceptibilities are routinely reported for $10,000 \mathrm{CFU} / \mathrm{mL}$ and we were examining E. coli susceptibilities in urinary cultures not urinary tract infection rates. Antimicrobial susceptibilities were performed using a Vitek2 microbial identification system (bioMérerieux Inc., Durham, NC, USA) and MICs were interpreted according to the Clinical and Laboratory Standard Institute M100 document. 


\section{Statistical Analysis}

We calculated the percentage annual urinary culture susceptibility as the numbers of specimens sensitive to the specific antibiotic divided by the annual E. coli positive cultures (CP, TMP-SMX and NF). The figures obtained were plotted annually to obtain the annual trend corresponding to the various clinical sites evaluated. Independent (unpaired) two-tailed Student's $t$-test ( $p$-value) were calculated for comparison of adult versus pediatric sites susceptibility to assess the statistical significance of differences seen among clinical sites in adults and children.

\section{Conclusions}

We report that antimicrobial susceptibilities for E. coli urinary cultures from a single institution may differ significantly between adults and children for three commonly used agents. Differences may occur, furthermore, among various practice sites within a single hospital site. We suggest that these differences in susceptibility reflect distinct specialty clinical prescribing practices. As a result of these variations, reliance upon any single regional or local antibiograms within large practice medical centers could be misleading.

\section{Author Contributions}

Winifred Owumi designed the study, performed data retrieval and analyses, and wrote portions of the paper. Niaz Banaei directed and reviewed data retrieval and reviewed the paper. Linda D. Shortliffe designed the study, directed analyses, and wrote and edited the paper.

\section{Conflicts of Interest}

The authors declare no conflict of interest.

\section{References}

1. Kunin, C.M. Resistance to antimicrobial drugs - A worldwide calamity. Ann. Int. Med. 1993, $118,557-561$.

2. Allen, U.D.; MacDonald, N.; Fuite, L.; Chan, F.; Stephens, D. Risk factors for resistance to “ first-line" antimicrobials among urinary tract isolates of Escherichia coli in children. Can. Med. Assoc. J. 1999, 160, 1436-1440.

3. Drexler, M. Secret Agents: The Menace of Emerging Infections; Joseph Henry Press: Washington, DC, USA, 2002.

4. Morrissey, I.; Hackel, M.; Badal, R.; Bouchillon, S.; Hawser, S.; Biedenbach, D. A review of ten years of the study for monitoring antimicrobial resistance trends (SMART) from 2002 to 2011. Pharmaceuticals 2013, 6, 1335-1346.

5. Bouchillon, S.K.; Badal, R.E.; Hoban, D.J.; Hawser, S.P. Antimicrobial susceptibility of inpatient urinary tract isolates of gram-negative bacilli in the United States: Results from the study for monitoring antimicrobial resistance trends (SMART) program: 2009-2011. Clin. Ther. 2013, 35, 872-877. 
6. Yamamichi, F.; Shigemura, K.; Matsumoto, M.; Nakano, Y.; Tanaka, K.; Arakawa, S.; Fujisawa, M. Relationship between urinary tract infection categorization and pathogens' antimicrobial susceptibilities. Urol. Int. 2012, 88, 198-208.

7. Kashanian, J.; Hakimian, P.; Blute, M., Jr.; Wong, J.; Khanna, H.; Wise, G.; Shabsigh, R. Nitrofurantoin: The return of an old friend in the wake of growing resistance. BJU Int. 2008, 102, 1634-1637.

8. McGregor, J.C.; Elman, M.R.; Bearden, D.T.; Smith, D.H. Sex- and age-specific trends in antibiotic resistance patterns of Escherichia coli urinary isolates from outpatients. BMC Fam. Pract. 2013, 14, e25. 


\title{
Chapter 6
}

\section{Antibiotic Prescribing in Primary Care and Antimicrobial Resistance in Patients Admitted to Hospital with Urinary Tract Infection: A Controlled Observational Pilot Study}

\author{
Ceire Costelloe, O. Martin Williams, Alan A. Montgomery, Colin Dayan \\ and Alastair D. Hay
}

\begin{abstract}
There is growing evidence that primary care prescribed antibiotics lead to antibiotic resistance in bacteria causing minor infections or being carried by asymptomatic adults, but little research to date has investigated links between primary care prescribed antibiotics and resistance among more serious infections requiring hospital care. Knowledge of these effects is likely to have a major influence on public expectations for, and primary care use of, antibiotics. This study aimed to assess the feasibility of recruiting symptomatic adult patients admitted to hospital with urinary infections and to link primary and secondary data information to investigate the relationship between primary care prescribed antibiotics and antimicrobial resistance in these patients. A microbiology database search of in patients who had submitted a urine sample identified 740 patients who were potentially eligible to take part in the study. Of these, 262 patients did not meet the eligibility criteria, mainly due to use of a urinary catheter (40\%). Two-hundred and forty three patients could not be recruited as the nurse was unable to visit the patients prior to discharge, as they were too unwell. Eighty patients provided complete information. Results indicate that there is evidence that prior antibiotic use is associated with resistant infections in hospital patients. A fully powered study, conducted using routinely collected data is proposed to fully clarify the precision of the association.
\end{abstract}

Reprinted from Antibiotics. Cite as: Costelloe, C.; Williams, O.M.; Montgomery, A.A.; Dayan, C.; Hay, A.D. Antibiotic Prescribing in Primary Care and Antimicrobial Resistance in Patients Admitted to Hospital with Urinary Tract Infection: A Controlled Observational Pilot Study. Antibiotics 2014, 3, 29-38.

\section{Introduction}

Resistance to antibiotics is a major threat to public health, and in the European Union, about 25,000 patients die in hospital each year from infections caused by selected multidrug-resistant bacteria and the associated costs are estimated at about 1.5 billion Euros per year [1]. Of significant concern, is the rate at which bacteria are becoming resistant, which is outstripping the rate at which new antibiotics are being developed [2]. General Practitioners (GPs) are responsible for $80 \%$ of all antibiotics prescribed to humans [3]. Patient expectations for antibiotics are a powerful determinant of prescribing [4] and for some GPs and patients, antibiotic resistance is seen only as a theoretical [4] or 
minimal [5] risk. The relationship between primary care antibiotic prescribing and resistance is complex and incompletely understood. Previous studies have established the relationship between the use of antibiotics in primary care and the asymptomatic carriage of resistant bacteria in adults [6-8] and children [9]. A more recent systematic review [10] found 18 studies showing consistent, temporal relationships between prescribing and resistance in bacteria causing relatively minor infections and in asymptomatic subjects. These studies contribute important observations to the existing literature that improve our understanding of how antibiotic resistance develops [11], they may or may not be sufficient to persuade clinicians [12] or patients [13] of the value of judicious antibiotic use in relation to reducing antibiotic resistance among patients with symptomatic infections, especially those requiring secondary care treatment.

Therefore, this paper reports a pilot and feasibility study, which aimed to investigate the relationship between primary care prescribed antibiotics and antimicrobial resistance, among symptomatic adult patients admitted to hospital with infection. Knowledge of these effects, and their associated economic impact, is likely to have a major influence on public expectations for, and primary care use of, antibiotics.

The recently published European Strategic Action Plan on Antimicrobial Resistance called for research using links between primary and secondary care to reduce morbidity and mortality associated with antimicrobial resistance, to promote prudent use of antibiotics and to raise awareness of the emergence and spread of antimicrobial resistance across all healthcare settings [14]. The study aimed to use routinely collected data stored within the patient primary and secondary care record, and a key component of the study was to examine the feasibility of linking this information at the individual patient level. Specifically our objectives were to: determine optimal recruitment, consent and data collection methods; describe the prevalence of antimicrobial susceptibility/resistance among isolated Escherichia coli (E. coli) from patients with urinary tract infection (UTI); and to examine primary care antibiotic prescribed within 12 months of admission and resistance to inform the sample size calculation of future studies.

\section{Results and Discussion}

Between October 2010 and July 2011, 6783 urine samples were submitted for microbiology testing at UHBristol Trust laboratory. Of these, 2147 grew an organism, of which 1407 (65\%) were ineligible (contaminant). The remaining 740 samples were from patients who were then potentially available to be screened for study eligibility (see Figure 1). Thirty-three female patients and twenty-five male patients could not be followed up due to lack of nurse time $(n=58)$. Thirty-eight percent of eligible patients were subsequently identified as ineligible and a further $36 \%$ could not meet with the research nurse as they were too unwell. Complete primary care data were available for 80 participants.

\subsection{Practices and Participants}

Patients were registered with a total of 25 GP practices with the majority of practices contributing one patient, and six practices having more than three patients recruited to the study. Mean (SD) participant age was 63.4 (20.1), with patient age ranging from 20-92 years. Sixty-four percent of 
the study cohort was female and $73 \%$ had been administered antibiotics in secondary care. The majority of these $(86 \%)$ were given penicillin and/or trimethoprim $(82 \%)$. Other characteristics of the study population are detailed in Table 1.

Figure 1. Diagram showing flow of participants through the study.

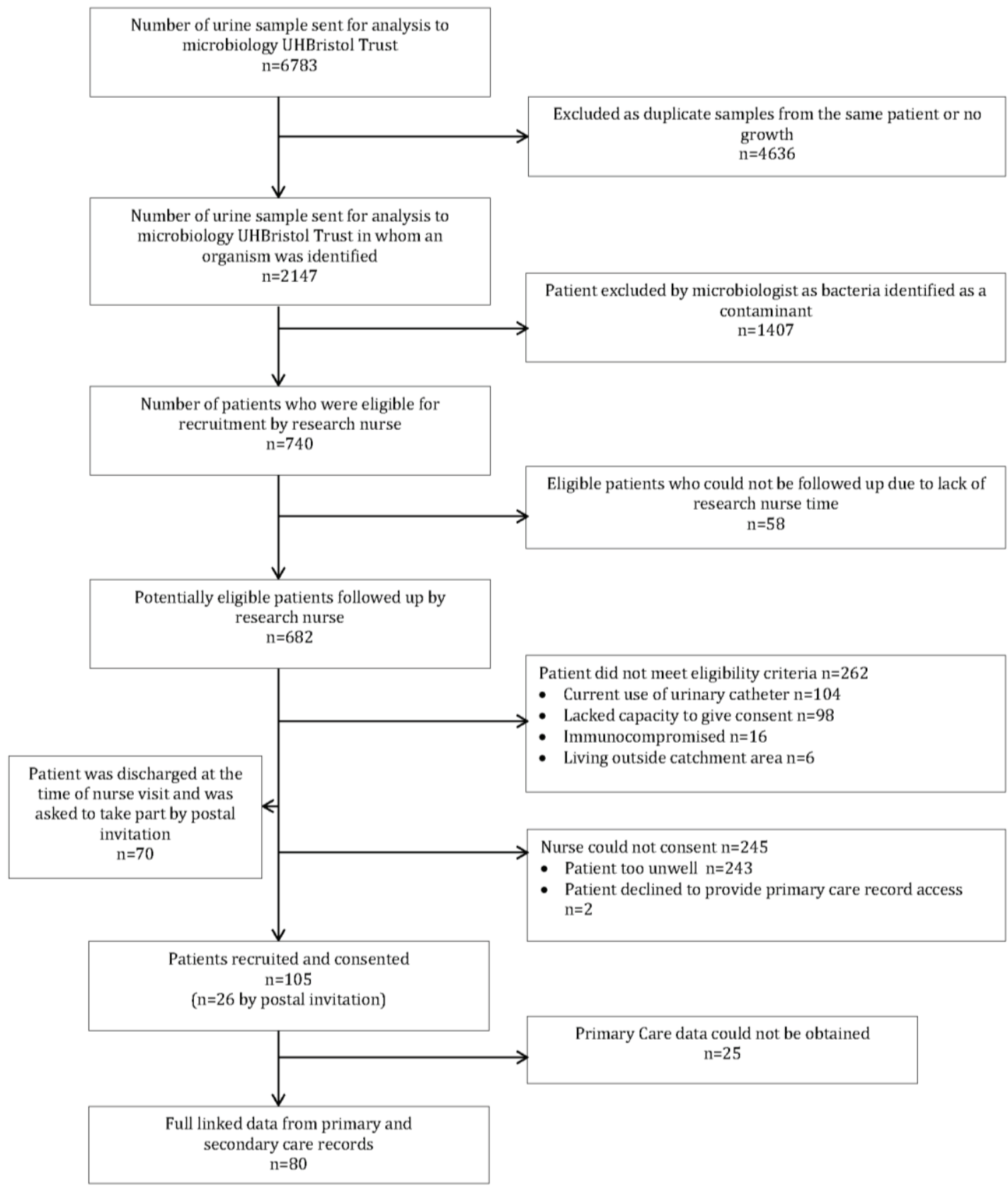

Table 2 details the microbiology results for 105 recruited patients. E. coli was the most common organism. Thirty-three and 24 percent of urinary isolates tested were resistant to trimethoprim and amoxicillin respectively. 
Table 1. Characteristics of patient participating in study.

\begin{tabular}{|c|c|}
\hline Variable & $n(\%) n=105$ \\
\hline Age (mean(SD)) & $63.4(20.1)$ \\
\hline Age category & - \\
\hline $20-49$ & $24(23)$ \\
\hline $50-74$ & $38(36)$ \\
\hline $75-84$ & $25(24)$ \\
\hline $85+$ & $18(17)$ \\
\hline Female & $67(64)$ \\
\hline Secondary care antibiotic use & $77(73)$ \\
\hline Trimethoprim $^{1}$ & $63(82)$ \\
\hline Co-amoxiclav ${ }^{1}$ & $22(29)$ \\
\hline Amoxicillin ${ }^{1}$ & $66(86)$ \\
\hline Nitrofurantoin ${ }^{1}$ & $3(4)$ \\
\hline Visited hospital in previous 12 months & $53(50)$ \\
\hline Antibiotic use in previous 12 months & - \\
\hline Yes & $21(20)$ \\
\hline Unknown $^{2}$ & $79(75)$ \\
\hline Co-morbidity & - \\
\hline Asthma & $14(13)$ \\
\hline Diabetes mellitus & $16(15)$ \\
\hline COPD & $3(3)$ \\
\hline History of smoking & $46(44)$ \\
\hline
\end{tabular}

Primary care data on antibiotic use was obtained for $63 \%(n=80)$ of the recruited sample. Table 3 details the number of antibiotic used by patients in the previous 12 months. Thirty-seven percent of participants had no antibiotic prescriptions in the previous 12 months. Twenty-three percent of participants had three or more course of antibiotics prescribed in the previous 12 months.

Unadjusted regression analysis showed that prescription of an antibiotic course in the previous 12 months was associated with presence of a urinary isolate resistant to trimethoprim (OR $3.5895 \%$ CI 1.18 to 10.9 , Table 4).

Table 2. Organisms identified in patient urine samples.

\begin{tabular}{cc}
\hline Organism identified & $\boldsymbol{n}(\mathbf{\%}), \boldsymbol{n}=\mathbf{1 0 5}$ \\
\hline Escherichia coli & $75(71)$ \\
Proteus spp. & $10(10)$ \\
Coliform & $20(19)$ \\
\hline Resistance (all urinary isolates) & $\boldsymbol{n}=\mathbf{1 0 5}$ \\
\hline Trimethoprim & $35(33)$ \\
Amoxicillin & $25(24)$ \\
Ciprofloxacin & $10(10)$ \\
\hline
\end{tabular}


Table 3. Primary care antibiotic courses in the previous 12 months.

\begin{tabular}{cc}
\hline Antibiotic type & $\boldsymbol{n ( \% )} \boldsymbol{n}=\mathbf{1 5 4}^{\mathbf{a}}$ \\
\hline Amoxicillin & $20(13)$ \\
Co-amoxiclav & $16(10)$ \\
Flucloxacillin & $13(8)$ \\
Nitrofurantoin & $28(18)$ \\
Trimethoprim & $31(20)$ \\
Ciprofloxacin & $17(11)$ \\
Erythromycin & $15(10)$ \\
Clarithromycin & $4(3)$ \\
Other & $10(7)$ \\
\hline Number of courses & $\boldsymbol{n}=\mathbf{8 0}$ \\
\hline 0 & $31(39)$ \\
1 & $22(27)$ \\
2 & $6(8)$ \\
$3-5$ & $12(15)$ \\
$6+$ & $9(11)$ \\
\hline
\end{tabular}

${ }^{a}$ Number of courses prescribed $n=154$ for $n=80$ patients. Twenty-seven patients had more than one course of antibiotic prescribed in the previous 12 months.

Table 4. Crude association between prescription of any antibiotic in the previous 12 months and trimethoprim resistance in patients admitted to hospital with suspected infection.

\begin{tabular}{cccc}
\hline $\begin{array}{c}\text { Antibiotic Prescribed } \\
\boldsymbol{n}=\mathbf{8 0}\end{array}$ & $\begin{array}{c}\text { Resistant } \\
\boldsymbol{n = 2 5 ( 3 1 \% )}\end{array}$ & $\begin{array}{c}\text { Susceptible } \\
\boldsymbol{n}=\mathbf{5 5}(\mathbf{6 9 \%})\end{array}$ & $\begin{array}{c}\text { Crude OR } \\
\mathbf{9 5 \%} \text { CI }\end{array}$ \\
\hline Yes 49(61) & $20(80 \%)$ & $29(53 \%)$ & 3.58 \\
No 31 (39) & $5(20 \%)$ & $26(47 \%)$ & 1.18 to 10.9 \\
\hline
\end{tabular}

\subsection{Discussion}

This study has demonstrated that recruitment of patients in secondary care is feasible, but requires significant staff resources. Data linkage methods, once patient consent had been obtained, was a successful method of carrying out this research. Primary Care data was obtained from the GP practices and was facilitated by local Primary Care Research network support. Using "opt-in" consent methods led to a high proportion $(36 \%)$ of potentially eligible patients not being given the opportunity to participate in the study, and incomplete data where patients provided insufficient information on their GP practice. This has negative outcomes not only for the generalizability of the study findings but, given that many of these patients were also too unwell to give consent, excludes patients suffering from more severe resistant infections. Furthermore, results of the pilot study showed that most patients were supportive of the research, with just two $(0.3 \%)$ potentially eligible participants declined to take part in the research, as they were concerned about giving access to their medical records. This suggests that the opt-in consent method may not be the most scientifically robust or efficient method. Given that most of the data used in this study are routinely 
collected in primary and secondary care, with appropriate data security safeguards and regulatory approvals, this research could be conducted without any direct patient involvement.

Results from a univariate analysis suggest that the primary care prescription of antibiotic in the 12 months prior to admission was associated with the presence of trimethoprim resistant isolates in patients admitted to hospital with UTI. This association appears clinically important, but needs to be adjusted for potential confounders (such as recent hospital admission) in future, more powerful, studies.

In addition, in any future study sample size should be sufficient to allow for comparison between resistance to specific antibiotics. An important factor, which was identified in this study, is that resistance testing is not standardized within the secondary care setting. A further limitation of this study is that the primary reason for admission was unknown, reflected in the variety of antibiotic therapies recorded. This information should be included in a future study. A large proportion of the study sample was excluded as they had a urinary catheter, which threatens the generalizability of study findings. In a future fully powered study, the sample size should allow for subgroup analysis - for example, investigating the association between primary care antibiotic and resistance in patients who have a catheter or patients who have co-morbidities.

Our pilot study produced results similar to those seen elsewhere. One Spanish study examined susceptibility to a number of antibiotics in patients treated by emergency services, who had been diagnosed with community acquired UTI. Patients who had been prescribed nalidixic acid or fluoroquinolones within the previous three months were found to have significantly higher resistance rates than those who had no previous primary care antibiotics [15]. A second study found that the odds of E. coli resistance to co-amoxiclav were four times higher comparing French patients hospitalized for urinary tract infection with and without co-amoxiclav exposure in the month prior to admission [16]. Finally, a cohort study retrospectively studied 24 adults with bacteraemic pneumonia (25 episodes) due to penicillin-resistant pneumococci compared with 48 patients with bacteraemic pneumonia caused by penicillin-sensitive pneumococci. The 24 patients with penicillin-resistant pneumococci had a significantly higher: incidence beta-lactam antibiotic use during the previous three months, hospitalization during the previous three months, nosocomial pneumonia and episodes of pneumonia during the previous year [17]. However, these studies are all relatively small and resistance rates vary substantially across Europe with Spain having higher rates than typically seen in the UK.

\subsection{Implications for Research and Clinical Practice}

Since much of the study data collected could have been collected from the microbiology database and the patient's primary and secondary care records, database linkage methods might be more efficient for future studies. This would negate the need to recruit acutely unwell patients in the secondary care setting. Results from the study suggest that the decision to prescribe antibiotics in primary care can influence antibiotic resistance status in patients when they are admitted to secondary care, but needs to be confirmed in a fully adjusted, powered study. Further knowledge of association between primary care antibiotic prescribing and the development of resistant infections at an individual level will better inform shared GP-patient decision making in fully weighing up the pros and cons of prescribing and using antibiotics. This will benefit patients by discouraging the 
inappropriate prescription of antibiotics and help encourage the virtuous cycle of reduced antibiotic use and reduced bacterial resistance.

\section{Experimental}

\subsection{Design, Setting and Participants}

The study was a controlled observational pilot study including adult patients being treated for a microbiologically confirmed urinary tract infection (UTI) at the University Hospital Bristol Trust.

\subsection{Identification of Bacteria}

The microbiology database was searched daily for samples submitted within $48 \mathrm{~h}$ of admission in which a bacterium was isolated. To be included urines had to have both: a significant positive culture and either a clinical descriptor for a urinary tract infection (cystitis or pyelonephritis) or positive ward-based urine dip stick result. In the laboratory, a positive leukocyte esterase, and a significant growth (104-105 or $>105 \mathrm{cfu} / \mathrm{mL})$ of a single or two organisms would be considered significant. Three or more organisms were regarded as contaminants and were excluded.

\subsection{Microbiology Methods}

Bacterial identification was based on appearance in chromogenic agar or by using the automated VITEK II system. Standard British Society for Antimicrobial Chemotherapy (BSAC) [18] disc testing methods were used to determine antibiotic resistance status for any bacterium isolated from patient sample within the UHBristol Trust laboratories. Disc testing methods were used to detect resistance to a variety of antibiotics. As we used data produced from standard NHS procedures not all samples were tested for resistance to the same antibiotics.

\subsection{Patient Recruitment and Consent}

The hospital's microbiology database was searched to ascertain the location (which hospital ward or discharged) of patients in whom bacteria had been identified. The Research Nurse located each potentially eligible patient and asked permission from the patients' clinical team to approach the patient to provide information about the study. Patients were asked to give consent for their primary and secondary care medical records to be used to extract study data. In addition, patients were asked to complete a brief baseline questionnaire giving details of: hospital visits in the previous 12 months; antibiotic use in the previous 12 months; co-morbidities; current medication and socio-demographic information. If patients had been discharged before the nurse had an opportunity to recruit then an invitation letter and study material was sent from the microbiology team to the patient by post. Patients were ineligible if they had previously taken part in the study or had insufficient capacity to give informed consent, or if the sample had been obtained from a urinary catheter. If patients were: under 18 years of age; immunocompromised or unable to read and understand English; they were also excluded Ethical approval was given to search the primary care notes of patients from Bristol, North Somerset or South Gloucester Primary Care Trusts regions. Therefore, patients who were outside that catchment area were excluded. 


\subsection{Primary Care Antibiotic Data}

GPs were informed by letter of their patient's participation in the study. GPs were asked to complete a proforma to collect computer recorded primary care antibiotic prescribing and consultations in the 12 months prior to admission and six weeks post hospital discharge. In particular, the type, the number of units (capsules, tablets or suspension volume) per dose, the strength per unit, the total number of units and the dosing instructions was be collected for all courses of prescribed systemic antibiotics.

\subsection{Outcome Measures}

The main outcome of the study was to determine optimal recruitment, consent and data collection methods. In addition, we aimed to describe the prevalence of antimicrobial susceptibility/resistance among bacteria isolated from urine samples.

Summary statistics were used to describe recruitment and data attrition rates and demographics, antibiotic exposure and outcomes of individuals with susceptible and resistant bacteria. A separate binary variable was created to represent if an organism showed resistance to trimethoprim (Yes/No). Prior exposure to antibiotics in the 12 months before admission was recorded as a binary variable (Yes/No).

The pilot study was designed to estimate crude strengths of association to inform the sample size calculation of a future, definitive study. A logistic regression model was used to estimate the unadjusted strength of association between prior primary care antibiotic exposure and antibiotic resistance in the study and measures of variability between antibiotic exposure and resistance [19].

\section{Conclusions}

Our study has demonstrated an important possible relationship between primary care prescribed antibiotics and trimethoprim resistance in patients admitted to secondary care with UTI. This should be replicated in a fully powered study. We have also demonstrated that the recruitment methods are feasible, but that the study could be more efficiently conducted using routinely collected data. These data could be easily available for research purposes, enabling ongoing monitoring of resistant infections in hospitals. Improvements in database connections between healthcare settings could enable this information to be fed between primary care and secondary care clinicians - thereby leading to more appropriate antibiotic use both within primary and secondary health care settings.

\section{Acknowledgments}

We wish to thank the participating patients in the United Hospital Bristol Trust and practices in Bristol, North Somerset and South Gloucester. We wish to thank Dave Wright for advice and support in designing the database search and Angel Pollard and Susan Bryant for support with data collection.

This work was supported by the National Institute for Health Research, National School for Primary Care Research.

Ethics approval was granted by NRES Committee South West-Southmead. The reference number is $10 / \mathrm{H} 0102 / 3$. 


\section{Author Contributions}

$\mathrm{ADH}$ and $\mathrm{CC}$ conceived idea for project. $\mathrm{CD}$ advised on study design and patient recruitment. OMW carried out data extraction and screening of eligible samples. Research Nurse carried out all data collection. CC and AAM conducted data analysis. CC and ADH produced first draft of paper. OMW, CD and AAM commented on subsequent versions.

\section{Conflicts of Interest}

The authors declare no conflict of interest.

\section{References}

1. Leung, E.; Weil, D.E.; Raviglione, M.; Nakatani, H. The WHO policy package to combat antimicrobial resistance. Bull. World Health Organ. 2011, 89, 390-392.

2. Infectious Diseases Society of America. Bad Bugs, no Drugs: As Antibiotic Discovery Stagnate and A Public Health Crisis Brews; Infectious Diseases Society of America: Alexandria, VA, USA, 2004.

3. Standing Medical Advisory Committee. The Path of Least Resistance; Department of Health: London, UK, 1998.

4. Macfarlane, J.; Holmes, W.; Macfarlane, R.; Britten, N. Influence of patients' expectations on antibiotic management of acute lower respiratory tract illness in general practice: Questionnaire study. BMJ 1997, 315, 1211-1214.

5. Simpson, S.A.; Wood, F.; Butler, C.C. General practitioners' perceptions of antimicrobial resistance: A qualitative study. J. Antimicrob. Chemother. 2007, 59, 292-296.

6. Hay, A.D.; Thomas, M.; Montgomery, A.; Wetherell, M.; Lovering, A.; McNulty, C.; Lewis, D.; Carron, B.; Henderson, E.; MacGowan, A. The relationship between primary care antibiotic prescribing and bacterial resistance in adults in the community: A controlled observational study using individual patient data. J. Antimicrob. Chemother. 2005, 56, 146-153.

7. Malhotra-Kumar, S.; Lammens, C.; Coenen, S.; van Herck, K.; Goossens, H. Effect of azithromycin and clarithromycin therapy on pharyngeal carriage of macrolide-resistant streptococci in healthy volunteers: A randomised, double-blind, placebo-controlled study. Lancet 2007, 369, 482-490.

8. Costelloe, C.; Lovering, A.; Montgomery, A.; Lewis, D.; McNulty, C.; Hay, A.D. Effect of antibiotic prescribing in primary care on meticillin-resistant Staphylococcus aureus carriage in community-resident adults: A controlled observational study. Int. J. Antimicrob. Agents 2011, 39, 135-141.

9. Chung, A.; Perera, R.; Brueggemann, A.B.; Elamin, A.E.; Harnden, A.; Mayon-White, R.; Smith, S.; Crook, D.W.; Mant, D. Effect of antibiotic prescribing on antibiotic resistance in individual children in primary care: Prospective cohort study. Br. Med. J. 2007, 335, 429-434.

10. Costelloe, C.; Metcalfe, C.; Lovering, A.; Mant, D.; Hay, A.D. Effect of antibiotic prescribing in primary care on antimicrobial resistance in individual patients: Systematic review and meta-analysis. Br. Med. J. 2010, 340, c2096. 
11. Lipsitch, M.; Samore, M.H. Antimicrobial use and antimicrobial resistance: A population perspective. Emerg. Infect. Dis. 2002, 8, 347-354.

12. Butler, C.C.; Rollnick, S.; Pill, R.; Maggs-Rapport, F.; Stott, N. Understanding the culture of prescribing: Qualitative study of general practitioners' and patients' perceptions of antibiotics for sore throats. Br. Med. J. 1998, 317, 637-642.

13. Brooks, L.; Shaw, A.; Sharp, D.; Hay, A.D. Towards a better understanding of patients' perspectives of antibiotic resistance and MRSA: A qualitative study. Fam. Pract. 2008, 25, 341-348.

14. World Health Organisation. European Strategic Action Plan on Antibiotic Resistance; Copenhagen, Denmark, 2011.

15. Alós, J.-I.; Serrano, M.G.; Gómez-Garcés, J.-L.; Perianes, J. Antibiotic resistance of Escherichia coli from community-acquired urinary tract infections in relation to demographic and clinical data. Clin. Microbiol. Infect. 2005, 11, 199-203.

16. Leflon-Guibout, V.; Ternat, G.; Heym, B.; Nicolas-Chanoine, M.H. Exposure to co-amoxiclav as a risk factor for co-amoxiclav-resistant Escherichia coli urinary tract infection. J. Antimicrob. Chemother. 2002, 49, 367-371.

17. Pallares, R.; Gudiol, F.; Linares, J.; Ariza, J.; Rufi, G.; Murgui, L.; Dorca, J.; Viladrich, P.F. Risk factors and response to antibiotic therapy in adults with bacteremic pneumonia caused by penicillin-resistant pneumococci. N. Engl. J. Med. 1987, 317, 18-22.

18. BSAC Microbiology Testing Guidelines. Available online: http://bsac.org.uk/wp-content/ uploads/2012/02/version215-nov-2003-.pdf (accessed on 1 October 2010).

19. Arain, M.; Campbell, M.J.; Cooper, C.L.; Lancaster, G.A. What is a pilot or feasibility study? A review of current practice and editorial policy. BMC Med. Res. Methodol. 2010, 10, e67. 


\title{
Chapter 7
}

\section{Value of Provoked or Spontaneous Flank Pain in Men with Febrile Urinary Tract Infections}

\author{
Franck Bruyère, Joseph-Alain Ruimy, Louis Bernard, Raphael Elfassi, Olivier Boyer, \\ Fabrice Amann and Paul Meria
}

\begin{abstract}
Background: Our objective was to identify the clinical, laboratory and radiological characteristics of febrile urinary tract infections (UTI) in men and to focus on the value of flank pain in these men managed in an ambulatory care system. Methods: A network was designed to manage men with febrile UTI without hospitalization according to an algorithm designed with different specialists. The patients' characteristics were prospectively recorded and each patient was followed up until completely cured. We artificially divided patients into two groups. Group 1: men without flank pain diagnosed as prostatitis and a second group (Group 2) of men with flank pain or provoked flank pain more likely to have a pyelonephritis. Groups were compared to find arguments to differentiate prostatitis to pyelonephritis. Results: 350 men were included in the study, half of these men reported urinary symptoms (dysuria, urgency and burning urination). The negative predictive values of the nitrite and leukocytes test were poor alone or in combination. The renal ultrasound was never informative. None of the patients failed to respond to the treatment. No difference was found between groups. Conclusions: Laboratory test results and radiological features had a poor predictive value. Men with suspected pyelonephritis did not evolve differently from those with suspected prostatitis. Monitoring and treatment of men with febrile UTI does not seem to depend on the existence of a pyelonephritis suspected after the presence of a lumbar pain. Ambulatory management of febrile UTI is feasible and safe, requiring an efficient network for patient's surveillance.
\end{abstract}

Reprinted from Antibiotics. Cite as: Bruyère, F.; Ruimy, J.-A.; Bernard, L.; Elfassi, R.; Boyer, O.; Amann, F.; Meria, P. Value of Provoked or Spontaneous Flank Pain in Men with Febrile Urinary Tract Infections. Antibiotics 2014, 3, 155-162.

\section{Introduction}

Acute genitourinary tract infections such as acute prostatitis (AP) represent a significant problem in urological practice. Unfortunately, very few data have been published regarding this subject. In the U.S., AP is the most common urological diagnosis in men under 50 years of age, representing $8 \%$ of consultations [1]. Antibiotic treatment of AP is recommended, but abscess formation has been described, which may have devastating consequences. It is now acknowledged that the vast majority of febrile urinary infections in men are to be considered as acute prostatitis; pyelonephritis remains exceptional in men. Flank pain, spontaneous or provoked, is the clinical sign that orients the diagnosis toward a pyelonephritis. Unfortunately, no pathognomonic clinical, 
biological or radiological signs prove the diagnosis. We analyzed 251 cases of febrile urinary infections in men treated in a medical network; all of the data were prospectively recorded by a data manager and were analyzed retrospectively.

\section{Methods}

A network was designed to include general practitioners (GP), private biological departments and private radiological departments to manage febrile urinary infections without hospitalization. Each patient was managed "at home" by scheduling clinical examinations, laboratory tests and imaging using the network. All of the GPs involved in the group were educated on how to use an algorithm initially built with specialists and were asked to enter information into a database. Treatments were defined as successful if symptoms disappeared in less than $72 \mathrm{~h}$ and if no recurrence was observed within one month. Herein, we present the entire clinical, laboratory, radiological and bacteriological data for men with acute febrile urinary infection treated between May 2005 and December 2012 as part of the SphereS. We analyzed 350 men who presented with febrile urinary infection. The data were collected prospectively in a customized database and were retrospectively analyzed. We included all men who were recruited by their GP because of suspicion of a febrile urinary infection (fever and urinary symptoms); however, men with suspected urethritis (urethral flow and pain without fever) were excluded from the study. Once enrolled in the study, none of the men were excluded from the final analysis. We artificially divided patients into two groups. Group 1: men without flank pain diagnosed as prostatitis and a second group (Group 2) of men with flank pain or provoked flank pain more likely to have a pyelonephritis. Groups were compared to find arguments to differentiate prostatitis to pyelonephritis.

\section{Statistical Analysis}

Continuous variables were reported as the mean value plus or minus the standard deviation. The sensitivity, specificity and positive and negative predictive values were calculated for several variables. We used chi-square analysis and alternatively, when indicated, Fisher's exact test and Wilcoxon for non-parametric comparison of means. The significance of the difference between groups was estimated by way of a two-tailed Student's $t$-test.

\section{Results and Discussion}

Three hundred and fifty men were enrolled in the study. The patient characteristics are summarized in Table 1.

All men were referred to their GP because of suspicion of febrile acute urinary tract infection. All men were referred because of fever higher than $38{ }^{\circ} \mathrm{C}$, and half of these men reported urinary symptoms (dysuria, urgency and burning urination).

A total of $289\left(82.6 \%\right.$ ) patients had neutrophil counts exceeding $8000 / \mathrm{mm}^{3}$ (Table 2). 
Table 1. Patient characteristics of 350 men with febrile urinary infection. Group 1: no flank pain; group 2: provoked or spontaneous flank pain.

\begin{tabular}{ccccc}
\hline characteristics & Total & Group 1 $(\boldsymbol{n}=\mathbf{3 0 8})$ & Group 2 $(\boldsymbol{n}=\mathbf{4 2})$ & $\boldsymbol{p}$ value \\
\hline Mean age \pm SD years & $60.2 \pm 16.2$ & $61.0 \pm 15.8$ & $54.8 \pm 18.2$ & 0.04 \\
History of UTI (\%) & $82(23.4 \%)$ & $79(25.6 \%)$ & $3(7.1 \%)$ & 0.02 \\
Diabetes (\%) & $22(6.3 \%)$ & $18(5.8 \%)$ & $4(9.5 \%)$ & $\mathrm{ns}$ \\
Known prostate enlargement (\%) & $37(10.5 \%)$ & $36(11.6 \%)$ & $1(2.3 \%)$ & $\mathrm{ns}$ \\
Flank pain & $18(5.1 \%)$ & $0(\%)$ & $18(42.8 \%)$ & \\
Provoked flank pain & $39(11.1 \%)$ & $0(0 \%)$ & $39(92.8 \%)$ & \\
Macroscopic hematuria & $71(20.3 \%)$ & $62(20.1 \%)$ & $6(21.4 \%)$ & $\mathrm{ns}$ \\
Flu-like symptoms & $29(8.3 \%)$ & $24(7.8 \%)$ & $5(11.9 \%)$ & $\mathrm{ns}$ \\
Urinary bother & $178(50.8 \%)$ & $159(51.6 \%)$ & $19(45.3 \%)$ & $\mathrm{ns}$ \\
painful DRE & $15(4.3 \%)$ & $14(4.5 \%)$ & $1(2.3 \%)$ & $\mathrm{ns}$ \\
\hline
\end{tabular}

Abbreviations: SD, standard deviation; UTI, urinary tract infection; DRE, digital rectal examination; ns, non significant.

Table 2. Laboratory test results for men with acute urinary infection.

\begin{tabular}{ccccc}
\hline Laboratory test & Total & Group 1 $(\boldsymbol{n}=\mathbf{3 0 8})$ & Group 2 $(\boldsymbol{n}=\mathbf{4 2})$ & $\boldsymbol{p}$ value \\
\hline Leukocytes $\left(10^{3} / \mathrm{mm}^{3}\right)$ & $14.4 \pm 4.9$ & $14.7 \pm 5.0$ & $14.2 \pm 4.3$ & $\mathrm{~ns}$ \\
\hline Neutrophils $\left(10^{3} / \mathrm{mm}^{3}\right)$ & $12.1 \pm 4.6$ & $12.2 \pm 4.6$ & $11.6 \pm 4.1$ & $\mathrm{~ns}$ \\
Creatinine $(\mu \mathrm{mol} / \mathrm{L})$ & $102 \pm 24$ & $102 \pm 25$ & $97 \pm 20$ & $\mathrm{~ns}$ \\
Creatinine clearance $(\mathrm{mL} / \mathrm{min})$ & $85 \pm 25$ & $84 \pm 24$ & $95 \pm 32$ & $\mathrm{~ns}$ \\
C reactive protein $(\mathrm{mg} / \mathrm{L})$ & $100 \pm 83$ & $99 \pm 83$ & $104 \pm 83$ & $\mathrm{~ns}$ \\
Urinary leukocytes $(\mathrm{mean} \pm \mathrm{SD}) / \mathrm{mm}^{3}$ & $525 \pm 820$ & $551 \pm 860$ & $337 \pm 379$ & 0.01 \\
Urinary red blood cells & $143 \pm 312$ & $142 \pm 302$ & $152 \pm 385$ & $\mathrm{~ns}$ \\
Positive urinary bacterial culture & $298(85.1 \%)$ & $268(87.0 \%)$ & $30(71.4 \%)$ & $\mathrm{ns}$ \\
Bacteriological findings & & & & \\
Escherichia coli & $247(82.8 \%)$ & $230(85.8 \%)$ & $17(56.6 \%)$ & \\
Proteus & $17(5.7 \%)$ & $17(6.3 \%)$ & $0(0.0 \%)$ & \\
Klebsiella & $8(2.6 \%)$ & $6(2.2 \%)$ & $2(6.6 \%)$ & \\
Citrobacter & $9(3.0 \%)$ & $8(3.0 \%)$ & $1(3.3 \%)$ & 0.04 \\
Enterococcus & $5(1.6 \%)$ & $4(1.5 \%)$ & $1(3.3 \%)$ & \\
Enterobacter & $4(1.3 \%)$ & $4(1.5 \%)$ & $0(0.0 \%)$ & \\
Morganella & $4(1.3 \%)$ & $3(1.1 \%)$ & $1(3.3 \%)$ & \\
Staphylococcus sp. & $3(1.0 \%)$ & $3(1.1 \%)$ & $0(0.0 \%)$ & \\
Haemophilus & $1(0.3 \%)$ & $0(0.0 \%)$ & $1(3.3 \%)$ & \\
\hline
\end{tabular}

Abbreviations: ns, non significant.

No difference was found between groups in terms of urine dipstick results. Among patients with urine dipsticks showing negative for leukocytes and nitrite, only $5.5 \%$ had a negative urine culture, $82.8 \%$ tested positive for E. coli, and 5.7\% tested positive for Proteus mirabilis. The sensitivity of the nitrite test was $67 \%$, and the specificity was $57 \%$; the sensitivity of the leukocyte test was $94.3 \%$, and the specificity was $5.8 \%$. The addition of the of the nitrite test results marginally improved the sensibility of the leukocyte test; however, the specificity remained low. The positive predictive value of a positive nitrite test and of the combination of a positive nitrite and a positive 
leukocyte test were respectively $90.1 \%$ and $86.1 \%$. The negative predictive values of the nitrite and leukocytes test were poor alone or in combination.

The urine dipstick was positive for leukocytes and nitrite in $97.9 \%$ of patients and was negative for both in $2.1 \%$ of the cases of positive urine culture (Table 3 ).

Table 3. Results of the urine dipstick test.

\begin{tabular}{ccc}
\hline \multirow{2}{*}{$\begin{array}{c}\text { Diagnostic test, result } \\
\text { (no. of patients with results) }\end{array}$} & \begin{tabular}{c} 
Culture result, no. (\%) \\
\cline { 2 - 3 }$(n=298)$
\end{tabular} & $\begin{array}{c}\text { negative } \\
(n=52)\end{array}$ \\
\hline Nitrite & & \\
positive (223) & $201(67.4)$ & $22(42.3)$ \\
negative (127) & $97(32.5)$ & $30(57.6)$ \\
\hline Leukocytes & & $49(94.2)$ \\
positive (330) & $281(94.3)$ & $3(5.8)$ \\
negative (20) & $17(5.7)$ & $47(90.7)$ \\
Nitrite, positive & & $5(9.6)$ \\
Leukocytes positive (339) & $292(97.9)$ & $49(94.2)$ \\
leukocytes negative (11) & $6(2.1)$ & $3(5.8)$ \\
\hline Nitrite, negative & & \\
Leukocytes positive $(296)$ & $247(82.9)$ & \\
Leukocytes negative (54) & $51(17.1)$ & \\
\hline
\end{tabular}

Twenty four patients $(6.8 \%)$ had a postvoiding volume greater than $80 \mathrm{~mL}$. In the remaining cases, the renal ultrasound was not informative (Table 4).

Table 4. Ultrasound findings.

\begin{tabular}{ccccc}
\hline & Total $(\boldsymbol{n}=\mathbf{3 5 0})$ & Group 1 $(\boldsymbol{n}=\mathbf{3 0 8})$ & Group 2 $(\boldsymbol{n}=\mathbf{4 2})$ & $\boldsymbol{p}$ Value \\
\hline Prostate volume mL (mean $\pm \mathrm{SD})$ & $43 \pm 23$ & $43 \pm 21$ & $38 \pm 34$ & $\mathrm{~ns}$ \\
Post voiding urinary volume & $46 \pm 112$ & $47 \pm 116$ & $30 \pm 54$ & $\mathrm{~ns}$ \\
Other comments: & & & & \\
Prostatic calcifications & $17(50.1 \%)$ & $16(55 \%)$ & $1(25 \%)$ & $\mathrm{ns}$ \\
Epididymitis & $2(5.9 \%)$ & $1(3 \%)$ & $1(25 \%)$ & $\mathrm{ns}$ \\
Prostate nodule & $1(2.9 \%)$ & $1(3 \%)$ & 0 & $\mathrm{~ns}$ \\
Bladder tumor & $2(5.8 \%)$ & $2(7 \%)$ & 0 & $\mathrm{~ns}$ \\
Prostatitis & $12(35.1 \%)$ & $10(31 \%)$ & $2(50 \%)$ & $\mathrm{ns}$ \\
Comments on kidney $(n=204)$ & & & & \\
Angioma & $1(0.4 \%)$ & $1(0.5 \%)$ & $0(0 \%)$ & $\mathrm{ns}$ \\
Stone & $7(3.4 \%)$ & $4(2.2 \%)$ & $3(11.5 \%)$ & 0.011 \\
Dilatation & $2(0.8 \%)$ & $1(0.5 \%)$ & $1(3.8 \%)$ & $\mathrm{ns}$ \\
Pyelonephritis & $1(0.4 \%)$ & $0(0 \%)$ & $1(3.8 \%)$ & $\mathrm{ns}$ \\
Normal & $193(94.6 \%)$ & $172(96.6 \%)$ & $21(80.8 \%)$ & $\mathrm{ns}$ \\
\hline
\end{tabular}

Abbreviations: SD, standard deviation.

Two cases of renal dilation were found; however, only one patient exhibited pain in his flank. Stones were more often found in patients with spontaneous or provoked flank pain $(p=0.011)$. 
Overall, $92.8 \%$ of patients were treated with fluoroquinolones. The mean duration of treatment was $22.9 \pm 6.6$ days $(18.9 \pm 7.1$ days for group 1 and $23.5 \pm 6.3$ days for group $2, p<0.001)$. None of the patients failed to respond to the treatment. Men returned with apyrexia in mean 2.7 days \pm 1.6 days. Only three men (one in group 1 and two in group 2) required hospitalization during follow up. No difference was found between groups. Men with suspected pyelonephritis did not evolve differently from those with suspected prostatitis. Monitoring and treatment of men with febrile urinary tract infection does not seem to depend on the existence of a suspected pyelonephritis suspected after the presence of a lumbar pain.

\section{Discussion}

Herein, we present one of the largest studies published on febrile urinary infection in men. Clinical, laboratory and radiological data were studied, and we report many useful concepts for the diagnosis and treatment of acute prostatitis and acute pyelonephritis.

The clinical characteristics of acute prostatitis are poorly described in the published literature [2,3]. The majority of the literature focuses on post-biopsy prostatitis [4-6]. Flank pain is frequently described (from $5.1 \%$ in our study to $25 \%$, two without any pyelonephritis), and prostatitis can be misdiagnosed initially as flank pain is interpreted as a clinical sign of pyelonephritis. Patients frequently describe urinary symptoms; however, the reported incidence for urinary symptoms is influenced by the specialty of the practitioner [3]. A total of $86 \%$ of patients questioned by a urologist for an acute prostatitis had urinary symptoms, while only $50 \%$ reported urinary symptoms if seen in geriatrics or internal medicine [3]. However, the population admitted to urology departments may have a higher incidence of bladder disorders than populations handled by other specialties. Only $63 \%$ of patients with AP in geriatric departments suffer from fever versus more than $80 \%$ in other departments ( $100 \%$ in our study). We could argue that the diagnosis of AP was suggested by geriatricians because of positive bacterial cultures in men with indwelling catheters. DREs suggesting AP are not routinely described (39\% for infectious disease specialists to $77 \%$ for urologists) [3]. In our study, only $4.4 \%$ of DRE were painful. DRE were not routinely performed by GPs in our study, as the initial algorithm found that the predictive value of DRE in acute situations strongly suggest AP to be poor. Unfortunately, we did not focus on past history of urinary tract infection (UTI). Etienne et al. reported that approximately $37 \%$ of men with AP report previous UTI [3]. In our study we found that patients with flank pain had fewer previous episodes of urinary tract infection without arguing for pyelonephritis or prostatitis.

$\mathrm{C}$ reactive protein (CRP) and leukocyte levels were elevated in $96 \%$ and $74 \%$ of men with AP, respectively [2]. We found similar results that did not correlate with the symptoms or the severity of UTI and without difference between AP or pyelonephritis. Analyzing these results, we suggest that the predictive value of CRP and leukocyte levels is low in the diagnosis of UTI; its value should be more precisely determined.

Blood cultures were not available in our study. In a previously published study, blood cultures were analyzed for acute prostatitis patients [7]. Blood cultures were positive for $21 \%$ of men and contributed to the microbiological diagnosis for $5 \%$ of the patients. Guidelines are not trivial for that purpose; these data suggest that cases of uncomplicated UTI, AP or pyelonephritis would not 
benefit from routine blood cultures. However, urine cultures are recommended, and the bacterial results are similar between studies.

In a previous study, urine dipsticks were used to test 422 men with urinary infections [8]. The authors concluded that the positive predictive value of a positive nitrite test was $96 \%$ and that the addition of the results of the leukocyte esterase test did not improve the diagnostic accuracy of the nitrite test. We agree with these conclusions, and we would like to emphasize the poor negative predictive value of urine dipsticks in men UTIs.

Post-voiding residual volumes were not different between groups in our study. In the Etienne study, only $14 \%$ of patients followed by an infectious disease specialist had post-voiding residual volume measurements done, and $21 \%$ of patients had postvoiding residual volume measurements done if followed by a urologist [3]. This result was not precise in Auzanneau's study, in which 49\% had a renal or bladder ultrasound [2]. We did not find any additional value of performing a renal or a prostatic ultrasound, except for stone diagnosis in men with flank pain. Based on all of these findings, we can argue that the only imaging technique that should be performed on men with suspected AP is a bladder ultrasound to measure the postvoiding residual volume and a renal ultrasound if men complain from spontaneous or provoked flank pains. The algorithm used informed us to perform prostate ultrasound if the patient remains febrile at day 3 after antibiotics in order to diagnose an abscess. None of the patients needed prostate ultrasound.

To our knowledge, this is one of the largest studies analyzing a substantial amount of data in an important population of men with febrile UTI. Data were collected prospectively and analyzed retrospectively. The network increases the follow up of patients with routine phone calls and specialist visits if the evolution of UTI is unfavorable or if the patient experiences recurrent episodes. The data examined in this study emphasized that the laboratory tests or radiological exams should be performed accurately. The national and international infectious disease committee should take into account these results to provide new recommendations for the diagnosis and treatment of men UTIs. The question about the diffenciation between AP and prostatitis remains unsolved. We can argue that no difference was found between men with flank pain and men without flank pain in term of clinical or biological data. Stones were, however, more frequently found on ultrasound if men complain of flank pain.

\section{Conclusions}

This study, conducted using data from patients with febrile UTI, revealed that the most common clinical features are fever with urinary discomfort. No differences were found between men with or without flank pain, except for stones, which were more frequently diagnosed on ultrasound if men complained of flank pains. Laboratory test results and radiological features had a poor predictive value. Men with suspected pyelonephritis did not evolve differently from those with suspected prostatitis. Monitoring and treatment of men with febrile urinary tract infection does not seem to depend on the existence of a suspected pyelonephritis suspected after the presence of a lumbar pain. The results of this study will be very useful for the development of future guidelines concerning the management of febrile UTI in men. Ambulatory management of febrile UTI is feasible and safe, requiring an efficient network for patient surveillance. 


\section{Acknowledgments}

SphereS Network 10 rue Ledion, Paris 75014, France, and Medical Emergency Department of Paris, 15 rue Jean Baptiste Berlier, Paris 75013, France.

\section{Author Contributions}

All the authors contributed in data management, analysis of the results and writing of the paper.

\section{Conflicts of Interest}

The authors declare no conflict of interest.

\section{References}

1. Nickel, J. Prostatitis and related conditions. In Campbell's Urology; Walsh, P., Retik, A.B., Darracott Vaughan, T.E., Jr., Wein, A.J., Eds.; SaundersWB: Philadelphia, PA, USA, 2002; pp. 60-630.

2. Auzanneau, C.; Manunta, A.; Vincendeau, S.; Patard, J.J.; Guillé, F.; Lobel, B. Management of acute prostatitis, based on a series of 100 cases. Prog. Urol. 2005, 15, 40-44.

3. Etienne, M.; Chavanet, P.; Sibert, L.; Michel, F.; Levesque, H.; Levesque, B.; Doucet, J.; Pfitzenmeyer, P.; Caron, F. Acute bacterial prostatitis: Heterogeneity in diagnostic criteria and management. Retrospective multicentric analysis of 371 patients diagnosed with acute prostatitis. BMC Infect. Dis. 2008, doi:10.1186/1471-2334-8-12.

4. Ozden, E.; Bostanci, Y.; Yakupoglu, K.Y.; Akdeniz, E.; Yilmaz, A.F.; Tulek, N.; Sarikaya, S. Incidence of acute prostatitis caused by extended-spectrum beta-lactamase-producing Escherichia coli after transrectal prostate biopsy. Urology 2009, 74, 119-223.

5. Shigehara, K.; Miyagi, T.; Nakashima, T.; Shimamura, M. Acute bacterial prostatitis after transrectal prostate needle biopsy: Clinical analysis. J. Infect. Chemother. 2008, 14, 40-43.

6. Stoica, G.; Cariou, G.; Colau, A.; Cortesse, A.; Hoffmann, P.; Schaetz, A.; Sellam, R. Epidemiology and treatment of acute prostatitis after prostatic biopsy. Prog. Urol. 2007, 17, 960-963.

7. Etienne, M.; Pestel-Caron, M.; Chapuzet, C.; Bourgeois, I.; Chavanet, P.; Caron1, F. Should blood cultures be performed for patients with acute prostatitis? J. Clin. Microbiol. 2010, 48, 1935-1938.

8. Koeijers, J.; Kessels, A.G.; Nys, S.; Bartelds, A.; Donker, G.; Stobberingh, E.E.; Verbon, A. Evaluation of the nitrite and leukocyte esterase activity tests for the diagnosis of acute symptomatic urinary tract infection in men. Clin. Infect. Dis. 2007, 45, 894-896. 


\title{
Chapter 8
}

\section{Clinical Efficacy of a Single Two Gram Dose of Azithromycin Extended Release for Male Patients with Urethritis}

\author{
Satoshi Takahashi, Hiroshi Kiyota, Shin Ito, Akihiko Iwasawa, Yoshiki Hiyama, \\ Teruhisa Uehara, Koji Ichihara, Jiro Hashimoto, Naoya Masumori, Kenichi Sunaoshi, \\ Koichi Takeda, Nobukazu Suzuki, Takahide Hosobe, Hirokazu Goto, Hidenori Suzuki \\ and Shoichi Onodera
}

\begin{abstract}
To clarify the clinical efficacy of a single oral $2 \mathrm{~g}$ dose of azithromycin extended-release for heterosexual male patients with urethritis, and the current antimicrobial sensitivity of Neisseria gonorrhoeae to azithromycin, a prospective clinical trial was conducted from 2011-2013. In patients with gonococcal urethritis, the eradication rate was $90.9 \%$ (30 of 33). The susceptibility rates of isolated Neisseria gonorrhoeae strains to ceftriaxone, spectinomycin, cefixime and azithromycin were 100\%, 100\%, 95.3\% (41/43) and 37.2\% (16/43), respectively. In the patients with nongonococcal urethritis, the eradication rate was $90.0 \%$ (45 of 50). The microbiological eradication rates for the pathogens were $90.9 \%(30 / 33)$ for Neisseria gonorrhoeae, 91.5\% (43/47) for Chlamydia trachomatis, $71.4 \%$ (5/7) for Mycoplasma genitalium, and 100\% (13/13) for Ureaplasma urealyticum. The main adverse event was diarrhea and its manifestation rate was 35.2\% (32 of 120). The symptom of diarrhea was mostly temporary and resolved spontaneously. The conclusion was that the treatment regimen with a single oral $2 \mathrm{~g}$ dose of azithromycin extended-release would be effective for patients with urethritis. However, the antimicrobial susceptibilities of Neisseria gonorrhoeae and Mycoplasma genitalium should be carefully monitored because of possible treatment failure.
\end{abstract}

Reprinted from Antibiotics. Cite as: Takahashi, S.; Kiyota, H.; Ito, S.; Iwasawa, A.; Hiyama, Y.; Uehara, T.; Ichihara, K.; Hashimoto, J.; Masumori, N.; Sunaoshi, K. Clinical Efficacy of a Single Two Gram Dose of Azithromycin Extended Release for Male Patients with Urethritis. Antibiotics 2014, 3, 109-120.

\section{Introduction}

The pathogen of gonococcal urethritis (GU) is Neisseria gonorrhoeae and the principal $\mathrm{p}$ athogens of nongonococcal urethritis (NGU) are Chlamydia trachomatis, Mycoplasma genitalium, Ureaplasma urealyticum [1]. Clinical guidelines clearly indicate different treatment regimens for male patients with GU and NGU. The guideline published by the Centers for Disease Control and Prevention (CDC), USA, recommends a treatment regimen with both a single $250 \mathrm{mg}$ dose of ceftriaxone intramuscularly and a single $1 \mathrm{~g}$ dose of azithromycin (AZM) orally for patients with GU [2], and a regimen with a single $1 \mathrm{~g}$ dose of AZM orally for patients with NGU [3]. Therefore, in the current clinical situation, AZM is a key drug worthy of note. However, several recent reports 
showed that strains of $N$. gonorrhoeae [4-6] and M. genitalium [7,8] highly resistant to AZM occurred worldwide. In addition, recent clinical trials revealed the decreased efficacy of a $1 \mathrm{~g}$ dose of AZM against NGU [9].

AZM is a macrolide antimicrobial agent and its ideal treatment regimen should achieve a higher area under the curve (AUC) above minimal inhibitory concentrations (MICs) value due to the current theory on pharmacokinetics (PK) and pharmacodynamics (PD) [10]. However, the conventional type of AZM, called immediate release (IR), has an increased risk of adverse events if patients are treated with a dose of AZM IR $2 \mathrm{~g}$ [11]. To overcome this situation, a single $2 \mathrm{~g}$ dose of AZM extended release (ER) has been developed [12]. AZM ER microsphere formulation can delay the release of AZM and bypass the upper gastrointestinal motilin receptors. In addition, this formulation can decrease the release rate of AZM from the matrix which leads to reduced concentration-dependent local irritation on upper gastrointestinal mucosa. In Japan, AZM ER is available to treat patients with both GU and NGU because it is officially approved, although AZM ER is not available for patients with urethritis in most countries outside of Japan. Therefore, there have been few studies about the clinical efficacy of AZM ER for male patients with urethritis. The aim of this study was to clarify the clinical efficacy of single $2 \mathrm{~g}$ dose of AZM ER for male patients with GU and NGU, as well as the current antimicrobial efficacy of AZM against $N$. gonorrhoeae.

\section{Patients and Methods}

\subsection{Study Design}

This prospective, multi-institutional, open label, single-arm clinical study was conducted from September 2011 to August 2013. It was designed essentially according to Japanese guidelines [13].

\subsection{Patients}

This clinical study included heterosexual male patients with both GU and NGU who were 20 years old or older. Diagnosis of GU was done based on both symptoms, urethral pain and pus discharge on external urethral meatus, suspecting urethritis, suspecting urethritis [14] and positive $N$. gonorrhoeae detected by microscopic examination of Gram staining, culture of urethral pus discharge or a nucleic acid amplification test (NAAT) of first-voided urine (FVU). Diagnosis of NGU was done based on both symptoms suspected to be urethritis and negative $N$. gonorrhoeae examined by microscopic examination of culture of urethral pus discharge or commercially available NAAT of FVU. In addition, C. trachomatis, M. genitalium and U. urealyticum were also detected by a microbiological test using NAAT. The patients diagnosed with GU or NGU were treated with a single $2 \mathrm{~g}$ dose of AZM ER.

\subsection{Procedures for Detection of Pathogens}

At each clinic, pus discharge from the external urethral meatus was collected as the specimen for Gram staining and/or culture of $N$. gonorrhoeae. The culture method was described in detail in a previous report [15]. In brief, the specimen was applied on Thayer-Martin Selective Agar (Becton Dickinson, Cockeysville, MD, USA) and immediately transported in a Bio-Bag Environmental 
Chamber Type C (Becton, Dickinson) to Mitsubishi Chemical Medience Corporation, Tokyo. Then, it was incubated at $35{ }^{\circ} \mathrm{C}$ for $48 \mathrm{~h}$ in a $5 \% \mathrm{CO}_{2}$ atmosphere.

About $20 \mathrm{~mL}$ of FVU was taken from each patient and used as a specimen. C. trachomatis and N. gonorrhoeae were detected with the commercially available NAAT test kit by transcription-mediated amplification, polymerase chain reaction (PCR) or strand displacement amplification. M. genitalium and $U$. urealyticum were detected using a previously reported PCR method at Mitsubishi Chemical Medience Corporation [16].

\subsection{Antimicrobial Susceptibility Testing}

In this study, the minimum inhibitory concentrations (MICs) of eight antimicrobial agents were determined: penicillin G (PCG) (Wako Pure Chemical Industries, Osaka, Japan), ceftriaxone (CTRX) (Wako Pure Chemical Industries), cefodizime (CDZM) (Taiho Pharmaceutical Co., Tokyo, Japan), cefixime (CFIX) (Wako Pure Chemical Industries), spectinomycin (SPCM) (Wako Pure Chemical Industries), azithromycin (AZM) (LKT Laboratories, St. Paul, MN, USA), ciprofloxacin (CPFX) (Tokyo Chemical Industry Co., Tokyo, Japan) and levofloxacin (LVFX) (Tokyo Chemical Industry Co.). The MICs were determined by an agar dilution method according to a report (M07-A9 and M100-S23 Package) from the Clinical and Laboratory Standards Institute (CLSI). After incubation at $35{ }^{\circ} \mathrm{C}$ for $20 \mathrm{~h}$ in a $5 \% \mathrm{CO}_{2}$ atmosphere, the lowest concentration of the antimicrobial agent that could inhibit bacterial growth completely was defined as the MIC. In addition, the lowest concentration of the drug that could inhibit approximately $90 \%$ and $50 \%$ of strains was defined as the $\mathrm{MIC}_{90}$ and $\mathrm{MIC}_{50}$, respectively. According to the criteria of the CLSI (M100-S23), the MIC breakpoints for susceptibility and resistance were $\leqq 0.06 \mu \mathrm{g} / \mathrm{mL}$ and $\geqq 2 \mu \mathrm{g} / \mathrm{mL}$ for penicillin, respectively. For CTRX and CFIX the breakpoint for susceptibility was $\leqq 0.25 \mu \mathrm{g} / \mathrm{mL}$. For SPCM and CPFX the susceptibility and resistance breakpoints were $\leqq 32 \mu \mathrm{g} / \mathrm{mL}$ and $\geqq 128 \mu \mathrm{g} / \mathrm{mL}$, and $\leqq 0.06 \mu \mathrm{g} / \mathrm{mL}$ and $\geqq 1 \mu \mathrm{g} / \mathrm{mL}$, respectively. The MIC breakpoint of AZM was not defined; however, for it we used the MIC breakpoint for susceptibility and resistance of $\leqq 0.25 \mu \mathrm{g} / \mathrm{mL}$ and $\geqq 1 \mu \mathrm{g} / \mathrm{mL}$, respectively, according to the European Committee on Antimicrobial Susceptibility Testing (EUCAST [17]). The MIC breakpoints of CDZM and LVFX were not defined either; therefore, we used the MIC breakpoint for susceptibility and resistance of CDZM of $\leqq 0.06 \mu \mathrm{g} / \mathrm{mL}$ and $\geqq 0.5 \mu \mathrm{g} / \mathrm{mL}$, and those of LVFX were $\leqq 0.12 \mu \mathrm{g} / \mathrm{mL}$ and $\geqq 2 \mu \mathrm{g} / \mathrm{mL}$, respectively.

\subsection{Outcome}

We evaluated both microbiological and clinical cure outcomes judged 1-4 weeks after the completion of AZM ER treatment. The definition of each microbiological and clinical cure was essentially using the Japanese guideline for clinical research. In the Japanese guideline [13], the optimal time to evaluate bacteriological and clinical cure outcomes is $2-4$ weeks post-treatment; however, we modified the duration for judgment because AZM ER was given as a single dose.

In the patients with $\mathrm{GU}$, the microbiological outcome was the primary endpoint or assessment, and eradication meant no $N$. gonorrhoeae detected by culture or by NAAT posttreatment. In those with GU, failure of microbiological outcome meant $N$. gonorrhoeae detected by culture or modification 
of treatment, including a change or addition of antimicrobial agents. In those patients, clinical cure meant no symptoms derived from GU.

In the patients with NGU, the microbiological outcome was the primary endpoint or assessment, and eradication meant no $C$. trachomatis, M. genitalium, or U. urealyticum detected by NAAT posttreatment. In those with $\mathrm{NGU}$, failure as microbiological outcome meant $C$. trachomatis, M. genitalium or U. urealyticum detected by culture or modification of treatment including a change or addition of antimicrobial agents. In those patients, clinical cure meant no symptoms derived from NGU and failure meant continuation of a symptom derived from urethritis or with changes/additions of antimicrobial agents post-treatment. Only the clinical outcome was analyzed in the patients without any microbial detection.

\subsection{Assessment of Adverse Events}

At the second visit, the doctors interviewed each patient in detail about whether adverse events, including diarrhea, abdominal pain, nausea and so on, had occurred. The grade of adverse events was determined according to Common Terminology Criteria for Adverse Events (CTCAE) v4.0.

\subsection{Ethical Considerations}

This clinical study design was approved by Institutional Review Board of Sapporo Medical University Hospital ([18]; Nos. 22-128, 24-3011) and written informed consent was obtained from each subject. This clinical study is registered in the University Hospital Medical Information Network Clinical Trial Registry (UMIN-CTR [19]; UMIN ID; UMIN000006359).

\section{Results}

A total of 200 patients were included in this study (Figure 1). Of these 200 patients, 55 were diagnosed with GU, including concomitant, 10 positive for C. trachomatis, one with M. genitalium and two with $U$. urealyticum. There were $43 \mathrm{~N}$. gonorrhoeae strains isolated from the patients with GU and the MICs against these strains were measured. The microbiological and clinical efficacy were analyzed in 33 patients with GU including for analysis and eight patients of concomitant urethritis with C. trachomatis, one of urethritis with M. genitalium and one with U. urealyticum. In these 33 patients with GU, median age was 33 (range: 21-50) years old. The median duration from the completion of treatment to the second visit was 9 (range: 7-27) days. Twenty-two patients with GU were excluded from this study, because of no second visit for 16, lack of data for three and violation of the evaluation period in three. There were 145 patients who were diagnosed as having NGU. Of these 145 patients, 58 were excluded from this study, because of no second visit for 45 and violation of the evaluation period for 13. Finally, 87 could be analyzed, and they were classified into 35 cases of urethritis with $C$. trachomatis, three with $M$. genitalium, five with $U$. urealyticum, four with C. trachomatis and U. urealyticum, three with M. genitalium and U. urealyticum, and 37 without any microbial detection. In these 87 patients with NGU, median age was 33 (range: 20-65) years old. The median duration from the completion of treatment to the second visit was 9 (range: 7-27) days. Of those patients included in the analysis, none had sexual intercourse between the first and second visits and, based on the interviews, they took the drug as prescribed. 
The MIC of each antimicrobial agent was measured for $43 \mathrm{~N}$. gonorrhoeae strains (Table 1). The rate of susceptibility to PCG was 9.3\% (4/43) and that for CPFX or LVFX was $20.9 \%$ (9/43). The rates for CTRX, SPCM and CFIX were 100\%, 100\% and 95.3\% (41/43), respectively. That for AZM was 37.2\% (16/43).

In the patients with $\mathrm{GU}$, the eradication rate, as the microbiological outcome, was $90.9 \%$ (30 of 33 ) and the cure rate, as the clinical outcome, was $84.8 \%$ (28 of 33). Of the three patients with GU that could not be eradicated by a single $2 \mathrm{~g}$ dose of AZM ER, one was treated by a single $1 \mathrm{~g}$ dose of intravenous CTRX and his pathogen was eradicated. The other two patients could not be followed-up further. The symptom of pus discharge had not disappeared in any of the three patients at the second visit. The MICs of AZM ER in these three patients with microbiological failure were $0.5 \mu \mathrm{g} / \mathrm{mL}, 1 \mu \mathrm{g} / \mathrm{mL}$, and $2 \mu \mathrm{g} / \mathrm{mL}$ (Table 2). If an MIC with complete eradication is defined as the MIC breakpoint of susceptibility, $0.25 \mu \mathrm{g} / \mathrm{mL}$ was the MIC breakpoint for AZM in this study. In one patient with GU, $N$. gonorrhoeae was eradicated; however, M. genitalium was not eradicated. In the patients with NGU, the microbiological eradication rate was $90.0 \%$ (45 of 50) and the clinical cure rate was 94.3\% (82 of 87) (Table 3). Four patients with treatment failure of C. trachomatis-positive urethritis did not complain of symptoms related to urethritis at the second visit. Three patients did not visit the clinic again and no further follow-up could be done. Only one patient visited again 60 days after the first visit and was treated with minocycline successfully.

Figure 1. Flow chart for assessment of patients: gonococcal urethritis = GU, nongonococcal urethritis $=\mathrm{NGU})$.

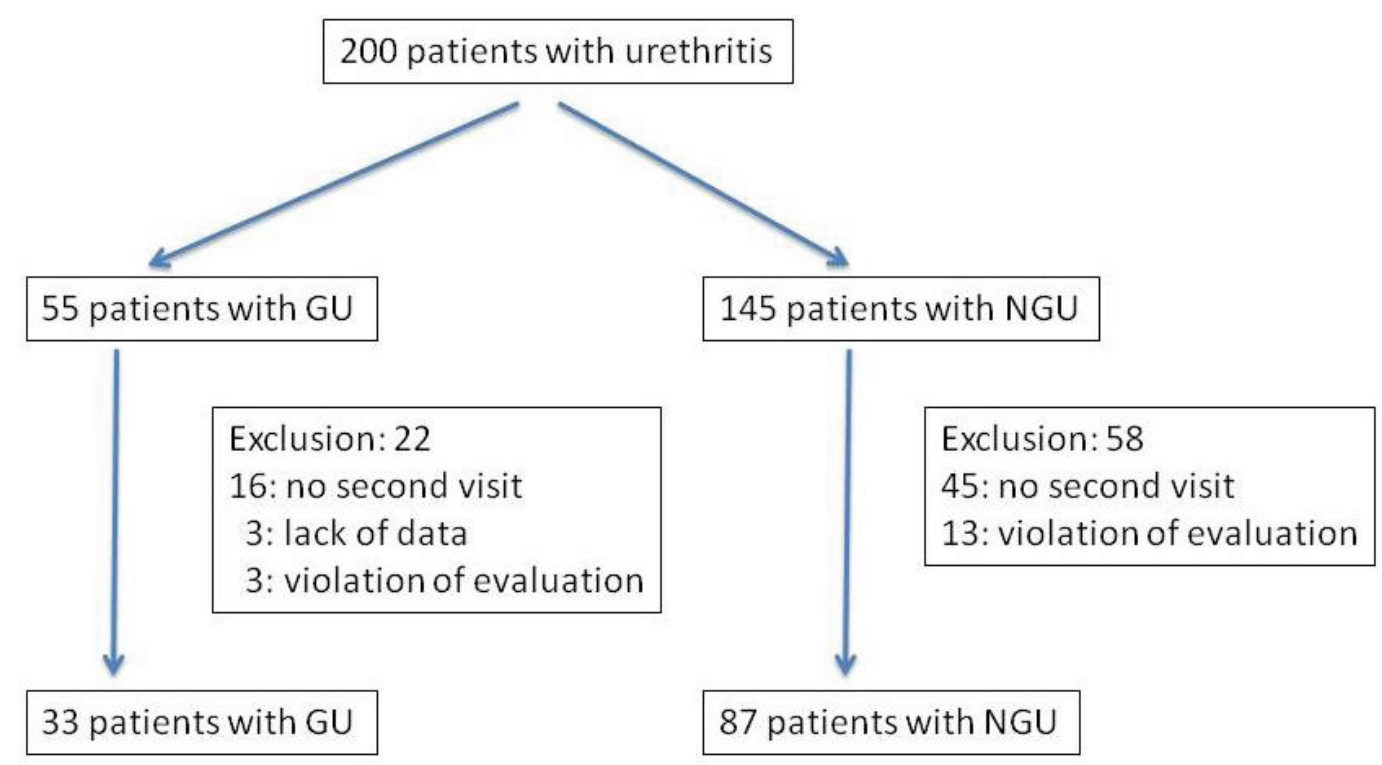

The microbiological eradication rates of the pathogens were $90.9 \%(30 / 33)$ for $N$. gonorrhoeae, 91.5\% (43/47) for C. trachomatis, 71.4\% (5/7) for M. genitalium, and 100\% (13/13) for U. urealyticum.

The interview at the second visit showed that diarrhea was the main adverse event and its manifestation rate was $35.2 \%$ (32 of 120 ). However, this diarrhea was mostly temporary and no further additional treatment was necessary for almost all the patients with diarrhea. In the patients having temporary diarrhea, the symptom was resolved within one day when the patient took AZM ER. Therefore, the degree of this adverse event was less than grade 1 defined by the CTCAE. 
Table 1. The MIC distribution of each antimicrobial agent against 43 clinically isolated N. gonorrhoeae strains.

\begin{tabular}{|c|c|c|c|c|c|c|c|c|c|c|c|c|c|c|c|c|c|}
\hline \multirow{2}{*}{$\begin{array}{c}\text { Antimicrobial } \\
\text { Agents }\end{array}$} & \multicolumn{17}{|c|}{$\operatorname{MICs}(\mu \mathrm{g} / \mathrm{mL})$} \\
\hline & $\leqq 0.001$ & 0.002 & 0.004 & 0.008 & 0.015 & 0.03 & 0.06 & 0.12 & 0.25 & 0.5 & 1 & 2 & 4 & 8 & 16 & 32 & 64 \\
\hline PCG & - & - & - & - & 2 & - & 2 & 9 & 7 & 9 & 6 & 6 & 1 & - & - & 1 & - \\
\hline CTRX & 2 & 4 & 6 & 6 & 5 & 11 & 7 & 2 & - & - & - & - & - & - & - & - & - \\
\hline CDZM & - & 1 & 3 & 8 & 2 & 9 & 11 & 8 & 1 & - & - & - & - & - & - & - & - \\
\hline CFIX & - & 1 & 1 & 6 & 5 & 2 & 9 & 14 & 3 & 2 & - & - & - & - & - & - & - \\
\hline SPCM & - & - & - & - & - & - & - & - & - & - & - & - & - & 8 & 22 & 13 & - \\
\hline AZM & - & - & - & - & 1 & - & 6 & 5 & 4 & 9 & 11 & 5 & 1 & - & 1 & - & - \\
\hline CPFX & - & - & 3 & 4 & 2 & - & - & - & - & - & - & 1 & - & 4 & 8 & 17 & 4 \\
\hline LVFX & - & - & 3 & 4 & 2 & - & - & - & - & - & 1 & 1 & 4 & 18 & 9 & 1 & - \\
\hline
\end{tabular}

PCG: penicillin G; CTRX: ceftriaxone; CDZM: cefodizime; CFIX: cefixime; SPCM: spectinomycin; AZM: azithromycin; CPFX: ciprofloxacin; LVFX: levofloxacin.

\section{Discussion}

There have been several critical issues concerning how to treat patients with GU or NGU properly. Among these, the occurrence and development of drug-resistant $N$. gonorrhoeae strains has been the focus of worldwide attention [20]. The updated treatment regimen recommended by the CDC of the USA for patients with GU is CTRX $250 \mathrm{mg}$ in a single muscular dose plus AZM $1 \mathrm{~g}$ orally in a single dose or doxycycline $100 \mathrm{mg}$ orally twice daily for seven days [2]. In Japan, the guidelines of the Japanese Society for Sexually Transmitted Infections (STI), the Japanese Association for Infectious Diseases and Japanese Society of Chemotherapy commonly recommend a treatment regimen using CTRX $1 \mathrm{~g}$ in a single intravenous administration, CDZM $1 \mathrm{~g}$ in a single intravenous administration or SPCM $2 \mathrm{~g}$ in a single intramuscular dose. CTRX has been a key drug for the treatment of GU; however, the Gonococcal Isolate Surveillance Project (GISP) (2000-2010) in the USA showed that the frequencies of $N$. gonorrhoeae isolates with CFIX MICs $\geqq 0.25 \mu \mathrm{g} / \mathrm{mL}$ and CTRX MICs $\geqq 0.125 \mu \mathrm{g} / \mathrm{mL}$ have been increasing gradually [21]. Although the prevalence of $N$. gonorrhoeae isolates with elevated MICs remains low, the susceptibility rates to CFIX and CTRX are decreasing. In addition, a CTRX-resistant N. gonorrhoeae strain was isolated in 2009 in Kyoto, Japan [22]. Thus, in the current situation there will inevitably be a need for alternative effective treatment regimens for GU.

AZM is a macrolide antimicrobial agent that has efficacy against pathogens of urethritis [23]. In general, $1 \mathrm{~g}$ of AZM immediate release (IR) is widely used for the treatment of urethritis according to several guidelines [3]. Although the treatment efficacy with AZM IR $1 \mathrm{~g}$ orally is good [23], according to the principles of PK/PD, a greater AUC/MIC could probably obtain better treatment efficacy by a macrolide [10]. However, a relatively high frequency of gastrointestinal adverse events could occur in patients treated with AZM IR 2 g orally in a single dose [11], although the treatment efficacy of AZM IR $2 \mathrm{~g}$ is as good as that of CTRX $250 \mathrm{mg}$ intramuscular administration in patients with GU [11]. Therefore, efforts have been made to achieve a greater AUC/MIC with AZM ER and to reduce adverse events [12]. According to a report from India, 
the AZM IR 2 g single dose regimen achieved 100\% microbiological efficacy for patients with GU [24].

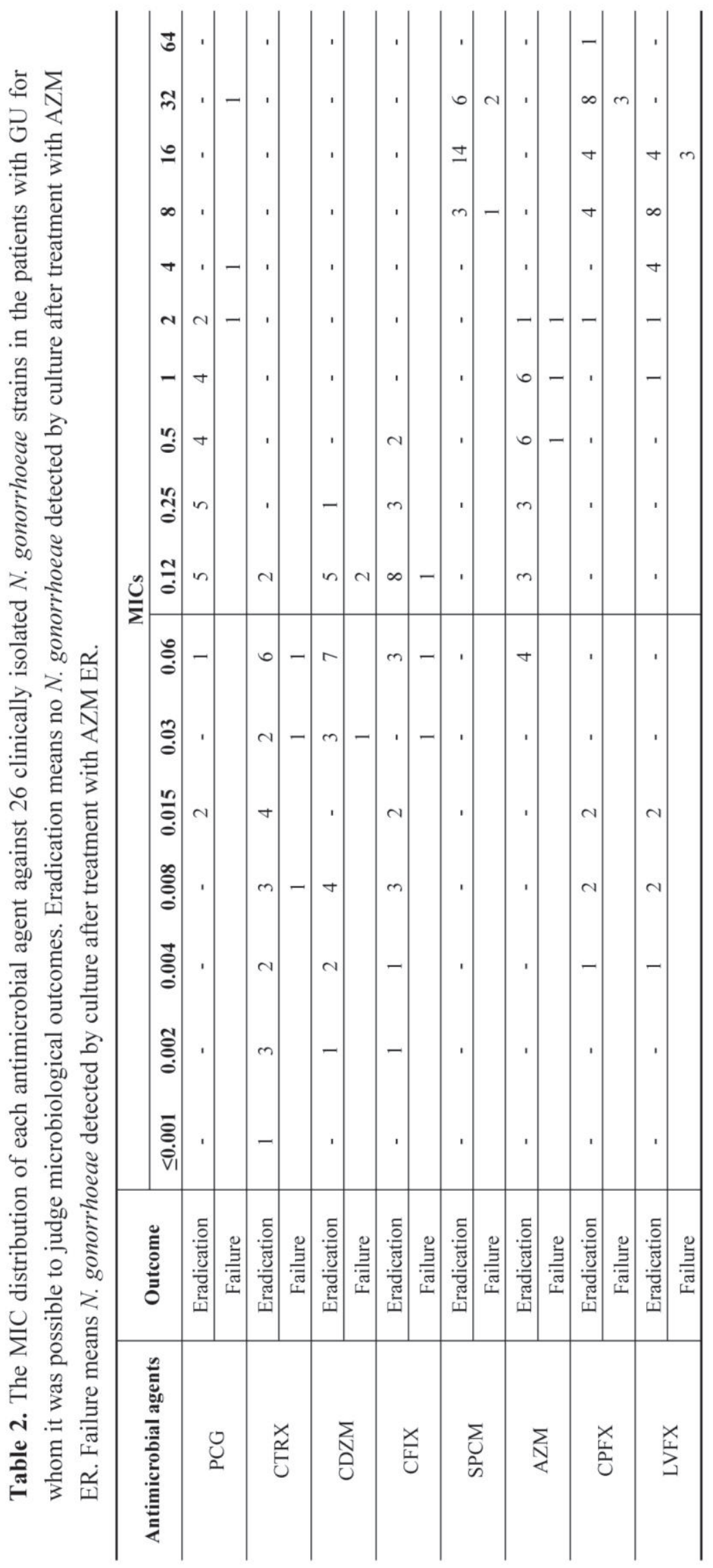


Table 3. Microbiological and clinical outcomes in patients with gonococcal urethritis (GU) and nongonococcal urethritis (NGU).

\begin{tabular}{|c|c|c|c|c|c|}
\hline Urethritis & Pathogen Coinfection & Number & $\begin{array}{c}\text { Eradication } \\
\text { (Number) }\end{array}$ & $\begin{array}{c}\text { Cure } \\
\text { (Number) }\end{array}$ & $\begin{array}{c}\text { Pathogen of Treatment } \\
\text { Failure and Number }\end{array}$ \\
\hline \multirow{4}{*}{ GU } & None & 23 & \multirow{4}{*}{30} & \multirow{4}{*}{28} & \multirow{4}{*}{ M. genitalium in 1} \\
\hline & C. trachomatis & 8 & & & \\
\hline & M. genitalium & 1 & & & \\
\hline & U. urealyticum & 1 & & & \\
\hline Urethritis & Pathogen infection & Number & Eradication & Cure & $\begin{array}{c}\text { Pathogen in failure } \\
\text { and number }\end{array}$ \\
\hline \multirow{6}{*}{ NGU } & C. trachomatis & 35 & 31 & 34 & \multirow{6}{*}{ C. trachomatis in 4} \\
\hline & M. genitalium & 3 & 2 & 3 & \\
\hline & U. urealyticum & 5 & 5 & 5 & \\
\hline & C. trachomatis and $U$. urealyticum & 4 & 4 & 4 & \\
\hline & M. genitalium and U. urealyticum & 3 & 3 & 3 & \\
\hline & Without any microbial detection & 37 & - & 33 & \\
\hline
\end{tabular}

In this study, the microbiological eradication rate was $90.9 \%$ in the patients with GU. This eradication rate was relatively low compared to those in previous reports [21]. The MIC distribution of AZM also shifted to higher MICs and the rate of susceptibility to AZM was $37.2 \%$ if the MIC breakpoint of susceptibility was defined as $0.25 \mu \mathrm{g} / \mathrm{mL}$. This report from Japan suggests that the susceptibility to AZM might be decreasing, though two recent reports showed that the susceptibility rate was high, with no resistant strains identified $[25,26]$. In our study, three patients with GU had treatment failure, with $N$. gonorrhoeae strains having MICs of $0.5 \mu \mathrm{g} / \mathrm{mL}, 1 \mathrm{ug} / \mathrm{mL}$ and $2 \mu \mathrm{g} / \mathrm{mL}$. In these MIC groups, six patients with an MIC of $0.5 \mu \mathrm{g} / \mathrm{mL}$, six with $1 \mathrm{ug} / \mathrm{mL}$ and one with $2 \mu \mathrm{g} / \mathrm{mL}$ were treated successfully and the $N$. gonorrhoeae strains were completely eradicated. This study suggested that the AZM ER $2 \mathrm{~g}$ single dose treatment regimen is promising and adequate to eradicate $N$. gonorrhoeae. This result suggested that future guidelines should limit the treatment to AZM IR $1 \mathrm{~g}$. However, recent reports from the USA and Europe revealed that the susceptibility of $N$. gonorrhoeae to AZM has been decreasing and highly resistant isolates were identified in the USA [4-6,27]. Therefore, a nationwide surveillance system such as the GISP or the surveillance system of the Japanese Association for Infectious Diseases, Japanese Society of Chemotherapy and the Japanese Society for Clinical Microbiology, is indispensable for the management of GU. In addition, it is important to check the MIC breakpoint of susceptibility because a higher AUC/MIC due to the AZM ER $2 \mathrm{~g}$ single dose may contribute to a better outcome than treatment with AZM IR $1 \mathrm{~g}$.

The treatment regimen using a single $1 \mathrm{~g}$ oral dose of AZM IR is a suitable recommendation for patients with NGU [21]. In this study, the treatment efficacy of the single 2 g AZM ER oral dose was excellent and the results were similar to those of our previous reports [23,28-30]. Therefore, this treatment regimen can also be strongly recommended for patients with NGU because of its high treatment efficacy as well as being an ideal dose based on PK/PD principles. However, there has been an issue about treatment failure against $M$. genitalium recently $[8,9]$. In our study, the microbiological eradication rate was $71.5 \%$ (5/7) for $M$. genitalium, although the sample number was small. A recent 
report from the USA showed that treatment failure was common among patients with M. genitaliumpositive NGU who received a single $1 \mathrm{~g}$ dose of AZM IR [9]. In addition, highly AZM-resistant M. genitalium has been isolated from STI patients [27]. Because the sample number was limited in this study, the definitive treatment efficacy of AZM ER $2 \mathrm{~g}$ for patients with M. genitalium-positive NGU remains to be determined. However, this treatment regimen may be promising for patients with C. trachomatis-positive and U. urealyticum-positive NGU. If the AZM ER 2 g single dose regimen fails to cure patients with M. genitalium-positive NGU, sitafloxacin (STFX), one of the newer oral fluoroquinolones has strong clinical activity against M. genitalium [29,30]. Therefore, the STFX $100 \mathrm{mg}$ twice daily seven days regimen can be an effective alternative to the AZM ER $2 \mathrm{~g}$ regimen for patients with M. genitalium-positive NGU.

Although diarrhea was the main side effect, with a manifestation rate of $35.2 \%$, it occurred temporarily and no patients with diarrhea needed additional treatment. A previous report clarified that gastrointestinal side effects, mainly nausea and vomiting, occurred in $35.3 \%$ of patients who received AZM IR $2 \mathrm{~g}$, with a moderate degree in 10.1\% and a severe one in 2.9\% [11]. Although the frequency of side effects in the patients who received AZM ER $2 \mathrm{~g}$ was not very low, they were acceptable enough because of their temporary nature and limited degree.

This clinical study has several limitations. The relatively small sample size might not have adequate statistical power. Some patients with treatment failure were lost to follow-up and we could not obtain the results of second-line treatment. Unfortunately, the sample number for M. genitalium was relatively small and it was hard to determine the exact treatment efficacy of the AZM ER $2 \mathrm{~g}$ single dose against this pathogen. However, this is the first clinical study to evaluate the clinical efficacy of AZM ER $2 \mathrm{~g}$ oral single dose treatment for patients with GU and NGU. This study demonstrated the excellent treatment efficacy and adequate tolerability of the oral $2 \mathrm{~g}$ single dose AZM ER regimen. A future randomized controlled study with a larger series will be able to further clarify the treatment efficacy for patients with GU and NGU.

\section{Conclusions}

The treatment regimen with a single $2 \mathrm{~g}$ oral dose of AZM ER is effective for patients with GU and NGU. Although the frequency of side effects was $35.2 \%$, these side effects were acceptable because they were mild and temporary, and this treatment regimen could be used safely. However, the antimicrobial susceptibilities of $N$. gonorrhoeae and M. genitalium and clinical efficacy in patients with GU and NGU should be carefully monitored because of possible treatment failure of AZM in the future.

\section{Author Contributions}

Satoshi Takahashi initiated the study, collected data, participated in data analysis and wrote the first draft of the manuscript. Hiroshi Kiyota, Shin Ito, Naoya Masumori, and Shoichi Onodera initiated the study, participated in data analysis and edited the manuscript. Akihiko Iwasawa, Yoshiki Hiyama, Teruhisa Uehara, Koji Ichihara, Jiro Hashimoto, Kenichi Sunaoshi, Koichi Takeda, Nobukazu Suzuki, Takahide Hosobe, Hirokazu Goto, and Hidenori Suzuki collected data and edited the manuscript. 


\section{Conflicts of Interest}

The authors declare no conflict of interest.

\section{References}

1. Wetmore, C.M.; Manhart, L.E.; Lowens, M.S.; Golden, M.R.; Whittington, W.L.; Xet-Mull, A.M.; Astete, S.G.; McFarland, N.L.; McDougal, S.J.; Totten, P.A. Demographic, behavioral, and clinical characteristics of men with nongonococcal urethritis differ by etiology: A case-comparison study. Sex. Transm. Dis. 2011, 38, 180-186.

2. Centers for Disease Control and Prevention. Update to CDC's sexually transmitted diseases treatment guidelines, 2010: Oral cephalosporins no longer a recommended treatment for gonococcal infections. Morb. Mortal. Wkly. Rep. 2012, 10, 590-594.

3. Workowski, K.A.; Berman, S.; Centers for Disease Control and Prevention. Sexually transmitted diseases treatment guidelines, 2010. Morb. Mortal. Wkly. Rep. 2010, 59, 1-110.

4. Chisholm, S.A.; Neal, T.J.; Alawattegama, A.B.; Birley, H.D.; Howe, R.A.; Ison, C.A. Emergence of high-level azithromycin resistance in Neisseria gonorrhoeae in England and Wales. J. Antimicrob. Chemother. 2009, 64, 353-358.

5. Galarza, P.G.; Alcala, B.; Salcedo, C.; Canigia, L.F.; Buscemi, L.; Pagano, I.; Oviedo, C.; Vázquez, J.A. Emergence of high level azithromycin-resistant Neisseria gonorrhoeae strain isolated in Argentina. Sex. Transm. Dis. 2009, 36, 787-788.

6. Starnino, S.; Stefanelli, P. Neisseria gonorrhoeae Italian Study G. Azithromycin-resistant Neisseria gonorrhoeae strains recently isolated in Italy. J. Antimicrob. Chemother. 2009, 63, $1200-1204$.

7. Bradshaw, C.S.; Jensen, J.S.; Tabrizi, S.N.; Read, T.R.; Garland, S.M.; Hopkins, C.A.; Moss, L.M.; Fairley, C.K. Azithromycin failure in Mycoplasma genitalium urethritis. Emerg. Infect. Dis. 2006, 12, 1149-1152.

8. Jensen, J.S.; Bradshaw, C.S.; Tabrizi, S.N.; Fairley, C.K.; Hamasuna, R. Azithromycin treatment failure in Mycoplasma genitalium-positive patients with nongonococcal urethritis is associated with induced macrolide resistance. Clin. Infect. Dis. 2008, 47, 1546-1553.

9. Manhart, L.E.; Gillespie, C.W.; Lowens, M.S.; Khosropour, C.M.; Colombara, D.V.; Golden, M.R.; Hakhu, N.R.; Thomas, K.K.; Hughes, J.P.; Jensen, N.L.; et al. Standard treatment regimens for nongonococcal urethritis have similar but declining cure rates: A randomized controlled trial. Clin. Infect. Dis. 2013, 56, 934-942.

10. Jain, R.; Danziger, L.H. The macrolide antibiotics: A pharmacokinetic and pharmacodynamic overview. Curr. Pharm. Des. 2004, 10, 3045-3053.

11. Handsfield, H.H.; Dalu, Z.A.; Martin, D.H.; Douglas, J.M., Jr.; McCarty, J.M.; Schlossberg, D.; Azithromycin Gonorrhea Study Group. Multicenter trial of single-dose azithromycin vs. ceftriaxone in the treatment of uncomplicated gonorrhea. Sex Transm. Dis. 1994, 21, 107-111.

12. Chandra, R.; Liu, P.; Breen, J.D.; Fisher, J.; Xie, C.; LaBadie, R.; Benner, R.J.; Benincosa, L.J.; Sharma, A. Clinical pharmacokinetics and gastrointestinal tolerability of a novel extended-release microsphere formulation of azithromycin. Clin. Pharm. 2007, 46, 247-259. 
13. Yasuda, M.; Takahashi, S.; Kiyota, H.; Ishikawa, K.; Takahashi, A.; Yamamoto, S.; Arakawa, S.; Monden, K.; Muratani, T.; Hamasuna, R.; et al. Japanese guideline for clinical research of antimicrobial agents on urogenital infections: The first edition. J. Infect. Chemother. 2011, 17, 579-594.

14. Takahashi, S.; Takeyama, K.; Kunishima, Y.; Takeda, K.; Suzuki, N.; Nishimura, M.; Furuya, R.; Tsukamoto, T. Analysis of clinical manifestations of male patients with urethritis. J. Infect. Chemother. 2006, 12, 283-286.

15. Saika, T.; Nishiyama, T.; Kanayama, A.; Kobayashi, I.; Nakayama, H.; Tanaka, M.; Naito, S. Comparison of Neisseria gonorrhoeae isolates from the genital tract and pharynx of two gonorrhea patients. J. Infect. Chemother. 2001, 7, 175-179.

16. Yoshida, T.; Maeda, S.; Deguchi, T.; Ishiko, H. Phylogeny-based rapid identification of mycoplasmas and ureaplasmas from urethritis patients. J. Clin. Microbiol. 2002, 40, 105-110.

17. The European Committee on Antimicrobial Susceptibility Testing-EUCAST. Available online: http://www.escmid.org/reseach_projects/eucast/ (accessed on 19 March 2014).

18. Institutional Review Board of Sapporo Medical University Hospital. Available online: http://web.sapmed.ac.jp/byoin/chiken/irb.html/ (accessed on 19 March 2014).

19. University Hospital Medical Information Network Clinical Trial Registry (UMIN-CTR). Available online: http://www.umin.ac.jp/ctr/index-j.htm/ (accessed on 19 March 2014).

20. Bolan, G.A.; Sparling, P.F.; Wasserheit, J.N. The emerging threat of untreatable gonococcal infection. N. Engl. J. Med. 2012, 366, 485-487.

21. Centers for Disease Control and Prevention. Cephalosporin susceptibility among Neisseria gonorrhoeae isolates-United States, 2000-2010. Morb. Mortal. Wkly. Rep. 2011, 60, 873-877.

22. Ohnishi, M.; Saika, T.; Hoshina, S.; Iwasaku, K.; Nakayama, S.; Watanabe, H.; Kitawaki, J. Ceftriaxone-resistant Neisseria gonorrhoeae, Japan. Emerg. Infect. Dis. 2011, 17, 148-149.

23. Takahashi, S.; Matsukawa, M.; Kurimura, Y.; Takeyama, K.; Kunishima, Y.; Iwasawa, A.; Koroku, M.; Tanda, H.; Suzuki, N.; Takagi, Y.; et al. Clinical efficacy of azithromycin for male nongonococcal urethritis. J. Infect. Chemother. 2008, 14, 409-412.

24. Khaki, P.; Bhalla, P.; Sharma, A.; Kumar, V. Correlation between in vitro susceptibility and treatment outcome with azithromycin in gonorrhoea: A prospective study. Ind. J. Med. Microbiol. 2007, 25, 354-357.

25. Endo, K.; Onodera, S.; Kiyota, H.; Suzuki, H.; Hosobe, T.; Naruoka, T.; Sanuki, K. Drug-susceptibilities of Neisseria gonorrhoeae strains isolated from male patients with gonococcal urethritis against antimicrobial agents-Comparisons from 2006 to 2010. Jpn. J. Chemother. 2011, 59, 308-312.

26. Hamasuna, R.; Yasuda, M.; Ishikawa, K.; Uehara, S.; Takahashi, S.; Hayami, H.; Yamamoto, S.; Matsumoto, T.; Minamitani, S.; Watanabe, A.; et al. Nationwide surveillance of the antimicrobial susceptibility of Neisseria gonorrhoeae from male urethritis in Japan. J. Infect. Chemother. 2013, 19, 571-578.

27. Katz, A.R.; Komeya, A.Y.; Soge, O.O.; Kiaha, M.I.; Lee, M.V.; Wasserman, G.M.; Maningas, E.V.; Whelen, A.C.; Kirkcaldy, R.D.; Shapiro, S.J.; et al. Neisseria gonorrhoeae with high-level resistance to azithromycin: Case report of the first isolate identified in the United States. Clin. Infect. Dis. 2012, 54, 841-843. 
28. Takahashi, S.; Ichihara, K.; Hashimoto, J.; Kurimura, Y.; Iwasawa, A.; Hayashi, K.; Sunaoshi, K.; Takeda, K.; Suzuki, N.; Satoh, T.; et al. Clinical efficacy of levofloxacin $500 \mathrm{mg}$ once daily for 7 days for patients with non-gonococcal urethritis. J. Infect. Chemother. 2011, 17, 392-396.

29. Ito, S.; Yasuda, M.; Seike, K.; Sugawara, T.; Tsuchiya, T.; Yokoi, S.; Nakano, M.; Deguchi, T. Clinical and microbiological outcomes in treatment of men with non-gonococcal urethritis with a 100-mg twice-daily dose regimen of sitafloxacin. J. Infect. Chemother. 2012, 18, 414-418.

30. Takahashi, S.; Hamasuna, R.; Yasuda, M.; Ito, S.; Ito, K.; Kawai, S.; Yamaguchi, T.; Satoh, T.; Sunaoshi, K.; Takeda, K.; et al. Clinical efficacy of sitafloxacin $100 \mathrm{mg}$ twice daily for 7 days for patients with non-gonococcal urethritis. J. Infect. Chemother. 2013, 19, 941-945. 


\title{
Chapter 9
}

\section{Ureteral Stents and Foley Catheters-Associated Urinary Tract Infections: The Role of Coatings and Materials in Infection Prevention}

\author{
Joey Lo, Dirk Lange and Ben H. Chew
}

\begin{abstract}
Urinary tract infections affect many patients, especially those who are admitted to hospital and receive a bladder catheter for drainage. Catheter associated urinary tract infections are some of the most common hospital infections and cost the health care system billions of dollars. Early removal is one of the mainstays of prevention as $100 \%$ of catheters become colonized. Patients with ureteral stents are also affected by infection and antibiotic therapy alone may not be the answer. We will review the current evidence on how to prevent infections of urinary biomaterials by using different coatings, new materials, and drug eluting technologies to decrease infection rates of ureteral stents and catheters.
\end{abstract}

Reprinted from Antibiotics. Cite as: Lo, J.; Lange, D.; Chew, B.H. Ureteral Stents and Foley Catheters-Associated Urinary Tract Infections: The Role of Coatings and Materials in Infection Prevention. Antibiotics 2014, 3, 87-97.

\section{Introduction}

Catheter-associated urinary tract infections (CAUTI) are the most common source of hospital-acquired infections [1]. They account for $80 \%$ of all nosocomial infections worldwide, with approximately 450,000 cases in the United States alone annually [1]. As of 2008, treatment of these hospital-acquired infections is no longer eligible for reimbursement from the US Centres for Medicare and Medicaid Services, as they are considered to be preventable [2]. With direct treatment of CAUTIs amounting to over $\$ 350$ million per year, it is crucial to prevent such infection [1]. Ureteral stents are hollow tubes used to facilitate the drainage of urine from the kidney to the bladder and are often used after treatment for kidney stones; since these are foreign bodies within the urinary tract system, they can also often lead to urinary tract infection. Current strategies to reduce ureteral stent infection have been mostly unsuccessful and it remains a clinical problem. For the remainder of this paper, stent and catheter infections will be considered together (CAUTI).

In order to develop effective mechanisms to decrease the incidence of CAUTI, the underlying sequence of events resulting in the development of infection need to be understood. When sterile urinary catheters and stents are inserted into the human body, components in urine, blood, or surrounding tissue, such as polysaccharides, ions, and glycoproteins, get deposited on the surface of the device [1,3] forming a urinary conditioning film. Considering that the conditioning film components have varying physical characteristics, their deposition alters the surface properties of the implants, allowing various planktonic bacteria to adhere to the surface via multiple mechanisms 
including electrostatic interactions and bacterial adhesins [4,5]. The initial interaction between bacteria and device surface is reversible as it is driven by weak hydrophobic and electrostatic forces [6], however, over time, the adherence becomes irreversible due to the binding of bacterial adhesins to their target molecules on the device surface as well as bacterial exopolysaccharide secretion, resulting in the formation of a biofilm. Biofilms are highly structured and actively growing bacterial communities that consist of multiple bacterial layers protected by a thick exopolysaccharide layer [6]. The presence of this thick protective layer combined with the fact that the phenotypes and metabolic functions of the embedded bacteria are modified, result in biofilms being significantly more resistant to antimicrobial drugs or disturbances than their planktonic counterparts [7] mostly due to the fact that antimicrobial agents cannot penetrate sufficiently through the exopolysaccharide layer towards the underlying bacteria as well as the strength with which it holds the community together. [8] As such, bacteria in a well-established biofilm have been shown to survive in antibiotic concentrations up to 1000 -fold higher than the minimal inhibitory concentration for their planktonic counterparts [6,9]. As the biofilm becomes more developed, the expansion of the biofilm to "unpopulated" areas of the stents is facilitated by the detachment of bacterial cells from the biofilm followed by subsequent conversion back into planktonic, or free-swimming, state. Diffusion of these planktonic bacteria to non-colonized areas of the surface results in the initiation of new biofilm formation [10].

Device associated infections in urology are complicated by the fact that the majority of uropathogens are able to form these complex biofilm communities including both Gram positive and Gram negative bacteria, as well as yeast [9,11-14]. The most commonly isolated strains associated with uropathogenic biofilms are Escherichia coli, Enterococcus faecalis, and Pseudomonas aeruginosa, E. faecalis along with Proteus mirabilis, Staphylococcus aureus and Candida tropicalis are considered to be the strongest biofilm formers among uropathogens [15]. $P$. mirabilis biofilms are further complicated by the fact that it expresses urease, an enzyme capable of hydrolyzing urea up to $10 \times$ faster than the rate of other bacterial species. This process generates ammonia, which rapidly increases the alkalinity of urine significantly, creating an environment that promotes formation of hydroxyapatite and struvite crystals, resulting in a significant encrustation of the device surface [16]. Aside from promoting further bacterial adhesion and biofilm formation, these encrustations also block the catheter lumen often resulting in complete device failure [1,6].

While improved hygiene procedures as well as regular device replacement have helped decreased the incidence of CAUTI, it has not been prevented. As such it is crucial to develop strategies that will specifically inhibit the adhesion and growth of uropathogens. While the use of prophylactic antibiotics could be an effective way to kill potential intruders of the urinary tract prior to their adhesion to the device surface, it increases the development of further resistance and will not prevent the attachment and biofilm formation of uropathogens that are already resistant. As such approaches to prevent the initial bacterial attachment to surfaces need to be developed further, as this would prevent the bacteria from being retained in the urinary tract environment, being flushed back out by normal urinary flow.

To date, several approaches have been attempted: one of the most promising approaches involves the use of antimicrobial coatings on the surface of the urinary implants [1,3,17]. To date, 
various such coatings have been designed and tested for their anti-microbial activity, resulting in highly variable success rates in preventing CAUTI. These are discussed below.

\section{Anti-Microbial Coatings to Inhibit Bacterial Growth and Adhesion}

\subsection{Antibiotic Coatings}

The use of antibiotics can be traced back to decades ago, with the sulfonamide Prontosil being the first commercially available antibiotic developed in the 1930s [18]. Ever since then, several other classes of these bacteriostatic (preventing bacterial growth) or bactericidal (killing bacteria) compounds have been discovered, each targeting different bacterial functions or growth processes [19]. Initial approaches to coat device surfaces with antibiotics such as ciprofloxacin, gentamicin, norfloxacin, and nitrofurazone included dipping the implant into organic solvents containing antibiotics followed by the evaporation of the solvent and the deposition of the drug onto the device surface [20,21]. While this method was shown to be effective for short-term implants, it was complicated by uncontrolled release profiles of the drugs resulting in the elution of initial high local concentrations that may initially damage the cells followed by concentrations that are not inhibitory. Considering that this will not kill all of the bacteria effectively, it will result in subsequent infection that will be more difficult to eradicate due to the development of resistance [22]. As such the constant elution of at least the minimal inhibitory concentration of a given antibiotic is crucial for effective antibacterial activity.

Another more recent approach to apply antibiotics to implant surfaces is to incorporate the compounds into biodegradable coatings such that the drugs are released at the rate of the coating's degradation [3]. Examples of such coatings are PVP (poly vinyl propylene), polyurethane, and calcium phosphate [3]. Similarly, enzyme-based drug elution technologies have been examined including the enzyme lipase B embedded in a polycaprolactone (PCL)-based coating containing the antibiotic gentamicin sulfate. In this elution mechanism the PCL serves as a substrate for lipase B, triggering the degradation of the coating with subsequent release of the antibiotic [3]. Despite the fact that this approach was shown to be effective for up to 16 days, it was abandoned due to problems with the biocompatibility of lipase B [3].

The combination of different antibiotics has also been shown to behave synergistically in preventing the adhesion and growth of uropathogens [23]. Using an in vivo rat model, Minardi et al. were able to demonstrate the efficacy of combining rifampin-soaked ureteral stents with intraperitoneal injection of tigecycline against the growth and biofilm formation of E. faecalis [23]. However, this method is only effective when both antibiotics are administered together, as resistance against rifampin is rapidly acquired by Gram positive bacteria when used alone [23].

\subsection{Triclosan}

Triclosan is an antimicrobial agent that has been used in consumer products for approximately 40 years [6]. It is a ubiquitous compound that affects both Gram positive and Gram negative bacteria affecting the stability of their cell walls - and it has been loaded into Foley catheters and ureteral stents [24-26]. Previous in vivo studies testing the efficacy of triclosan eluting stents in a 
rabbit model of UTI, demonstrated that triclosan-eluting stents significantly decreased the rate of P. mirabilis associated UTIs and bacterial load in comparison to non-eluting controls [26]. Moreover, Lange et al. were able to show triclosan-eluting stents to be effective in hindering bacterial adherence of uropathogenic E. coli, Klebsiella pneumoniae, and S. aureus [27]. In patients with long-term ureteral stents, the biofilm formation was too extensive and the amount of triclosan loaded into the stent was no match for the bacterial load that overwhelmed the ureteral stent within 3-6 months [28]. Although approved in many parts of the world, the triclosan eluting ureteral stent (Triumph $^{\mathrm{TM}}$, Boston Scientific Corporation, Marlborough, MA) was never FDA approved due to the potential concern that it would lead to further antibiotic resistance. Triclosan has never been shown to elicit any type of bacterial resistance - in vitro or in clinical studies [29]. In patients stented for less than 4 weeks, this stent did prove to relieve stent symptoms-giving credence to the theory that the anti-inflammatory effects of triclosan, rather than its antimicrobial effects, may be its most efficacious property in the urinary tract [30,31].

\subsection{Silver}

Silver is an effective broad-spectrum antimicrobial agent at low concentrations [2]. Implants composed from metallic silver have been shown to be inert prior to implantation, yet become highly reactive and ionize quickly once in contact with bodily fluids [1]. The reactive metal then generates silver ions capable of modifying bacterial cell walls and membranes, as well as inhibiting bacterial genome replication [1]. Although this type of metallic implant has been shown to be effective at preventing infections, patients are faced with significant morbidity including abdominal pain likely associated with the inflexible nature of the implant. Thus, alternatives such as silver-coated and silver-impregnated catheters have been proposed [12], however their effectiveness was found to be highly variable among studies [8]. Previous studies have found negative effects associated with the catheter materials, as silver-impregnated latex catheters were found to decrease bacterial adhesion more effectively than silver-impregnated silicone catheters [8]. Moreover, the activity of the silver particles was shown to be higher when particles are less than $10 \mathrm{~mm}$ in size, shaped triangularly rather than spherically, and are present in oxidized rather than reduced forms [4,6]. These limitations significantly complicate the design of highly effective silver-based coatings. Potential problems associated with these coatings include argyria as a result of prolonged usage [3].

\subsection{Hydrogel Coatings}

Hydrogel is a hydrophilic, cross-linked polymer capable of absorbing large volumes of liquid [32] forming a thin layer of water on the coated device surface, preventing conditioning film formation by preventing the deposition of components including fibrinogen and platelets, that may facilitate bacterial adhesion [20]. In addition, due to the smooth and lubricious nature of hydrogel-coated catheters, these implants have been associated with less urethral irritation and inflammation [33]. In one study, hydrogel-coated silicone catheters resulted in $90 \%$ less E. faecalis adherence than uncoated catheters [33], although results from other studies were highly variable. In addition, a recent study by Elwood et al. has suggested that the presence of a conditioning film on catheter 
surfaces do not increase bacterial adhesion and colonization of urinary device surfaces compared to unconditioned controls [34].

\subsection{Polyvinylpyrrolidone (PVP)}

Similar to hydrogel, polyvinylpyrrolidone (PVP) is a hydrophilic, water-soluble polymer, capable of absorbing $40 \%$ of its weight with water [35,36]. Its excellent lubricant properties results in a soft, smooth and non-adhesive implant surface that facilitates device implantation, and was shown to reduce the adherence of E. faecalis and device encrustation in vitro, in comparison to uncoated polyurethane catheters [36].

\subsection{Heparin Coating}

Heparin is a highly sulfated glycosaminoglycan, often used as an anticoagulant with the highest negative charge density amongst all known biologic molecules [37]. Three different ways have been previously used to coat heparin on implant surfaces. These include physically adsorbing heparin, incorporating heparin into a polymer, and covalently binding heparin to implant surfaces via a spacer, with the last option being the most effective [37]. Covalently-bound heparin catheters have been shown to result in no detectable biofilm formation or encrustation for up to 6 weeks under clinical trials $[38,39]$. It is believed that the strong electronegativity of the coating repels microorganisms [37-39]. More recently, however, a study performed by Lange et al. studying bacterial adhesion to the surface of heparin-coated stents over a period of one week in vitro showed no decrease in bacterial adhesion to heparin-coated stents [27]. Although heparin coatings show great clinical performance in vascular catheters, the interaction of material and urine are not as beneficial as they are in blood making heparin a poor coating for urinary biomaterials.

\subsection{Hyaluronic Acid Coating}

Hyaluronic acid is a type of glycosaminoglycan [40]. It is an inhibitor of nucleation, growth, and aggregation of salts. Covalently bound hyaluronic acid catheters have been associated with increased hydration, decreased adsorption of proteins, and decreased bacterial adhesion [41]. While promising results were obtained in in vitro studies, the efficacy of hyaluronic acid coated stents in the clinical setting remains to be determined [41].

\subsection{Gendine}

Gendine is a novel antiseptic that contains Gentian Violet and chlorhexidine [6]. Catheters coated with this antiseptic have been shown to resist adherence of several multidrug-resistant bacteria in comparison to uncoated controls, as assessed by adhesion assays and scanning electron microscopy for visualizing biofilm formation [42]. When compared to silver hydrogel-coated catheters in a rabbit model, Gendine-coated urinary catheters were more effective at preventing bacterial colonization and urinary tract infections from E. coli, P aeruginosa, K. pneumoniae, and Candida species. Histopathologic examination showed no differences in inflammation between the silver coated catheters or the gendine antiseptic group. 


\subsection{Chitosan Coating}

Chitosan is a natural cationic, biodegradable polysaccharide and a weak polyelectrolyte $[10,43]$. It is a non-toxic biopolymer obtained via chitin deacetylation, and has broad-spectrum activity against bacteria $[10,43]$. Although its exact mechanism is unknown, it is hypothesized to result in leaky cell membranes [10]. Due to its poorly soluble nature, chitosan has had limited capabilities for its use as a catheter coating. To overcome this limitation, water-soluble quaternised chitosan derivatives, known as hydroxypropyltrimethyl ammonium chloride chitosan, has been previously tested as a novel coating [43]. Recent studies have shown quaternised chitosan-loaded polymethylmethacrylate (PMMA) to be more effective at inhibiting biofilm formation of methicillin-resistant and non-resistant $S$. epidermidis and $S$. aureus than PMMA alone or gentamicin-loaded PMMA. Interestingly, these coatings were able to down-regulate the expression of genes encoding for enzymes responsible for biofilm biosynthesis, as demonstrated by real-time PCR [43].

To further strengthen the anti-adhesive and anti-bacterial capabilities of the chitosan coating, past researchers have constructed a multilayer film by assembling chitosan heparin in a layer-by-layer strategy onto aminolyzed poly(ethylene terephthalate) (PET) films [44]. This type of multilayer coating significantly decreased bacterial adhesion and the number of viable bacteria when co-incubated with E. coli. This multilayer coating demonstrated further antibacterial activity when silver nanoparticles were also incorporated [44].

\subsection{Low-Energy Surface Acoustic Waves (SAW)}

Low-energy acoustic waves transmitted directly to indwelling devices have been shown to inhibit bacterial adhesion to the implant surface by disrupting the formation of biofilms [45]. With a portable actuator generating piezoelectric vibrations between frequencies of 100 to $200 \mathrm{kHz}$, acoustic waves can be delivered to the surface of catheters [45]. Although there is no actual coating placed on the surface of implants, the waves cover the entire surface, generating a virtual vibrating coat [45]. This type of treatment has been shown to significantly inhibit biofilm formation of E. coli, P. mirabilis, and Candida albicans in comparison to non-vibrating controls [45]. The results have been confirmed using an in vivo rabbit model, where the treatment maintained sterility in urine for over one week in comparison to only 2 days in control animals [6,45]. However, to maintain the vibrating coating, elastic waves would need to be continuously delivered throughout the implantation process, which may be complicated by the fact that patients would be required to carry a portable actuator with them at times [45].

\subsection{Salicylic Acid-Releasing Polyurethane Acrylate Polymers}

Salicylic acid is a metabolite of aspirin, and has been known to have various effects on bacteria [46]. A recently studied salicylic-acid eluting coating involves ultraviolet-cured polyurethane acrylate polymer containing salicyl acrylate [1]. Under aqueous environments, the polymer hydrolyzes and releases salicylic acid, leaving the backbone intact. The rate of salicylic acid release is dependent upon the composition of the polymer, and the release of such acid was shown to inhibit biofilm 
formation of $S$. epidermidis, Bacillus subtilis, E. coli, P. aeruginosa, as well as $S$. aureus [1]. The effect was measured via a MBEC assay biofilm multi-peg growth system and bioluminescence monitoring, and the coated catheters were shown to be effective against biofilm formation of E. coli in vitro up to 5 days [1]. Although the mechanism is currently unknown, it is believed to hinder bacterial quorum sensing [1].

\subsection{Antimicrobial Peptides Conjugated to Co-Polymer Brushes}

Another more recent coating being studied in other areas of medicine includes antimicrobial peptides (AMPs) coatings [47]. These peptides are generally short, comprised of 10 to 50 residues of mainly lysine and arginine, making the peptide cationic [47,48]. Although the mechanism of action is not well understood, AMPs are believed to mainly disrupt the bacterial cell wall and cell membrane as well as affect DNA or RNA replication, protein synthesis, and many other bacterial processes [47]. Since AMPs are broad-spectrum and most likely target many processes rather than

just one specific target, the likelihood of bacteria generating resistance against the peptides are relatively low, making them excellent antimicrobial agents [49]. In a recent study, antimicrobial peptides were coated on titanium implants via conjugation with hydrophilic polymer brushes [47]. These tethered peptides were capable of inhibiting bacterial growth both in vitro and in vivo using rat models [47]. During in vivo models, not only did the peptides show antibacterial effect, they also appeared to possess wound-healing effects [50]. Indeed, despite the promising results seen with AMPs, there are still caveats associated with the coatings. These include potential local toxicity, $\mathrm{pH}$ sensitivity, sensitization and allergy after repeated exposures, susceptibility to proteolysis, and the high cost of synthesis [49]. Despite this, the fact that AMPs can be easily modified to decrease these effects, their effectiveness as a catheter coating warrants further investigation as a novel approach to prevent bacterial adhesion and associated infection.

\section{Conclusions}

Although numerous antimicrobial coatings have been applied to ureteral stents and urinary catheters in an attempt to reduce CAUTI resulting from bacterial growth and adhesion on the medical devices, few of them of been shown to be fully effective. One of the major complications associated with antibiotic based coatings is the development of resistance. More novel antimicrobial agents, such as low-energy acoustic waves and antimicrobial peptides, are capable of avoiding this complication. However, in turn they may face other problems such as high cost of delivery or synthesis and potential toxicity. Recent studies have attempted to combine several antimicrobial agents into a single coating, such as the incorporation of several antibiotics into one coating or the multilayered heparin-chitosan film. However, further scientific and clinical studies are required before the ideal antimicrobial coating can be identified and introduced into clinical practice.

\section{Author Contributions}

Each author has contributed to the research of this review article and drafting and finalizing this manuscript. 


\section{Conflicts of Interest}

The authors declare no conflict of interest.

\section{References}

1. Nowatzki, P.J.; Koepsel, R.R.; Stoodley, P.; Min, K.; Harper, A.; Murata, H.; Donfack, J.; Hortelano, E.R.; Ehrlich, G.D.; Russell, A.J. Salicylic acid-releasing polyurethane acrylate polymers as anti-biofilm urological catheter coatings. Acta Biomater. 2012, 8, 1869-1880.

2. Saint, S.; Meddings, J.A.; Calfee, D.; Kowalski, C.P.; Krein, S.L. Catheter-associated urinary tract infection and the Medicare rule changes. Ann. Intern. Med. 2009, 150, 877-884.

3. Dave, R.N.; Joshi, H.M.; Venugopalan, V.P. Novel biocatalytic polymer-based antimicrobial coatings as potential ureteral biomaterial: Preparation and in vitro performance evaluation. Antimicrob. Agents Chemother. 2011, 55, 845-853.

4. Gristina, A.G.; Giridhar, G.; Gabriel, B.L.; Naylor, P.T.; Myrvik, Q.N. Cell biology and molecular mechanisms in artificial device infections. Int. J. Artif. Organs 1993, 16, 755-763.

5. Stickler, D.; Ganderton, L.; King, J.; Nettleton, J.; Winters, C. Proteus mirabilis biofilms and the encrustation of urethral catheters. Urol. Res. 1993, 21, 407-411.

6. Tenke, P.; Koves, B.; Nagy, K.; Hultgren, S.J.; Mendling, W.; Wullt, B.; Grabe, M.; Wagenlehner, F.M.; Cek, M.; Pickard, R.; et al. Update on biofilm infections in the urinary tract. World J. Urol. 2012, 30, 51-57.

7. Winson, L. Catheterization: A need for improved patient management. Br. J. Nurs. 1997, 6, 1229-1232, 1234, 1251-1252.

8. Hoiby, N.; Ciofu, O.; Johansen, H.K.; Song, Z.J.; Moser, C.; Jensen, P.O.; Molin, S.; Givskov, M.; Tolker-Nielsen, T.; Bjarnsholt, T. The clinical impact of bacterial biofilms. Int. J. Oral Sci. 2011, 3, 55-65.

9. Stewart, P.S.; Costerton, J.W. Antibiotic resistance of bacteria in biofilms. Lancet 2001, 358, 135-138.

10. Arciola, C.R.; Campoccia, D.; Speziale, P.; Montanaro, L.; Costerton, J.W. Biofilm formation in Staphylococcus implant infections. A review of molecular mechanisms and implications for biofilm-resistant materials. Biomaterials 2012, 33, 5967-5982.

11. Rudrappa, T.; Quinn, W.J.; Stanley-Wall, N.R.; Bais, H.P. A degradation product of the salicylic acid pathway triggers oxidative stress resulting in down-regulation of Bacillus subtilis biofilm formation on Arabidopsis thaliana roots. Planta 2007, 226, 283-297.

12. Prithiviraj, B.; Bais, H.P.; Jha, A.K.; Vivanco, J.M. Staphylococcus aureus pathogenicity on Arabidopsis thaliana is mediated either by a direct effect of salicylic acid on the pathogen or by SA-dependent, NPR1-independent host responses. Plant J. 2005, 42, 417-432.

13. Alem, M.A.; Douglas, L.J. Effects of aspirin and other nonsteroidal anti-inflammatory drugs on biofilms and planktonic cells of Candida albicans. Antimicrob. Agents Chemother. 2004, 48, 41-47.

14. Stepanovic, S.; Vukovic, D.; Jesic, M.; Ranin, L. Influence of acetylsalicylic acid (aspirin) on biofilm production by Candida species. J. Chemother. 2004, 16, 134-138. 
15. Hola, V.; Ruzicka, F.; Horka, M. Microbial diversity in biofilm infections of the urinary tract with the use of sonication techniques. FEMS Immunol. Med. Microbiol. 2010, 59, 525-528.

16. Morris, N.S.; Stickler, D.J.; McLean, R.J. The development of bacterial biofilms on indwelling urethral catheters. World J. Urol. 1999, 17, 345-350.

17. Syed, M.A.; Manzoor, U.; Shah, I.; Bukhari, S.H. Antibacterial effects of Tungsten nanoparticles on the Escherichia coli strains isolated from catheterized urinary tract infection (UTI) cases and Staphylococcus aureus. New Microbiol. 2010, 33, 329-335.

18. Klee, P.; Römer, H. Prontosil for streptococcal infections (in German). Dtsch. Med. Wschr. 1935, 61, 253-255.

19. Kimang'a, A.N. A situational analysis of antimicrobial drug resistance in Africa: Are we losing the battle? Ethiopian J. Health Sci. 2012, 22, 135-143.

20. Noimark, S.; Dunnill, C.W.; Wilson, M.; Parkin, I.P. The role of surfaces in catheter-associated infections. Chem. Soc.Rev. 2009, 38, 3435-3448.

21. Reid, G.; Sharma, S.; Advikolanu, K.; Tieszer, C.; Martin, R.A.; Bruce, A.W. Effects of ciprofloxacin, norfloxacin, and ofloxacin on in vitro adhesion and survival of Pseudomonas aeruginosa AK1 on urinary catheters. Antimicrob. Agents Chemother. 1994, 38, 1490-1495.

22. Walder, B.; Pittet, D.; Tramer, M.R. Prevention of bloodstream infections with central venous catheters treated with anti-infective agents depends on catheter type and insertion time: Evidence from a meta-analysis. Infect. Control Hosp. Epidemiol. 2002, 23, 748-756.

23. Minardi, D.; Cirioni, O.; Ghiselli, R.; Silvestri, C.; Mocchegiani, F.; Gabrielli, E.; d'Anzeo, G.; Conti, A.; Orlando, F.; Rimini, M.; et al. Efficacy of tigecycline and rifampin alone and in combination against Enterococcus faecalis biofilm infection in a rat model of ureteral stent. J. Surg. Res. 2012, 176, 1-6.

24. Stickler, D.J.; Jones, G.L.; Russell, A.D. Control of encrustation and blockage of Foley catheters. Lancet 2003, 361, 1435-1437.

25. Chew, B.H.; Cadieux, P.A.; Reid, G.; Denstedt, J.D. In-vitro activity of triclosan-eluting ureteral stents against common bacterial uropathogens. J. Endourol. 2006, 20, 949-958.

26. Cadieux, P.A.; Chew, B.H.; Knudsen, B.E.; Dejong, K.; Rowe, E.; Reid, G.; Denstedt, J.D. Triclosan loaded ureteral stents decrease proteus mirabilis 296 infection in a rabbit urinary tract infection model. J. Urol. 2006, 175, 2331-2335.

27. Lange, D.; Elwood, C.N.; Choi, K.; Hendlin, K.; Monga, M.; Chew, B.H. Uropathogen interaction with the surface of urological stents using different surface properties. J. Urol. 2009, $182,1194-1200$.

28. Cadieux, P.A.; Chew, B.H.; Nott, L.; Seney, S.; Elwood, C.N.; Wignall, G.R.; Goneau, L.W.; Denstedt, J.D. Use of triclosan-eluting ureteral stents in patients with long-term stents. J. Endourol. 2009, 23, 1187-1194.

29. Wignall, G.R.; Goneau, L.W.; Chew, B.H.; Denstedt, J.D.; Cadieux, P.A. The effects of triclosan on uropathogen susceptibility to clinically relevant antibiotics. J. Endourol. 2008, 22, 2349-2356.

30. Wallet, M.A.; Calderon, N.; Alonso, T.R.; Choe, C.S.; Catalfamo, D.; Lalane, C.J.; Neiva, K.G.; Panagakos, F.; Wallet, S.M. Triclosan alters antimicrobial and inflammatory responses of epithelial cells. Oral Dis. 2013, 19, 296-302. 
31. Sreenivasan, P.K.; Gaffar, A. Antibacterials as anti-inflammatory agents: Dual action agents for oral health. Antonie Leeuwenhoek 2008, 93, 227-239.

32. Nakagawa, N.; Yashiro, N.; Nakajima, Y.; Barnhart, W.H.; Wakabayashi, M. Hydrogel-coated glide catheter: Experimental studies and initial clinical experience. Am. J. Roentgenol. 1994, $163,1227-1229$.

33. Desai, D.G.; Liao, K.S.; Cevallos, M.E.; Trautner, B.W. Silver or nitrofurazone impregnation of urinary catheters has a minimal effect on uropathogen adherence. J. Urol. 2010, 184, $2565-2571$.

34. Elwood, C.N.; Lo, J.; Chou, E.; Crowe, A.; Arsovska, O.; Adomat, H.; Miyaoka, R.; Tomlinson-Guns, E.; Monga, M.; Chew, B.H.; et al. Understanding urinary conditioning film components on ureteral stents: Profiling protein components and evaluating their role in bacterial colonization. Biofouling 2013, 29, 1115-1122.

35. Francois, P.; Vaudaux, P.; Nurdin, N.; Mathieu, H.J.; Descouts, P.; Lew, D.P. Physical and biological effects of a surface coating procedure on polyurethane catheters. Biomaterials 1996, 17, 667-678.

36. Tunney, M.M.; Gorman, S.P. Evaluation of a poly(vinyl pyrollidone)-coated biomaterial for urological use. Biomaterials 2002, 23, 4601-4608.

37. Schierholz, J.M.; Beuth, J.; Konig, D.; Nurnberger, A.; Pulverer, G. Antimicrobial substances and effects on sessile bacteria. Zent. Bakteriol. 1999, 289, 165-177.

38. Riedl, C.R.; Witkowski, M.; Plas, E.; Pflueger, H. Heparin coating reduces encrustation of ureteral stents: A preliminary report. Int. J. Antimicrob. Agents 2002, 19, 507-510.

39. Tenke, P.; Riedl, C.R.; Jones, G.L.; Williams, G.J.; Stickler, D.; Nagy, E. Bacterial biofilm formation on urologic devices and heparin coating as preventive strategy. Int. J. Antimicrob. Agents 2004, 23, S67-S74.

40. Kitamura, T.; Zerwekh, J.E.; Pak, C.Y. Partial biochemical and physicochemical characterization of organic macromolecules in urine from patients with renal stones and control subjects. Kidney Int. 1982, 21, 379-386.

41. Choong, S.K.; Wood, S.; Whitfield, H.N. A model to quantify encrustation on ureteric stents, urethral catheters and polymers intended for urological use. BJU Int. 2000, 86, 414-421.

42. Hachem, R.; Reitzel, R.; Borne, A.; Jiang, Y.; Tinkey, P.; Uthamanthil, R.; Chandra, J.; Ghannoum, M.; Raad, I. Novel antiseptic urinary catheters for prevention of urinary tract infections: Correlation of in vivo and in vitro test results. Antimicrob. Agents Chemother. 2009, 53, 5145-5149.

43. Tan, H.; Peng, Z.; Li, Q.; Xu, X.; Guo, S.; Tang, T. The use of quaternised chitosan-loaded PMMA to inhibit biofilm formation and downregulate the virulence-associated gene expression of antibiotic-resistant staphylococcus. Biomaterials 2012, 33, 365-377.

44. Fu, J.; Ji, J.; Yuan, W.; Shen, J. Construction of anti-adhesive and antibacterial multilayer films via layer-by-layer assembly of heparin and chitosan. Biomaterials 2005, 26, 6684-6692.

45. Hazan, Z.; Zumeris, J.; Jacob, H.; Raskin, H.; Kratysh, G.; Vishnia, M.; Dror, N.; Barliya, T.; Mandel, M.; Lavie, G. Effective prevention of microbial biofilm formation on medical devices by low-energy surface acoustic waves. Antimicrob. Agents Chemother. 2006, 50, 4144-4152. 
46. Price, C.T.; Lee, I.R.; Gustafson, J.E. The effects of salicylate on bacteria. Int. J. Biochem. Cell Biol. 2000, 32, 1029-1043.

47. Gao, G.; Yu, K.; Kindrachuk, J.; Brooks, D.E.; Hancock, R.E.; Kizhakkedathu, J.N. Antibacterial surfaces based on polymer brushes: Investigation on the influence of brush properties on antimicrobial peptide immobilization and antimicrobial activity. Biomacromolecules 2011, 12, 3715-3727.

48. Gao, G.; Lange, D.; Hilpert, K.; Kindrachuk, J.; Zou, Y.; Cheng, J.T.; Kazemzadeh-Narbat, M.; Yu, K.; Wang, R.; Straus, S.K.; et al. The biocompatibility and biofilm resistance of implant coatings based on hydrophilic polymer brushes conjugated with antimicrobial peptides. Biomaterials 2011, 32, 3899-3909.

49. Glinel, K.; Thebault, P.; Humblot, V.; Pradier, C.M.; Jouenne, T. Antibacterial surfaces developed from bio-inspired approaches. Acta Biomater. 2012, 8, 1670-1684.

50. Lange, D. The University of British Columbia, Vancouver, Canada. Unpublished data, 2014. 


\section{Chapter 10}

\section{In Vitro Antibiofilm Efficacies of Different Antibiotic Combinations with Zinc Sulfate against Pseudomonas aeruginosa Recovered from Hospitalized Patients with Urinary Tract Infection}

\section{Walid Elkhatib and Ayman Noreddin}

Abstract: Urinary tract infections (UTIs) are a serious healthcare dilemma influencing millions of patients every year and represent the second most frequent type of body infection. Pseudomonas aeruginosa is a multidrug-resistant pathogen causing numerous chronic biofilm-associated infections including urinary tract, nosocomial, and medical devices-related infections. In the present study, the biofilm of $P$. aeruginosa CCIN34519, recovered from inpatients with UTIs, was established on polystyrene substratum and scanning electron microscopy (SEM) and was utilized for visualization of the biofilm. A previously described in vitro system for real-time monitoring of biofilm growth/inhibition was utilized to assess the antimicrobial effects of ciprofloxacin, levofloxacin, moxifloxacin, norfloxacin, ertapenem, ceftriaxone, gentamicin, and tobramycin as single antibiotics as well as in combinations with zinc sulfate $(2.5 \mathrm{mM})$ against $P$. aeruginosa CCIN34519 biofilm. Meanwhile, minimum inhibitory concentrations (MICs) at $24 \mathrm{~h}$ and mutant prevention concentrations (MPCs) at $96 \mathrm{~h}$ were determined for the aforementioned antibiotics. The real-time monitoring data revealed diverse responses of $P$. aeruginosa CCIN34519 biofilm to the tested antibiotic-zinc sulfate combinations with potential synergisms in cases of fluoroquinolones (ciprofloxacin, levofloxacin, moxifloxacin, and norfloxacin) and carbapenem (ertapenem) as demonstrated by reduced MIC and MPC values. Conversely, considerable antagonisms were observed with cephalosporin (ceftriaxone) and aminoglycosides (gentamicin, and tobramycin) as shown by substantially increased MICs and MPCs values. Further deliberate in vivo investigations for the promising synergisms are required to evaluate their therapeutic potentials for treatment of UTIs caused by $P$. aeruginosa biofilms as well as for developing preventive strategies.

Reprinted from Antibiotics. Cite as: Elkhatib, W.; Noreddin, A. In Vitro Antibiofilm Efficacies of Different Antibiotic Combinations with Zinc Sulfate against Pseudomonas aeruginosa Recovered from Hospitalized Patients with Urinary Tract Infection. Antibiotics 2014, 3, 64-84.

\section{Introduction}

Biofilm associated infections tend to be reluctant and difficult to eradicate [1]. The increased antibiotic resistance of biofilms was generally attributed to the biofilm-associated patterns of gene expression, slow growth rate, and substantially diminished antimicrobial diffusion within the biofilm [2]. Biofilms are considered relevant to the clinical settings because they play key roles in 
the ability of biofilm-ensconced bacteria to tolerate relatively high therapeutic doses of antibiotics and persist in chronic infections [3,4]. Urinary tract infections (UTIs) are one of the most common bacterial infections affecting humans all through their life period [5,6]. UTIs are responsible for more than eight million visits to clinicians and more than two million admissions to the emergency rooms in the United States annually [6,7]. Furthermore, UTIs are the most common urological disease in the United States, with a financial burden on the healthcare system exceeding $\$ 3.5$ billion annually [8].

Pseudomonas aeruginosa is an important opportunistic human pathogen and is eminent for chronic infections including medical device-associated infections as well as urinary tract infections [9]. The urinary tract infection (UTI) caused by $P$. aeruginosa is a serious health problem affecting millions of people worldwide each year and catheterization of the urinary tract is one of the most common predisposing factors to such infections [6]. Indisputably, biofilm formation was considered to be the key feature of $P$. aeruginosa survival in chronic infections, and extracellular matrices of the biofilms provide structural scaffold and protective barricade against antibiotics [10,11]. In $P$. aeruginosa biofilm, the efficacy of the antimicrobial chemotherapy may be attenuated not only by deficiency of antibiotics access caused by the sheltered bacteria within the exopolysaccharide alginate matrix but also by emergence of the mutant subpopulations [12].

Fluoroquinolones, $\beta$-lactams and aminoglycosides are the main classes of antibiotics used for treatment of $P$. aeruginosa infections. Unfortunately, the multi-drug resistant isolates of $P$. aeruginosa which patients are often exposed to in hospital settings are resistant to one or more of these antibiotic classes [13-17]. Selective pressure due to excessive exposure of bacteria to antibiotics is generally the origin of such high incidence of resistance in the hospitals environment. Intensive care units (ICU) and long-term care facilities are also notorious worldwide for harboring multi-drug and pan-drug resistant $P$. aeruginosa strains $[17,18]$. Owing to the unmet medical needs of novel antimicrobial agents $[19,20]$, the use of combination therapy has gained attention as an option strategy for combating $P$. aeruginosa biofilm associated infections [21-23]. Zinc is a vital trace element required for virtually all forms of life [24]. It is also essential for all bacteria, but surplus amounts of the metal can possess toxic effects on them [25] and zinc chelation with nitroxoline, a urinary antibiotic, induced the dispersal of $P$. aeruginosa biofilms [26]. In this context, the objective of the current study was to evaluate the antimicrobial interactions of different fluoroquinolones, carbapenem, cephalosporin, and aminoglycosides with zinc sulfate against biofilm of the uro-pathogen, P. aeruginosa CCIN34519, through implementation of in vitro real-time monitoring system.

\section{Results and Discussion}

\subsection{Scanning Electron Microscopy of the Biofilm}

Variable pressure scanning electron microscopy was used for visualization of the biofilm on polystyrene plates after $12 \mathrm{~h}$ of incubation in absence of physical distortion or gold coating of the biofilm. The SEM demonstrated a well-established P. aeruginosa CCIN34519 biofilm as shown in Figure 1 . 
Figure 1. Colored scanning electron micrograph illustrating the appearance of P. aeruginosa CCIN34519 biofilm on polystyrene substratum.

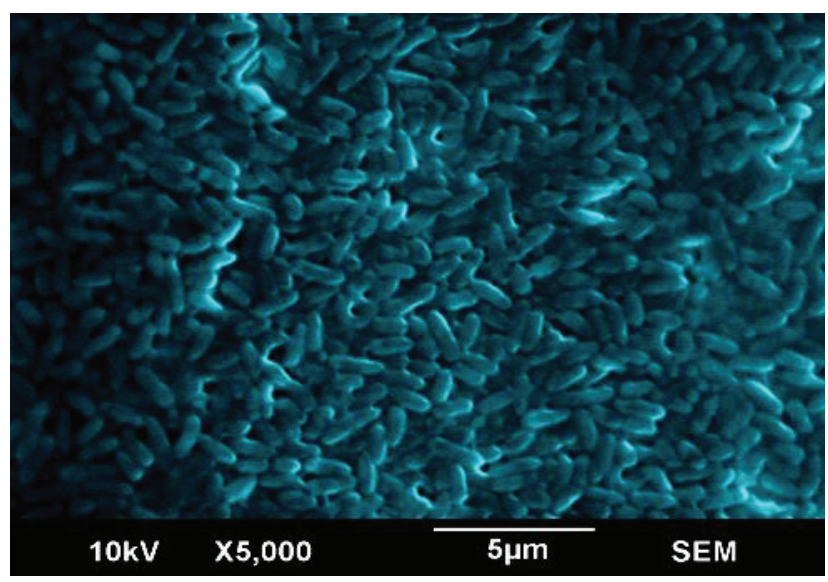

\subsection{Real-Time Monitoring of P. aeruginosa CCIN34519 Biofilm}

The effect of different concentrations of ciprofloxacin $(0.1-1.6 \mu \mathrm{g} / \mathrm{mL})$ alone on $P$. aeruginosa CCIN34519 biofilm as compared to the control (cation adjusted Mueller Hinton II; CAMH broth) is shown in Figure 2a. The minimum inhibitory concentration (MIC) and mutant prevention concentration (MPC) of ciprofloxacin against $P$. aeruginosa CCIN34519 biofilm, as determined by kinetic measurements by means of the Bioscreen $\mathrm{C}$, were $0.8 \mu \mathrm{g} / \mathrm{mL}$ at $24 \mathrm{~h}$ and $1.6 \mu \mathrm{g} / \mathrm{mL}$ at $96 \mathrm{~h}$, respectively. Real-time monitoring data showed that ciprofloxacin alone inhibited the biofilm growth for $4,8,20$, and $38 \mathrm{~h}$ with the tested concentration levels of $0.1,0.2,0.4$, and $0.8 \mu \mathrm{g} / \mathrm{mL}$, respectively. For the untreated biofilm, microbial growth from $P$. aeruginosa CCIN34519 biofilm exponentially increased and reached the peak at $10 \mathrm{~h}$ of incubation, followed by a gradual decline for up to $35 \mathrm{~h}$, and finally maintained the plateau until the end of the experiment (Figure $2 \mathrm{a}$ ). In this study, zinc sulfate concentration $(2.5 \mathrm{mM})$ was selected as the highest concentration that ho inhibitory effect on the growth of $P$. aeruginosa CCIN34519 biofilm as shown in Figure $2 \mathrm{~b}$. In the presence of zinc sulfate $(2.5 \mathrm{mM})$, the MIC and MPC of ciprofloxacin against $P$. aeruginosa CCIN34519 biofilm decreased to $0.2 \mu \mathrm{g} / \mathrm{mL}$ at $24 \mathrm{~h}$ and $0.4 \mu \mathrm{g} / \mathrm{mL}$ at $96 \mathrm{~h}$, respectively. Furthermore, ciprofloxacin could inhibit the biofilm growth for 17 and $60 \mathrm{~h}$ at concentration levels of 0.1 , and $0.2 \mu \mathrm{g} / \mathrm{mL}$, respectively (Figure $2 \mathrm{~b}$ ).

The MIC and MPC of levofloxacin against $P$. aeruginosa CCIN34519 biofilm were $4.0 \mu \mathrm{g} / \mathrm{mL}$ and $8.0 \mu \mathrm{g} / \mathrm{mL}$, respectively, and levofloxacin alone inhibited the biofilm growth for 18,22 , and $30 \mathrm{~h}$ with the concentration levels of $1.0,2.0$, and $4.0 \mu \mathrm{g} / \mathrm{mL}$, respectively (Figure 3a). On the other hand, the MIC and MPC of levofloxacin, in the presence of zinc sulfate $(2.5 \mathrm{mM})$, were curtailed to $0.25 \mu \mathrm{g} / \mathrm{mL}$ and $0.5 \mu \mathrm{g} / \mathrm{mL}$, respectively. Furthermore, levofloxacin inhibited the biofilm growth for 20 and $36 \mathrm{~h}$ at concentration levels of 0.13 , and $0.25 \mu \mathrm{g} / \mathrm{mL}$, respectively (Figure $3 \mathrm{~b}$ ). 
Figure 2. Real-time data showing the effect of (a) Ciprofloxacin alone $(0.1-1.6 \mu \mathrm{g} / \mathrm{mL})$ and (b) Ciprofloxacin $(0.1-1.6 \mu \mathrm{g} / \mathrm{mL})$ in combination with zinc sulfate $(2.5 \mathrm{mM})$ on $P$. aeruginosa CCIN34519 biofilm as monitored by the Bioscreen C over $96 \mathrm{~h}$. Control represents cation adjusted Mueller Hinton II broth (CAMH) while, $\mathrm{ZnSO}_{4}$ indicates CAMH broth supplemented with $2.5 \mathrm{mM}$ zinc sulfate.

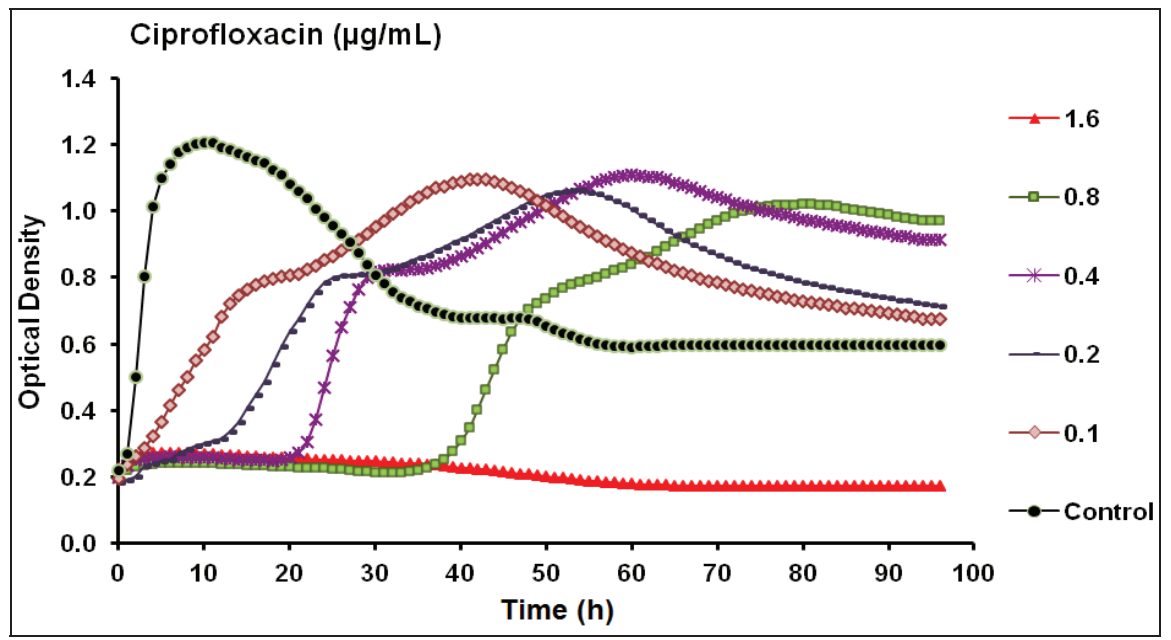

(a)

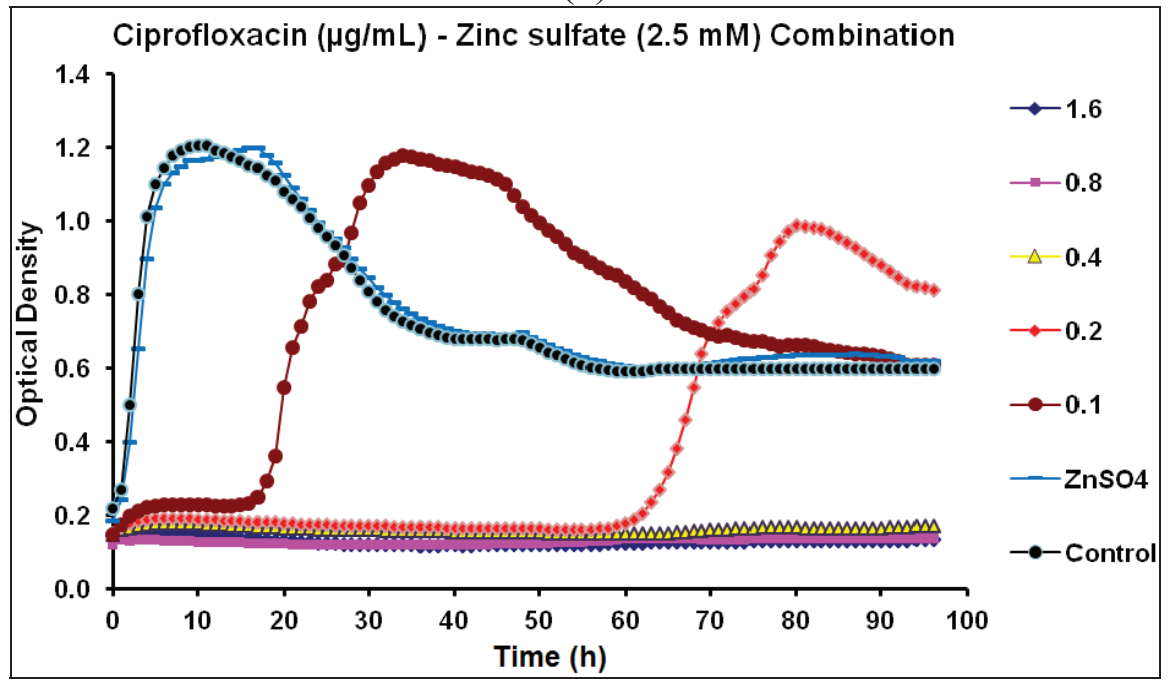

(b)

For moxifloxacin, the MIC and MPC of against $P$. aeruginosa CCIN34519 biofilm, as determined by kinetic measurements, were $16 \mu \mathrm{g} / \mathrm{mL}$ and $32 \mu \mathrm{g} / \mathrm{mL}$, respectively. Real-time monitoring data demonstrated that moxifloxacin alone suppressed the biofilm growth for 15 and $50 \mathrm{~h}$ with the tested concentration levels of 8 and $16 \mu \mathrm{g} / \mathrm{mL}$, respectively (Figure $4 \mathrm{a}$ ). In the presence of zinc sulfate $(2.5 \mathrm{mM})$, the MIC and MPC of moxifloxacin against $P$. aeruginosa CCIN34519 biofilm reduced to $2.0 \mu \mathrm{g} / \mathrm{mL}$ and $8.0 \mu \mathrm{g} / \mathrm{mL}$, respectively. Furthermore, moxifloxacin could inhibit the biofilm growth for up to 20,32 and $58 \mathrm{~h}$ at concentration levels of $1.0,2.0$ and $4.0 \mu \mathrm{g} / \mathrm{mL}$, respectively (Figure 4b). 
Figure 3. Real-time data showing the effect of (a) Levofloxacin alone $(0.13-8.00 \mu \mathrm{g} / \mathrm{mL})$ and (b) Levofloxacin $(0.13-8.00 \mu \mathrm{g} / \mathrm{mL})$ in combination with zinc sulfate $(2.5 \mathrm{mM})$ on P. aeruginosa CCIN34519 biofilm as monitored by the Bioscreen C over $96 \mathrm{~h}$. Control represents cation adjusted Mueller Hinton II broth (CAMH) while, $\mathrm{ZnSO}_{4}$ indicates CAMH broth supplemented with $2.5 \mathrm{mM}$ zinc sulfate.

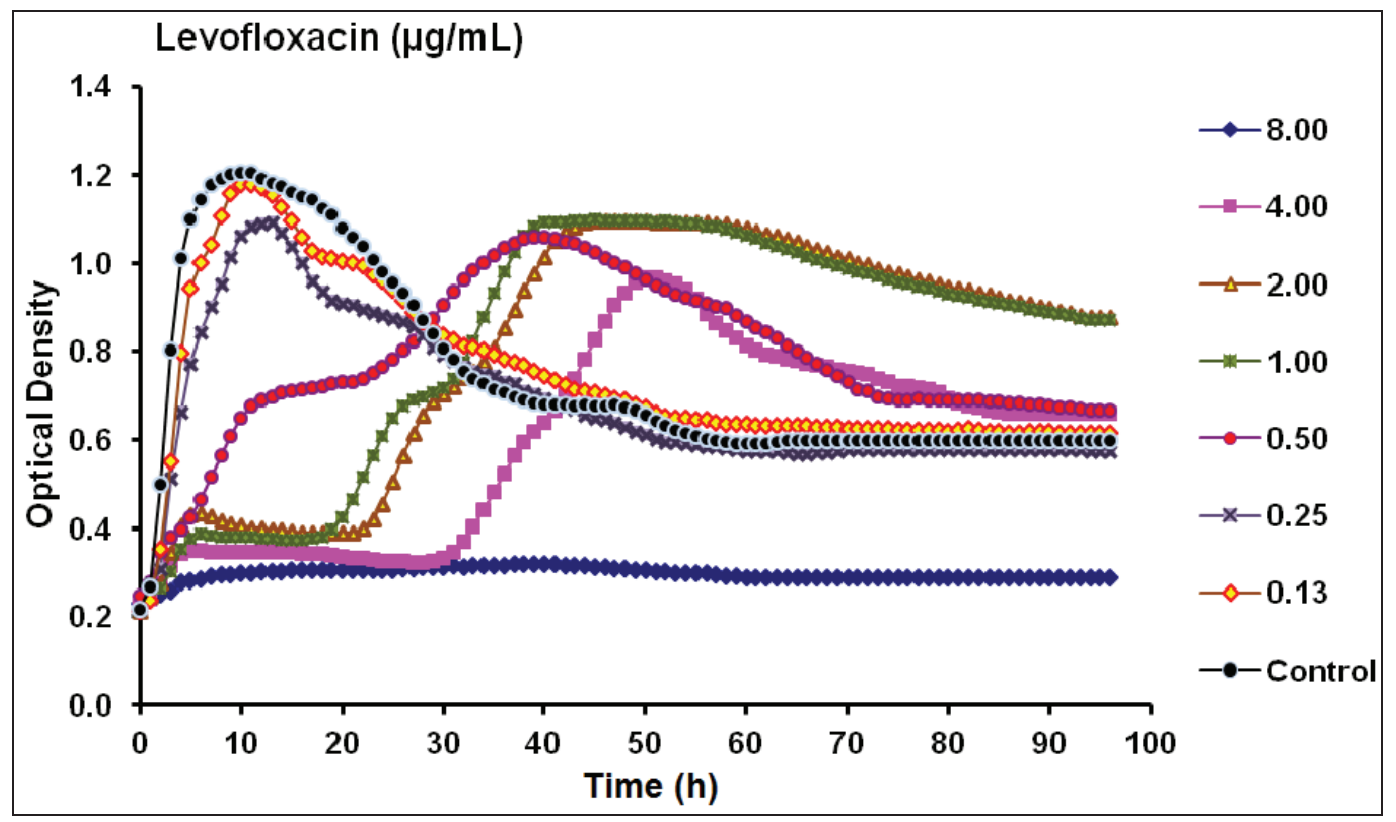

(a)

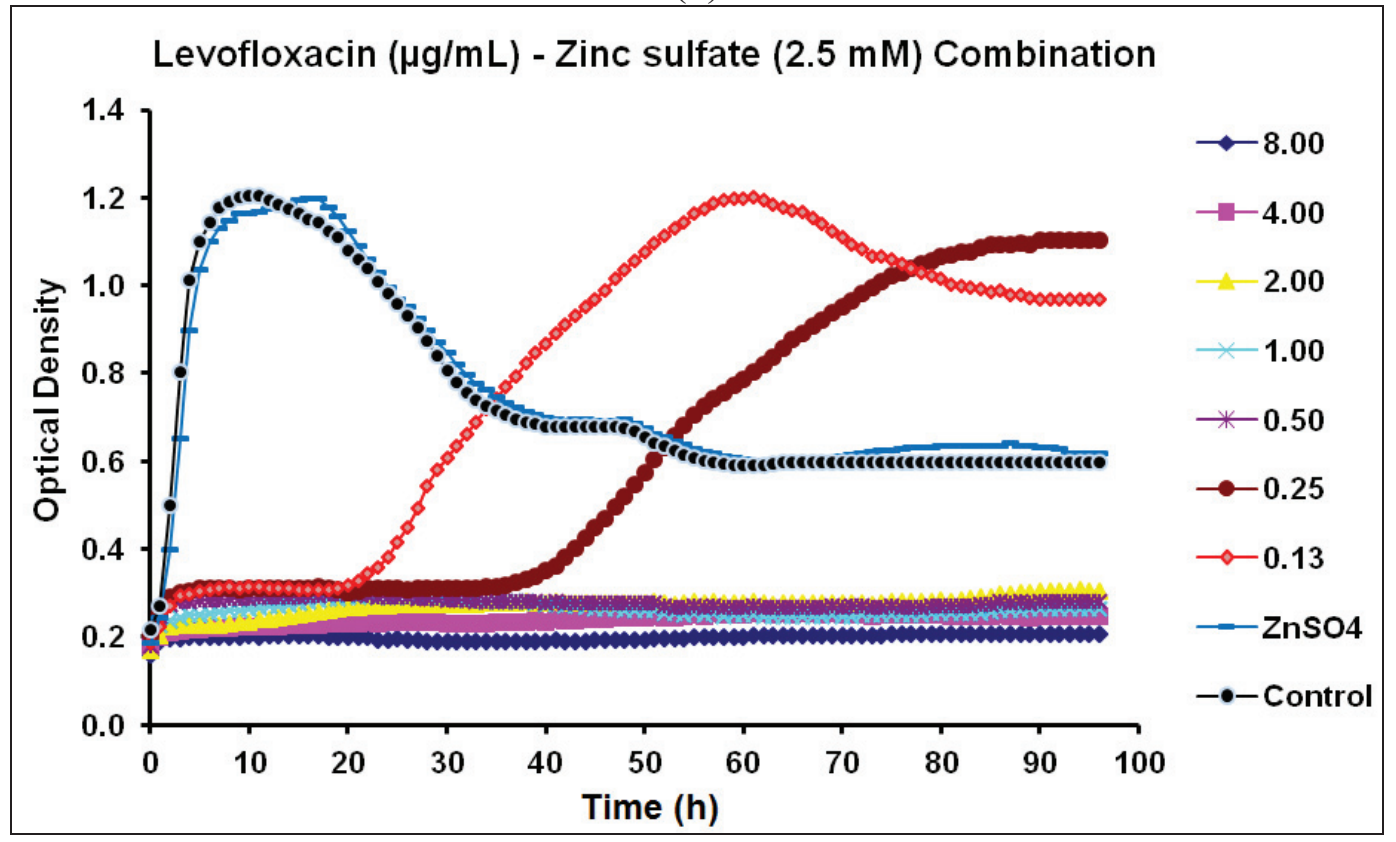

(b) 
Figure 4. Real-time data showing the effect of (a) Moxifloxacin alone (1.0-32.0 $\mu \mathrm{g} / \mathrm{mL})$ and (b) Moxifloxacin (1.0-32.0 $\mu \mathrm{g} / \mathrm{mL})$ in combination with zinc sulfate $(2.5 \mathrm{mM})$ on $P$. aeruginosa CCIN34519 biofilm as monitored by the Bioscreen C over $96 \mathrm{~h}$. Control represents cation adjusted Mueller Hinton II broth (CAMH) while, $\mathrm{ZnSO}_{4}$ indicates CAMH broth supplemented with $2.5 \mathrm{mM}$ zinc sulfate.

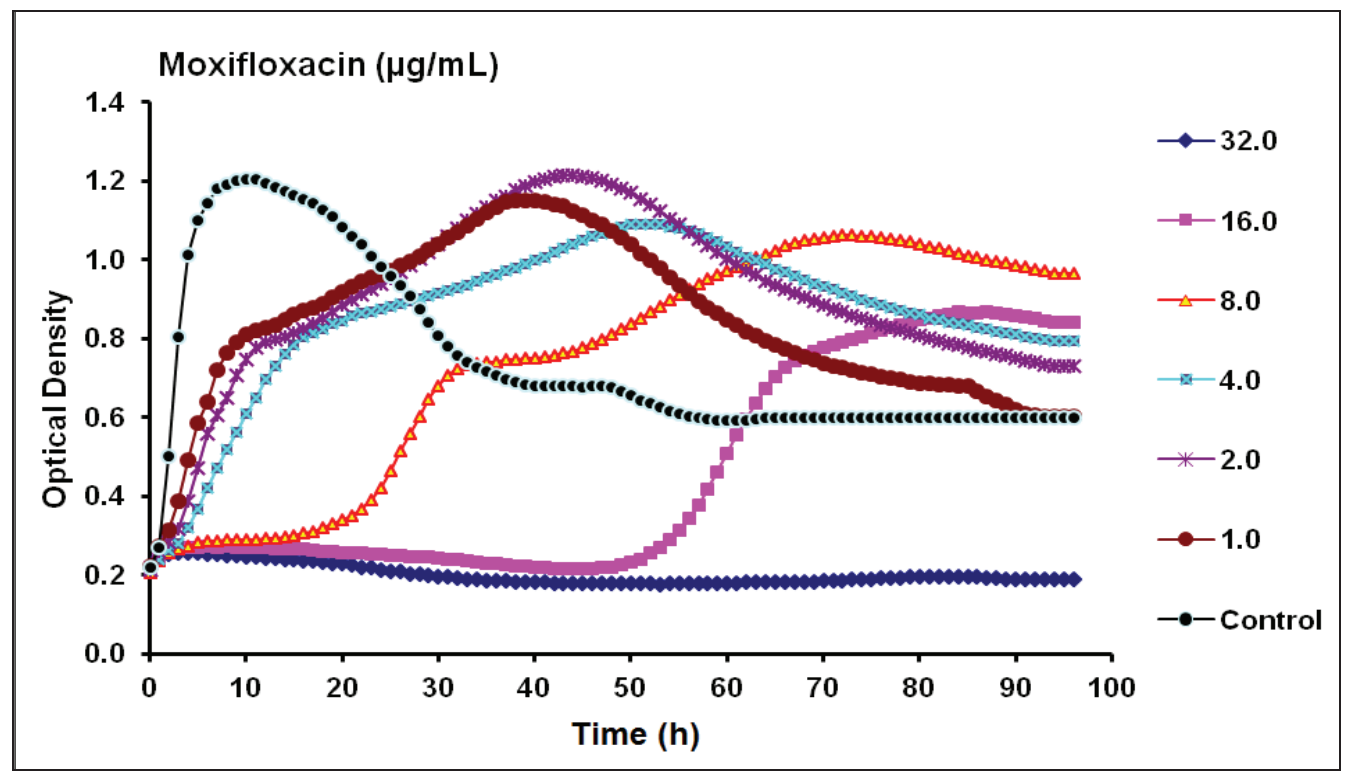

(a)

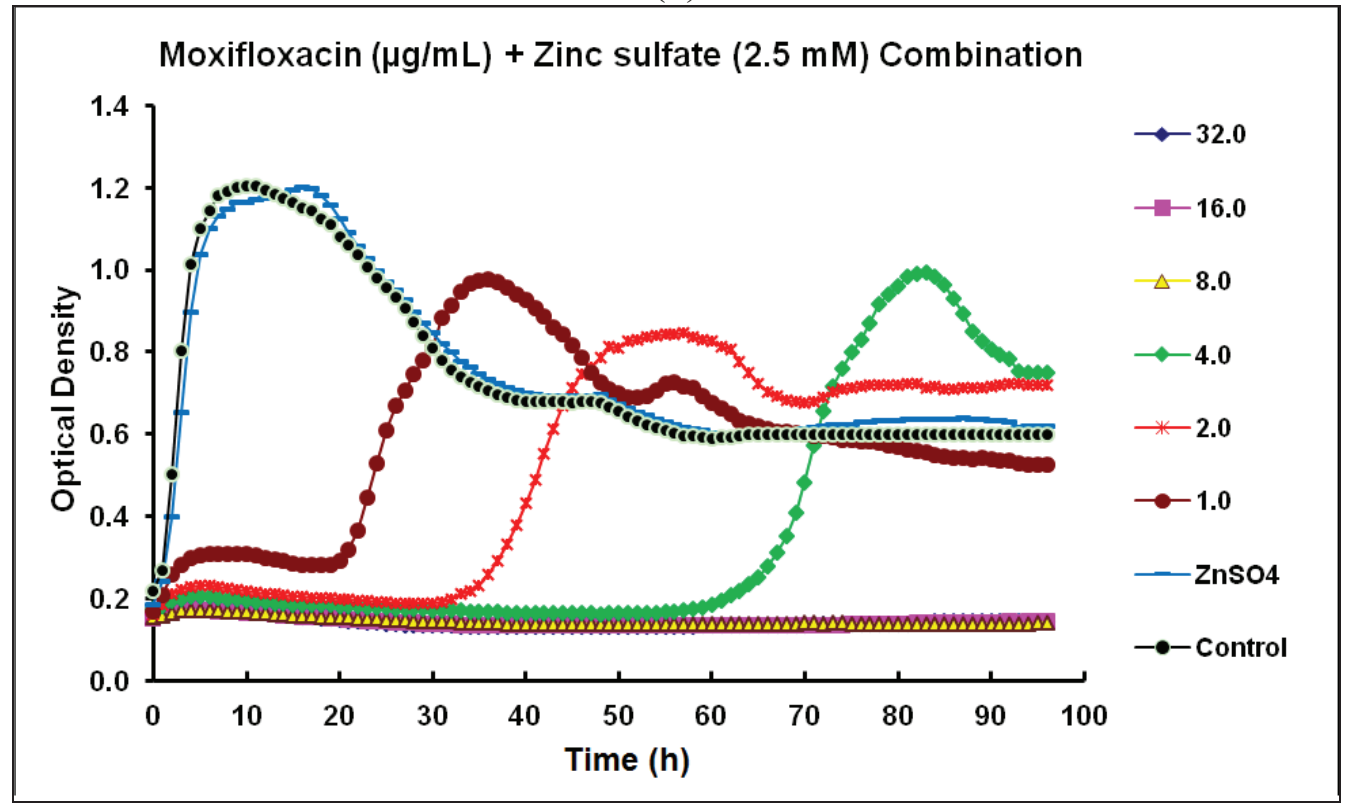

(b)

Regarding norfloxacin, the MIC and MPC against $P$. aeruginosa CCIN34519 biofilm were $5.0 \mu \mathrm{g} / \mathrm{mL}$ and $10.0 \mu \mathrm{g} / \mathrm{mL}$, respectively. Norfloxacin suppressed the biofilm slightly above the baseline and completely at the baseline for 28 and $47 \mathrm{~h}$ at concentration levels of 2.5 and $5.0 \mu \mathrm{g} / \mathrm{mL}$, respectively and its lower concentrations $(0.63-1.25 \mu \mathrm{g} / \mathrm{mL})$ could not suppress the biofilm (Figure $5 \mathrm{a}$ ). On the other hand, the MIC and MPC of norfloxacin, in the presence of zinc sulfate $(2.5 \mathrm{mM})$, diminished to $1.25 \mu \mathrm{g} / \mathrm{mL}$ and $2.5 \mu \mathrm{g} / \mathrm{mL}$, respectively. Furthermore, norfloxacin inhibited the 
biofilm growth for 26 and $96 \mathrm{~h}$ at concentration levels of 1.25 and $2.5 \mu \mathrm{g} / \mathrm{mL}$, respectively (Figure 5b).

Figure 5. Real-time data showing the effect of (a) Norfloxacin alone $(0.63-10.0 \mu \mathrm{g} / \mathrm{mL})$ and (b) Norfloxacin $(0.63-10.0 \mu \mathrm{g} / \mathrm{mL})$ in combination with zinc sulfate $(2.5 \mathrm{mM})$ on $P$. aeruginosa CCIN34519 biofilm as monitored by the Bioscreen C over $96 \mathrm{~h}$. Control represents cation adjusted Mueller Hinton II broth (CAMH) while, $\mathrm{ZnSO}_{4}$ indicates CAMH broth supplemented with $2.5 \mathrm{mM}$ zinc sulfate.

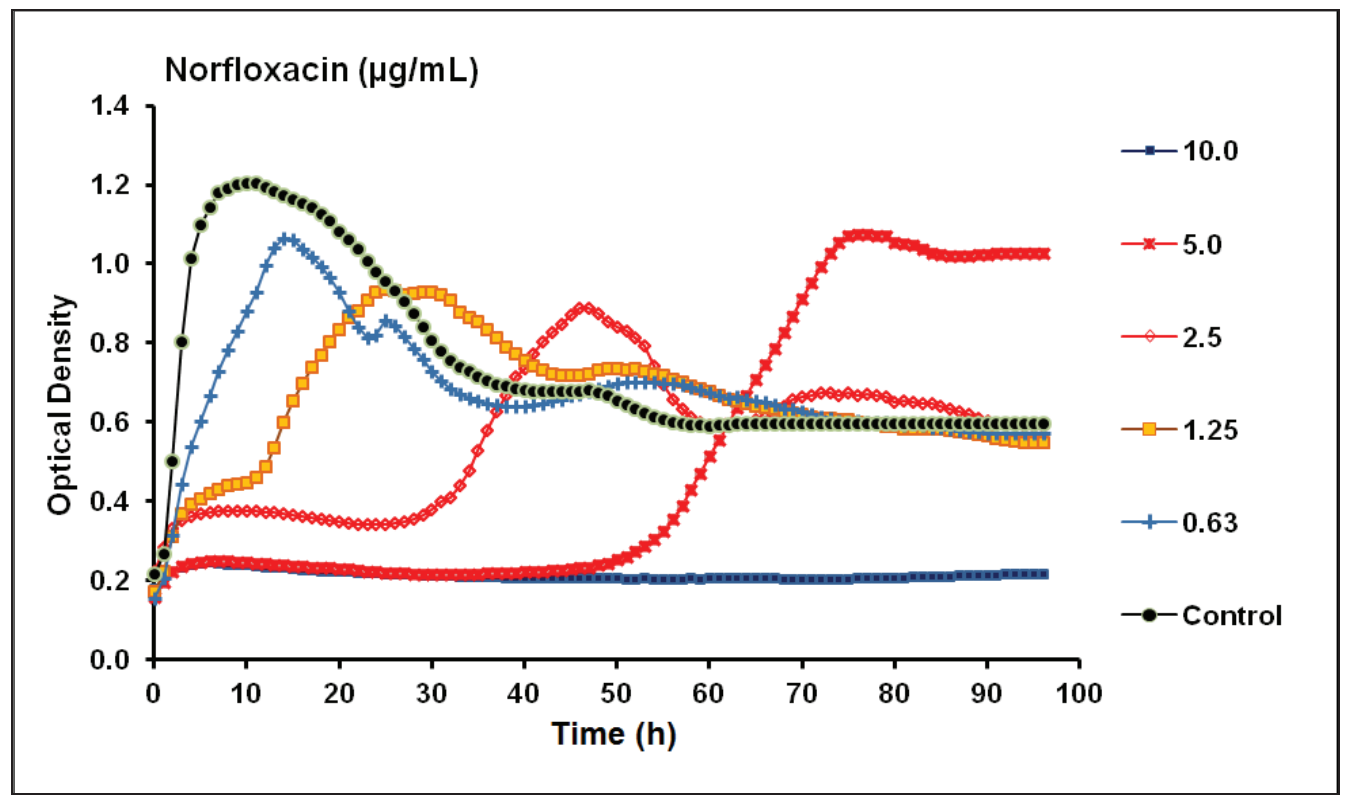

(a)

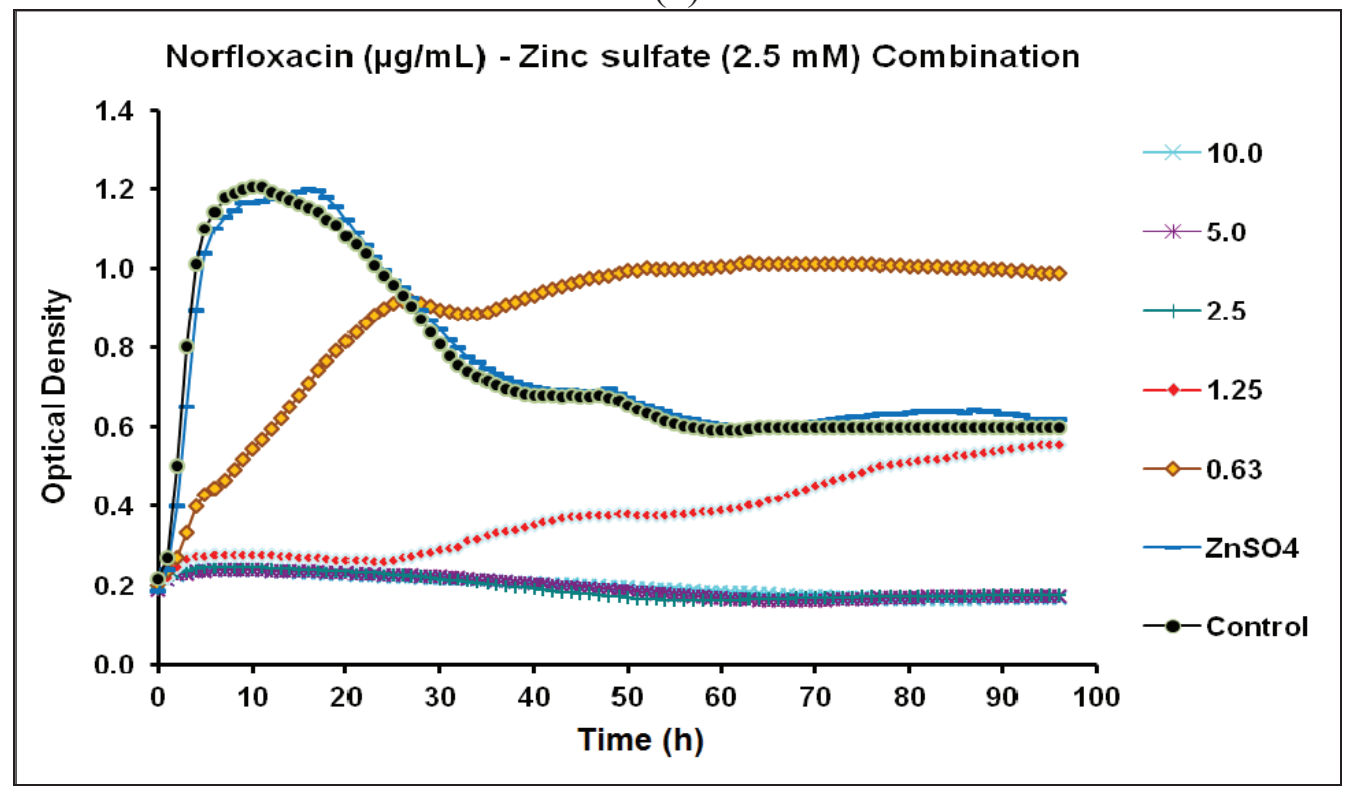

(b)

The MIC and MPC of ertapenem against P. aeruginosa CCIN34519 biofilm maintained the same value of $20 \mu \mathrm{g} / \mathrm{mL}$. Real-time data demonstrated that ertapenem alone suppressed the biofilm growth for 5 and $10 \mathrm{~h}$ at concentration levels of 5 and $10 \mu \mathrm{g} / \mathrm{mL}$, respectively. Moreover, both concentrations markedly abridged the biofilm growth for up to $30 \mathrm{~h}$ and the biofilm maintained a 
prominent low level of microbial growth from $30-96 \mathrm{~h}$ of incubation with ertapenem $(10 \mu \mathrm{g} / \mathrm{mL})$ as compared to the control (Figure 6a). In the presence of zinc sulfate $(2.5 \mathrm{mM})$, both MIC and MPC of ertapenem against $P$. aeruginosa CCIN34519 biofilm reduced to $10 \mu \mathrm{g} / \mathrm{mL}$. Furthermore, ertapenem inhibited the biofilm growth for up to 5 and $96 \mathrm{~h}$ at concentration levels of 5 and $10 \mu \mathrm{g} / \mathrm{mL}$, respectively and the biofilm showed a marked reduction of the microbial growth over $96 \mathrm{~h}$ with ertapenem $(5 \mu \mathrm{g} / \mathrm{mL})$ as compared to the control (Figure $6 \mathrm{~b}$ ).

Figure 6. Real-time data showing the effect of (a) Ertapenem alone (5.0-40.0 $\mu \mathrm{g} / \mathrm{mL})$ and (b) Ertapenem $(5.0-40.0 \mu \mathrm{g} / \mathrm{mL})$ in combination with zinc sulfate $(2.5 \mathrm{mM})$ on $P$. aeruginosa CCIN34519 biofilm as monitored by the Bioscreen C over $96 \mathrm{~h}$. Control represents cation adjusted Mueller Hinton II broth (CAMH) while, $\mathrm{ZnSO}_{4}$ indicates CAMH broth supplemented with $2.5 \mathrm{mM}$ zinc sulfate.

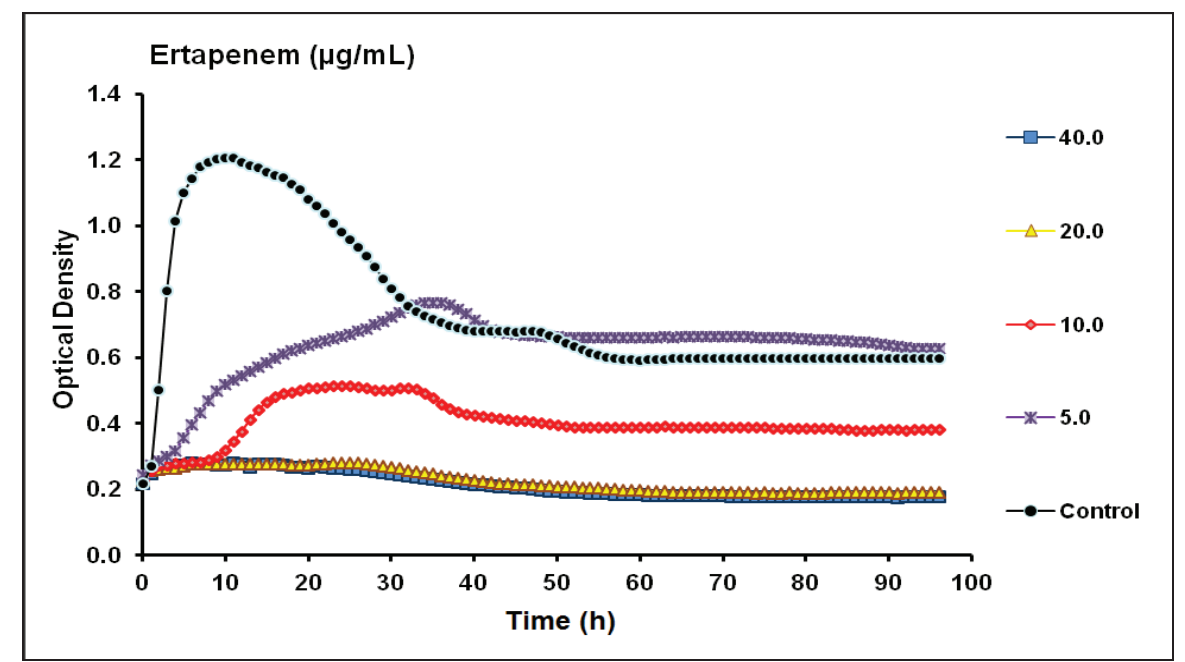

(a)

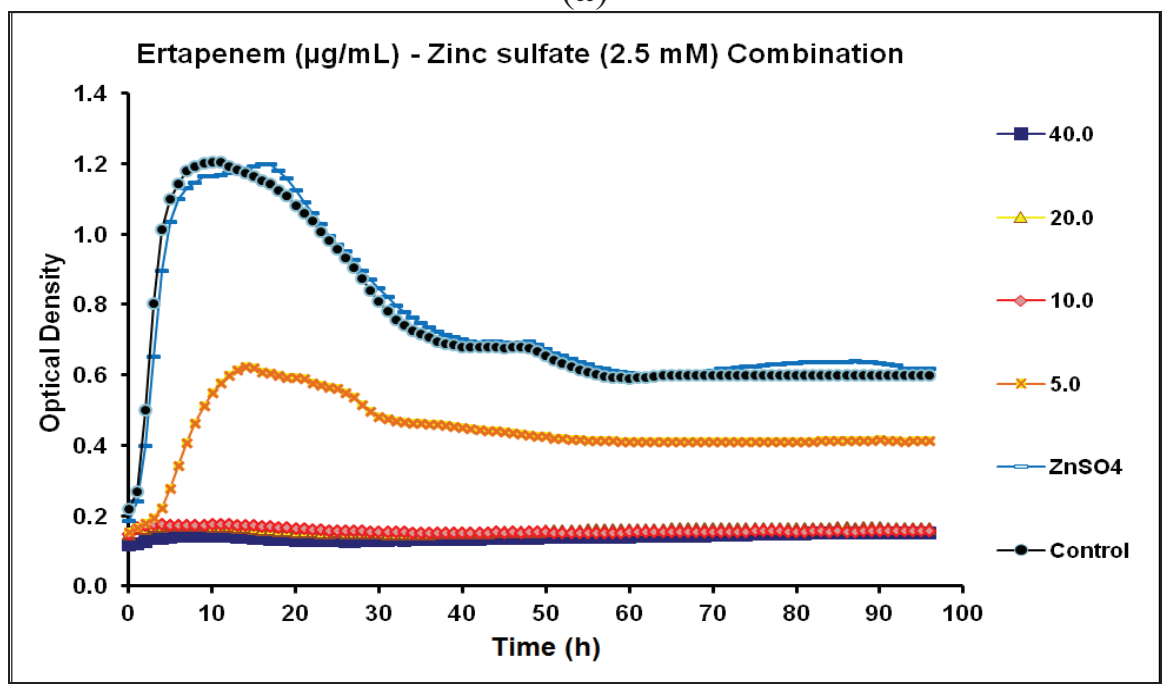

(b)

Concerning ceftriaxone, the MIC and MPC against $P$. aeruginosa CCIN34519 biofilm were $80 \mu \mathrm{g} / \mathrm{mL}$ and $640 \mu \mathrm{g} / \mathrm{mL}$, respectively. Depending on the kinetic data, ceftriaxone alone inhibited the biofilm growth for 20, 28, 48, and $57 \mathrm{~h}$ with the tested concentration levels of 40, 80, 160, and $320 \mu \mathrm{g} / \mathrm{mL}$, respectively (Figure 7a). Conversely, the MIC and MPC of ceftriaxone, in presence of 
zinc sulfate $(2.5 \mathrm{mM})$, considerably increased to $320 \mu \mathrm{g} / \mathrm{mL}$ and $>640 \mu \mathrm{g} / \mathrm{mL}$, respectively. Additionally, ceftriaxone-zinc sulfate $(2.5 \mathrm{mM})$ combination could inhibit the biofilm growth for up to $5,8,16,40$, and $72 \mathrm{~h}$ at concentration levels of $40,80,160,320$, and $640 \mu \mathrm{g} / \mathrm{mL}$, respectively, but none of the tested ceftriaxone concentrations could suppress $P$. aeruginosa CCIN34519 biofilm over 96 h (Figure 7b).

Figure 7. Real-time data showing the effect of (a) Ceftriaxone alone (40-640 $\mu \mathrm{g} / \mathrm{mL})$ and (b) Ceftriaxone $(40-640 \mu \mathrm{g} / \mathrm{mL})$ in combination with zinc sulfate $(2.5 \mathrm{mM})$ on $P$. aeruginosa CCIN34519 biofilm as monitored by the Bioscreen C over $96 \mathrm{~h}$. Control represents cation adjusted Mueller Hinton II broth (CAMH) while, $\mathrm{ZnSO}_{4}$ indicates CAMH broth supplemented with $2.5 \mathrm{mM}$ zinc sulfate.

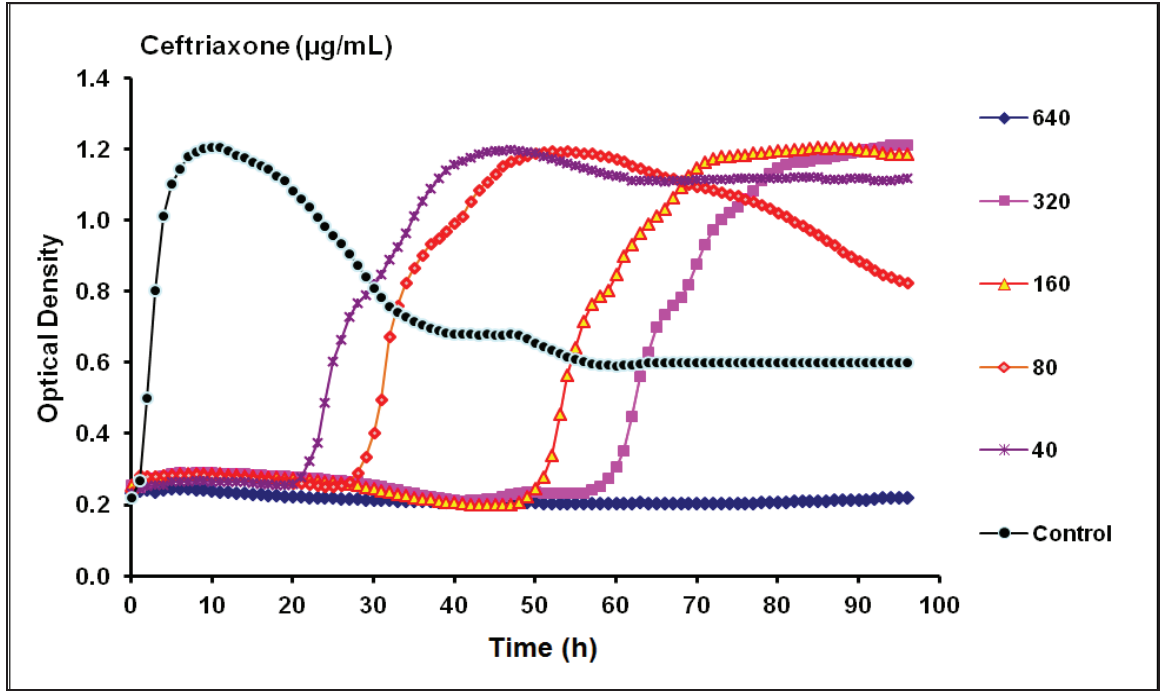

(a)

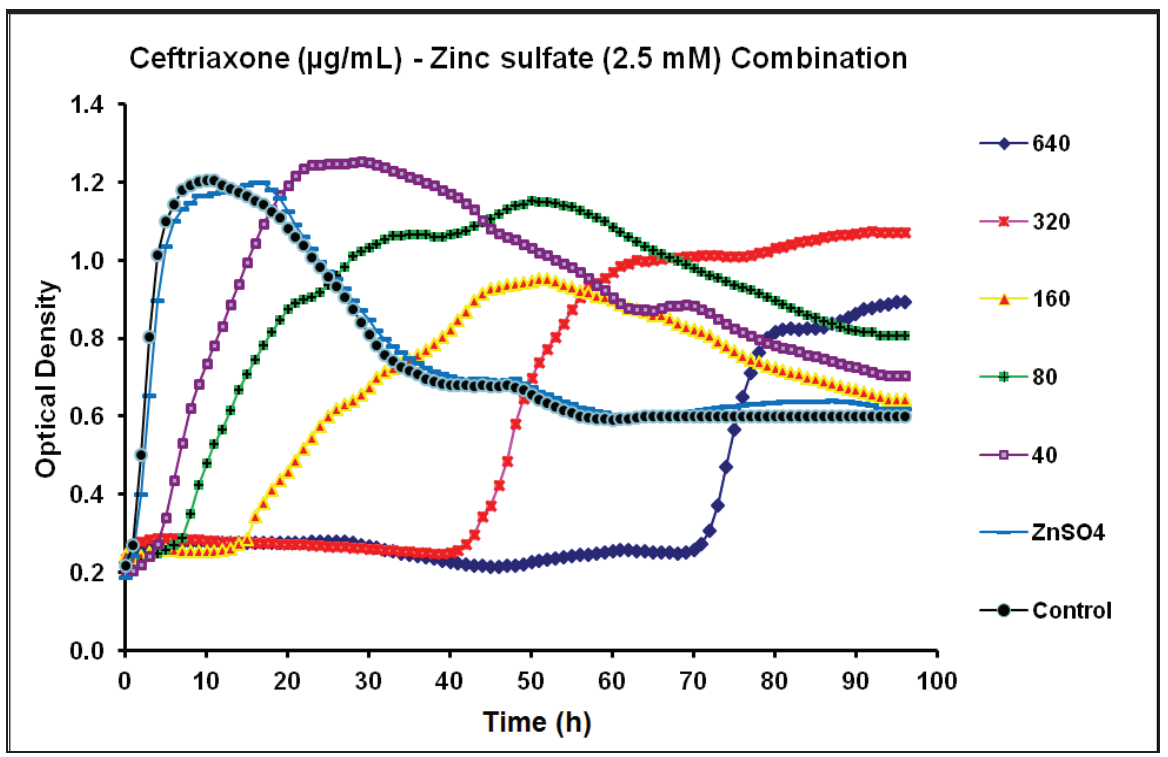

(b)

With gentamicin, the MIC and MPC against $P$. aeruginosa CCIN34519 biofilm maintained the same value of $12.5 \mu \mathrm{g} / \mathrm{mL}$. Gentamicin suppressed the biofilm to some extent above the baseline and entirely at the baseline for 20 and $96 \mathrm{~h}$ at concentration levels of 6.25 and $12.5 \mu \mathrm{g} / \mathrm{mL}$, respectively 
and its lower concentrations $(1.56-3.13 \mu \mathrm{g} / \mathrm{mL}$ ) could not suppress the biofilm (Figure $8 \mathrm{a})$. On the contrary, the MIC and MPC of gentamicin, in presence of zinc sulfate $(2.5 \mathrm{mM})$, substantially increased to $25 \mu \mathrm{g} / \mathrm{mL}$ and $50 \mu \mathrm{g} / \mathrm{mL}$, respectively. Furthermore, gentamicin partially suppressed the biofilm growth, parallel to the baseline at 6.25 and $12.5 \mu \mathrm{g} / \mathrm{mL}$ for 18 and $30 \mathrm{~h}$, respectively, but completely inhibited it at $25 \mu \mathrm{g} / \mathrm{mL}$ for $75 \mathrm{~h}$. Lower concentrations of gentamicin $(1.56-3.13 \mu \mathrm{g} / \mathrm{mL})$ in combination with zinc sulfate $(2.5 \mathrm{mM})$ could not inhibit the biofilm and demonstrated higher growth rate over the initial $10 \mathrm{~h}$ of the incubation period as compared to that of gentamicin alone (Figure 8b).

Figure 8. Real-time data showing the effect of (a) Gentamicin alone $(1.56-50.0 \mu \mathrm{g} / \mathrm{mL})$ and (b) Gentamicin $(1.56-50.0 \mu \mathrm{g} / \mathrm{mL})$ in combination with zinc sulfate $(2.5 \mathrm{mM})$ on P. aeruginosa CCIN34519 biofilm as monitored by the Bioscreen C over $96 \mathrm{~h}$. Control represents cation adjusted Mueller Hinton II broth (CAMH) while, $\mathrm{ZnSO}_{4}$ indicates CAMH broth supplemented with $2.5 \mathrm{mM}$ zinc sulfate.

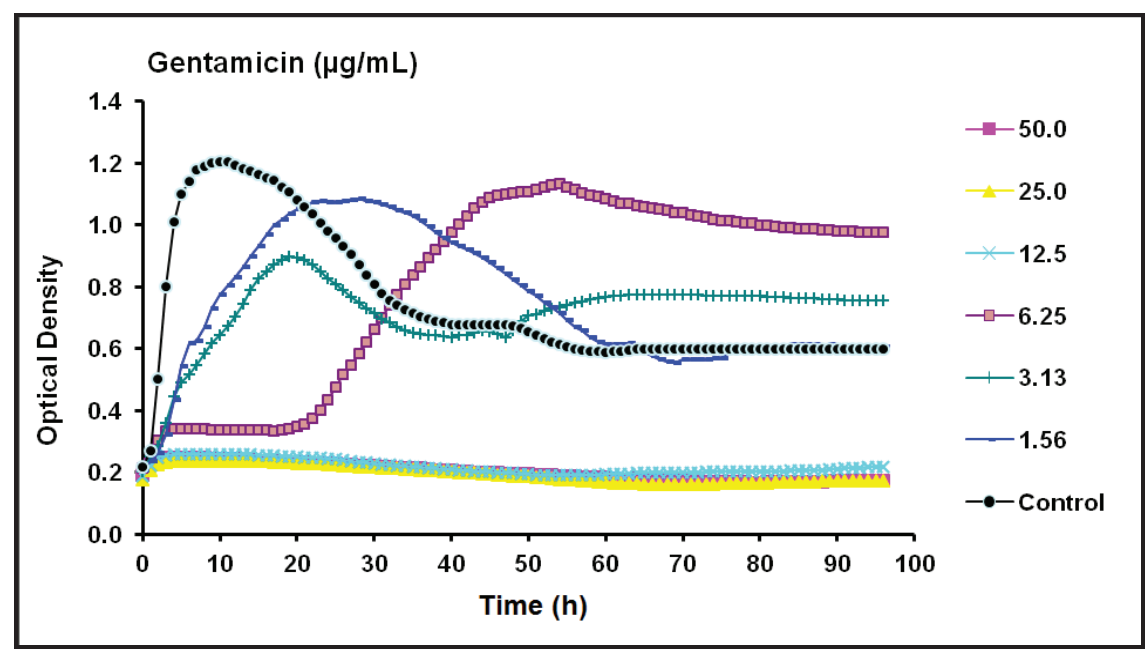

(a)

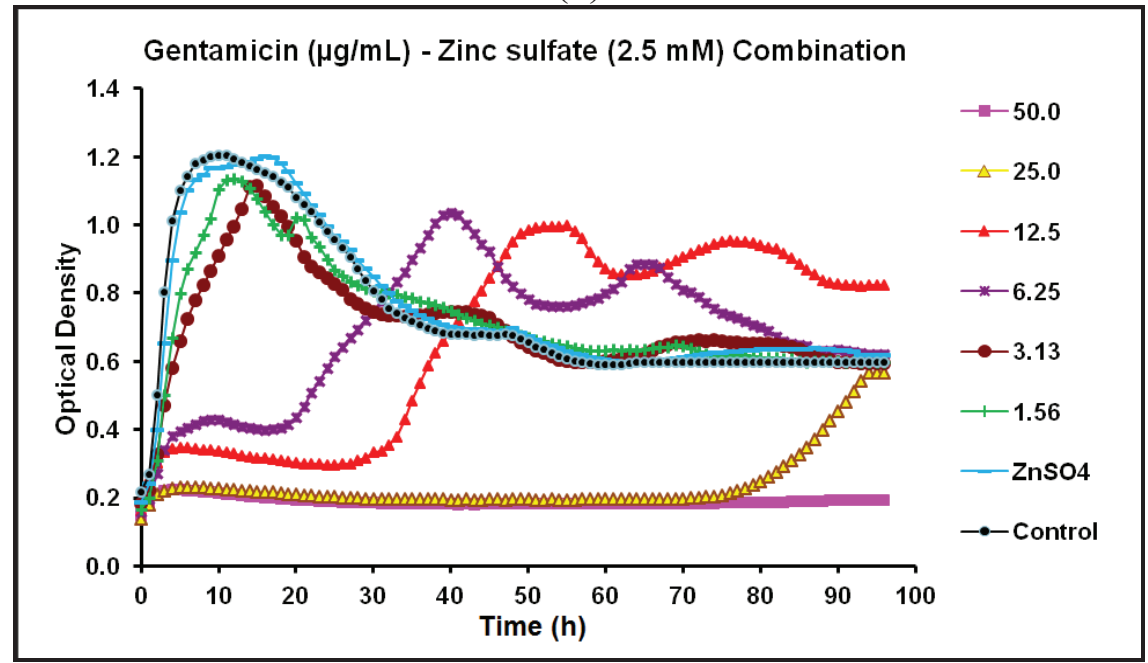

(b)

Regarding tobramycin, the MIC and MPC against $P$. aeruginosa CCIN34519 biofilm were $4.0 \mu \mathrm{g} / \mathrm{mL}$ and $8.0 \mu \mathrm{g} / \mathrm{mL}$, respectively. Tobramycin alone could inhibit the biofilm growth for 22 and $64 \mathrm{~h}$ at concentration levels of 2.0 and $4.0 \mu \mathrm{g} / \mathrm{mL}$, respectively (Figure 9a). Quite the 
opposite, the MIC and MPC of tobramycin, in the presence of zinc sulfate $(2.5 \mathrm{mM})$, noticeably increased to $16 \mu \mathrm{g} / \mathrm{mL}$ and $32 \mu \mathrm{g} / \mathrm{mL}$, respectively. Moreover, tobramycin partially suppressed the biofilm growth at $8.0 \mu \mathrm{g} / \mathrm{mL}$ for $22 \mathrm{~h}$ and completely inhibited it at $16 \mu \mathrm{g} / \mathrm{mL}$ for $76 \mathrm{~h}$. Similar to gentamicin, lower concentrations of tobramycin $(1.0-4.0 \mu \mathrm{g} / \mathrm{mL})$ in combination with zinc sulfate $(2.5 \mathrm{mM})$ could not inhibit the biofilm and showed a higher growth rate over the initial $12 \mathrm{~h}$ of the incubation period as compared to that of tobramycin alone (Figure $9 \mathrm{~b}$ ).

Figure 9. Real-time data showing the effect of (a) Tobramycin alone $(1.0-32.0 \mu \mathrm{g} / \mathrm{mL})$ and (b) Tobramycin $(1.0-32.0 \mu \mathrm{g} / \mathrm{mL})$ in combination with zinc sulfate $(2.5 \mathrm{mM})$ on P. aeruginosa CCIN34519 biofilm as monitored by the Bioscreen C over $96 \mathrm{~h}$. Control represents cation adjusted Mueller Hinton II broth (CAMH) while, $\mathrm{ZnSO}_{4}$ indicates CAMH broth supplemented with $2.5 \mathrm{mM}$ zinc sulfate.

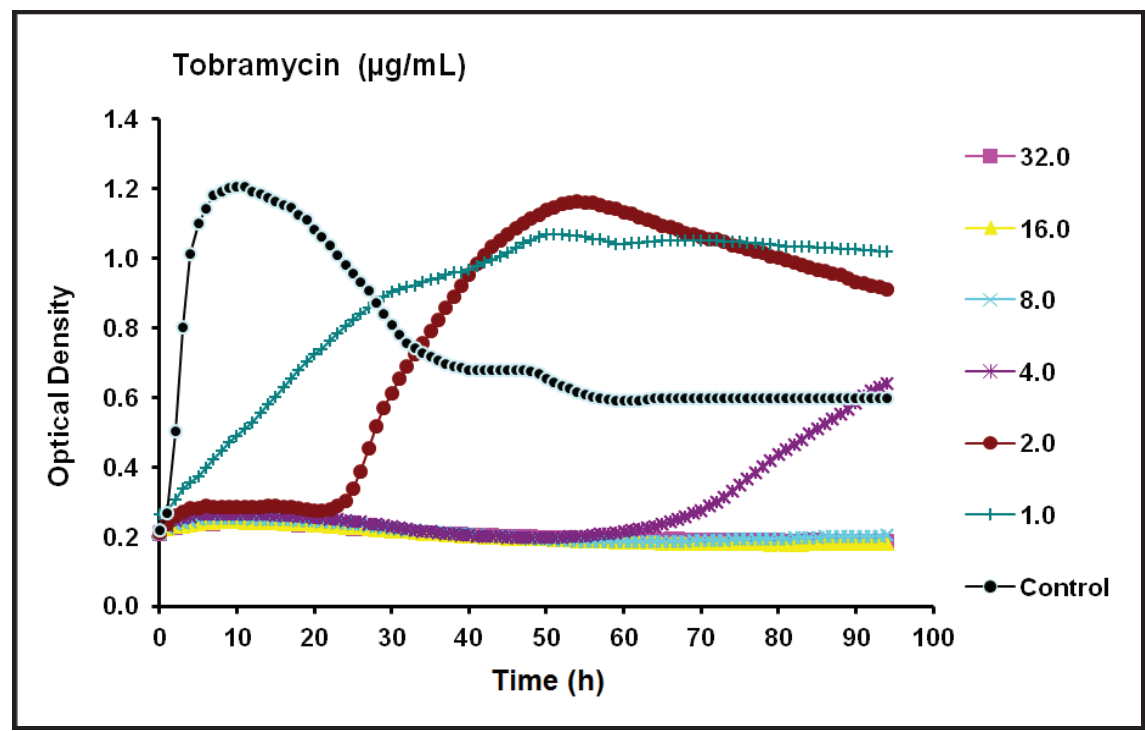

(a)

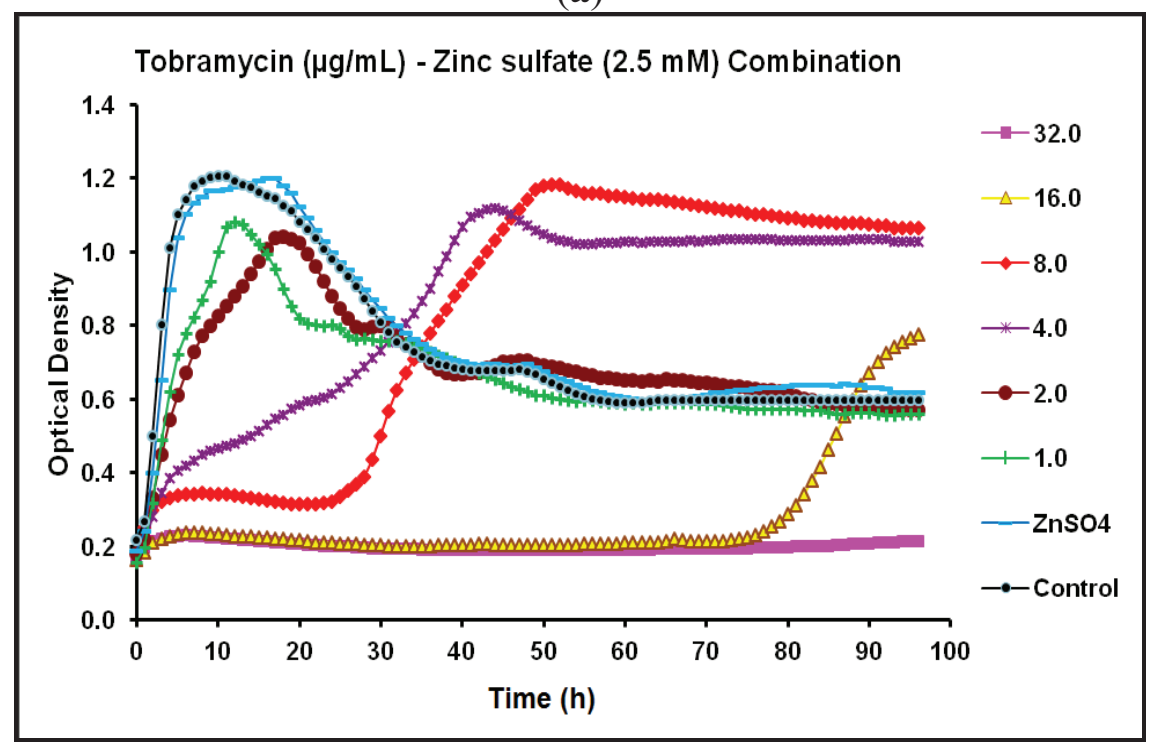

(b)

The synergistic and antagonistic effects of zinc sulfate $(2.5 \mathrm{mM})$ on different tested antibiotics against $P$. aeruginosa CCIN34519 biofilm are summarized in Table 1. 
Table 1. Effects of zinc sulfate on different antibiotics minimum inhibitory concentrations (MICs) and mutant prevention concentrations (MPCs) against P. aeruginosa CCIN34519 biofilm.

\begin{tabular}{|c|c|c|c|c|c|c|}
\hline \multirow{2}{*}{ Antibiotic } & \multirow{2}{*}{ Class } & \multicolumn{2}{|c|}{ Biofilm Without Zinc Sulfate } & \multicolumn{2}{|c|}{ Biofilm With Zinc Sulfate * } & \multirow{2}{*}{$\begin{array}{c}\text { Anti-biofilm Efficacy } \\
\text { of Combination }\end{array}$} \\
\hline & & MIC (24 h) & MPC (96 h) & MIC (24 h) & MPC (96 h) & \\
\hline Ciprofloxacin & Fluoroquinolone & 0.8 & 1.6 & 0.20 & 0.40 & Synergistic \\
\hline Levofloxacin & Fluoroquinolone & 4.0 & 8.0 & 0.25 & 0.50 & Synergistic \\
\hline Moxifloxacin & Fluoroquinolone & 16 & 32 & 2.0 & 8.0 & Synergistic \\
\hline Norfloxacin & Fluoroquinolone & 5.0 & 10 & 1.25 & 2.5 & Synergistic \\
\hline Ertapenem & Carbapenem & 20 & 20 & 10 & 10 & Synergistic \\
\hline Ceftriaxone & Cephalosporin & 80 & 640 & 320 & $>640$ & Antagonistic \\
\hline Gentamicin & Aminoglycoside & 12.5 & 12.5 & 25 & 50 & Antagonistic \\
\hline Tobramycin & Aminoglycoside & 4.0 & 8.0 & 16 & 32 & Antagonistic \\
\hline
\end{tabular}

According to the MICs interpretive standards stated by Clinical and Laboratory Standards Institute (CLSI) [27], P. aeruginosa CCIN34519 biofilm were susceptible to ciprofloxacin and tobramycin; of intermediate resistance to levofloxacin, norfloxacin and gentamicin; and resistant to moxifloxacin, ertapenem and ceftriaxone. Concerning MPC at 96 h, P. aeruginosa CCIN34519 biofilm was considered to demonstrate intermediate resistance to ciprofloxacin, norfloxacin, gentamicin and tobramycin; and be resistant to levofloxacin, moxifloxacin, ertapenem and ceftriaxone. In the presence of zinc sulfate $(2.5 \mathrm{mM}), P$. aeruginosa CCIN34519 biofilm was susceptible to ciprofloxacin, levofloxacin, moxifloxacin and norfloxacin; demonstrate intermediate resistance to ertapenem; and be resistant to ceftriaxone, gentamicin, and tobramycin. On the other hand, at $96 \mathrm{~h}$ and in combination with zinc sulfate $(2.5 \mathrm{mM}), P$. aeruginosa CCIN34519 biofilm maintained its susceptibilities to ciprofloxacin, levofloxacin and norfloxacin; revealed intermediate resistance to moxifloxacin, and ertapenem; and showed resistance to ceftriaxone, gentamicin, and tobramycin.

\subsection{Discussion}

Bacterial biofilms represent global and predominant causes of both chronic infections and indwelling medical devices associated infections such as catheters and prostheses. Such infections typically exhibit significantly enhanced resistance to antimicrobial agents rendering them challenging to treat using conventional chemotherapeutic agents [28]. Standard doses of the antibiotic therapy can reduce the biofilm but rarely eradicates the entire biofilm [29,30]. Some theories attributed the antimicrobial resistance of the biofilm to its physical architecture, mutations, and altered gene expression patterns [31-33]. P. aeruginosa is one of the predominant causes of UTIs, nosocomial and intensive care unit-associated infections that occur annually worldwide [6,34]. As a consequence of biofilm formation, frequency of the resistant P. aeruginosa isolates present in clinical settings has been radically increased, thereby mandating the development of alternative therapeutic strategies [35]. In this context, P. aeruginosa biofilms have demonstrated resistance to different classes of antibiotics including ciprofloxacin (fluoroquinolone), ceftazidime (cephalosporin), and 


\section{6}

tobramycin (aminoglycoside) at concentrations far superior to the therapeutically achievable concentrations [36,37] and one hypothesis explaining biofilm resistance proposes the existence of the persister cells within the biofilm which are metabolically inactive and consequently are not destroyed by antimicrobial agents [38-41].

In the current study, the results of the SEM revealed that $P$. aeruginosa CCIN34519 biofilm could extensively colonize the polystyrene substratum within $12 \mathrm{~h}$ of incubation as well as to develop a relatively uniform architecture. Some investigators reported that a thick $P$. aeruginosa biofilm on surface of the polyethylene tubing was observed by SEM at $48 \mathrm{~h}$ in their in vivo experiments and repeatable pattern of cell death and lysis has been shown in P. aeruginosa biofilm during its development [42]. The difference in time of the biofilm establishment may be attributed to the experimental design and strain difference as well as nature of the used substrata. Main factors influencing the biofilm development may include temperature, nutrient composition, aeration, flow rate, and history of the cultures used for the inoculation, thus the experimental reproducibility can be extensively improved if these factors have been considered [43]. In the present study, the above mentioned variables were efficiently controlled in the conducted experiments through utilizing the Bioscreen $\mathrm{C}$ technology.

It has been demonstrated that $P$. aeruginosa isolates were more resistant to growth inhibition by antibiotics when grown in biofilm cultures than when grown in planktonic ones $[44,45]$. For that reason, the failure of conventional planktonic culture techniques to predict antibiotic susceptibilities may explain inability of the antimicrobial agents to eradicate $P$. aeruginosa biofilm-associated UTIs. In view of that, all the experiments in this study were carried on the biofilm of $P$. aeruginosa CCIN34519 strain recovered from hospitalized patients with urinary tract infection through NAUTICA study [46].

It was previously reported that zinc oxide showed antibacterial and antifungal activity against planktonic cultures of some tested micro-organisms in a concentration dependent manner [47]. Moreover, complexed zinc with protoporphyrin IX or mesoprotoporphyrin IX were both highly effective in negating planktonic growth and biofilm formation of some tested bacteria and these zinc complexes act as iron siderophore analogs by supplanting the natural iron uptake of the tested bacteria [48]. P. aeruginosa regulates multiple genes by the zinc-dependent DksA transcriptional regulator at low zinc conditions. Nevertheless, excess amounts of this trace element can be potentially lethal for the bacteria. Thus, most bacteria have developed systems for both zinc acquisition and detoxification [25]. Concerning the MIC values in this study, zinc sulfate (2.5 $\mathrm{mM})$ could increase the efficacies of ertapenem, ciprofloxacin, levofloxacin, moxifloxacin, and norfloxacin by 2, 4, 16, 8 , and 4 folds, respectively, but decreased the efficacies of ceftriaxone, gentamicin, and tobramycin by 4, 2, and 4 folds, respectively, on $P$. aeruginosa CCIN34519 biofilm. Previous studies mentioned that doses of the antibiotics required to inhibit or eradicate the established biofilms frequently exceed the maximum achievable plasma concentrations for such antibiotics $[44,45]$ and retention of the antibiotics in the anti-pseudomonal cache is a critical issue throughout the world as there is a limited number of effective drugs for treatment of $P$. aeruginosa infections [49]. Generally, fluoroquinolones have a broad spectrum of antimicrobial coverage and ciprofloxacin and, in particular, is one of the relatively effective antibiotics against $P$. aeruginosa infections in clinical settings [50,51]. Although $P$. aeruginosa CCIN34519 biofilm revealed no susceptibility at $96 \mathrm{~h}$ to all the tested 
antibiotics, the biofilm maintained its susceptibility to three fluoroquinolones (ciprofloxacin $[\mathrm{MPC}=0.4 \mu \mathrm{g} / \mathrm{mL}]$, levofloxacin $[\mathrm{MPC}=0.5 \mu \mathrm{g} / \mathrm{mL}$ ], and norfloxacin [MPC $=2.5 \mu \mathrm{g} / \mathrm{mL}]$ ) out of the eight tested antibiotics in the presence of zinc sulfate $(2.5 \mathrm{mM})$ reserving such drugs as effective agents and within the therapeutically achievable concentrations for treatment of $P$. aeruginosa CCIN34519 biofilm in UTIs.

Prior to mutant selection or acquisition of exogenous resistance, $P$. aeruginosa possesses powerful efflux pumps to eliminate almost all classes of antibiotics $[49,52]$ and the concept of its being a super bug was built on such a foundation [13]. The reported MPCs for some fluoroquinolones against $P$. aeruginosa ranged from 2 to $>32.0 \mu \mathrm{g} / \mathrm{mL}$ depending on the study and the bacterial genotype. Nevertheless, the first-step par $C$ gene mutation dramatically increases the MPC far above the original MPC [53-56]. Consistent with these findings, MPCs of the tested fluoroquinolones in this study ranged from 1.6-32.0 $\mu \mathrm{g} / \mathrm{mL}$ against $P$. aeruginosa CCIN34519 biofilm. On the other hand, range of the MPCs for such fluoroquinolones prominently decreased to $0.4-8.0 \mu \mathrm{g} / \mathrm{mL}$ in combination with zinc sulfate $(2.5 \mathrm{mM})$. In presence of zinc sulfate $(2.5 \mathrm{mM})$, real-time data revealed that the tested fluoroquinolones (ciprofloxacin, levofloxacin, moxifloxacin, and norfloxacin) at lower concentrations $(0.2,0.25,4.0$, and $1.25 \mu \mathrm{g} / \mathrm{mL}$, respectively) could suppress the growth of P. aeruginosa CCIN34519 biofilm for longer periods of time (60, 36, 58, and $26 \mathrm{~h}$, respectively) and ertapenem $(5.0 \mu \mathrm{g} / \mathrm{mL})$ noticeably reduced the biofilm growth as compared to such antibiotics alone at the same concentration levels. Consequently, these antibiotics may afford better antimicrobial coverage against $P$. aeruginosa biofilm for extended duration and within the same therapeutic doses in case of concomitant administrations with zinc sulfate.

The potential for serious antagonistic effects by zinc sulfate $(2.5 \mathrm{mM})$ combination with cephalosporin (ceftriaxone) or aminoglycosides (gentamicin and tobramicin) underscores the inevitability to circumvent the concomitant administration of over the counter (OTC) zinc sulfate containing preparations with the prescribed cephalosporins and aminoglycosides during the therapy of $P$. aeruginosa biofilm related infections such as nosocomial catheter-associated UTI as well as soft tissue infections. In the current study, the observed antagonism by zinc sulfate combination with gentamicin and tobramicin against $P$. aeruginosa CCIN34519 biofilm may be attributed to general interference of the divalent cations with the uptake of the aminoglycosides at both the outer and inner membranes in P. aeruginosa [57]. On the other hand, zinc sulfate $(2.5 \mathrm{mM})$ demonstrated synergistic and antagonistic effects in combination with ertapenem and ceftriaxone, respectively. These paradoxical effects with the tested $\beta$-lactam antibiotics may be explained by the variable zinc affinities to different metallo-beta-lactamases [58]. Fortunately, the UTI patients infected with $P$. aeruginosa biofilm may possess an edge in overcoming the infection when ertapenem or fluoroquinolones (ciprofloxacin, levofloxacin, moxifloxacin, and norfloxacin) have been prescribed with simultaneous administration with zinc sulfate-containing preparations and such cases should be deliberately investigated. 


\section{Experimental}

\subsection{Bacterial Strain and Antimicrobial Agents}

P. aeruginosa CCIN34519 strain used in this study was recovered from hospitalized patients with urinary tract infection through North American Urinary Tract Infection Collaborative Alliance (NAUTICA) study [46]. Before each experiment, P. aeruginosa CCIN34519 was sub-cultured on Mueller Hinton agar and incubated for $18-20 \mathrm{~h}$ at $37{ }^{\circ} \mathrm{C}$. The inoculum was then prepared in cation-adjusted Mueller Hinton II broth (CAMH) and diluted to match 0.5 McFarland standard, which is equivalent to $1.5 \times 10^{8} \mathrm{CFU} / \mathrm{mL}$. Ceftriaxone sodium, ciprofloxacin hydrochloride, gentamicin sulfate, levofloxacin, moxifloxacin hydrochloride, norfloxacin, tobramycin, and zinc sulfate heptahydrate were purchased from Sigma-Aldrich (St. Louis, MO, USA). Ertapenem disodium powder was obtained from Thermo-Fisher Scientific (Waltham, MA, USA). All antibiotic stock solutions and dilutions were prepared according to the Clinical and Laboratory Standards Institute (CLSI) guidelines [27]. Stock solutions of the antibiotics were stored at $-80{ }^{\circ} \mathrm{C}$ and aliquots of such solutions were thawed at room temperature and diluted in CAMH broth before each experiment.

\subsection{Scanning Electron Microscopy (SEM)}

For visualization of $P$. aeruginosa CCIN34519 biofilm, overnight broth culture of $P$. aeruginosa CCIN34519 in CAMH broth was diluted in PBS to $1.5 \times 10^{8} \mathrm{CFU} / \mathrm{mL}$ using $0.5 \mathrm{McF}$ arland equivalence turbidity standards (Thermo Scientific RemelTM, Lenexa, KS, USA). The cell suspension was used to inoculate sterile CAMH broth at final bacterial count of $1.5 \times 10^{6} \mathrm{CFU} / \mathrm{mL}$ and $1.5 \mathrm{~mL}$ aliquots of the inoculated broth was distributed in 24-well polystyrene plates (Corning $\operatorname{Costar}{ }^{\circledR}$, Corning, NY, USA). The plates were incubated for $12 \mathrm{~h}$ at $37{ }^{\circ} \mathrm{C}$ without shaking. After incubation, the supernatant was carefully decanted and the wells were washed twice with $2 \mathrm{~mL}$ PBS to remove the unattached or loosely attached bacterial cells. Fixation of $P$. aeruginosa CCIN34519 biofilm was conducted at $4{ }^{\circ} \mathrm{C}$ with $10 \%$ glutaraldehyde (Sigma-Aldrich) for $24 \mathrm{~h}$. Subsequently, the wells were rinsed with a graded series $(30 \%, 50 \%, 70 \%$, and $100 \% \mathrm{v} / \mathrm{v})$ of ethanol (Fisher Scientific, Waltham, MA, USA) for dehydration of biofilm specimens and the wells were then entirely air dried prior to SEM examinations [59]. The photo was captured using variable pressure JEOL scanning electron microscope (Model JSM-6490LV, Peabody, MA, USA) outfitted with a tungsten filament of $10 \mathrm{kV}$ accelerating voltages and chamber variable pressure from $60-70 \mathrm{~Pa}$. The adjustable pressure operation enables lower vacuum to exist at the sample chamber. This feature makes it possible to directly observe frozen or non-conductive specimens. The variable pressure chamber of JSM-6490LV also provides a positively ionized gas immediately above the specimen that in turn dissipates the negative charges that accumulate on the sample surface during scanning and interfere with image acquisition. In view of that, non-conductive biofilm specimens can be imaged without application of a conductive gold coating.

\subsection{Real-Time Monitoring of P. aeruginosa CCIN34519 Biofilm}

A previously described in vitro system for real-time monitoring of biofilm growth/inhibition [60] was utilized with minor adaptations to assess the antimicrobial effects of as single antibiotics as 
well as in combinations with zinc sulfate $(2.5 \mathrm{mM})$ against $P$. aeruginosa CCIN34519 biofilm. Initial $\log$ phase inocula $\left(1 \times 10^{6} \mathrm{CFU} / \mathrm{mL}\right)$ of $P$. aeruginosa CCIN34519 cultures were seeded (150 $\mu \mathrm{L} /$ well) into 100-well polystyrene honeycomb plates (Growth Curves USA, Piscataway, NJ, USA). $P$. aeruginosa CCIN34519 biofilms were allowed to develop in the incubator over $12 \mathrm{~h}$ at $37{ }^{\circ} \mathrm{C}$. After incubation, each well of the honeycomb plates were carefully rinsed twice with $150 \mu \mathrm{L}$ sterile saline for removal of planktonic cells using digital multichannel pipettor adapted for this system. Cation-adjusted Mueller Hinton II (CAMH) broths supplemented with two-fold escalating concentrations of ciprofloxacin, levofloxacin, moxifloxacin, norfloxacin, ertapenem, ceftriaxone, gentamicin, and tobramycin as single antibiotics as well as in combinations with zinc sulfate $(2.5 \mathrm{mM})$ were prepared and transferred to the established P. aeruginosa CCIN34519 biofilm $(250 \mu \mathrm{L} /$ well $)$ in the honeycomb plates. Plates containing treated, untreated $P$. aeruginosa CCIN34519 biofilm, and negative controls were then placed in the preheated incubating chamber of the Bioscreen $\mathrm{C}$ which was programmed to maintain a temperature at $37{ }^{\circ} \mathrm{C}$ without shaking. Growth control wells of $P$. aeruginosa CCIN34519 biofilm treated with CAMH and CAMH spiked with zinc sulfate $(2.5 \mathrm{mM})$ were also implemented in each experiment. Furthermore, negative controls containing CAMH only, CAMH spiked with zinc sulfate $(2.5 \mathrm{mM})$, and CAMH broths supplemented with the above mentioned antibiotics as single agents as well as in combinations with zinc sulfate $(2.5 \mathrm{mM})$ without the biofilm were also involved in the experiments to assure the sterility and stability of their optical density readings overall during $96 \mathrm{~h}$ of the incubation period.

In real-time, $P$. aeruginosa CCIN34519 biofilm growth/inhibition was monitored using the automated technology of Bioscreen C (Growth Curves USA). The Bioscreen C monitors microbial growth through determining the optical densities in each well. Alterations in the optical densities due to the microbial growth or lysis from the biofilm were measured kinetically with an advanced photometrical technology of the Bioscreen C. A wide-band filter with spectrum range of 420-580 nm was used to measure the optical densities in all experiments. This filter has been utilized in the biofilm research because its sensitivity is not affected by the color transformation resulting from the growth of $P$. aeruginosa biofilm [60]. Each biofilm growth curve was repeated three times and the optical density values over $96 \mathrm{~h}$ were robotically recorded at $1 \mathrm{~h}$ intervals using EZExperiment software (Growth Curves USA) and exported to spread sheets for processing and graphical presentation. Minimum inhibitory concentration (MIC) of the biofilm was defined as the antibiotic concentration at which the optical density remains at the baseline $(0.2-0.3)$ at the $24 \mathrm{~h}$ time point, while mutant prevention concentration (MPC) was reported as the antibiotic concentration that prevents growth and development of the resistant subpopulations from the biofilm at $96 \mathrm{~h}$ time point $[60,61]$.

\section{Conclusions}

This study demonstrated a heterogeneous range of anti-biofilm efficacies of the antibiotics in combination with zinc sulfate $(2.5 \mathrm{mM})$ against $P$. aeruginosa CCIN34519 biofilm with potential synergisms in cases of fluoroquinolones (ciprofloxacin, levofloxacin, moxifloxacin, and norfloxacin) and carbapenem (ertapenem) as demonstrated by the reduction of their MICs and MPCs. On the contrary, considerable antagonisms were observed with cephalosporin (ceftriaxone) and aminoglycosides (gentamicin, and tobramycin) as shown by the increment of their MICs and 
MPCs. Further deliberate in vivo investigations for the promising synergisms are required to evaluate their therapeutic potentials for treatment of UTIs caused by P. aeruginosa biofilms.

\section{Acknowledgment}

$P$. aeruginosa CCIN34519 strain used in this study was kindly provided by George Zhanel, Faculty of Medicine, University of Manitoba; Winnipeg, Manitoba, Canada through NAUTICA study.

\section{Author Contributions}

This work was carried out with collaboration between the authors. Walid Elkhatib and Ayman Noreddin conceived the hypothesis and idea of the research project. Ayman Noreddin participated in the study design, data analysis, and literature reviewing. Walid Elkhatib performed the experiments, produced the first draft of the manuscript, and participated in the data analysis. The authors revised and approved the final manuscript for publication.

\section{Abbreviations}

$\begin{array}{ll}\text { CLSI } & \text { Clinical and Laboratory Standards Institute } \\ \text { MIC } & \text { Minimum Inhibitory Concentration } \\ \text { MPC } & \text { Mutant Prevention Concentration } \\ \text { NAUTICA } & \text { North American Urinary Tract Infection Collaborative Alliance } \\ \text { OTC } & \text { Over The Counter } \\ \text { P. aeruginosa } & \text { Pseudomonas aeruginosa } \\ \text { SEM } & \text { Scanning Electron Microscope } \\ \text { UTI } & \text { Urinary Tract Infection }\end{array}$

\section{Conflicts of Interest}

The authors declare no conflict of interest.

\section{References}

1. Parsek, M.R.; Singh, P.K. Bacterial biofilms: An emerging link to disease pathogenesis. Annu. Rev. Microbiol. 2003, 57, 677-701.

2. Drenkard, E. Antimicrobial resistance of Pseudomonas aeruginosa biofilms. Microbes Infect. 2003, 5, 1213-1219.

3. Bjarnsholt, T.; Jensen, P.; Fiandaca, M.J.; Pedersen, J.; Hansen, C.R.; Andersen, C.B.; Pressler, T.; Givskov, M.; Høiby, N. Pseudomonas aeruginosa biofilms in the respiratory tract of cystic fibrosis patients. Pediatr. Pulmonol. 2009, 44, 547-558.

4. Wagner, V.E.; Iglewski, B.H. Pseudomonas aeruginosa biofilms in CF Infection. Clin. Rev. Allergy Immunol. 2008, 35, 124-134.

5. Chang, S.L.; Shortliffe, L.D. Pediatric urinary tract infections. Pediatr. Clin. North. Am. 2006, 53, 379-400. 
6. Mittal, R.; Aggarwal, S.; Sharma, S.; Chhibber, S.; Harjai, K. Urinary tract infections caused by Pseudomonas aeruginosa. J. Infect. Public Health 2009, 2, 101-111.

7. Foxman, B. Epidemiology of urinary tract infections: Incidence, morbidity, and economic costs. Dis. Mon. 2003, 49, 53-70.

8. Litwin, M.S.; Saigal, C.S.; Yano, E.M.; Avila, C.; Geschwind, S.A.; Hanley, J.M. Urologic Diseases in America Project: Analytical methods and principal findings. J. Urol. 2005, 173, 933-937.

9. Ghafoor, A.; Hay, I.; Rehm, B. Role of exopolysaccharides in Pseudomonas aeruginosa biofilm formation and architecture. Appl. Environ. Microbiol. 2011, 77, 5238-5246.

10. Ryder, C.; Byrd, M.; Wozniak, D.J. Role of polysaccharides in Pseudomonas aeruginosa biofilm development. Curr. Opin. Microbiol. 2007, 10, 644-648.

11. Colvin, K.; Gordon, V.; Murakami, K. The Pel polysaccharide can serve a structural and protective role in the biofilm matrix of Pseudomonas aeruginosa. PLoS Pathog. 2011, 7, e1001264.

12. Williamson, K.S.; Richards, L.A.; Perez-Osorio, A.C.; Pitts, B.; McInnerney, K.; Stewart, P.S.; Franklin, M.J. Heterogeneity in Pseudomonas aeruginosa biofilms includes expression of ribosome hibernation factors in the antibiotic-tolerant subpopulation and hypoxia-induced stress response in the metabolically active population. J. Bacteriol. 2012, 194, 2062-2073.

13. Nordmann, P.; Naas, T.; Fortineau, N.; Poirel, L. Superbugs in the coming new decade: Multidrug resistance and prospects for treatment of Staphylococcus aureus, Enterococcus spp. and Pseudomonas aeruginosa in 2010. Curr. Opin. Microbiol. 2007, 1010, 436-440.

14. Algun, U.; Arisoy, A.; Gunduz, T.; Ozbakkaloglu, B. The resistance of Pseudomonas aeruginosa strains to fluoroquinolone group of antibiotics. Indian J. Med. Microbiol. 2004, 22, $112-114$.

15. Henrichfreise, B.; Wiegand, I.; Pfister, W.; Wiedemann, B. Resistance mechanisms of multiresistant Pseudomonas aeruginosa strains from Germany and correlation with hypermutation. Antimicrob. Agents Chemother. 2007, 51, 4062-4070.

16. Huang, S.S.; Lee, S.C.; Lee, N.; See, L.C.; Tsai, M.H.; Shieh, W.B. Comparison of in vitro activities of levofloxacin, ciprofloxacin, ceftazidime, cefepime, imipenem, and piperacillintazobactam against aerobic bacterial pathogens from patients with nosocomial infections. J. Microbiol. Immunol. Infect. 2007, 40, 134-140.

17. Rogues, A.M.; Dumartin, C.; Amadeo, B.; Venier, A.G.; Marty, N.; Parneix, P. Relationship between rates of antimicrobial consumption and the incidence of antimicrobial resistance in Staphylococcus aureus and Pseudomonas aeruginosa isolates from 47 French hospitals. Infect. Control Hosp. Epidemiol. 2007, 28, 1389-1395.

18. Obritsch, M.D.; Fish, D.N.; MacLaren, R.; Jung, R. Nosocomial infections due to multidrug-resistant Pseudomonas aeruginosa: Epidemiology and treatment options. Pharmacotherapy 2005, 25, 1353-1364.

19. Rice, L.B. Unmet medical needs in antibacterial therapy. Biochem. Pharmacol. 2006, 71, 991-995.

20. Wolter, D.J.; Lister, P.D. Mechanisms of $\beta$-lactam resistance among Pseudomonas aeruginosa. Curr. Pharm. Des. 2013, 19, 209-222. 
21. Anderson, G.; Kenney, T.; MacLeod, D. Eradication of Pseudomonas aeruginosa biofilms on cultured airway cells by a fosfomycin/tobramycin antibiotic combination. Pathog. Dis. 2013, $67,39-45$.

22. Tamma, P.; Cosgrove, S.; Maragakis, L. Combination therapy for treatment of infections with gram-negative bacteria. Clin. Microbiol. Rev. 2012, 25, 450-470.

23. Yu, Q.; Griffin, E.; Marquis, S. In vitro evaluation of tobramycin and aztreonam versus Pseudomonas aeruginosa biofilms on cystic fibrosis-derived human airway epithelial cells. J. Antimicrob. Chemother. 2012, 67, 2673-2681.

24. Andreini, C.; Banci, L.; Bertini, I.; Rosato, A. Zinc through the three domains of life. J. Proteome Res. 2006, 5, 3173-3178.

25. Ellison, M.L.; Farrow, J.M., III.; Parrish, W.; Danell, A.S.; Pesci, E.C. The transcriptional Regulator Np20 is the zinc uptake regulator in Pseudomonas aeruginosa. PLoS One 2013, $8, \mathrm{e} 75389$.

26. Sobke, A.; Klinger, M.; Hermann, B.; Sachse, S.; Nietzsche, S.; Makarewicz, O.; Keller, P.M.; Pfister, W.; Straube, E. The urinary antibiotic 5-nitro-8-hydroxyquinoline (Nitroxoline) reduces the formation and induces the dispersal of Pseudomonas aeruginosa biofilms by chelation of iron and zinc. Antimicrob. Agents Chemother. 2012, 56, 6021-6025.

27. Clinical and Laboratory Standards Institute (CLSI). Performance Standards for Antimicrobial Susceptibility Testing. Twenty-First Informational Supplement. CLSI document M100-S21 (ISBN 1-56238-742-1); CLSI: Wayne, PA, USA, 2011.

28. Alkawareek, M.; Algwari, Q.; Laverty, G. Eradication of Pseudomonas aeruginosa biofilms by atmospheric pressure non-thermal plasma. PLoS One 2012, 7, e44289.

29. Costerton, W.; Veeh, R.; Shirtliff, M.; Pasmore, M.; Post, C.; Ehrlich, G. The application of biofilm science to the study and control of chronic bacterial infections. J. Clin. Invest. 2003, $112,1466-1477$.

30. Fux, C.A.; Costerton, J.W.; Stewart, P.S.; Stoodley, P. Survival strategies of infectious biofilms. Trends Microbiol. 2005, 13, 34-40.

31. Mah, T.; Pitts, B.; Pellock, B.; Walker, G.C.; Stewart, P.S.; O’Toole, G.A. A genetic basis for Pseudomonas aeruginosa biofilm antibiotic resistance. Nature 2003, 426, 306-310.

32. Hall-Stoodley, L.; Costerton, J.W.; Stoodley, P. Bacterial biofilms: From the natural environment to infectious diseases. Nat. Rev. Microbiol. 2004, 2, 95-108.

33. Palmer, R.J.; Stoodley, P. Biofilms: Broadened horizons and new emphases. J. Bacteriol. 2007, 189, 7948-7960.

34. Lyczak, J.B.; Cannon, C.; Pier, G.B. Establishment of Pseudomonas aeruginosa infection: Lessons from a versatile opportunist. Microbes Infect. 2000, 2, 1051-1060.

35. Lu, Q.; Yu, J.; Yang, X. Ambroxol interferes with Pseudomonas aeruginosa quorum sensing. Int. J. Antimicrob. Agents 2010, 36, 211-215.

36. Moriarty, T.F.; Elborn, J.S.; Tunney, M.M. Effect of $\mathrm{pH}$ on the antimicrobial susceptibility of planktonic and biofilm-grown clinical Pseudomonas aeruginosa isolates. Br. J. Biomed. Sci. 2007, 64, 101-104. 
37. Walters, M.C.; Roe, F.; Bugnicourt, A.; Franklin, M.J.; Stewart, P.S. Contributions of antibiotic penetration, oxygen limitation, and low metabolic activity to tolerance of Pseudomonas aeruginosa biofilms to ciprofloxacin and tobramycin. Antimicrob. Agents Chemother. 2003, 47, 317-323.

38. Balaban, N.Q.; Merrin, J.; Chait, R.; Kowalik, L.; Leibler, S. Bacterial persistence as a phenotypic switch. Science 2004, 305, 1622-1625.

39. Gefen, O.; Balaban, N.Q. The importance of being persistent: Heterogeneity of bacterial populations under antibiotic stress. FEMS Microbiol. Rev. 2009, 33, 704-717.

40. Lewis, K. Persister cells, dormancy and infectious disease. Nat. Rev. Microbiol. 2007, 5, 48-56.

41. Mulcahy, L.R.; Burns, J.L.; Lory, S.; Lewis, K. Emergence of Pseudomonas aeruginosa strains producing high levels of persister cells in patients with cystic fibrosis. J. Bacteriol. 2010, 192, 6191-6199.

42. Kurosaka, Y.; Ishida, Y.; Yamamura, E.; Takase, H.; Otani, T.; Kumon, H. A non-surgical rat model of foreign body-associated urinary tract infection with Pseudomonas aeruginosa. Microbiol. Immunol. 2001, 45, 9-15.

43. Heydorn, A.; Ersbøll, B.; Kato, J. Statistical analysis of Pseudomonas aeruginosa biofilm development: Impact of mutations in genes involved in twitching motility, cell-to-cell signaling, and stationary-phase sigma factor expression. Appl. Environ. Microbiol. 2002, 68, 2008-2017.

44. Olmos, A.; Castillo, M.; Maiz, L. In vitro prevention of Pseudomonas aeruginosa early biofilm formation with antibiotics used in cystic fibrosis patients. Int. J. Antimicrob. Agent 2012, 40, 173-176.

45. Zhang, L.; Fritsch, M.; Hammond, L. Identification of genes involved in Pseudomonas aeruginosa biofilm-specific resistance to antibiotics. PLoS One 2013, 8, e61625.

46. Zhanel, G.G.; Hisanaga, T.L.; Laing, N.M.; DeCorby, M.R.; Nichol, K.A.; Palatnik, L.P.; Johnson, J.; Noreddin, A.; Harding, G.K.; Nicolle, L.E.; et al. Antibiotic resistance in outpatient urinary isolates: Final results from the North American Urinary Tract Infection Collaborative Alliance (NAUTICA). Int. J. Antimicrob. Agents 2005, 26, 380-388.

47. Pasquet, J.; Chevalier, Y.; Couval, E.; Bouvier, D.; Noizet, G.; Morlière, C.; Bolzinger, M.A. Antimicrobial activity of zinc oxide particles on five micro-organisms of the Challenge Tests related to their physicochemical properties. Int. J. Pharm. 2013, 460, 92-100.

48. Ma, H.; Darmawan, E.T.; Zhang, M.; Zhang, L.; Bryers, J.D. Development of a poly(ether urethane) system for the controlled release of two novel anti-biofilm agents based on gallium or zinc and its efficacy to prevent bacterial biofilm formation. J. Control. Release 2013, 172, 1035-1044.

49. Rossolini, G.M.; Mantengoli, E. Treatment and control of severe infections caused by multiresistant Pseudomonas aeruginosa. Clin. Microbiol. Infect. 2005, 11, 17-32.

50. Adi, H.; Young, P.; Chan, H. Co-spray-dried mannitol-ciprofloxacin dry powder inhaler formulation for cystic fibrosis and chronic obstructive pulmonary disease. Eur. J. Pharm. Sci. 2010, 40, 239-247. 
51. Karlowsky, J.A.; Adam, H.J.; Desjardins, M.; Lagacé-Wiens, P.R.; Hoban, D.J.; Zhanel, G.G. Canadian Antimicrobial Resistance Alliance. Changes in fluoroquinolone resistance over 5 years (CANWARD 2007-11) in bacterial pathogens isolated in Canadian hospitals. J. Antimicrob. Chemother. 2013, 68, 39-46.

52. Bruchmann, S.; Dötsch, A.; Nouri, B.; Chaberny, I.; Häussler, S. Quantitative contributions of target alteration and decreased drug accumulation to Pseudomonas aeruginosa fluoroquinolone resistance. Antimicrob. Agents Chemother. 2013, 57, 1361-1368.

53. Hansen, G.T.; Blondeau, J.M. Comparison of the minimum inhibitory, mutant prevention and minimum bactericidal concentrations of ciprofloxacin, levofloxacin and garenoxacin against enteric Gram-negative urinary tract infection pathogens. J. Chemother. 2005, 17, 484-492.

54. DeRyke, C.A.; Lee, S.Y.; Kuti, J.L.; Nicolau, D.P. Optimising dosing strategies of antibacterials utilizing pharmacodynamic principles: Impact on the development of resistance. Drugs 2006, 66, 1-14.

55. Hansen, G.T.; Zhao, X.; Drlica, K.; Blondeau, J.M. Mutant prevention concentration for ciprofloxacin and levofloxacin with Pseudomonas aeruginosa. Int. J. Antimicrob. Agents 2006, 27, 120-124.

56. Ruiz, J.P.; Quero, Q.J. Pharmacodynamic and pharmacokinetic evaluation of respiratory fluoroquinolones. Guideline to selection of the most appropriate fluoroquinolone. Rev. Esp. Quimioter. 2012, 25, 245-251.

57. Mao, W.; Warren, M.S.; Lee, A.; Mistry, A.; Lomovskaya, O. MexXY-OprM efflux pump is required for antagonism of aminoglycosides by divalent cations in Pseudomonas aeruginosa. Antimicrob. Agents Chemother. 2001, 45, 2001-2007.

58. Murphy, T.A.; Catto, L.E.; Halford, S.E.; Hadfield, A.T.; Minor, W.; Walsh, T.R.; Spencer, J. Crystal structure of Pseudomonas aeruginosa SPM-1 provides insights into variable zinc affinity of metallo-beta-lactamases. J. Mol. Biol. 2006, 357, 890-903.

59. Wen, J.; Zhao, K.; Gu, T.; Raad, I. A green biocide enhancer for the treatment of sulfate-reducing bacteria (SRB) biofilms on carbon steel surfaces using glutaraldehyde. Int. Biodeterior. Biodegrad. 2009, 63, 1102-1106.

60. Elkhatib, W.F.; Haynes, V.L.; Noreddin, A.M. Microbiological appraisal of levofloxacin activity against Pseudomonas aeruginosa biofilm in combination with different calcium channel blockers in vitro. J. Chemother. 2009, 21, 135-143.

61. Smith, H.J.; Nichol, K.A.; Hoban, D.J.; Zhanel, G.G. Stretching the mutant prevention concentration (MPC) beyond its limits. J. Antimicrob. Chemother. 2003, 51, 1323-1325. 


\title{
Chapter 11
}

\section{Role of Old Antibiotics in the Era of Antibiotic Resistance. Highlighted Nitrofurantoin for the Treatment of Lower Urinary Tract Infections}

\section{Maria Jose Munoz-Davila}

\begin{abstract}
Bacterial infections caused by antibiotic-resistant isolates have become a major health problem in recent years, since they are very difficult to treat, leading to an increase in morbidity and mortality. Nitrofurantoin is a broad-spectrum bactericidal antibiotic that, through a complex mode of action which is not completely understood, affects both Gram-negative and Gram-positive bacteria. Nitrofurantoin has been used successfully for a long time for the prophylaxis and treatment of acute lower urinary tract infections in adults, children and pregnant women, but the increased emergence of antibiotic resistance has made nitrofurantoin a suitable candidate for the treatment of infections caused by multidrug-resistant pathogens. Here, we review the mechanism of action, antimicrobial spectrum, pharmacology and safety profile of nitrofurantoin. We also investigate the therapeutic use of nitrofurantoin, including recent data which highlight its role in the management of community urinary tract infection, especially in cases of multidrug-resistant isolates, in which oral active antimicrobials are limited resources nowadays.
\end{abstract}

Reprinted from Antibiotics. Cite as: Munoz-Davila, M.J. Role of Old Antibiotics in the Era of Antibiotic Resistance. Highlighted Nitrofurantoin for the Treatment of Lower Urinary Tract Infections. Antibiotics 2014, 3, 39-48.

\section{Introduction}

Nitrofurantoin, a chemotherapeutic compound of the nitrofuran family, was introduced into clinical practice in 1952. Nitrofurantoin is a synthetic antimicrobial derived from furan by the addition of a nitro group and a side chain containing hydantoin (Figure 1). Nitrofurantoin is a weak acid and its solubility is affected by $\mathrm{pH}$ [1].

Figure 1. Chemical structure of nitrofurantoin.<smiles>O=C1NCC(=O)C(=O)CN1</smiles> 


\section{Mechanism of Action}

The exact mode of action of nitrofurantoin is not completely understood, though it is mainly known to inhibit a number of bacterial enzymes that participate in bacterial carbohydrate metabolism at three points in the Krebs cycle [1] as well as interfering with cell wall synthesis [2]. The nitrogroup coupled onto the heterocyclic furan ring represents the specific active site of the drug and has to be activated by microbial nitroreductases [3].

\section{Antimicrobial Spectrum}

Nitrofurantoin spectrum of in vitro susceptibility includes the majority of Escherichia coli, Citrobacter species, group B streptococci, enterococci, Staphylococcus aureus, S. epidermidis, Klebsiella pneumoniae and Enterobacter species [1]. Thus, its antibacterial spectrum is broad and is particularly effective against the main uropathogens, hence its use for the treatment of urinary tract infections (UTI). Resistance to this drug has remained virtually unchanged since its discovery [4].

\section{Pharmacology}

Nowadays, in Spain, the compound is marketed as both an oral suspension and tablets. The bioavailability is about $90 \%$ and the urinary excretion is $40 \%$ [5].

\subsection{Absorption}

Nitrofurantoin is well absorbed from the gastrointestinal tract with the most absorption occurring in the proximal small bowel. There are many factors which affect bioavailability, but studies have shown that the amount of drug absorbed and the duration of therapeutic urinary concentrations are substantially increased if nitrofurantoin is taken with food [2]. Particle size also affects bioavailability. Thus, the macrocristalline form is more slowly absorbed in the GI tract and is excreted more slowly in the urine than the microcrystalline formulation. This decreased rate of absorption significantly decreases the incidence of nausea and vomiting associated with the microcrystalline form [1]. Due to the effective gastro-intestinal absorption of nitrofurantoin, the effect on the intestinal flora is minimal [6].

\subsection{Distribution and Excretion}

Nitrofurantoin is excreted almost exclusively in the urine and bile. Urinary excretion results from glomerular filtration, tubular secretion and tubular reabsorption. Tubular reabsorption of nitrofurantoin is $\mathrm{pH}$ dependent. Thus, manipulating the urinary $\mathrm{pH}$ permits preferential concentration of nitrofurantoin into the upper or lower urinary tract [7]. Nitrofurantoin does not penetrate aqueous humor, cerebrospinal fluid, prostatic secretions, amniotic fluid or umbilical cord serum in therapeutic concentrations when administered parenterally to man or animals [1]. Oral administration to breast feeding mothers results in minimal drug concentrations in breast milk $[8,9]$. 


\subsection{Excretion in Patients with Impaired Renal Function}

The excretion of the drug is directly related to creatinine clearance [10]. In the presence of impaired renal function, the urine levels fall below the therapeutic range while the serum levels increase into the toxic range. Thus, its efficacy is limited in the setting of renal impairment, with an associated greater risk of toxic effects and adverse reactions [11].

\subsection{Interactions}

It has been suggested that antacid therapy would increase the ionization of nitrofurantoin, resulting in decreased absorption. Also, nitrofurantoin is a potent inhibitor of primary adenine diphosphate-induced platelet aggregation in vitro. Nitrofurantoin can alter a number of laboratory test results. Thus, there may be elevation of the urinary creatinine value. Also, the urine glucose determination using Benedict's qualitative reagent may yield a false-positive result. Serum levels of glucose, bilirubin, alkaline phosphatase and blood urea nitrogen may be spuriously elevated. Also, patients should be warned that the color of the urine may be altered (brown) [1].

\section{Safety Profile}

Nitrofurantoin is, overall, a relatively safe drug. The overall experience after more than three decades of extensive use shows a very low reported side-effect incidence of less than 0.001 per cent based on total courses of therapy [12-14]. However, adverse reactions described, mainly related to long-term usage, have included gastrointestinal disturbances, skin eruptions, hematologic disorders, neurological defects, hepatotoxicity, pulmonary complications and miscellaneous abnormalities. A brief summary of the main adverse reactions is described next.

Gastrointestinal disturbances (anorexia, nausea, vomiting) are the most common side effects. They usually develop during the first week of therapy and efforts have been made to reduce their frequency by altering the nitrofurantoin crystal size, thus modifying the absorption. Skin eruption, consisting of macular, maculopapular or urticarial lesions, are the second most common side effect of nitrofurantoin. Hemolytic anemia in patients with red blood cells deficient in the enzyme glucose-6-phosphate dehydrogenase (G6PD) is a well-documented hematologic complication of nitrofurantoin therapy [2]. A serious adverse reaction to nitrofurantoin described is pheripheral neuropathy [15]. Nitrofurantoin-induced hepatotoxicity is a rare event that usually is readily reversible with discontinuation of the medication. However, nitrofurantoin-induced pulmonary reactions have developed in hundreds of patients. This untoward reaction has been classified arbitrarily into acute, subacute, and chronic forms. The classical acute pulmonary reaction syndrome is characterized by the sudden onset of fever, chills, cough, myalgia and dyspnea. This reaction develops within hours to weeks of the ingestion of the drug. Subacute pulmonary reactions from nitrofurantoin usually develop after one month of drug exposure and are characterized by persistent and progressive cough, dyspnea, orthopnea and fever. The chronic nitrofurantoin pulmonary reaction is associated with the insidious development of non-productive cough and dyspnea. Current guidelines and primary care prescribing systems should emphasize the potential for pulmonary toxicity, which is reversible in case of early recognition [16]. 
The incidence of these side effects is difficult to ascertain, and they are probably on the same order of magnitude (or less) than severe antibiotic associated diarrhoea caused by beta-lactam drugs or fluoroquinolones, or severe skin eruptions caused by trimethoprim/sulfamethoxazole [15].

While medical literature generally defines nitrofurantoin as an antibiotic that is safe for use during the first trimester of pregnancy, new concerns about a possible association between congenital malformations following exposure to nitrofurantoin during the first trimester of pregnancy have recently surfaced. During the last decade, several studies have suggested an increased risk of enophthalmia, cardiovascular malformations, oral cleft, and scull anomalies [17,18]. However, far more studies have suggested that nitrofurantoin is not associated with increased teratogenic risk [19-24]. Also, Goldberg et al. [25] support its use during the first trimester of pregnancy to treat UTI by failing to detect teratogenic risks in a large cohort of exposed women. As far as we know, nitrofurantoin does not cross the placenta [26]. Moreover, overall, it does not appear to exert any untoward effects on the fetus when administered to the pregnant female, except in the latter stages [27]. However, for most outpatient procedures, beta-lactam antibiotics are preferred for the treatment of urinary tract infections in pregnant women.

\section{Therapeutic Use}

\subsection{Classical}

The conventional dosage of nitrofurantoin for an established urinary tract infection is $50 \mathrm{mg}$ or $100 \mathrm{mg}$ four times a day and it started being prescribed both in adults and children for the treatment of acute symptomatic urinary tract infections, also for the treatment of recurrent urinary tract infections and finally for the prophylaxis of recurrent urinary tract infections [1]. Due to its inability to achieve therapeutic blood concentrations, this compound has been relegated to a position of secondary importance. Thus, nitrofurantoin should never be administered to patients with acute bacterial pyelonephritis (as this disease can be accompanied by bacteremia) or to men with recurrent urinary tract infections, as these infections are related with prostatitis and nitrofurantoin does not penetrate tissues well [2].

Another of its main indications is that of bacteriuria of pregnancy, which occurs in approximately 7 to 10 per cent of all pregnant women [28]. Nitrofurantoin is also recommended for the treatment of catheter-associated bacteriuria, which is the most common cause of urinary tract infection. In this sense, nitrofurantoin is used prophylactically during or following urinary tract instrumentation. Nitrofurantoin has been found to prevent bacteriuria in patients with neurogenic bladders using self-catheterization and after intermittent catheterization in patients in the areflexic bladder phase after spinal cord injury [29].

Duration of nitrofurantoin therapy has always remained controversial. Lumbiganon and co-workers sought to find out if the dosing schedule of nitrofurantoin could be decreased from the traditional seven days of treatment to one day to increase compliance, while retaining its efficacy in the treatment of asymptomatic bacteriuria in pregnant women [30]. Although there were no significant differences in symptomatic infections, preterm deliveries and tolerance of subjects were observed between the short and long dosing schedules. More treatment failures, however, were seen in the short-dosing schedule, suggesting the superiority of the traditional-dosing schedule [31]. 
Also, a decrease in the number of doses per day has always been desirable. Recent data indicate that nitrofurantoin three times per day, instead of four, either in adults or in children, could be effective in the management of UTIs, increasing adherence to nitrofurantoin treatment and sparing the use of other antibiotics [32].

Recent data suggest nitrofurantoin as the first drug of choice for treating uncomplicated UTI in women. Nitrofurantoin has been recently compared to trimethoprim/sulfamethoxazole and proved equally effective. Moreover, it was less likely to cause a rash while having similar rates for any adverse event. Based on these findings nitrofurantoin should probably be considered the first drug of choice for treating uncomplicated UTI in women [33]. Also, Gupta et al. [34] reported that a $100 \mathrm{mg}$ bid dosage of nitrofurantoin macrocrystal for 5 days had as good results as classical cotrimoxazole for 3 days for the treatment of acute uncomplicated cystitis in women. Regarding the prevention of recurrent UTI during pregnancy, no significant differences have been found between a combination of suppressive therapy with a daily dose of nitrofurantoin and close surveillance and close surveillance alone. Only sub-analyses in women with more than $90 \%$ follow-up show a decreased incidence of asymptomatic bacteriuria in women who received nitrofurantoin and close surveillance compared with close surveillance only [35]. Finally, one study of long-term antibiotics for preventing recurrent urinary tract infection in children demonstrated that, although nitrofurantoin was more effective than trimethoprim or cotrimoxazole in preventing repeat symptomatic infection or repeat positive urine culture, it was associated with a greater number of side effects, especially in children less than one month of age. The harmful effects of nitrofurantoin outweigh the prophylactic benefit and suggest that nitrofurantoin may not be an acceptable therapy. Patient compliance would be an important factor to consider in deciding on the use of nitrofurantoin as prophylaxis [36].

\subsection{New Applications}

Nowadays, nitrofurantoin is a synthetic nitrofuran antimicrobial agent that has been used for more than 50 years. It still has a role and continues to be prescribed, particularly in the ambulatory setting for uncomplicated UTIs, especially in its macro-crystalline formulation. In recent years, there has been a new interest in "rediscovering" new applications for older antibiotics due to changes in pathogen distribution and resistance. Regarding nitrofurantoin, it has been suggested for the treatment of UTI caused by multiresistant strains.

In this sense, nitrofurantoin is being used increasingly at present to treat vancomycin-resistant enterococci (VRE) nosocomial urinary tract infections (i.e., catheter-associated bacteria). It is one of the few non-ampicillin derivatives that is active against enterococci. Nitrofurantoin is active against both vancomycin-sensitive enterococci (VSE) and VRE. It is the preferred oral antibiotic for nosocomial VSE or VRE catheter-associated bacteriuria [26].

Also, the progressive increase of extended-spectrum b-lactamase (ESBL) producing enteric bacteria in recent years has called for a re-evaluation of current antibiotic therapy for these infections. The rate of resistance to nitrofurantoin in recent surveys in the USA and Canada was $1.1 \%$ among 1,142 isolates of $E$. coli from outpatient urinary isolates [37]. Very similar results were found in France where $1.8 \%$ of all urinary E. coli isolates were resistant to nitrofurantoin in 2005 [38]. However, among 115 clinical isolates of E. coli ESBL producers, only $71.3 \%$ were 
sensitive to nitrofurantoin [39]. Also, E. coli resistance to nitrofurantoin has been reported to be high in a recent survey in Latin American hospitals [40] and in Italy [41]. In K. pneumoniae, the ESBL producers had significantly diminished susceptibility, as compared with a non-ESBL producer, to nitrofurantoin $(p<0.001)$ [42]. Because responses to nitrofurantoin may be less satisfactory and may require longer courses of therapy, nitrofurantoin is considered to be an alternative, rather than a first-line, therapeutic agent for this clinical syndrome [43]. These results are confirmed by Tasbakan et al. [44] who studied a total of 75 patients with lower urinary tract infection caused by culture-proven ESBL-producing nitrofurantoin-sensitive $E$. coli in the urine $\left(>10^{5} \mathrm{CFU} / \mathrm{mm}^{3}\right)$. Microbiological success was defined as a sterile control urine culture and it was achieved in 51 out of 75 patients $(68 \%)$. Also, Chen et al. conclude that nitrofurantoin may be an alternative in the treatment of ESBL-producing E. coli-related lower UTI [43-45].

Nevertheless, activity against $E$. coli non-ESBL is excellent. In a recent study, the susceptibility for $E$. coli was $99.5 \%$. High susceptibility of E. coli clinical isolates to nitrofurantoin $(2.3 \%$ resistance rate), compared to trimethoprim/sulfamethoxazole $(29 \%)$ or ciprofloxacin $(24.2 \%)$ was recently confirmed [46]. However, nitrofurantoin is less susceptible against Gram-negative pathogens other than E. coli, such as Klebsiella spp. (69.2\%) or Enterobacter spp. (63\%). There is no activity against Proteus spp. or P. aeruginosa [47]. Nowadays, in uncomplicated cystitis, antibiotics exclusively reserved for this indication are preferred, in order to reduce antibiotic pressure in this extremely frequent entity [48]. In uncomplicated UTI, E. coli is the most common pathogen, typically being isolated from approximately $80 \%$ of outpatients with acute uncomplicated cystitis across the various regions of the world [49-51]. In clinical practice, urine culture is usually not performed in the setting of community-acquired, uncomplicated cystitis. Antibiotic therapy is therefore mostly empiric and more or less based upon knowledge of national or international surveillance studies. The local resistance levels of $E$. coli therefore determine the empiric antibiotic treatment. The range of pathogens associated with acute uncomplicated pyelonephritis is similar to that seen in acute uncomplicated cystitis [52]. The most recent surveillance study in Europe investigating uncomplicated cystitis is the Antimicrobial Resistance Epidemiology Survey on Cystitis (ARESC) project [53]. The results of the ARESC study showed that antibiotic substances classically used for the treatment of uncomplicated UTIs, such as cotrimoxazole, fluoroquinolones or aminopenicillins, lose their effectiveness due to increasing resistance. Therefore, ideal substances are those with high susceptibility rates, exclusively used for this indication, such as fosfomycin tromethamine, nitrofurantoin or pivmecillinam.

Finally, Slekoveck et al. [54] state that while long-term, prophylactic nitrofurantoin should be restricted, due to secondary effects, this antibiotic should retain its place in the therapeutic armamentarium for UTIs, especially in the face of rising multidrug-resistant Enterobacteriaceae. In their country of origin, prescribers largely replaced nitrofurantoin with fluoroquinolones due to the frequency of nitrofurantoin adverse reactions related to duration of therapy. This had a tremendous impact on increased fluoroquinolone consumption, which has been related to antimicrobial resistance. 


\section{Conclusions}

Nitrofurantoin has been used for a long time, but the emergence of antibiotic resistance and the decline in newly developed antibiotics has increased interest in the treatment of bacterial UTI with this antibiotic. Pharmacology limitations, such as four doses per day needed, may be soon something of the past. Not only has resistance to nitrofurantoin remained virtually unchanged since its discovery but also its safety profile have made it the antimicrobial of choice in the prophylaxis and treatment of lower UTI in adults for many years. In this new microbiological era characterized by multi-drug resistant pathogens, nitrofurantoin's role is crucial.

\section{Conflicts of Interest}

The author declares no conflict of interest.

\section{References}

1. Cunha, B.A. Nitrofurantoin: An update. Obstet. Gynecol. Surv. 1989, 44, 399-406.

2. Gleckman, R.; Alvarez, S.; Joubert, D.W. Drug therapy reviews: Nitrofurantoin. Am. J. Hosp. Pharm. 1979, 36, 342-351.

3. Koulaouzidis, A.; Bhat, S.; Moschos, J.; Tan, C.; de Ramon, A. Nitrofurantoininduced lungand hepatotoxicity. Ann. Hepatol. 2007, 6, 119-121.

4. Hooton, T.M.; Scholes, D.; Gupta, K.; Stapleton, A.E.; Roberts, P.L.; Stamm, W.E. Amoxicillin-clavulanate $v s$. ciprofloxacin for the treatment of uncomplicated cystitis in women: A randomized trial. JAMA 2005, 293, 949-955.

5. Conklin, J.D. The pharmacokinetics of nitrofurantoin and its related bioavailability. Antibiot. Chemother. 1978, 25, 233-252.

6. Mavromanolakis, E.; Maraki, S.; Samonis, G.; Tselentis, Y.; Cranidis, A. Effect of norfloxacin, trimethoprimsulfamethoxazole and nitrofurantoin on fecal flora of women with recurrent urinary tract infections. J. Chemother. 1997, 9, 203-207.

7. Conklin, J.D. Biopharmaceutics of nitrofurantoin. Pharmacology 1972, 8, 178-181.

8. Hosbach, R.; Foster, R.B. Absence of nitrofurantoin from human milk (letter). JAMA 1967, 202, 1057.

9. Varsano, I.; Fischl, J.; Shochet, S.B. The excretion of orally ingested nitrofurantoin in human milk. J. Pediatr. 1973, 82, 886-887.

10. Sachs, J.; Geer, J.; Noell, P.; Kunin, C.M. Effect of renal function on urinary recovery of orally administered nitrofurantoin. N. Engl. J. Med. 1968, 278, 1032-1035.

11. Oplinger, M.; Andrews, C.O. Nitrofurantoin contraindication in patients with a creatinine clearance below $60 \mathrm{~mL} / \mathrm{min}$ : Looking for the evidence. Ann. Pharmacother. 2013, 47, 106-111.

12. Holmberg, L.; Boman, G.; Bottiger, L.S.; Eriksson, B.; Spross, R.; Wessling, A. Adverse reactions to nitrofurantoin. An analysis of 921 reports. Am. J. Med. 1980, 69, 733-738.

13. Koch-Weser, J.; Sidel, V.W.; Dexter, M.; Parish, C.; Finer, D.C.; Kanarek, P. Adverse reactions to sulfisoxazole, sulfamethoxazole, and nitrofurantoin. Arch. Int. Med. 1971, 128, 399-404. 
14. D’Arcy, P.F. Nitrofurantoin. Drug Intell. Clin. Pharm. 1985, 19, 540-547.

15. Tan, I.L.; Polydefkis, M.J.; Ebenezer, G.J.; Hauer, P.; McArthur, J.C. Peripheral nerve toxic effects of nitrofurantoin. Arch. Neurol. 2012, 69, 265-268.

16. Marshall, A.D.; Dempsey, O.J. Is "nitrofurantoin lung" on the increase? Br. Med. J. 2013, $18, \mathrm{f} 3897$.

17. Crider, K.S.; Cleves, M.A.; Reefhuis, J.; Berry, R.J.; Hobbs, C.A.; Hu, D.J. Antibacterial medication use during pregnancy and risk of birth defects: National birth defects prevention study. Arch. Pediatr. Adolesc. Med. 2009, 163, 978-985.

18. Gardner, J.S.; Guyard-Boileau, B.; Alderman, B.W.; Fernbach, S.K.; Greene, C.; Mangione, E.J. Maternal exposure to prescription and non-prescription pharmaceuticals or drugs of abuse and risk of craniosynostosis. Int. J. Epidemiol. 1998, 27, 64-67.

19. Briggs, G.G.; Freeman, R.K.; Yaffe, S.J. Drugs in Pregnancy and Lactation: A Reference Guide to Fetal and Neonate Risk, 8th ed.; Lippincott Williams \& Wilkins: Philadelphia, PA, USA, 2008; pp. 1310-1312.

20. Prytherch, J.P.; Sutton, M.L.; Denine, E.P. General reproduction, perinatal-postnatal, and teratology studies of nitrofurantoin macrocrystals in rats and rabbits. J. Toxicol. Environ. Health 1984, 13, 811-823.

21. Lee, M.; Bozzo, P.; Einarson, A.; Koren, G. Urinary tract infections in pregnancy. Can. Fam. Physician 2008, 54, 853-854.

22. Hailey, F.J.; Fort, H.; Williams, J.C.; Hammers, B. Foetal safety of nitrofurantoin macrocrystals therapy during pregnancy: A retrospective analysis. J. Int. Med. Res. 1983, 11, 364-369.

23. Czeizel, A.E.; Rockenbauer, M.; Sørensen, H.T.; Olsen, J. Nitrofurantoin and congenital abnormalities. Eur. J. Obstet. Gynecol. Reprod. Biol. 2001, 95, 119-126.

24. Ben David, S.; Einarson, T.; Ben David, Y.; Nulman, I.; Pastuszak, A.; Koren, G. The safety of nitrofurantoinduring the first trimester of pregnancy: Meta-analysis. Fundam. Clin. Pharmacol. 1995, 9, 503-507.

25. Goldberg, O.; Koren, G.; Landau, D.; Lunenfeld, E.; Matok, I.; Levy, A. Exposure to nitrofurantoin during the first trimester of pregnancy and the risk for major malformations. J. Clin. Pharmacol. 2013, 53, 991-995.

26. Cunha, B.A. New uses for older antibiotics: Nitrofurantoin, amikacin, colistin, polymyxin B, doxycycline, and minocycline revisited. Med. Clin. North Am. 2006, 90, 1089-1107.

27. Nardiello, S.; Pizzella, T.; Ariviello, R. Risks of antibacterial agents in pregnancy. Infez. Med. 2002, 10, 8-15.

28. Delzell, J.E., Jr.; Lefevre, M.L. Urinary tract infections during pregnancy. Am. Fam. Physician 2000, 61, 713-721.

29. Cunha, B.A.; Schoch, P.; Hage, J.E. Oral theraphy of catheter-associated bacteriuria (CAB) in the era of antibiotic resistance: Nitrofurantoin revisited. J. Chemother. 2012, 24, 122-124. 
30. Lumbiganon, P.; Villar, J.; Laopaiboon, M.; Widmer, M.; Thinkhamrop, J.; Carroli, G.; Duc, V.N.; Mignini, L.; Festin, M.; Prasertcharoensuk, W.; et al. World Health Organization Asymptomatic Bacteriuria Trial Group. One-day compared with 7-day nitrofurantoin for asymptomatic bacteriuria in pregnancy: A randomized controlled trial. Obstet. Gynecol. 2009, 113, 339-345.

31. Guinto, V.T.; de Guia, B.; Festin, M.R.; Dowswell, T. Different antibiotic regimens for treating asymptomatic bacteriuria in pregnancy. Cochrane Database Syst. Rev. 2010, 8, CD007855.

32. Amábile-Cuevas, C.F.; Arredondo-García, J.L. Antimicrobial activity data in support of nitrofurantoin three times per day. J. Antimicrob. Chemother. 2011, 66, 1652-1653.

33. Zalmanovici Trestioreanu, A.; Green, H.; Paul, M.; Yaphe, J.; Leibovici, L. Antimicrobial agents for treating uncomplicated urinary tract infection in women. Cochrane Database Syst. Rev. 2010, 6, CD007182.

34. Gupta, K.; Hooton, T.M.; Roberts, P.L.; Stamm, W.E. Short-course nitrofurantoin for the treatment of acute uncomplicated cystitis in women. Arch. Int. Med. 2007, 12, 2207-2212.

35. Schneeberger, C.; Geerlings, S.E.; Middleton, P.; Crowther, C.A. Interventions for preventing urinary tract infection during pregnancy. Cochrane Database Syst. Rev. 2012, 14, CD009279.

36. Williams, G.; Craig, J.C. Long-term antibiotics for preventing recurrent urinary tract infection in children. Cochrane Database Syst. Rev. 2011, 16, CD001534.

37. Zhanel, G.G.; Hisanaga, T.L.; Laing, N.M.; DeCorby, M.R.; Nichol, K.A.; Weshnoweski, B.; Johnson, J.; Noreddin, A.; Low, D.E.; Karlowsky, J.A.; et al. Antibiotic resistance in Escherichia coli outpatient urinary isolates: Final results from the North American Urinary Tract Infection Collaborative Alliance (NAUTICA). Int. J. Antimicrob. Agents 2006, 27, 468-475.

38. Honderlick, P.; Cahen, P.; Gravisse, J.; Vignon, D. Quelle sensibilité aux antibiotiques pour les bactéries responsables d'infections urinaires? Que penser de fosfomycine et nitrofuranes? Pathol. Biol. 2006, 54, 462-466.

39. Puerto, A.S.; Fernandez, J.G.; del Castillo, J.; Pino, M.J.; Angulo, J.P. In vitro activity of beta-lactam and non-betalactam antibiotics in extended spectrum beta-lactamase producing clinical isolates of Escherichia coli. Diagn. Microbiol. Infect. Dis. 2006, 54, 135-139.

40. Gales, A.C.; Sader, H.S.; Jones, R.N. SENTRY participants group (Latin America). Urinary tract infection trends in Latin American hospitals: Report from the SENTRY antimicrobial surveillance program (1997-2000). Diagn. Microbiol. Infect. Dis. 2002, 44, 289-299.

41. Fadda, G.; Nicoletti, G.; Schito, G.C.; Tempera, G. Antimicrobial susceptibility patterns of contemporary pathogens from uncomplicated urinary tract infection isolated in a multicenter Italian survey: Possible impact in guidelines. J. Chemother. 2005, 17, 251-257.

42. Procop, G.W.; Tuohy, M.J.; Wilson, D.A.; Williams, D.; Hadziyannis, E.; Hall, G.S. Cross-class resistance to non-beta-lactam antimicrobials in extended-spectrum-beta-lactamaseproducing Klebsiella pneumoniae. Am. J. Clin. Pathol. 2003, 120, 265-267.

43. Garau, J. Other antimicrobials of interest in the era of extended-spectrum beta-lactamases: Fosfomycin, nitrofurantoin and tigecycline. Clin. Microbiol. Infect. 2008, 14, 198-202. 
44. Tasbakan, M.I.; Pullukcu, H.; Sipahi, O.R.; Yamazhan, T.; Ulusoy, S. Nitrofurantoin in the treatment of extended-spectrum $\beta$-lactamase-producing Escherichia coli-related lower urinary tract infection. Int. J. Antimicrob. Agents. 2012, 40, 554-556.

45. Chen, Y.H.; Ko, W.C.; Hsueh, P.R. Emerging resistance problems and future perspectives in pharmacotherapy for complicated urinary tract infections. Expert Opin. Pharmacother. 2013, 14, 587-596.

46. Kashanian, J.; Hakimian, P.; Blute, M., Jr.; Wong, J.; Khanna, H.; Wise, G.; Shabsigh, R. Nitrofurantoin: The return of an old friend in the wake of growing resistance. BJU Int. 2008, 102, 1634-1637.

47. Mazzulli, T.; Skulnick, M.; Small, G.; Marshall, W.; Hoban, D.J.; Zhanel, G.G.; Finn, S.; Low, D.E. Susceptibility of community Gram-negative urinary tract isolates to mecillinam and other oral agents. Can. J. Infect. Dis. 2001, 12, 289-292.

48. Wagenlehner, F.M.; Wullt, B.; Perletti, G. Antimicrobials in urogenital infections. Int. J. Antimicrob. Agents 2011, 38, 3-10.

49. Gupta, K.; Hooton, T.M.; Stamm, W.E. Increasing antimicrobial resistance and the management of uncomplicated community-acquired urinary tract infections. Ann. Int. Med. 2001, 135, 41-50.

50. Kahlmeter, G. An international survey of the antimicrobial susceptibility of pathogens from uncomplicated urinary tract infections: The ECO.SENS project. J. Antimicrob. Chemother. 2003, 51, 69-76.

51. Warren, J.W.; Abrutyn, E.; Hebel, J.R.; Johnson, J.R.; Schaeffer, A.J.; Stamm, W.E. Guidelines for antimicrobial treatment of uncomplicated acute bacterial cystitis and acute pyelonephritis in women, Infectious Diseases Society of America (IDSA). Clin. Infect. Dis. 1999, 29, 745-758.

52. Talan, D.A.; Stamm, W.E.; Hooton, T.M.; Moran, G.J.; Burke, T.; Iravani, A.; Reuning-Scherer, J.; Church D.A. Comparison of ciprofloxacin (7 days) and trimethoprim-sulfamethoxazole (14 days) for acute uncomplicated pyelonephritis pyelonephritis in women: A randomized trial. JAMA 2000, 283, 1583-1590.

53. Naber, K.G.; Schito, G.; Botto, H.; Palou, J.; Mazzei, T. Surveillance study in Europe and Brazil on clinical aspects and antimicrobial resistance epidemiology in females with cystitis (ARESC): Implications for empiric therapy. Eur. Urol. 2008, 54, 1164-1175.

54. Slekovec, C.; Leroy, J.; Huttner, A.; Ruyer, O.; Talon, D.; Hocquet, D.; Bertrand, X. When the precautionary principle disrupts 3 years of antibiotic stewardship: Nitrofurantoin in the treatment of urinary tract infections. J. Antimicrob. Chemother. 2014, 69, 282-284. 


\title{
Chapter 12
}

\section{International Conference "Urogenital Infections and Tuberculosis" in Novosibirsk, Russia, Has Opened New Perspectives in the Fight against Tuberculosis}

\author{
Ekaterina Kulchavenya, Irina Felker and Elena Brizhatyuk
}

\begin{abstract}
The first International Conference "Urogenital Infections and Tuberculosis" was held in Novosibirsk 24-26 October 2013. Three hundred and twelve delegates from 73 cities in 16 countries took part in the conference. Actual problems of urogenital tract infection (UTI) including tuberculosis (TB) as a specific infection were discussed, including: nosocomial infections in urology, various aspects of prostate biopsy, epidemiology and diagnosis of urogenital tuberculosis, gender and age related characteristics of urinary tract infections, and male infertility, etc.
\end{abstract}

Reprinted from Antibiotics. Cite as: Kulchavenya, E.; Felker, I.; Brizhatyuk, E. International Conference "Urogenital Infections and Tuberculosis" in Novosibirsk, Russia, Has Opened New Perspectives in the Fight against Tuberculosis. Antibiotics 2014, 3, 121-127.

\section{Introduction}

The meeting of the ESIU/EAU board at the annual EAU Congress in Paris in 2013 had decided to organize the Conference on Urogenital Tuberculosis (UGTB) in a region with a high prevalence of this disease. As the Novosibirsk Research TB Institute and the TB Department of Novosibirsk Medical University are well-known for their studies in this field, and Siberia is an epidemic region of TB, Novosibirsk was chosen as a place for this Conference. The meeting was organized as a joint event between several medical associations: The European Section of Infection in Urology (ESIU)/European Association of Urology (EAU), International Commission on UTI of the International Society of Chemotherapy for Infection and Cancer (ISC), Russian Society of Urology, Russian National Association of Phthysiologists, Asian Association of Urogenital Tract Infection and Sexually Transmitted Diseases (UTI\&STI), Novosibirsk Research Institute for Tuberculosis and Novosibirsk Medical University, Russia. The Organizing Committee was chaired by Professor Truls E. Bjerklund Johansen, Chair of the ESIU, Professor Kurt G. Naber, Past-President of the ISC, and Honorary Chair of the ISC WG UTI, and Professor Ekaterina Kulchavenya, as President of the Conference. The International Conference "Urogenital Infections and Tuberculosis" was held in Novosibirsk, from 24 to 26 October 2013. Three hundred and twelve delegates from 73 cities in 16 countries (Russia, Germany, Switzerland, Sweden, Israel, South Korea, China, Turkey, Ukraine, Belarus, Tajikistan and others) took part in the conference, and 191 doctors attended via on-line translation. 
One of the conference highlights included the World Health Organization (WHO) report which indicated that 8.6 million people suffer from TB in 2012, including 1.1 million cases among people with HIV. In 2012, 1.3 million people died from TB. In 2012, an estimated 450,000 people developed multidrug-resistant TB (MDR-TB) globally and there were an estimated 170,000 deaths occurred from MDR-TB.

M. tuberculosis may affect any organ of the human body, and next to the lungs the most common sites of the disease are the urogenital system, bones and joints. Unfortunately, because of delayed diagnosis, urogenital tuberculosis (UGTB) often has a complicated course. UGTB may mimic urogenital tract infection (UTI) with common bacteria, a fact that confuses many doctors who are not familiar with TB.

The official opening ceremony was attended by the Minister of Public Health of the Novosibirsk region, Professor Leonid Shaplygin (Figure 1), and the Director of the Novosibirsk TB Research Institute, Head of the TB Department in Novosibirsk State Medical University, Professor Vladimir Krasnov.

Figure1. Minister of Public Health of the Novosibirsk region, Professor Leonid Shaplygin.

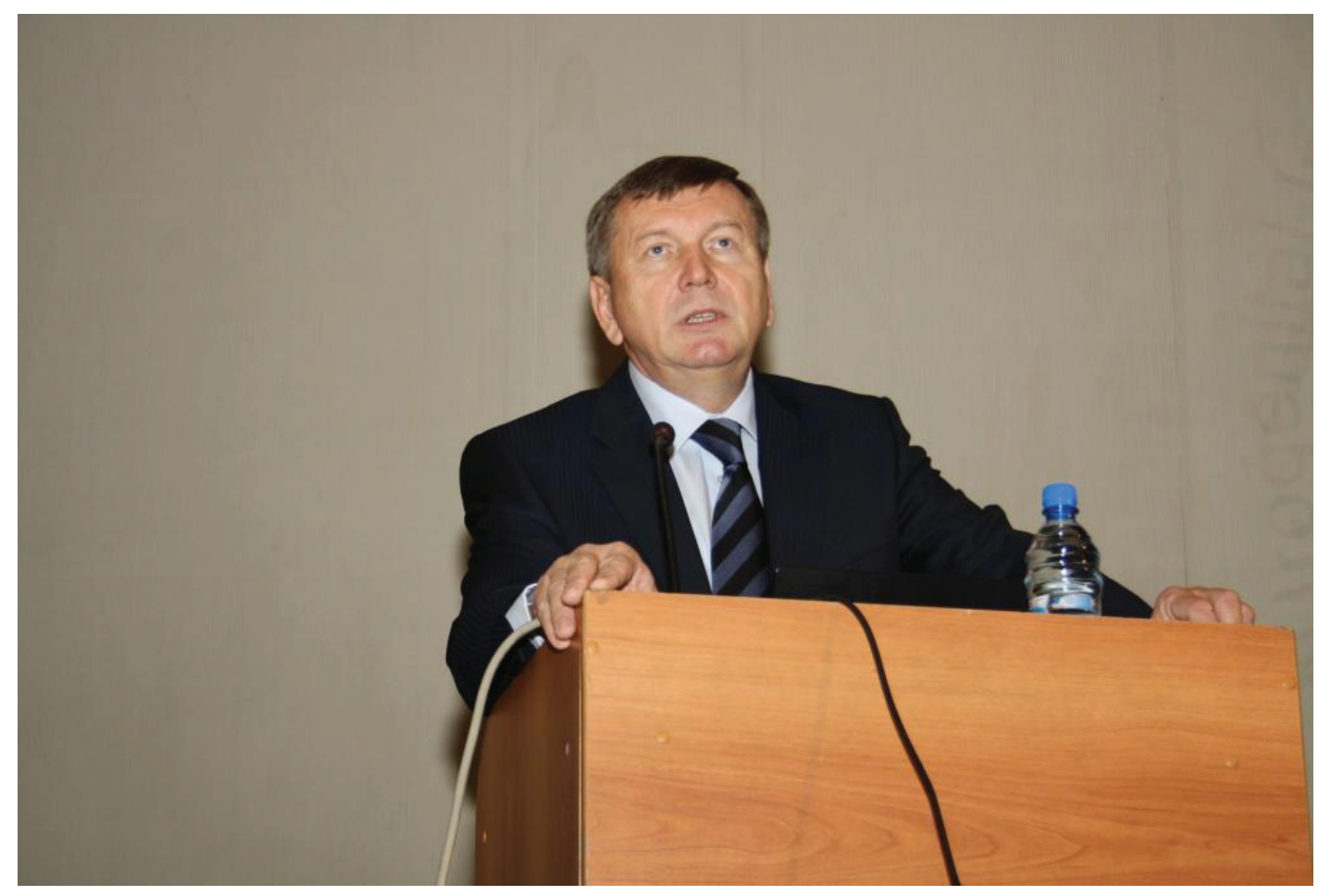

For three days there were parallel lectures, debates and discussions in two halls - in English and Russian languages with simultaneous translation. The following problems were discussed: latent urogenital infections, their bacteriological diagnosis and therapy; complicated urogenital infections, their prevention and treatment; sexually transmitted infections; infectious diseases of the reproductive system as a cause of infertility and sexual dysfunction; interrelation of infections and tumors of the urogenital system: mistakes in diagnosis and features of combined therapy; standards of examination and treatment of patients with urogenital infections; epidemiology, organization of early detection and follow up of patients with urogenital tuberculosis; classification of urogenital tuberculosis; 
diagnosis, therapy and surgery of urogenital tuberculosis; multi-organ tuberculosis, and multi-drug resistance (MDR) with extrapulmonary tuberculosis.

On the first day of the conference, participants were able to attend sections dedicated to the following topics: bladder leukoplakia, kidney stones, and the problem of non-specific cystitis, chronic prostatitis, and sexually transmitted infections. In the session on bladder leukoplakia, the etiology and pathogenesis of this disease were considered in addition to the role of sexually transmitted diseases. Also, the possibilities of minimally invasive procedures as well as the prospects for recovery of anatomy and physiology of the urothelium were demonstrated. A new method of less invasive intravesical laser therapy for bladder leukoplakia was presented (Ekaterina Kulchavenya, Novosibirsk, Russia) with evidence of its superiority compared to transurethral resection. Problems of urolithiasis are by no means new to urology, but they are still quite relevant. The latest techniques and the "gold standard" lithotripsy and litholytic therapy were presented and prevention of infectious complications and preoperative preparation were thoroughly discussed (Fedor Kapsargin, Igor Feofilov, Valentin Isaenko-all from Siberia, Russia). Although the topic of non-specific cystitis in urological practice has been repeatedly considered at various congresses and conferences, the contributions and discussions in this section were again very lively.

On the second day of the Congress, the followings topics were discussed: current issues of nosocomial infections in urology, various aspects of prostate biopsy, epidemiology and diagnosis of urogenital tuberculosis, gender and age related characteristics of urinary tract infections, and male infertility. Possibilities of minimally invasive and endoscopic procedures in urology, as well as modern methods of drainage of the urinary tract and wounds were demonstrated to the audience.

\section{Highlights of the Conference}

Ekaterina Kulchavenya (Novosibirsk, Russia) emphasized in her presentation on the classification of UGTB that UGTB remains an important problem, especially in developing countries, because it is often an overlooked disease. Classification includes exact description of forms and stages of the UGTB, because each stage implies a different approach of management. Thus, accurate classification is the basis for good therapeutic results.

Kidney tuberculosis (KTB) at stage 1-2 should be treated with chemotherapy, KTB at stage 3 requires partial nephrectomy, and KTB at stage 4 is indicated for nephrectomy. A stricture of ureter needs reconstructive surgery in KTB 1-3, but nephron-ureterectomy in KTB-4. Male genital tuberculosis (MGTB) should be treated with chemotherapy; fistulas are treated by surgery. Generalized UGTB, combining both kidney TB and male genital TB should be managed depending on the forms and stages of the kidney and male genital TB. UGTB is a tremendously diverse disease. A unified standard approach is therefore impossible. The common term "UGTB" gives insufficient information about therapy needed, e.g., surgery, and prognosis — and makes evaluation of general epidemiology difficult. In contrast, a more detailed clinical classification will also improve the therapy of UGTB. 
Mete Cek from Trakya University (Turkey) reported the results of a 10-year, worldwide study on the prevalence, structure and characteristics of nosocomial infections in hospitalized urological patients. Besides prevalence and contemporary classifications, also a detailed analysis was presented concerning causes of nosocomial infections, antibiotic resistance patterns of uropathogens, and international practices of antibacterial therapy.

Florian Wagenlehner from the University of Giessen (Giessen, Germany) had two presentations, one on complications of prostate biopsy and the other on new antibiotics in urology. Every year in Europe more than 1 million prostate biopsies are performed, and the risk of infectious complications after this procedure is rising. The author proposed a strategy to minimize the risk of infectious complications and justified the need of targeted antibiotic prophylaxis. In his second presentation he analyzed the global bacterial antibiotic resistance in urology in relation to the antibacterial activity of different classes of existing and new antibiotics in the pipeline. Gernot Bonkat from the University of Basle (Switzerland) presented the isothermal micocalorimetry as a new method for identification of Mycobacterium tuberculosis and drug susceptibility testing. According to the author's data, isothermal microcalorimetry could displace all other culturing methods, since the minimum detection time is only $25 \mathrm{~h}$ and the test easily identifies the drug sensitivity of the pathogen. With regards to UGTB, with this technique the growth rate and doubling time of 4 different mycobacteria could be determined in urine simultaneously.

Topics of the third day of the conference were prevention of urogenital infections, chemotherapy and surgery of urogenital tuberculosis.

Magnus Grabe (Malmö, Sweden) discussed the prevention of infectious complications in urological surgery. He encouraged all participants to follow the recent recommendations of the EAU Guidelines.

Björn Wullt (Lund, Sweden) reviewed in his presentation the optimal management of uncomplicated UTI according to the 2013 updated EAU guidelines. Seung-Ju Lee (Suwon, Korea) described the methods of reconstructive surgery in UGTB. Denis Kholtobin (Novosibirsk, Russia) presented his rather huge own surgical experience. He emphasized the necessity of simultaneous extirpation of bladder and prostate in male patients with bladder TB.

Anna Mordyk (Omsk, Russia) paid special attention to difficulties of bacteriological confirmation of UGTB. The main reasons are: (i) rare and scant mycobacteriuria and (ii) non-optimal previous antibacterial therapy for "urogenital tract infections", which in fact means overlooked UGTB. Olga Alhovik (Novosibirsk, Russia) presented new techniques for rapid identification of M. tuberculosis with BACTEC 960 and GeneXpert. According to data of the Novosibirsk Research TB Institute, in $12.5 \%$ of patients with prostate tuberculosis that initially showed negative culture results, M. tuberculosis was finally found with these new methods.

On Wellcome Reception after first day of the Conference some participants who made biggest contribution in this event were awarded with Diplomas and Prizes, Figure 2 shows the moment of awarding Professor Florian Wagenlehner (Germany). 
Figure 2. President of the Conference Professor Ekaterina Kulchavenya presents an award to Professor Florian Wagenlehner.

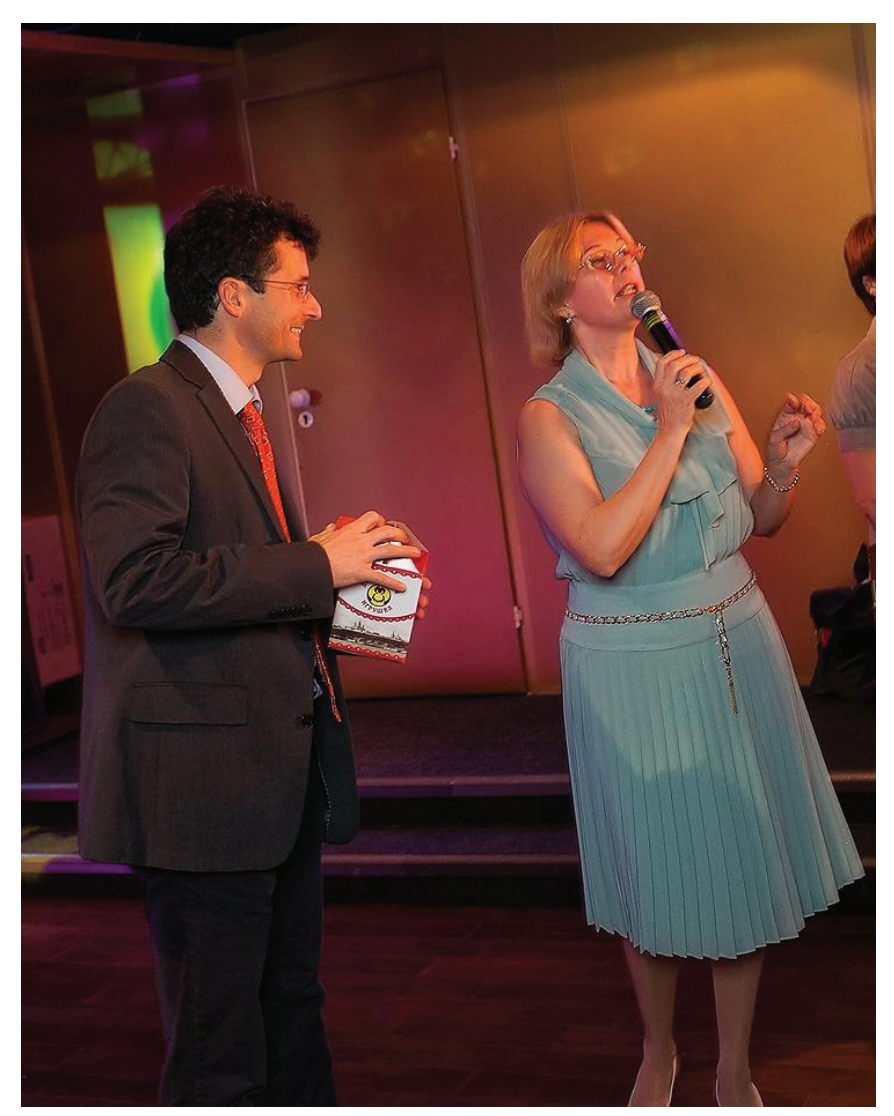

\section{Conclusions}

We do not know the real incidence and prevalence of UGTB as it includes KTB, MGTB, and female genital TB. All these forms of TB have their own clinical features and require their own approaches to the diagnosis and management. Before anti-TB drugs were created, the prevalence of UGTB was huge. Every fifth urological in-patient had UGTB, more than third of all pyonephrosis were due to TB [1]. Nowadays in Siberia, about 300 patients with urological tuberculosis are revealed annually [2]. In developed countries, from $2 \%$ to $10 \%$ patients with PTB have also UGTB; in developing countries the proportion increases up to $15 \%-20 \%$ [3]. In Europe, UGTB is diagnosed more often in migrants, than in inhabitants [4,5]. About $20 \%$ of patients cured from PTB, had EPTB later, mostly UGTB [6].

This first conference brought together urologists and TB specialists not only from the Siberian region, but also from the whole of Russia and other countries as well. The outstanding quality of the presentations and the lively discussions during the congress underlined the high degree of relevance of this event. Participants were able not only to gain useful information, but also to find new partners for further joint research.

There are many un-solved problems in TB urology. Unique terminology, classification, and approach to diagnosis, therapy and surgery for patients UGTB are needed. Heated discussions, hot debates and arguments between participants at this Conference resulted in agreement on key points. 
The take home messages emphasized the need for more awareness regarding UGTB in many regions of the world, considering that UGTB is a sexually transmitted disease, as M. tuberculosis can be found in the ejaculate of half of the TB patients [7]. A better classification of UGTB is also needed to improve treatment recommendations and report the outcome of surgical interventions, particularly for TB of the bladder and prostate.

After Conference memory picture of the faculty was made in the hall of the Congress Center (Figure 3).

Figure 3. Conference faculty in the conference hall in Novosibirsk.

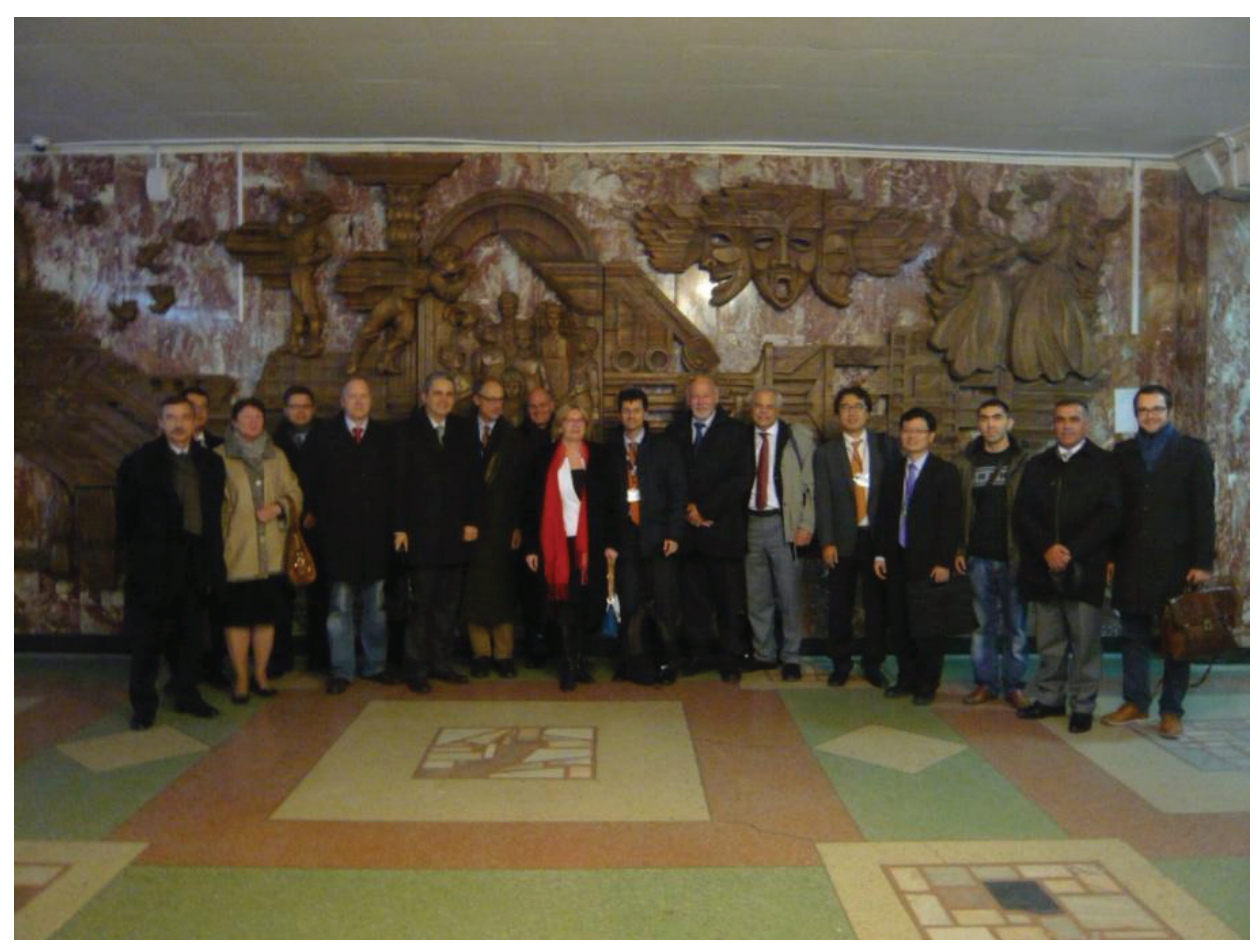

\section{Acknowledgment}

Authors would like to thank Vladimir Krasnov, Tatyana Petrenko, Victor Khomyakov, Denis Kholtobin, Alexander Osadchiy, Victoriya Dement'eva, Sventlana Mal'kova for their assistance in the organization this Conference.

\section{Author Contributions}

Ekaterina Kulchavenya - preparing the text, translation, edition; Irina Felker-preparing the text, translation; Elena Brizhatyuk - preparing discussion, literature review.

\section{Conflicts of Interest}

We declare potential conflict of Interest as the authors are organizer and participant of the conference "Urogenital Infections and Tuberculosis". 


\section{References}

1. Marion, G. Traite d'Urologie; Masson et Cie: Paris, France, 1940; pp. 34-42.

2. Kulchavenya, E. Best practice in the diagnosis and management of Urogenital Tuberculosis. 2013, 5, 143-151.

3. Figueiredo, A.A.; Lucon, A.M. Urogenital tuberculosis: Update and review of 8961 cases from the world literature. Rev. Urol. 2008, 10, 207-217.

4. Lenk, S. Genitourinary tuberculosis in Germany: Diagnosis and treatment. Der Urologe. Ausg. A 2011, 50, 1619-1627.

5. Singh, D.D.; Vogel, M.; Müller-Stöver, I.; El Scheich, T.; Winzer, M.; Göbels, S.; Hüttig, F.; Heinrich, S.; Mackenzie, C.; Jensen, B.; et al. TB or not TB? Difficulties in the diagnosis of tuberculosis in HIV-negative immigrants to Germany. Eur. J. Med. Res. 2011, 16, 381-384.

6. Lenk, S.; Schroeder, J. Genitourinary tuberculosis. Curr. Opin. Urol. 2001, 11, 93-98.

7. Aphonin, A.B.; Perezmanas, E.O.; Toporkova, E.E.; Khodakovsky, E.P. Tuberculous infection as sexually transmitted infection (in Russian). Vestn. Poslediplom. Obraz. 2006, 3-4, 69-71. 
MDPI AG

Klybeckstrasse 64

4057 Basel, Switzerland

Tel. +41616837734

Fax +41613028918

http://www.mdpi.com/

Antibiotics Editorial Office

E-mail: antibiotics@mdpi.com http://www.mdpi.com/journal/antibiotics 
Giva MDPI - Basel - Beijing • Wuhan

74 ISBN 978-3-906980-80-5

回证! www.mdpi.com 